\title{
Off-pump coronary artery bypass grafting improves short-term outcomes in high-risk patients compared with on-pump coronary artery bypass grafting: Meta-analysis
}

\author{
Mariusz Kowalewski, MD, ${ }^{\mathrm{a}, \mathrm{b}, \mathrm{c}}$ Wojciech Pawliszak, MD, ${ }^{\mathrm{a}}$ Pietro Giorgio Malvindi, MD, ${ }^{\mathrm{d}}$ \\ Marek Pawel Bokszanski, MD, ${ }^{\mathrm{a}}$ Damian Perlinski, MD, ${ }^{\mathrm{a}}$ Giuseppe Maria Raffa, MD, \\ Magdalena Ewa Kowalkowska, MD, ${ }^{\mathrm{c}, \mathrm{f}}$ Katarzyna Zaborowska, RN, ${ }^{\mathrm{a}}$ Eliano Pio Navarese, MD, PhD, ${ }^{\mathrm{c}, \mathrm{g}}$ \\ Michalina Kolodziejczak, MD, ${ }^{\mathrm{c}, \mathrm{h}}$ Janusz Kowalewski, MD, PhD, ${ }^{\mathrm{i}}$ Giuseppe Tarelli, MD, ${ }^{\mathrm{j}}$ \\ David Paul Taggart, MD, PhD, ${ }^{\mathrm{k}}$ and Lech Anisimowicz, MD, $\mathrm{PhD}^{\mathrm{a}}$
}

\begin{abstract}
Objectives: To assess the benefits and risks of off-pump coronary artery bypass $(\mathrm{OPCAB})$ versus coronary artery bypass grafting $(\mathrm{CABG})$ through a meta-analysis of randomized controlled trials (RCTs), and to investigate the relationship between outcomes and patient risk profile.

Methods: PubMed, Embase, the Cumulative Index of Nursing and Allied Health Literature, Scopus, Web of Science, Cochrane Library, and major conference proceedings databases were searched for RCTs comparing OPCAB and CABG and reporting short-term $(\leq 30$ days $)$ outcomes. Endpoints assessed were all-cause mortality, myocardial infarction (MI), and cerebral stroke.
\end{abstract}

Results: The meta-analysis included 100 studies, with a total of 19,192 subjects. There was no difference between the 2 techniques with respect to all-cause mortality and MI (odds ratio [OR], 0.88; 95\% confidence interval [CI], $0.71-1.09 ; P=.25 ; I^{2}=0 \%$ and OR, $0.90 ; 95 \% \mathrm{CI}, 0.77-1.05 ; P=.19$; $I^{2}=0 \%$, respectively). OPCAB was associated with a significant $28 \%$ reduction in the odds of cerebral stroke (OR, 0.72; 95\% CI, 0.56-0.92; $P=.009 ; I^{2}=0 \%$ ). A significant relationship between patient risk profile and benefits from OPCAB was found in terms of all-cause mortality $(P<.01)$, MI $(P<.01)$, and cerebral stroke $(P<.01)$.

Conclusions: OPCAB is associated with a significant reduction in the odds of cerebral stroke compared with conventional CABG. In addition, benefits of $\mathrm{OPCAB}$ in terms of death, MI, and cerebral stroke are significantly related to patient risk profile, suggesting that OPCAB should be strongly considered in high-risk patients. (J Thorac Cardiovasc Surg 2016;151:60-77)

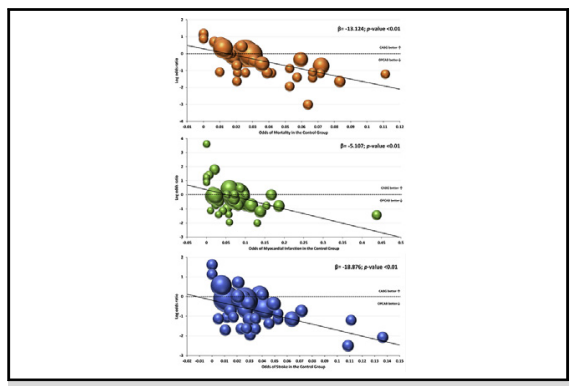

Risk profile meta-regression. The benefits of OPCAB are greater in high- risk patients.

\section{Central Message}

Benefits of OPCAB in terms of death, MI, and cerebral stroke are significantly related to patient risk profile when compared with CABG.

\section{Perspective}

CABG remains the gold standard for surgical coronary revascularization; however, the present meta-analysis clearly demonstrated significantly reduced odds of cerebral stroke with OPCAB. More importantly, benefits of OPCAB in terms of death, MI, and cerebral stroke are significantly related to patient risk profile, suggesting that OPCAB should be strongly considered in high-risk patients.

See Editorial Commentary page 78.

See Editorial page 4.

\footnotetext{
From the ${ }^{a}$ Department of Cardiac Surgery, Dr Antoni Jurasz Memorial University Hospital, Bydgoszcz, Poland; ${ }^{\mathrm{b}}$ Faculty of Health Sciences, Nicolaus Copernicus University, Collegium Medicum, Bydgoszcz, Poland; 'Systematic Investigation and Research on Interventions and Outcomes (SIRIO) MEDICINE Research Network, Düsseldorf, Germany; ${ }^{\mathrm{d}}$ University Hospital Southampton NHS Foundation Trust, Wessex Cardiothoracic Centre, Southampton, United Kingdom; ${ }^{\mathrm{e}}$ Department for the Treatment and Study of Cardiothoracic Diseases and Cardiothoracic Transplantation, IRCCS - ISMETT (Istituto Mediterraneo per i Trapianti e Terapie ad alta specializzazione), Palermo, Italy; ${ }^{\mathrm{f}}$ Department and Clinic of Obstetrics, Gynecology, and Oncological Gynecology, Collegium Medicum, Bydgoszcz, Poland; ${ }^{\mathrm{g}}$ Department of Internal Medicine, Division of Cardiology, Pulmonology and Vascular Medicine, Heinrich-Heine-University, Düsseldorf, Germany; ${ }^{\mathrm{h}}$ Collegium Medicum in Bydgoszcz, Nicolaus Copernicus University, Toruń, Poland; ${ }^{\mathrm{i}}$ Lung Cancer and Thoracic Surgery Department, Collegium Med-
}

icum in Bydgoszcz, Nicolaus Copernicus University, Torun, Poland; ${ }^{\mathrm{j}}$ Department of Cardiac Surgery, Humanitas Clinical and Research Center, Rozzano, Milan, Italy; and ${ }^{\mathrm{k}}$ Department of Cardiac Surgery, John Radcliffe Hospital, Oxford, United Kingdom.

M.K. and W.P. contributed equally to the present work.

Received for publication May 1, 2015; revisions received July 27, 2015; accepted for publication Aug 12, 2015; available ahead of print Sept 30, 2015

Address for reprints: Mariusz Kowalewski, MD, Department of Cardiac Surgery,

Dr Antoni Jurasz Memorial University Hospital, Maria Curie Sklodowska Str 9,

85-094 Bydgoszcz, Poland (E-mail: kowalewskimariusz@gazeta.pl). $0022-5223 / \$ 36.00$

Copyright (C) 2016 by The American Association for Thoracic Surgery http://dx.doi.org/10.1016/j.jtcvs.2015.08.042 


\begin{tabular}{|c|c|}
\hline \multicolumn{2}{|c|}{ Abbreviations and Acronyms } \\
\hline CABG & $=$ Coronary artery bypass grafting \\
\hline CI & $=$ Confidence interval \\
\hline CAD & $=$ Coronary artery disease \\
\hline CORONARY & $Y=$ CABG Off or On Pump \\
\hline & Revascularization Study \\
\hline ECC & $=$ Extracorporeal circulation \\
\hline EuroSCORE & $\begin{aligned}= & \text { European System for Cardiac } \\
& \text { Operative Risk Evaluation }\end{aligned}$ \\
\hline MI & $=$ Myocardial infarction \\
\hline NNT & $=$ Number needed to treat \\
\hline OPCAB & $=$ Off-pump coronary artery bypass \\
\hline OR & $=$ Odds ratio \\
\hline PCI & $=$ Percutaneous coronary intervention \\
\hline PRISMA & $\begin{array}{l}=\text { Preferred Reporting Items for } \\
\text { Systematic Reviews and Meta- } \\
\text { Analyses }\end{array}$ \\
\hline RCT & $=$ Randomized controlled trial \\
\hline ROOBY & $\begin{aligned}= & \text { Veterans Affairs Randomized On/ } \\
& \text { Off Bypass Trial }\end{aligned}$ \\
\hline
\end{tabular}

Supplemental material is available online.

Coronary artery bypass grafting $(\mathrm{CABG})$ is associated with reduction of mortality and remains a standard of care in patients with extensive coronary artery disease (CAD) as compared to percutaneous coronary intervention (PCI) and medical treatment alone. ${ }^{1-3}$ CABG with the use of cardiopulmonary bypass $(\mathrm{CPB})$ is recognized as the gold standard technique in terms of safety and effectiveness for surgical myocardial revascularization. A further effort in minimizing the occurrence of some complications related to conventional $\mathrm{CABG}$ has led to the development of off-pump (OPCAB) technique in which the anastomoses are performed on the beating heart. ${ }^{4}$ Observational studies have suggested that, by avoiding the negative effects of $\mathrm{CBP}, \mathrm{OPCAB}$ may substantially reduce the rate of mortality and morbidity when compared with conventional $\mathrm{CABG} .^{5-7}$ These benefits, however, have never been confirmed in a single randomized controlled trial (RCT). First large Veterans Affairs Randomized On/Off Bypass (ROOBY) trial, ${ }^{8}$ found no significant difference between off-pump and on-pump CABG in the rate of the 30-day composite endpoint of death, reoperation, new mechanical support, cardiac arrest, coma, stroke, or renal failure $(7.0 \%$ and $5.6 \%$, respectively; $P=.19$ ). Similarly, the largest experience to date, the CABG Off or On Pump Revascularization Study (CORONARY), ${ }^{9}$ which randomized more than 4700 patients to OPCAB and conventional $\mathrm{CABG}$, showed no significant difference between these 2 techniques in terms of 30-day rate of mortality, myocardial infarction (MI), stroke, or renal failure requiring dialysis. Although various analyses have suggested a beneficial effect of the OPCAB technique in selected subsets of high-risk and elderly patients, ${ }^{10,11}$ the evidence is not sufficiently powerful to allow for definite conclusions. Thus, the aim of the present study was to conduct an updated meta-analysis of RCTs of OPCAB versus CABG and to evaluate, by meta-regression analysis, whether the results may be related to patient risk profile.

\section{METHODS}

\section{Data Sources and Search Strategy}

Established methods were used in compliance with the Preferred Reporting Items for Systematic Reviews and Meta-Analyses (PRISMA) in health care interventions statement. ${ }^{12}$ A PRISMA checklist is available as Appendix Table 1. We conducted a search for relevant RCTs comparing OPCAB with conventional CABG until March 2015 through PubMed (Appendix Table 2), Embase, the Cumulative Index of Nursing and Allied Health Literature, the Web of Science, the Cochrane Register of Controlled Clinical Trials, and Google Scholar, as well as published proceedings from major cardiac, thoracic and cardiothoracic, and cardiology society meetings. Abstracts were eligible for detailed assessment if available online and reporting outcomes of interest. Search terms included off-pump, on-pump, with/without cardiopulmonary bypass, OPCAB, CABG, extracorporeal circulation (ECC), conventional, beating heart, randomized trial, and trial. No language, publication date, or publication status restriction was imposed. Both blinded and open-label trials were considered eligible. The most updated or inclusive data for each study were used for abstraction. References of original and review articles were cross-checked.

\section{Selection Criteria and Quality Assessment}

Citations were screened at the title/abstract level and retrieved as full reports if they fulfilled the inclusion criteria: human study and RCT comparing first-time $\mathrm{OPCAB}$ and $\mathrm{CABG}$ irrespective of access to the thorax (full or partial median sternotomy/thoracotomy). The exclusion criteria were a prospective cohort and quasi-randomized study, a study with a concomitant surgical procedure other than $\mathrm{CABG}$, a study with a particular medical or invasive treatment in 1 arm (eg, PCI + OPCAB vs CABG), CABG with an on-pump beating heart, miniaturized ECC used in the $\mathrm{CABG}$ arm, robot-assisted $\mathrm{CABG}$, and pediatric cardiac surgery.

Two independent reviewers (M.K. and D.P.) selected the studies for inclusion and extracted studies and patient characteristics of interest and relevant outcomes; divergences were resolved by consensus after discussion with a third reviewer (L.A.). Two authors (K.Z. and M.E.K.) independently assessed the trials' eligibility and risk of bias. The risk of bias for randomized studies was assessed using the components recommended by the Cochrane Collaboration, ${ }^{13}$ including random sequence generation and random allocation; allocation concealment; blinding of participants, personnel, and outcome assessors; incomplete outcome data; selective outcome reporting; and other sources of bias. Any discrepancies in bias assessment between the assessors were recorded and reported as Cohen's $\kappa .{ }^{14}$

\section{Outcome Measure}

The endpoints assessed were all-cause mortality, MI, and cerebral stroke occurring within 30 days after the surgical procedure. Data were extracted in duplicate by 2 investigators (M.K. and D.P.) and independently 


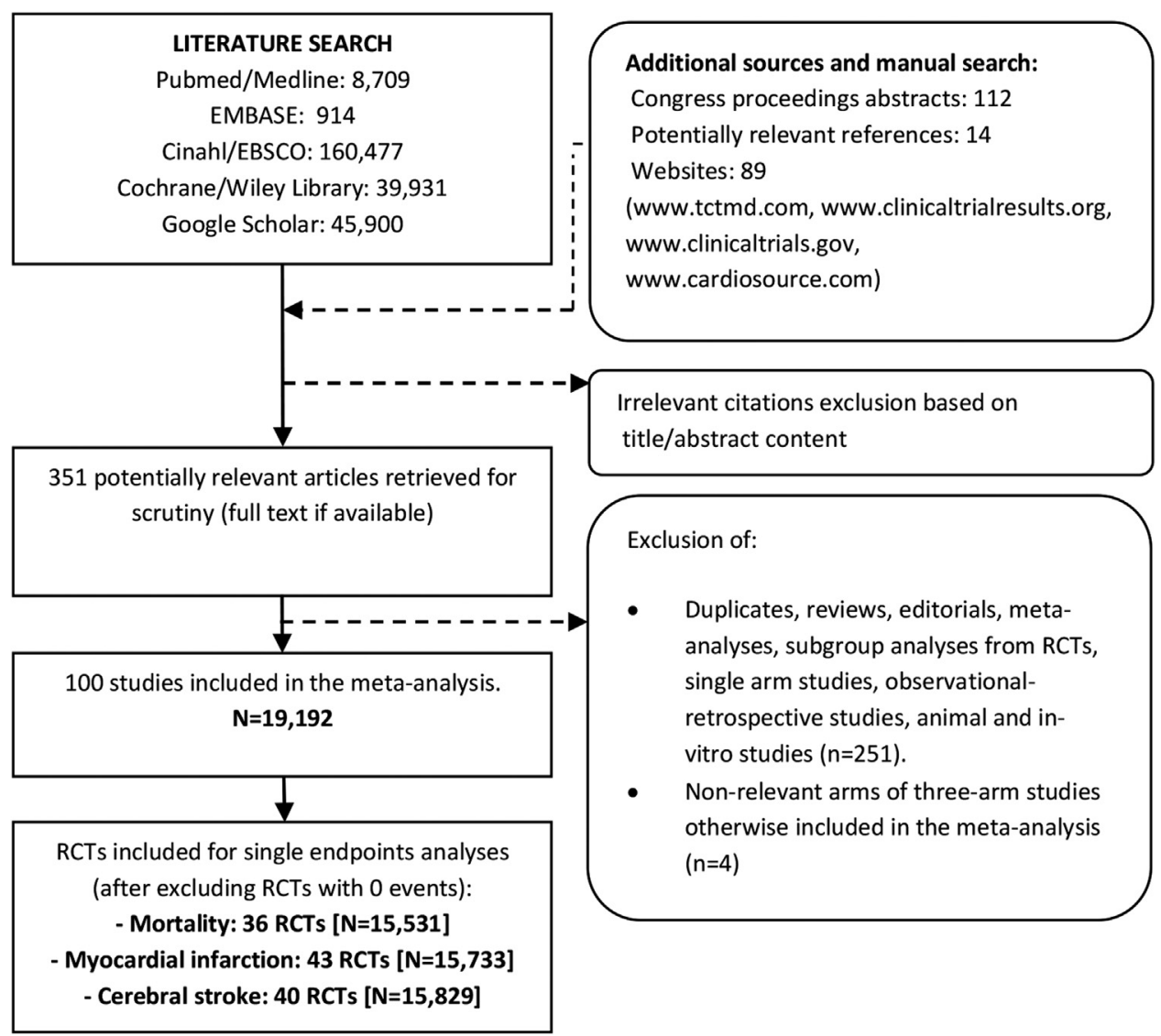

FIGURE 1. Flow diagram of the review process according to the PRISMA statement. RCT, Randomized controlled trials.

verified by a third investigator (L.A.). Disagreements were resolved by consensus.

\section{Statistical Analysis}

Data were analyzed according to the intention-to-treat principle. Odds ratios (ORs) and $95 \%$ confidence intervals (CIs) were used as summary statistics. Heterogeneity was assessed using Cochran's $Q$ test. ${ }^{15}$ To overcome the low statistical power of Cochran's $Q$ test, the statistical inconsistency test $I^{2}=[(Q-\mathrm{df}) / Q] \times 100 \%$, where $Q$ is the $\chi^{2}$ statistic and $\mathrm{df}$ is its degrees of freedom, was used to assess heterogeneity. This determines the percentage of interstudy variation, with values ranging from $0 \%$ to $100 \%$. An $I^{2}$ value of $<40 \%$ indicated no obvious heterogeneity, values between $40 \%$ and $70 \%$ suggested moderate heterogeneity, and values $>70 \%$ were considered to represent high heterogeneity.

We calculated pooled ORs using the Mantel-Haenszel random-effects model, with weight assigned to each included study adjusted to include a measure of variation $\left(\tau^{2}\right)$ in the effects reported between studies. This approach estimates the amount of between-study variation by comparing each study's result with a Mantel-Haenszel fixed-effect meta-analysis result, and is most conservative in case of low between-study heterogeneity. ${ }^{16}$ In this model, trials with zero events in both groups were excluded from the pooled analysis, because adding a correction factor of 0.5 to both zero event groups to force these trials into the analysis might have biased the results toward the null hypothesis, owing to the increased number of pseudoevents. ${ }^{17}$

In a prespecified sensitivity analysis, we recalculated the results in random-effects models with an inverse variance (DerSimonian-Laird) method, with natural weighting, ${ }^{18}$ in Hartung-Knapp-Sidik Jonkman ${ }^{19}$ and Mantel-Haenszel fixed-effect models, and using the Peto method, ${ }^{20,21}$ the preferred approach when intervention effects are small (ie, ORs are close to 1) and events are not particularly common. In addition, we accounted for studies that reported zero events for both investigated arms in a collapsed $4 \times 4$ table. To further corroborate the obtained results, we performed analyses for all clinical outcomes expressed as standardized difference in means and after arcsine transformation of risk difference. ${ }^{22}$ We calculated the number needed to treat (NNT) and number needed to harm as well.

We evaluated potential publication bias for the primary endpoint by constructing a "funnel plot" in which the standard error of the $\log$ OR was plotted against the OR. The asymmetry of the plot was estimated both visually and by a linear regression approach. ${ }^{23}$ A weighted random-effects risk profile meta-regression analyses for all investigated outcomes were performed by regressing log OR against the odds in the control group, using the inverse of the variance of the log odds as weight. ${ }^{24}$ Finally, we addressed the influence of each study and potential publication bias by testing whether deleting each study in turn would have resulted in a significant change in the pooled results of the meta-analysis for all endpoints assessed. We checked whether our results were driven by small-sample studies, by deleting studies enrolling $<50$ and $<100$ participants and repeating the calculations. Review Manager 5.1 (Nordic Cochrane Center, Købehvn, Denmark), Comprehensive Meta-Analysis version 2 (Biostat; Englewood, NJ) and R version 3.2.1 (R Foundation for Statistical Computing, Vienna, Austria; http://www.R-project.org/) with meta package "meta" (http://CRAN.R-project.org/package/meta), were used for statistical computations. Results of the weighted 
random-effects risk profile meta-regression analyses are reported as $\beta$ coefficients and 2 -sided $P$ values.

\section{RESULTS}

A PRISMA flow chart, describing the process of publication screening and the reasons for exclusion, is shown in Figure 1. Among the 351 potentially relevant reports retrieved for scrutiny, 251 were excluded during secondary screening. The remaining 100 studies, which included a total of 19,192 participants, were eventually included for data abstraction and entered the analysis. All published studies were retrieved as full texts. The characteristics of the included studies are listed in Table 1. Patients were evenly randomized to the OPCAB group $(\mathrm{n}=9574)$ and the CABG group $(\mathrm{n}=9618)$. The estimated mean age was 67.2 years overall, and was slightly higher in the OPCAB group compared with the CABG group (67.4 vs 66.9 years). Internal mammary artery use exceeded $96.3 \%$. Fewer distal anastomoses were performed in the OPCAB group compared with the $\mathrm{CABG}$ group (mean, 2.91 vs 3.10; weighted mean difference, -0.19 ; $95 \% \mathrm{CI},-0.25$ to $\left.-0.14 ; P<.01 ; I^{2}=43 \%\right)$. The rate of conversion from OPCAB to CABG rates was reported in 81 studies and ranged from $0 \%$ to $22.5 \%$. The proportion of patients undergoing "no-touch" anaortic OPCAB was seldom reported (14 studies); the rate of proximal anastomosis device use was reported in only 1 study. Detailed patient baseline characteristics are presented in Appendix Table 3. The risk of bias of the included studies is characterized in Appendix Table 4. Endpoint definitions are provided in Appendix Table 5.

\section{Mortality}

Thirty-six studies, with a total of 15,531 subjects, contributed to the analysis of $\leq 30$-day all-cause mortality. No signs of publication bias were demonstrated by visual inspection of the funnel plot (Appendix Figure 1). The mortality rate was $2.25 \%$ overall and ranged from $2.04 \%$ (159 of 7779 ) in the OPCAB group to $2.45 \%$ (190 of 7752) in the CABG group. No difference was evident between the 2 techniques (OR, $0.88 ; 95 \% \mathrm{CI}$, $0.71-1.09 ; P=.25 ; I^{2}=0 \%$ ) (Figure $2, A$ ). A significant linear relationship was observed between $\log \mathrm{OR}$ of all-cause mortality weighted against the odds of mortality $(\beta=-13.124 ; P<.01$; Figure $2, B)$ in the control group. The NNT was 246.

\section{Myocardial Infarction}

MIs were observed in 43 trials with a total of 15,733 subjects, with an overall incidence of $4.49 \%$. Analysis of a funnel plot did not reveal any signs of publication bias (Appendix Figure 2). No differences were found between OPCAB and CABG with respect to MI (OR, $0.90 ; 95 \%$ CI, $0.77-1.05 ; P=.19 ; I^{2}=0 \%$ ); the event rate was $4.31 \%$ (340 of 7881 ) for OPCAB and $4.67 \%$ (367 of 7852) for CABG (Figure 3, A). A significant linear relationship was observed between log OR of MI weighted against the odds of MI $(\beta=-5.107 ; P<.01)$ in the control group (Figure 3, $B$ ). The NNT was 278 .

\section{Cerebral Stroke}

The incidence of stroke was reported in 40 RCTs, with a total of 15,829 participants, with an overall rate of $1.67 \%$. No signs of publication bias were observed in a funnel plot (Appendix Figure 3). OPCAB was associated with a significant $(28 \%)$ reduction in the odds of stroke compared with CABG (OR, 0.72; 95\% CI, 0.56-0.92; P = .009; $I^{2}=0 \%$ ); event rates ranged from $1.34 \%$ (106 of 7925) in the OPCAB group to $2.00 \%$ (158 of 7904$)$ in the CABG group (Figure 4, A). A significant linear relationship between log OR of stroke and the odds of stroke in the control group $(\beta=-18.876 ; P<.01)$ was demonstrated (Figure $4, B$ ). The NNT was 152.

\section{Sensitivity Analyses}

Our results remained consistent after exclusion of studies at high risk of bias. We repeated calculations for all-cause mortality, MI, and stroke using random-effects models with an inverse variance (DerSimonian-Laird) method, by natural weighting, with Hartung-Knapp-Sidik Jonkman and Mantel-Haenszel fixed-effect models, and with the Peto OR method and collapsed $4 \times 4$ table, and found no change in either the direction or the magnitude of the estimates (Figure 5, $A-C$ ). Deleting each study at turn and repeating the calculations did not change the results either. Deleting small-sample studies did not affect the final estimates for all outcomes, either after deleting studies enrolling $<50$ participants (Appendix Figures 4-6) or after deleting studies enrolling $<100$ participants (Appendix Figures 7-9). Analyses performed for all clinical outcomes and expressed as standardized difference in means (Appendix Figures 10-12), and after arcsine transformation of risk differences (Appendix Figures 13-15), remained consistent with primary estimates.

\section{DISCUSSION}

The present meta-analysis of RCTs represents the largest ( $\mathrm{n}=19,192$ participants) comprehensive source of trial-based evidence on the efficacy of OPCAB compared with conventional $\mathrm{CABG}$, focusing on short-term outcomes. By its unique design, this analysis is the first report to investigate the causal relationship between underlying patient risk profile and the benefits of off-pump coronary revascularization. Our main findings can be summarized as follows. First, OPCAB was associated with a significant $(28 \%)$ reduction in the odds of $\leq 30$-day cerebral stroke. Second, in the overall analysis, there was no difference between the 2 techniques with 
TABLE 1. Characteristics of included studies

\begin{tabular}{|c|c|c|c|c|c|c|c|c|}
\hline Study ${ }^{\text {reference }}$ & Year & Primary endpoint & $\begin{array}{l}\text { Randomization } \\
\text { (ITT) }\end{array}$ & $\begin{array}{c}\text { Age, }, y \\
\text { mean } \pm \text { SD }\end{array}$ & Extent of CAD & $\begin{array}{c}\text { IMA use, } \\
\% \%\end{array}$ & $\begin{array}{l}\text { No. of } \\
\text { grafts* }\end{array}$ & $\begin{array}{c}\text { Risk of } \\
\text { bias }\end{array}$ \\
\hline Al-Ruzzeh et al ${ }^{\mathrm{El}}$ & 2006 & $\begin{array}{l}\text { Graft patency at } 3 \mathrm{mo} \text {, } \\
\text { neurocognitive } \\
\text { function at } 6 \mathrm{wk} \text { and } \\
6 \mathrm{mo} \text {, and } \mathrm{HRQoL}\end{array}$ & $\begin{array}{l}\text { OPCAB }(\mathrm{n}=84) \\
\text { CABG }(\mathrm{n}=84)\end{array}$ & $\begin{array}{l}63.1 \pm 11 \\
63.1 \pm 9.6\end{array}$ & $\begin{array}{l}>50 \% \mathrm{MV}-\mathrm{CAD} \\
>50 \% \mathrm{MV}-\mathrm{CAD}\end{array}$ & $\begin{array}{l}48.0 \\
49.1\end{array}$ & $\begin{array}{l}2.73 \\
2.76\end{array}$ & Low \\
\hline \multirow[t]{2}{*}{ Alwan et $\mathrm{al}^{\mathrm{E} 2}$} & \multirow[t]{2}{*}{2004} & \multirow[t]{2}{*}{$\begin{array}{l}\text { Myocardial injury } \\
\text { markers }\end{array}$} & OPCAB $(\mathrm{n}=35)$ & $61 \pm 11$ & $\begin{array}{l}>50 \% \text { MV-CAD; } \\
\text { LM } 17.1 \%\end{array}$ & 100 & $2.3 \pm 1.1$ & \multirow[t]{2}{*}{ Unclear } \\
\hline & & & $\mathrm{CABG}(\mathrm{n}=35)$ & $66 \pm 7$ & $\begin{array}{c}>50 \% \text { MV-CAD } \\
\text { LM } 25.7 \%\end{array}$ & 100 & $2.5 \pm 0.8$ & \\
\hline Ascione et al ${ }^{\mathrm{E} 3}$ & 2005 & $\begin{array}{l}\text { Retinal microvascular } \\
\text { damage }\end{array}$ & $\begin{array}{l}\operatorname{OPCAB}(\mathrm{n}=10) \\
\text { CABG }(\mathrm{n}=10)\end{array}$ & $\begin{array}{l}63.4 \pm 16.4 \\
61.2 \pm 9.8\end{array}$ & $\begin{array}{l}>50 \% \mathrm{MV}-\mathrm{CAD} \\
>50 \% \mathrm{MV}-\mathrm{CAD}\end{array}$ & NR & $\begin{array}{l}2.4 \\
2.5\end{array}$ & Unclear \\
\hline Ascione et $\mathrm{al}^{\mathrm{E} 4}$ & 2006 & $\begin{array}{l}\text { Small intestine } \\
\text { function }\end{array}$ & $\begin{array}{l}\text { OPCAB }(n=20) \\
\text { CABG }(n=20)\end{array}$ & $\begin{array}{l}63.2 \pm 8.8 \\
62.4 \pm 9.7\end{array}$ & $\begin{array}{l}>50 \% \text { MV-CAD } \\
>50 \% \text { MV-CAD }\end{array}$ & NR & $\begin{array}{l}2.5 \\
3.0\end{array}$ & Low \\
\hline Baker et $\mathrm{al}^{\mathrm{E} 5}$ & 2001 & $\begin{array}{l}\text { Neuropsychological } \\
\text { outcomes and } \\
\text { myocardial injury } \\
\text { markers }\end{array}$ & $\begin{array}{l}\text { OPCAB }(n=12) \\
\text { CABG }(n=14)\end{array}$ & $\begin{array}{l}61.7 \pm 11.7 \\
65.9 \pm 8.3\end{array}$ & $\begin{array}{l}>50 \% \text { MV-CAD } \\
>50 \% \text { MV-CAD }\end{array}$ & $\begin{array}{l}83.3 \\
100\end{array}$ & $\begin{array}{l}2.2 \pm 0.6 \\
2.5 \pm 0.5\end{array}$ & Unclear \\
\hline Moller et al $[\mathrm{BBS}]^{\mathrm{E} 6}$ & 2010 & $\begin{array}{l}\text { MACCE (all-cause } \\
\text { mortality, acute } \\
\text { MI, cardiac arrest } \\
\text { with successful } \\
\text { resuscitation, } \\
\text { LCOS/cardiogenic } \\
\text { shock, stroke, and } \\
\text { coronary } \\
\text { reintervention) }\end{array}$ & $\begin{array}{l}\text { OPCAB }(n=177) \\
\text { CABG }(n=164)\end{array}$ & $\begin{array}{l}76.1 \pm 5.2 \\
75.6 \pm 4.9\end{array}$ & $\begin{array}{l}>50 \% \text { MV-CAD } \\
>50 \% \text { MV-CAD }\end{array}$ & $\begin{array}{l}95 \\
93\end{array}$ & $\begin{array}{l}3.22 \pm 0.72 \\
3.34 \pm 0.76\end{array}$ & Low \\
\hline $\begin{array}{l}\text { Angelini et al } \\
\qquad[\text { BHACAS 1] }]^{\mathrm{E} 7, \mathrm{E} 8}\end{array}$ & 2002 & $\begin{array}{l}\text { Short-term morbidity } \\
\text { and use of health } \\
\text { care resources }\end{array}$ & $\begin{array}{l}\text { OPCAB }(n=100) \\
\text { CABG }(n=100)\end{array}$ & $\begin{array}{l}62.2 \pm 9.6 \\
61.7 \pm 8.6\end{array}$ & NR & $\begin{array}{l}93 \\
95\end{array}$ & $\begin{array}{l}2.23 \pm 0.83 \\
2.31 \pm 0.86\end{array}$ & Low \\
\hline $\begin{array}{l}\text { Angelini et al } \\
\qquad \text { [BHACAS 2] }^{\mathrm{E7}}\end{array}$ & 2002 & $\begin{array}{l}\text { Short-term morbidity } \\
\text { and use of health } \\
\text { care resources }\end{array}$ & $\begin{array}{l}\text { OPCAB }(n=100) \\
\text { CABG }(n=101)\end{array}$ & $\begin{array}{l}61.2 \pm 9.2 \\
63.8 \pm 8.5\end{array}$ & NR & $\begin{array}{l}99 \\
98\end{array}$ & $\begin{array}{l}\text { NA } \\
\text { NA }\end{array}$ & Low \\
\hline Bicer et $\mathrm{al}^{\mathrm{E} 9}$ & 2014 & $\begin{array}{l}\text { Apoptosis, } \\
\text { inflammation, and } \\
\text { oxidative stress }\end{array}$ & $\begin{array}{l}\text { OPCAB }(\mathrm{n}=25) \\
\text { CABG }(\mathrm{n}=25)\end{array}$ & $\begin{array}{l}57.7 \pm 8.4 \\
56.9 \pm 10.7\end{array}$ & NR & $\begin{array}{l}100 \\
100\end{array}$ & $\begin{array}{l}2.53 \pm 1.04 \\
2.57 \pm 0.83\end{array}$ & Unclear \\
\hline Blacher et $\mathrm{al}^{\mathrm{E} 10}$ & 2004 & Lymphocyte activation & $\begin{array}{l}\text { OPCAB }(n=13) \\
\text { CABG }(n=15)\end{array}$ & $\begin{array}{l}63 \pm 7 \\
61 \pm 10\end{array}$ & NR & $\begin{array}{l}100 \\
100\end{array}$ & $\begin{array}{l}2.6 \pm 0.5 \\
2.7 \pm 1.0\end{array}$ & Unclear \\
\hline Bonacchi et $\mathrm{al}^{\mathrm{E} 11}$ & 2006 & Cerebral injury markers & OPCAB $(n=18)$ & $63.7 \pm 5.4$ & $\begin{array}{l}>50 \% \text { MV-CAD; } \\
\text { LM } 16.7 \% \\
>50 \% \text { MV-CAD; } \\
\text { LM } 21 \%\end{array}$ & 100 & $\begin{array}{l}2.8 \pm 0.7 \\
3.1 \pm 0.6\end{array}$ & Unclear \\
\hline Caputo et $\mathrm{al}^{\mathrm{E} 12}$ & 2002 & $\begin{array}{l}\text { Inflammatory response } \\
\text { and organ function }\end{array}$ & $\begin{array}{l}\text { OPCAB }(\mathrm{n}=20) \\
\text { CABG }(\mathrm{n}=20)\end{array}$ & $\begin{array}{l}62.7 \pm 10.6 \\
62.9 \pm 6.9\end{array}$ & NR & $\begin{array}{l}100 \\
100\end{array}$ & $\begin{array}{l}2.8(2.4-3.0) \ddagger \\
2.9(2.6-3.2) \ddagger\end{array}$ & Low \\
\hline Carrier et $\mathrm{al}^{\mathrm{E} 13}$ & 2003 & $\begin{array}{l}\text { Hospital mortality } \\
\text { and morbidity }\end{array}$ & $\begin{array}{l}\operatorname{OPCAB}(\mathrm{n}=32) \\
\operatorname{CABG}(\mathrm{n}=33)\end{array}$ & $\begin{array}{l}70 \pm 8 \\
70 \pm 6\end{array}$ & $\begin{array}{l}>50 \% \text { MV-CAD; } \\
\text { LM } 29 \% \\
>50 \% \text { MV-CAD; } \\
\quad \text { LM } 32 \%\end{array}$ & 100 & $\begin{array}{r}3 \pm 1 \\
3.4 \pm 1\end{array}$ & Unclear \\
\hline Cavalca et $\mathrm{al}^{\mathrm{E} 14}$ & 2006 & $\begin{array}{l}\text { Isoprostanes and } \\
\text { oxidative stress }\end{array}$ & $\begin{array}{l}\text { OPCAB }(\mathrm{n}=25) \\
\text { CABG }(\mathrm{n}=25)\end{array}$ & $\begin{array}{l}68.4 \pm 8.9 \\
63.4 \pm 11.5\end{array}$ & NR & $\begin{array}{l}100 \\
100\end{array}$ & $\begin{array}{l}2.5 \pm 0.67 \\
3.1 \pm 0.67\end{array}$ & Unclear \\
\hline Chowdhury et al ${ }^{\mathrm{E} 15}$ & 2008 & $\begin{array}{l}\text { Periprocedural } \\
\text { inflammatory and } \\
\text { myocardial injury } \\
\text { marker release }\end{array}$ & $\begin{array}{l}\operatorname{OPCAB}(n=25) \\
\text { CABG }(n=25)\end{array}$ & $\begin{array}{l}58.8 \pm 7.8 \\
60.2 \pm 6.8\end{array}$ & $\begin{array}{l}>50 \% \text { MV-CAD } \\
>50 \% \text { MV-CAD }\end{array}$ & $\begin{array}{l}88.0 \\
76.0\end{array}$ & $\begin{array}{l}3.1 \pm 0.60 \\
3.2 \pm 0.62\end{array}$ & Unclear \\
\hline
\end{tabular}


TABLE 1. Continued

\begin{tabular}{|c|c|c|c|c|c|c|c|c|}
\hline Study ${ }^{\text {reference }}$ & Year & Primary endpoint & $\begin{array}{c}\text { Randomization } \\
\text { (ITT) } \\
\end{array}$ & $\begin{array}{c}\text { Age, } y, \\
\text { mean } \pm \text { SD }\end{array}$ & Extent of CAD & $\begin{array}{c}\text { IMA use, } \\
\% \% \\
\end{array}$ & $\begin{array}{l}\text { No. of } \\
\text { grafts* }\end{array}$ & $\begin{array}{c}\begin{array}{c}\text { Risk of } \\
\text { bias } \dagger\end{array} \\
\end{array}$ \\
\hline \multirow[t]{2}{*}{$\begin{array}{l}\text { Lamy et al } \\
\qquad{\text { [CORONARY }]^{\mathrm{E} 16}}^{\text {CORON }}\end{array}$} & \multirow[t]{2}{*}{2012} & \multirow{2}{*}{$\begin{array}{l}\text { Death, nonfatal stroke, } \\
\text { nonfatal MI, or } \\
\text { new renal failure } \\
\text { requiring dialysis } \\
\text { at } 30 \mathrm{~d} \text { after } \\
\text { randomization }\end{array}$} & $\operatorname{OPCAB}(\mathrm{n}=2375)$ & $67.6 \pm 6.7$ & $\begin{array}{l}\text { LM } 22.1 \% ;>50 \% \\
\text { MV-CAD }\end{array}$ & 93.6 & $3.0(0.95) \ddagger$ & \multirow[t]{2}{*}{ Low } \\
\hline & & & CABG $(n=2377)$ & $67.5 \pm 6.9$ & $\begin{array}{l}\text { LM } 20.9 \%>50 \% \\
\text { MV-CAD }\end{array}$ & 93.4 & $3.2(0.92) \ddagger$ & \\
\hline \multirow[t]{2}{*}{ Covino et $\mathrm{al}^{\mathrm{E} 17}$} & \multirow[t]{2}{*}{2001} & \multirow{2}{*}{$\begin{array}{l}\text { Length of operation, } \\
\text { hematologic and } \\
\text { biochemical } \\
\text { parameters, hemogas } \\
\text { analysis, volume of } \\
\text { blood loss, length } \\
\text { of stay in ICU }\end{array}$} & OPCAB $(n=21)$ & \multirow[t]{2}{*}{ NR } & $\begin{array}{l}>50 \% \\
\quad \text { MV-CAD }\end{array}$ & \multirow[t]{2}{*}{ NR } & 1.5 & \multirow[t]{2}{*}{ Unclear } \\
\hline & & & CABG $(n=16)$ & & $>50 \%$ MV-CAD & & 1.8 & \\
\hline \multirow[t]{2}{*}{$\begin{array}{l}\text { Rogers et al } \\
\qquad[\text { CRISP }]^{\mathrm{E} 18}\end{array}$} & \multirow[t]{2}{*}{2014} & \multirow{2}{*}{$\begin{array}{l}\text { All-cause death, new- } \\
\text { onset renal failure, } \\
\text { MI, prolonged } \\
\text { initial ventilation, } \\
\text { or sternal wound } \\
\text { dehiscence }\end{array}$} & OPCAB $(\mathrm{n}=53)$ & $76.4 \pm 5.8$ & $\begin{array}{l}\text { LM } 39 \%>50 \% \\
\text { MV-CAD }\end{array}$ & 96.2 & 2.56 & \multirow[t]{2}{*}{ Low } \\
\hline & & & CABG $(n=53)$ & $75.7 \pm 7.7$ & $\begin{array}{l}\text { LM } 27 \%>50 \% \\
\quad \text { MV-CAD }\end{array}$ & 83.0 & 2.68 & \\
\hline Czerny et $\mathrm{al}^{\mathrm{E} 19}$ & 2000 & $\begin{array}{l}\text { Periprocedural } \\
\text { inflammatory and } \\
\text { myocardial injury } \\
\text { marker release }\end{array}$ & $\begin{array}{l}\operatorname{OPCAB}(\mathrm{n}=15) \\
\text { CABG }(\mathrm{n}=15)\end{array}$ & $\begin{array}{l}63.3 \pm 9.3 \\
65.1 \pm 11.8\end{array}$ & $\begin{array}{l}>50 \% \text { MV-CAD } \\
>50 \% \text { MV-CAD }\end{array}$ & $\begin{array}{l}100 \\
100\end{array}$ & $\begin{array}{l}2.4 \pm 0.5 \\
3.4 \pm 0.9\end{array}$ & Unclear \\
\hline Czerny et al ${ }^{\mathrm{E} 20}$ & 2001 & $\begin{array}{l}\text { Completeness of } \\
\text { revascularization }\end{array}$ & $\begin{array}{l}\text { OPCAB }(n=40) \\
\text { CABG }(n=40)\end{array}$ & $\begin{array}{l}64.7 \pm 10.6 \\
62.3 \pm 9.9\end{array}$ & $\begin{array}{l}>50 \% \mathrm{MV}-\mathrm{CAD} \\
>50 \% \mathrm{MV}-\mathrm{CAD}\end{array}$ & $\begin{array}{l}100 \\
100\end{array}$ & $\begin{array}{l}2.6 \pm 0.5 \\
3.1 \pm 0.8\end{array}$ & Unclear \\
\hline Diegeler et al ${ }^{\mathrm{E} 21}$ & 2000 & $\begin{array}{l}\text { Periprocedural } \\
\text { neurocognitive } \\
\text { functioning }\end{array}$ & $\begin{array}{l}\text { OPCAB }(\mathrm{n}=20) \\
\text { CABG }(\mathrm{n}=20)\end{array}$ & $\begin{array}{l}65.6 \pm 6.4 \\
63.8 \pm 7.7\end{array}$ & $\begin{array}{l}>50 \% \text { MV-CAD } \\
>50 \% \text { MV-CAD }\end{array}$ & $\begin{array}{l}100 \\
100\end{array}$ & $\begin{array}{l}1.1 \pm 0.2 \\
1.1 \pm 0.5\end{array}$ & Unclear \\
\hline Dorman et al ${ }^{\mathrm{E} 22}$ & 2004 & $\begin{array}{l}\text { Endothelin plasma } \\
\text { content }\end{array}$ & $\begin{array}{l}\text { OPCAB }(n=27) \\
\text { CABG }(n=25)\end{array}$ & $\begin{array}{l}65 \pm 11 \\
61 \pm 3\end{array}$ & NR & NR & $\begin{array}{l}3 \pm 1 \\
4 \pm 1\end{array}$ & Unclear \\
\hline \multirow[t]{2}{*}{$\begin{array}{l}\text { Houlind et al } \\
\qquad{\text { [DOORS }]^{\mathrm{E} 23}}^{\text {DO }}\end{array}$} & \multirow[t]{2}{*}{2012} & \multirow[t]{2}{*}{$\begin{array}{l}\text { Death, stroke, or MI } \\
\text { at } 30 \mathrm{~d}\end{array}$} & OPCAB $(n=450)$ & $75(70-88) \ddagger$ & $\begin{array}{l}\text { LM } 45.0 \% ;>50 \% \\
\text { MV-CAD }\end{array}$ & 91.0 & $2.9 \pm 0.9$ & \multirow[t]{2}{*}{ Low } \\
\hline & & & CABG $(n=450)$ & $75(70-91) \ddagger$ & $\begin{array}{l}\text { LM } 48.0 \% ;>50 \% \\
\text { MV-CAD }\end{array}$ & 93.0 & $3.1 \pm 1.0$ & \\
\hline \multirow[t]{2}{*}{ Fattouch et al ${ }^{\mathrm{E} 24}$} & 2009 & $\begin{array}{l}\text { In-hospital death, } \\
\text { LCOS, prolonged }\end{array}$ & OPCAB $(n=63)$ & $63 \pm 16$ & $\begin{array}{l}\text { STEMI } 100 \% \\
\text { LM } 19 \%\end{array}$ & 93.6 & $2.6 \pm 0.5$ & Low \\
\hline & & $\begin{array}{l}\text { mechanical and } \\
\text { pharmacologic } \\
\text { cardiac support, } \\
\text { prolonged mechanical } \\
\text { ventilation support, } \\
\text { and postoperative } \\
\text { length of stay in } \\
\text { intensive care unit } \\
\text { and hospital }\end{array}$ & CABG $(n=65)$ & $61 \pm 18$ & $\begin{array}{l}\text { STEMI } 100 \% ; \\
\text { LM } 23 \%\end{array}$ & 92 & $2.8 \pm 0.4$ & \\
\hline Formica et al ${ }^{\mathrm{E} 25}$ & 2013 & $\begin{array}{l}\text { Periprocedural } \\
\text { inflammatory }\end{array}$ & OPCAB $(n=22)$ & $70.8 \pm 7.0$ & $\begin{array}{l}>50 \% \text { MV-CAD; } \\
\text { LM } 25 \%\end{array}$ & 100 & $2.7 \pm 0.5$ & Unclear \\
\hline & & marker release & CABG $(n=20)$ & $68.3 \pm 9.0$ & $\begin{array}{l}>50 \% \text { MV-CAD; } \\
\text { LM } 35 \%\end{array}$ & 100 & $2.7 \pm 0.5$ & \\
\hline Gasz et al ${ }^{\mathrm{E} 26}$ & 2004 & $\begin{array}{l}\text { Periprocedural } \\
\text { inflammatory } \\
\text { marker release }\end{array}$ & $\begin{array}{l}\text { OPCAB }(\mathrm{n}=10) \\
\text { CABG }(\mathrm{n}=10)\end{array}$ & $\begin{array}{l}63.4 \pm 2.8 \\
63.1 \pm 2.1\end{array}$ & NR & NR & $\begin{array}{l}3.4 \pm 0.31 \\
3.9 \pm 0.18\end{array}$ & Unclear \\
\hline Gasz et al ${ }^{\mathrm{E} 27}$ & 2005 & $\begin{array}{l}\text { Periprocedural } \\
\text { inflammatory } \\
\text { marker release } \\
\end{array}$ & $\begin{array}{l}\text { OPCAB }(n=10) \\
\text { CABG }(n=20)\end{array}$ & $63.2 \pm 2.5$ & NR & $\begin{array}{l}92 \\
88\end{array}$ & $\begin{array}{r}3.0 \pm 0.42 \\
3.21 \pm 0.22\end{array}$ & Unclear \\
\hline
\end{tabular}


TABLE 1. Continued

\begin{tabular}{|c|c|c|c|c|c|c|c|c|}
\hline Study ${ }^{\text {reference }}$ & Year & Primary endpoint & $\begin{array}{c}\text { Randomization } \\
\text { (ITT) }\end{array}$ & $\begin{array}{c}\text { Age, } y, \\
\text { mean } \pm \text { SD }\end{array}$ & Extent of CAD & $\begin{array}{c}\text { IMA use, } \\
\%\end{array}$ & $\begin{array}{l}\text { No. of } \\
\text { grafts* }\end{array}$ & $\begin{array}{c}\text { Risk of } \\
\text { bias } \dagger\end{array}$ \\
\hline Gerola et a ${ }^{\mathrm{E} 28}$ & 2004 & $\begin{array}{l}\text { Periprocedural all-cause } \\
\text { mortality, MI, } \\
\text { pulmonary } \\
\text { complications, } \\
\text { bleeding, wound } \\
\text { complications, } \\
\text { neurocognitive } \\
\text { dysfunction }\end{array}$ & $\begin{array}{l}\text { OPCAB }(\mathrm{n}=80) \\
\text { CABG }(\mathrm{n}=80)\end{array}$ & $\begin{array}{l}59.1 \pm 9.7 \\
58.9 \pm 8.9\end{array}$ & NR & $\begin{array}{l}98.7 \\
97.5\end{array}$ & $\begin{array}{l}1.77 \pm 0.68 \\
1.81 \pm 0.6\end{array}$ & Low \\
\hline $\mathrm{Gu}$ et $\mathrm{al}^{\mathrm{E} 29}$ & 1998 & $\begin{array}{l}\text { Inflammatory response } \\
\text { marker levels }\end{array}$ & $\begin{array}{l}\text { OPCAB }(n=31) \\
\text { CABG }(n=31)\end{array}$ & $\begin{array}{l}61.0 \pm 10.7 \\
60.0 \pm 8.5\end{array}$ & $\begin{array}{l}>50 \% \text { SV-CAD } \\
>50 \% \text { SV-CAD }\end{array}$ & $\begin{array}{l}100 \\
100\end{array}$ & $\begin{array}{l}1 \\
1\end{array}$ & Unclear \\
\hline Guler et $\mathrm{al}^{\mathrm{E} 30}$ & 2001 & $\begin{array}{l}\text { Postoperative lung } \\
\text { function }\end{array}$ & $\begin{array}{l}\operatorname{OPCAB}(\mathrm{n}=40) \\
\text { CABG }(\mathrm{n}=18)\end{array}$ & $\begin{array}{c}57.9 \pm 10.6 \\
52.3 \pm 8.68 \\
54.05 \pm 9.03\end{array}$ & $>50 \%$ SV-CAD & 100 & 1 & High \\
\hline Gonenc et $\mathrm{al}^{\mathrm{E} 31}$ & 2006 & $\begin{array}{l}\text { Periprocedural } \\
\text { oxidative stress }\end{array}$ & $\begin{array}{l}\text { OPCAB }(\mathrm{n}=30) \\
\text { CABG }(\mathrm{n}=12)\end{array}$ & $64.6 \pm 1.2$ & $\mathrm{NR}$ & NR & NA & High \\
\hline $\begin{array}{l}\text { Diegeler et al } \\
\qquad[\mathrm{GOPCABE}]^{\mathrm{E} 32}\end{array}$ & 2013 & $\begin{array}{l}\text { Composite of death, } \\
\text { stroke, MI, repeat } \\
\text { revascularization, } \\
\text { or new renal } \\
\text { replacement therapy } \\
\text { at } 30 \mathrm{~d} \text { and at } 12 \text { mo } \\
\text { after surgery }\end{array}$ & $\begin{array}{l}\operatorname{OPCAB}(\mathrm{n}=1271) \\
\operatorname{CABG}(\mathrm{n}=1268)\end{array}$ & $\begin{array}{l}78.6 \pm 3.0 \\
78.4 \pm 2.9\end{array}$ & $\begin{array}{l}>50 \% \text { MV-CAD; } \\
\text { LM } 28.1 \% \\
>50 \% \text { MV-CAD; } \\
\text { LM } 29.6 \%\end{array}$ & NR & 2.8 & Low \\
\hline 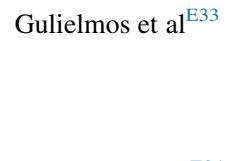 & 2000 & $\begin{array}{l}\text { Periprocedural } \\
\text { inflammatory marker } \\
\text { and cTn release }\end{array}$ & $\begin{array}{l}\text { OPCAB }(n=20) \\
\text { CABG }(n=20)\end{array}$ & $\begin{array}{l}65.1 \pm 10.0 \\
62.9 \pm 9.8 \S \\
59.6 \pm 11.0 \\
61.2 \pm 10.0 \S\end{array}$ & $\begin{array}{l}>50 \% \text { MV-CAD } \\
>50 \% \text { MV-CAD }\end{array}$ & 100 & 1 & Unclear \\
\hline Hernandez et $\mathrm{al}^{\mathrm{E} 34}$ & 2007 & $\begin{array}{l}\text { Neurocognitive } \\
\text { functioning at } \\
\text { discharge }\end{array}$ & $\begin{array}{l}\text { OPCAB }(n=99) \\
\text { CABG }(n=102)\end{array}$ & $\mathrm{NR}$ & $\begin{array}{l}\text { LM } 22.2 \% ;>50 \% \\
\text { MV-CAD } \\
\text { LM } 28.4 \% ;>50 \% \\
\text { MV-CAD }\end{array}$ & $\begin{array}{l}97.0 \\
98.0\end{array}$ & $\begin{array}{l}3.2 \pm 1.0 \\
3.3 \pm 0.9\end{array}$ & Low \\
\hline Hoel et $\mathrm{al}^{\mathrm{E} 35}$ & 2007 & Complement activation & $\begin{array}{l}\text { OPCAB }(n=22) \\
\text { CABG }(n=22)\end{array}$ & NR & NR & $\begin{array}{l}100 \\
100\end{array}$ & NA & Unclear \\
\hline Iqbal et $\mathrm{al}^{\mathrm{E} 36}$ & 2014 & $\begin{array}{l}\text { Neurologic } \\
\text { complications }\end{array}$ & $\begin{array}{l}\text { OPCAB }(\mathrm{n}=100) \\
\text { CABG }(\mathrm{n}=100)\end{array}$ & $\begin{array}{l}51.6 \pm 10.3 \\
53.5 \pm 10.0\end{array}$ & NR & NR & $\begin{array}{l}2.96 \pm 0.94 \\
2.99 \pm 0.88\end{array}$ & High \\
\hline Jares et $\mathrm{al}^{\mathrm{E} 37, \mathrm{E} 104}$ & 2007 & $\begin{array}{l}\text { Identification of } \\
\text { fibrinolysis by } \\
\text { rotation } \\
\text { thromboelastography }\end{array}$ & $\begin{array}{l}\text { OPCAB }(n=10) \\
\text { CABG }(n=10)\end{array}$ & $\begin{array}{l}65.5 \\
62.6\end{array}$ & NR & NR & $\begin{array}{l}2.00 \\
2.60\end{array}$ & Unclear \\
\hline 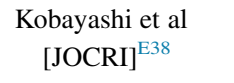 & 2005 & $\begin{array}{l}\text { Cardiac death, MI, } \\
\text { CHF, or TVR at } 3 \mathrm{y}\end{array}$ & $\begin{array}{l}\operatorname{OPCAB}(\mathrm{n}=81) \\
\mathrm{CABG}(\mathrm{n}=86)\end{array}$ & $\begin{array}{l}60 \pm 7 \\
59 \pm 10\end{array}$ & $\begin{array}{l}>50 \% \mathrm{MV}-\mathrm{CAD} \\
>50 \% \mathrm{MV}-\mathrm{CAD}\end{array}$ & $\begin{array}{l}33.6 \\
31.5\end{array}$ & $\begin{array}{l}3.5 \pm 1.0 \\
3.6 \pm 0.9\end{array}$ & Low \\
\hline 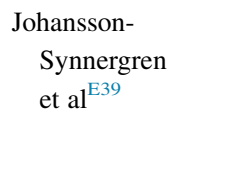 & 2004 & $\begin{array}{l}\text { Periprocedural } \\
\text { inflammatory } \\
\text { marker release } \\
\text { and endothelial } \\
\text { function }\end{array}$ & $\begin{array}{l}\operatorname{OPCAB}(n=26) \\
\text { CABG }(n=26)\end{array}$ & $\begin{array}{l}62.2 \pm 1.7 \\
62.5 \pm 1.9\end{array}$ & $\begin{array}{l}\text { NR } \\
\text { NR }\end{array}$ & $\begin{array}{l}96.2 \\
96.2\end{array}$ & $\begin{array}{l}1.8 \pm 0.1 \\
2.2 \pm 0.1\end{array}$ & Unclear \\
\hline Jongman et $\mathrm{al}^{\mathrm{E} 40}$ & 2014 & $\begin{array}{l}\text { Systemic inflammation } \\
\text { markers and } \\
\text { endothelial response }\end{array}$ & $\begin{array}{l}\text { OPCAB }(\mathrm{n}=30) \\
\text { CABG }(\mathrm{n}=30)\end{array}$ & $\begin{array}{l}63(47-80) \ddagger \\
63(47-77) \ddagger\end{array}$ & NR & NR & $\begin{array}{l}3(2-5) \ddagger \\
3(2-5) \ddagger\end{array}$ & Unclear \\
\hline Khan et $\mathrm{al}^{\mathrm{E} 41}$ & 2004 & Graft patency at $3 \mathrm{mo}$ & $\begin{array}{l}\text { OPCAB }(\mathrm{n}=54) \\
\text { CABG }(\mathrm{n}=50)\end{array}$ & $\begin{array}{l}62.0 \pm 7.9 \\
64.7 \pm 8.4\end{array}$ & $\begin{array}{l}>50 \% \text { MV-CAD } \\
>50 \% \text { MV-CAD }\end{array}$ & $\begin{array}{l}96 \\
94\end{array}$ & $\begin{array}{l}3.1 \pm 0.6 \\
3.4 \pm 0.7\end{array}$ & Low \\
\hline Kochamba et al ${ }^{\mathrm{E} 42}$ & 2000 & $\begin{array}{l}\text { Preoperative and } \\
\text { postoperative } \\
\text { pulmonary } \\
\text { gas exchange }\end{array}$ & $\begin{array}{l}\text { OPCAB }(n=29) \\
\text { CABG }(n=29)\end{array}$ & $\begin{array}{l}55.6 \pm 9.5 \\
61.6 \pm 10.0\end{array}$ & NR & $\begin{array}{l}97.0 \\
93.0\end{array}$ & $\begin{array}{l}1.5 \\
1.6\end{array}$ & Unclear \\
\hline
\end{tabular}


TABLE 1. Continued

\begin{tabular}{|c|c|c|c|c|c|c|c|c|}
\hline Study ${ }^{\text {reference }}$ & Year & Primary endpoint & $\begin{array}{c}\text { Randomization } \\
\text { (ITT) }\end{array}$ & $\begin{array}{c}\text { Age, } y \\
\text { mean } \pm \text { SD }\end{array}$ & Extent of CAD & $\begin{array}{c}\text { IMA use, } \\
\%\end{array}$ & $\begin{array}{l}\text { No. of } \\
\text { grafts* }\end{array}$ & $\begin{array}{c}\text { Risk of } \\
\text { bias }\end{array}$ \\
\hline Kok et al ${ }^{\mathrm{E} 43}$ & 2014 & $\begin{array}{l}\text { Cerebral tissue } \\
\text { oxygenation and } \\
\text { postoperative cognitive } \\
\text { dysfunction }\end{array}$ & $\begin{array}{l}\operatorname{OPCAB}(\mathrm{n}=30) \\
\operatorname{CABG}(\mathrm{n}=30)\end{array}$ & $\begin{array}{l}63.0 \pm 9.0 \\
62.6 \pm 9.9\end{array}$ & NR & NR & $\begin{array}{r}3.2 \pm 0.9 \\
3.3 . \pm 0.8\end{array}$ & Unclear \\
\hline Krejca et $\mathrm{al}^{\mathrm{E} 44}$ & 1999 & $\begin{array}{l}\text { Periprocedural } \\
\text { inflammatory marker } \\
\text { release }\end{array}$ & $\begin{array}{l}\operatorname{OPCAB}(n=13) \\
\text { CABG }(n=13)\end{array}$ & $\begin{array}{l}54.1 \pm 9.6 \\
56.2 \pm 5.3\end{array}$ & NR & NR & $\begin{array}{l}1.8(1-2) \ddagger \\
1.8(1-2) \ddagger\end{array}$ & Unclear \\
\hline Kunes et $\mathrm{al}^{\mathrm{E} 45}$ & 2007 & $\begin{array}{l}\text { Pentraxin } 3 \text { release } \\
\text { kinetics }\end{array}$ & $\begin{array}{l}\text { OPCAB }(\mathrm{n}=17) \\
\text { CABG }(\mathrm{n}=17)\end{array}$ & $\begin{array}{l}66.4 \pm 10.1 \\
70.5 \pm 7.1\end{array}$ & NR & NR & $\begin{array}{l}2.0 \\
2.0\end{array}$ & Unclear \\
\hline Lee et $\mathrm{al}^{\mathrm{E} 46}$ & 2003 & $\begin{array}{l}\text { Inhospital all-cause death, } \\
\text { stroke, and length of } \\
\text { stay, intra-aortic } \\
\text { balloon support } \\
\text { postoperatively }\end{array}$ & $\begin{array}{l}\text { OPCAB }(\mathrm{n}=30) \\
\text { CABG }(\mathrm{n}=30)\end{array}$ & $\begin{array}{l}65.5 \pm 9.6 \\
66.0 \pm 11.2\end{array}$ & NR & NR & $\begin{array}{l}3.1 \pm 0.7 \\
3.6 \pm 0.9\end{array}$ & Unclear \\
\hline Legare et $\mathrm{al}^{\mathrm{E} 47}$ & 2004 & $\begin{array}{l}\text { Periprocedural death, } \\
\text { MI, stroke, AF, deep } \\
\text { sternal wound infection }\end{array}$ & $\begin{array}{l}\text { OPCAB }(n=150) \\
\text { CABG }(n=150)\end{array}$ & $\begin{array}{l}62.1 \pm 10.1 \\
63.7 \pm 10.0\end{array}$ & $\begin{array}{l}\text { LM } 10.7 \% ;>50 \% \\
\text { MV-CAD } \\
\text { LM } 9.3 \% ;>50 \% \\
\text { MV-CAD }\end{array}$ & 96.7 & $\begin{array}{l}2.8 \pm 0.9 \\
3.0 \pm 0.9\end{array}$ & Low \\
\hline Lingaas et $\mathrm{al}^{\mathrm{E} 48, \mathrm{E} 49}$ & 2004 & Graft patency at $12 \mathrm{mo}$ & $\mathrm{CABG}(\mathrm{n}=60)$ & $\begin{array}{l}64 \pm 7.8 \\
65 \pm 8.3\end{array}$ & $\begin{array}{l}\text { LM } 10.0 \% ;>50 \% \\
\text { MV-CAD } \\
\text { LM 5.0\%; }>50 \% \\
\text { MV-CAD }\end{array}$ & 100 & $\begin{array}{l}2.6 \pm 0.9 \\
2.8 \pm 1.0\end{array}$ & Unclear \\
\hline Lloyd et $\mathrm{al}^{\mathrm{E} 50}$ & 2000 & $\begin{array}{l}\text { Serum S-100 protein and } \\
\text { neuropsychological } \\
\text { outcomes }\end{array}$ & $\begin{array}{l}\operatorname{OPCAB}(n=63) \\
\text { CABG }(n=62)\end{array}$ & $\begin{array}{l}59(54-64) \ddagger \\
62(56-69) \ddagger\end{array}$ & $\begin{array}{l}>50 \% \mathrm{MV}-\mathrm{CAD} \\
>50 \% \mathrm{MV}-\mathrm{CAD}\end{array}$ & NR & $\begin{array}{l}2.2 \pm 0.9 \\
2.4 \pm 0.9\end{array}$ & Unclear \\
\hline Lund et $\mathrm{al}^{\mathrm{E} 51, \mathrm{E} 52}$ & 2003 & $\begin{array}{l}\text { Intraoperative cerebral } \\
\text { embolization }\end{array}$ & $\begin{array}{l}\text { OPCAB }(\mathrm{n}=29) \\
\text { CABG }(\mathrm{n}=23)\end{array}$ & $\begin{array}{l}62.0(52-73) \ddagger \\
64.0(52-79) \ddagger\end{array}$ & NR & $\begin{array}{l}100 \\
100\end{array}$ & $\begin{array}{l}2.3 \pm 0.7 \\
2.5 \pm 0.8\end{array}$ & Unclear \\
\hline Malik et $\mathrm{al}^{\mathrm{E} 53}$ & 2006 & $\begin{array}{l}\text { Periprocedural } \\
\text { CPK-MB release }\end{array}$ & $\begin{array}{l}\text { OPCAB }(n=25) \\
\text { CABG }(n=25)\end{array}$ & $\begin{array}{l}56.4 \pm 8.8 \\
59.8 \pm 6.1\end{array}$ & $\begin{array}{l}>50 \% \text { MV-CAD } \\
>50 \% \text { MV-CAD }\end{array}$ & $\begin{array}{l}80 \\
60\end{array}$ & $\begin{array}{l}3.1 \pm 0.58 \\
3.1 \pm 0.5\end{array}$ & Unclear \\
\hline Mandak $\mathrm{J}$ et $\mathrm{al}^{\mathrm{E} 54}$ & 2008 & $\begin{array}{l}\text { Peripheral tissue } \\
\text { metabolism and } \\
\text { microvascular blood } \\
\text { flow }\end{array}$ & $\begin{array}{l}\operatorname{OPCAB}(\mathrm{n}=20) \\
\operatorname{CABG}(\mathrm{n}=20)\end{array}$ & $\begin{array}{l}66.3 \pm 7.0 \\
67.0 \pm 7.3\end{array}$ & NR & $\begin{array}{l}100 \\
100\end{array}$ & $\begin{array}{l}2.4 \pm 0.8 \\
2.9 \pm 0.7\end{array}$ & Unclear \\
\hline Matata et $\mathrm{al}^{\mathrm{E} 55}$ & 2000 & $\begin{array}{l}\text { Periprocedural } \\
\text { inflammatory } \\
\text { marker release and } \\
\text { oxidative stress }\end{array}$ & $\begin{array}{l}\text { OPCAB }(n=10) \\
\text { CABG }(n=10)\end{array}$ & $\begin{array}{l}59.5 \pm 2.2 \\
59.5 \pm 2.7\end{array}$ & NR & NR & $\begin{array}{l}1.8 \pm 0.2 \\
1.9 \pm 0.2\end{array}$ & Low \\
\hline $\begin{array}{l}\text { Hueb et al } \\
\qquad\left[\text { MASS III] }{ }^{\mathrm{E} 56}\right.\end{array}$ & 2010 & $\begin{array}{l}\text { Freedom from overall } \\
\text { mortality, stroke, } \\
\text { MI, and additional } \\
\text { revascularization }\end{array}$ & $\begin{array}{l}\text { OPCAB }(n=156) \\
\text { CABG }(n=155)\end{array}$ & $\begin{array}{l}61 \\
59\end{array}$ & $\begin{array}{l}>50 \% \text { MV-CAD } \\
>50 \% \text { MV-CAD }\end{array}$ & $\begin{array}{l}98 \\
94\end{array}$ & $\begin{array}{l}2.60 \\
3.18\end{array}$ & Low \\
\hline Medved et al ${ }^{\mathrm{E} 57}$ & 2008 & $\begin{array}{l}\text { In-hospital mortality } \\
\text { and morbidity }\end{array}$ & $\begin{array}{l}\operatorname{OPCAB}(\mathrm{n}=30) \\
\operatorname{CABG}(\mathrm{n}=30)\end{array}$ & $\begin{array}{r}59.3 \pm 7.2 \\
62 \pm 5.4\end{array}$ & NR & $\begin{array}{l}93.3 \\
90\end{array}$ & $\begin{array}{l}2.3 \pm 0.34 \\
2.5 \pm 0.58\end{array}$ & Unclear \\
\hline Michaux et $\mathrm{al}^{\mathrm{E} 58, \mathrm{E} 59}$ & 2011 & $\begin{array}{l}\text { Right ventricular global } \\
\text { and overall systolic } \\
\text { function }\end{array}$ & $\begin{array}{l}\text { OPCAB }(n=25) \\
\text { CABG }(n=25)\end{array}$ & $\begin{array}{l}61 \pm 9 \\
65 \pm 8\end{array}$ & $\begin{array}{l}>50 \% \text { MV-CAD } \\
>50 \% \text { MV-CAD }\end{array}$ & $\begin{array}{l}100 \\
100\end{array}$ & $\begin{array}{l}2.9 \pm 0.6 \\
3.2 \pm 0.7\end{array}$ & Low \\
\hline Modine et $\mathrm{al}^{\mathrm{E} 60}$ & 2009 & $\begin{array}{l}\text { Renal tubular and } \\
\text { glomerular function }\end{array}$ & $\begin{array}{l}\text { OPCAB }(\mathrm{n}=35) \\
\text { CABG }(\mathrm{n}=36)\end{array}$ & $\begin{array}{l}66.5 \pm 7.6 \\
62.6 \pm 9.6\end{array}$ & NR & $\begin{array}{l}100 \\
100\end{array}$ & $\begin{array}{l}2.4 \pm 0.55 \\
2.8 \pm 0.8\end{array}$ & Unclear \\
\hline $\begin{array}{l}\text { Motallebzadeh } \\
\text { et } \mathrm{al}^{\mathrm{E} 61}\end{array}$ & 2004 & $\begin{array}{l}\text { Periprocedural cerebral } \\
\text { injury }\end{array}$ & $\begin{array}{l}\text { OPCAB }(\mathrm{n}=15) \\
\text { CABG }(\mathrm{n}=20)\end{array}$ & $\begin{array}{l}65(53-72) \ddagger \\
63(59-70) \ddagger\end{array}$ & $\begin{array}{l}>50 \% \text { MV-CAD } \\
>50 \% \text { MV-CAD }\end{array}$ & NR & $\begin{array}{l}2.2 \pm 0.94 \\
3.2 \pm 0.99\end{array}$ & Low \\
\hline $\begin{array}{l}\text { Motallebzadeh } \\
\text { et } \mathrm{al}^{\mathrm{E} 62}\end{array}$ & 2007 & $\begin{array}{l}\text { Periprocedural } \\
\text { neurocognitive function }\end{array}$ & $\begin{array}{l}\operatorname{OPCAB}(\mathrm{n}=108) \\
\text { CABG }(\mathrm{n}=104)\end{array}$ & $\begin{array}{l}63.9 \pm 0.9 \\
65.1 \pm 0.9\end{array}$ & $\begin{array}{l}>50 \% \text { MV-CAD } \\
>50 \% \text { MV-CAD }\end{array}$ & NR & NR & Low \\
\hline
\end{tabular}


TABLE 1. Continued

\begin{tabular}{|c|c|c|c|c|c|c|c|c|}
\hline Study ${ }^{\text {reference }}$ & Year & Primary endpoint & $\begin{array}{c}\text { Randomization } \\
\text { (ITT) }\end{array}$ & $\begin{array}{c}\text { Age, } y \\
\text { mean } \pm \text { SD }\end{array}$ & Extent of CAD & $\begin{array}{c}\text { IMA use, } \\
\%\end{array}$ & $\begin{array}{l}\text { No. of } \\
\text { grafts* }\end{array}$ & $\begin{array}{c}\text { Risk of } \\
\text { bias } \dagger\end{array}$ \\
\hline \multirow[t]{2}{*}{ Muneretto et $\mathrm{al}^{\mathrm{E} 63}$} & \multirow[t]{2}{*}{2003} & \multirow{2}{*}{$\begin{array}{l}\text { Number of } \\
\text { anastomoses, mean } \\
\text { mechanical } \\
\text { ventilation time, ICU } \\
\text { and postoperative stay }\end{array}$} & OPCAB $(\mathrm{n}=88)$ & $67 \pm 8$ & $\begin{array}{c}\text { LM } 9 \%>50 \% \\
\text { MV-CAD }\end{array}$ & NR & $2.7 \pm 0.5$ & \multirow[t]{2}{*}{ Unclear } \\
\hline & & & CABG $(n=88)$ & $66 \pm 9$ & $\begin{array}{l}\text { LM } 11.3 \%>50 \% \\
\text { MV-CAD }\end{array}$ & & $2.8 \pm 0.8$ & \\
\hline Nesher et al ${ }^{\mathrm{E} 64}$ & 2006 & $\begin{array}{l}\text { Periprocedural } \\
\text { inflammatory marker } \\
\text { and CPK-MB release }\end{array}$ & $\begin{array}{l}\text { OPCAB }(n=61) \\
\text { CABG }(n=64)\end{array}$ & $\begin{array}{l}67 \pm 1 \\
68 \pm 5\end{array}$ & NR & NR & $\begin{array}{l}2.3 \pm 0.9 \\
2.9 \pm 1.5\end{array}$ & Low \\
\hline Niranjan et al ${ }^{\mathrm{E} 65}$ & 2006 & $\begin{array}{l}\text { Autologus blood } \\
\text { transfusion and } \\
\text { postoperative } \\
\text { complications }\end{array}$ & $\begin{array}{l}\text { OPCAB }(n=40) \\
\text { CABG }(n=40)\end{array}$ & $\begin{array}{l}67.6 \pm 10.5 \\
66.2 \pm 9.5\end{array}$ & $\begin{array}{l}>50 \% \mathrm{MV}-\mathrm{CAD} \\
>50 \% \mathrm{MV}-\mathrm{CAD}\end{array}$ & $\begin{array}{l}95.0 \\
97.5\end{array}$ & $\begin{array}{l}3.93 \pm 0.8 \\
3.75 \pm 0.8\end{array}$ & Low \\
\hline $\begin{array}{l}\text { Nathoe et al } \\
\text { [Octopus] }^{\mathrm{E} 66}\end{array}$ & 2003 & $\begin{array}{l}\text { Freedom from all- } \\
\text { cause death, stroke, } \\
\text { MI and repeat } \\
\text { revascularization }\end{array}$ & $\begin{array}{l}\text { OPCAB }(n=142) \\
\text { CABG }(n=139)\end{array}$ & $\begin{array}{l}61.7 \pm 9.2 \\
60.8 \pm 8.8\end{array}$ & $\begin{array}{l}>50 \% \mathrm{MV}-\mathrm{CAD} \\
>50 \% \mathrm{MV}-\mathrm{CAD}\end{array}$ & NR & $\begin{array}{l}2.4 \\
2.6\end{array}$ & Low \\
\hline $\begin{array}{l}\text { Lemma et al } \\
\qquad[\text { On-Off }]^{\mathrm{E} 67}\end{array}$ & 2012 & $\begin{array}{l}\text { Operative mortality, } \\
\text { MI, stroke, renal } \\
\text { failure, reoperation } \\
\text { for bleeding and } \\
\text { ARDS within } 30 \mathrm{~d}\end{array}$ & $\begin{array}{l}\text { OPCAB }(n=208) \\
\text { CABG }(n=203)\end{array}$ & $\begin{array}{l}74(69-78) \ddagger \\
73(68-77) \ddagger\end{array}$ & NR & $\begin{array}{l}99 \\
97\end{array}$ & $\begin{array}{l}3.0 \pm 1.1 \\
3.3 \pm 1.0\end{array}$ & Low \\
\hline Onorati et al ${ }^{\mathrm{E} 68}$ & 2010 & $\begin{array}{l}\text { Perioperative changes } \\
\text { in MCP-1 and } \\
\text { VEGF levels }\end{array}$ & $\begin{array}{l}\text { OPCAB }(n=20) \\
\text { CABG }(n=40)\end{array}$ & $\begin{array}{l}71.6 \pm 4.8 \\
71.7 \pm 4.4\end{array}$ & NR & NR & $\begin{array}{l}3.4 \pm 0.5 \\
3.4 \pm 0.6\end{array}$ & Unclear \\
\hline Ozkara et $\mathrm{al}^{\mathrm{E} 69}$ & 2007 & $\begin{array}{l}\text { Target vessel } \\
\text { revascularization at } \\
1 \mathrm{y} \text {; periprocedural } \\
\text { PAI-1 release }\end{array}$ & $\begin{array}{l}\operatorname{OPCAB}(n=22) \\
\text { CABG }(n=42)\end{array}$ & $\begin{array}{l}58.9 \pm 10.1 \\
59.5 \pm 9.9\end{array}$ & NR & $\begin{array}{l}100 \\
100\end{array}$ & $\begin{array}{l}2.48 \pm 0.52 \\
2.31 \pm 0.60\end{array}$ & Unclear \\
\hline Paparella et al ${ }^{\mathrm{E} 70}$ & 2006 & $\begin{array}{l}\text { Activation of the } \\
\text { coagulation and } \\
\text { fibrinolytic systems }\end{array}$ & $\begin{array}{l}\text { OPCAB }(\mathrm{n}=16) \\
\text { CABG }(\mathrm{n}=16)\end{array}$ & $\begin{array}{l}\mathrm{NR} \\
\mathrm{NR}\end{array}$ & NR & NR & $\begin{array}{c}2.7 \\
3.25\end{array}$ & Unclear \\
\hline Parolari et $\mathrm{al}^{\mathrm{E} 71}$ & 2003 & $\begin{array}{l}\text { Periprocedural } \\
\quad \text { oxygen metabolism }\end{array}$ & $\begin{array}{l}\operatorname{OPCAB}(n=11) \\
\operatorname{CABG}(n=14)\end{array}$ & $\begin{array}{l}60 \pm 2.1 \\
62 \pm 2.3\end{array}$ & NR & NR & $\begin{array}{l}2.3 \pm 0.14 \\
2.9 \pm 0.20\end{array}$ & Unclear \\
\hline Parolari et $\mathrm{al}^{\mathrm{E} 72}$ & 2005 & $\begin{array}{l}\text { Periprocedural plasma } \\
\text { P-selectin and TF } \\
\text { levels }\end{array}$ & $\begin{array}{l}\operatorname{OPCAB}(\mathrm{n}=15) \\
\mathrm{CABG}(\mathrm{n}=15)\end{array}$ & $\begin{array}{l}65 \pm 1.2 \\
64 \pm 1.4\end{array}$ & $\begin{array}{l}>50 \% \mathrm{MV}-\mathrm{CAD} \\
>50 \% \mathrm{MV}-\mathrm{CAD}\end{array}$ & NR & $\begin{array}{l}2.8 \pm 0.13 \\
2.9 \pm 0.16\end{array}$ & Unclear \\
\hline Penttilä et $\mathrm{al}^{\mathrm{E} 73}$ & 2001 & $\begin{array}{r}\text { Periprocedural changes in } \\
\text { myocardial metabolism }\end{array}$ & $\begin{array}{l}\text { OPCAB }(n=11) \\
\text { CABG }(n=11)\end{array}$ & $\begin{array}{l}59.5(53.3-65.8) \ddagger \\
59.2(53.0-65.4) \ddagger\end{array}$ & $\begin{array}{l}>50 \% \text { MV-CAD } \\
>50 \% \text { MV-CAD }\end{array}$ & NR & $\begin{array}{l}2.8(2.3-3.3) \ddagger \\
3.3(2.4-4.2) \ddagger\end{array}$ & Unclear \\
\hline $\begin{array}{l}\text { Straka et al } \\
\qquad \text { [PRAGUE-4] }^{\mathrm{E} 74}\end{array}$ & 2004 & $\begin{array}{l}\text { Death, MI, stroke, renal } \\
\text { failure requiring } \\
\text { hemodialysis at } 30 \mathrm{~d}\end{array}$ & $\begin{array}{l}\text { OPCAB }(n=192) \\
\text { CABG }(n=208)\end{array}$ & $\begin{array}{l}63 \\
62\end{array}$ & $\begin{array}{l}>50 \% \text { MV-CAD } \\
>50 \% \text { MV-CAD }\end{array}$ & $\begin{array}{l}97 \\
90\end{array}$ & $\begin{array}{l}2.3 \\
2.7\end{array}$ & Low \\
\hline $\begin{array}{l}\text { Hlavicka et al } \\
\qquad \text { [PRAGUE-6] }^{\mathrm{E} 75}\end{array}$ & 2013 & $\begin{array}{l}\text { Death, MI, stroke, renal } \\
\text { failure requiring } \\
\text { hemodialysis at } 30 \mathrm{~d}\end{array}$ & $\begin{array}{l}\operatorname{OPCAB}(\mathrm{n}=98) \\
\operatorname{CABG}(\mathrm{n}=108)\end{array}$ & $\begin{array}{l}74.7 \pm 6.5 \\
73.6 \pm 7.4\end{array}$ & NR & NR & $\begin{array}{l}2.04 \\
2.66\end{array}$ & Low \\
\hline $\begin{array}{l}\text { Bednar et al } \\
\quad[\text { PRAGUE 11] }]^{\mathrm{E} 76}\end{array}$ & 2008 & $\begin{array}{l}\text { Platelet activity and } \\
\text { aspirin efficacy }\end{array}$ & $\begin{array}{l}\operatorname{OPCAB}(n=40) \\
\operatorname{CABG}(n=40)\end{array}$ & $\begin{array}{l}68 \pm 9 \\
64 \pm 11\end{array}$ & NR & NR & $\begin{array}{l}1.9 \pm 0.7 \\
2.4 \pm 0.6\end{array}$ & Unclear \\
\hline 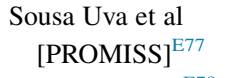 & 2010 & Graft patency at $5 \mathrm{wk}$ & $\begin{array}{l}\operatorname{OPCAB}(\mathrm{n}=75) \\
\mathrm{CABG}(\mathrm{n}=75)\end{array}$ & $\begin{array}{l}66.1 \pm 9.5 \\
64.6 \pm 9.8\end{array}$ & NR & $\begin{array}{l}100 \\
100\end{array}$ & $\begin{array}{l}3.5 \pm 0.6 \\
3.5 \pm 0.6\end{array}$ & Low \\
\hline Rachwalik et $\mathrm{al}^{\mathrm{E} 78}$ & 2006 & $\begin{array}{l}\text { Periprocedural } \\
\quad \text { respiratory function }\end{array}$ & $\begin{array}{l}\text { OPCAB }(n=21) \\
\text { CABG }(n=21)\end{array}$ & $\begin{array}{l}57.1 \pm 8.9 \\
95.3 \pm 7.9\end{array}$ & NR & $\begin{array}{l}100 \\
100\end{array}$ & NA & Unclear \\
\hline Rainio et $\mathrm{al}^{\mathrm{E} 79}$ & 2007 & $\begin{array}{l}\text { Periprocedural retinal } \\
\text { microembolism }\end{array}$ & $\begin{array}{l}\text { OPCAB }(n=10) \\
\text { CABG }(n=10)\end{array}$ & $\begin{array}{l}64.6 \pm 4 \\
58.6 \pm 2.6\end{array}$ & NR & $\begin{array}{l}100 \\
100\end{array}$ & $\begin{array}{l}4.1 \\
4.4\end{array}$ & Low \\
\hline
\end{tabular}


TABLE 1. Continued

\begin{tabular}{|c|c|c|c|c|c|c|c|c|}
\hline Study ${ }^{\text {reference }}$ & Year & Primary endpoint & $\begin{array}{c}\text { Randomization } \\
\text { (ITT) }\end{array}$ & $\begin{array}{c}\text { Age, } y, \\
\text { mean } \pm \text { SD }\end{array}$ & Extent of CAD & $\begin{array}{c}\text { IMA use, } \\
\%\end{array}$ & $\begin{array}{l}\text { No. of } \\
\text { grafts* }\end{array}$ & $\begin{array}{c}\text { Risk of } \\
\text { bias } \dagger\end{array}$ \\
\hline Raja et al ${ }^{\mathrm{E} 80}$ & 2003 & $\begin{array}{l}\text { Postoperative } \\
\text { gastrointestinal } \\
\text { complications }\end{array}$ & $\begin{array}{l}\text { OPCAB }(n=150) \\
\text { CABG }(n=150)\end{array}$ & NR & NR & $\begin{array}{l}98 \\
98\end{array}$ & $\begin{array}{l}2(1-4) \ddagger \\
2(1-4) \ddagger\end{array}$ & Unclear \\
\hline Rasmussen et al ${ }^{\mathrm{E} 81}$ & 2007 & $\begin{array}{l}\text { Periprocedural } \\
\text { inflammatory marker } \\
\text { release }\end{array}$ & $\begin{array}{l}\operatorname{OPCAB}(\mathrm{n}=17) \\
\mathrm{CABG}(\mathrm{n}=18)\end{array}$ & $\begin{array}{l}68 \pm 6 \\
67 \pm 4\end{array}$ & NR & $\begin{array}{l}94 \\
94\end{array}$ & $\begin{array}{l}3.1 \pm 0.9 \\
3.1 \pm 0.6\end{array}$ & Unclear \\
\hline Rastan et $\mathrm{al}^{\mathrm{E} 82}$ & 2005 & $\begin{array}{l}\text { Periprocedural } \\
\text { inflammatory marker, } \\
\text { CPK-MB, and cTn } \\
\text { release }\end{array}$ & $\begin{array}{l}\text { OPCAB }(n=20) \\
\text { CABG }(n=20)\end{array}$ & $\begin{array}{l}63.0 \pm 6.0 \\
65.3 \pm 3.9\end{array}$ & NR & $\begin{array}{l}100 \\
100\end{array}$ & $\begin{array}{l}3.0 \pm 0.5 \\
2.9 \pm 0.3\end{array}$ & Unclear \\
\hline $\begin{array}{l}\text { Schroyer et al } \\
\qquad[\mathrm{ROOBY}]^{\mathrm{E} 83}\end{array}$ & 2009 & $\begin{array}{l}\text { All-cause death, } \\
\text { reoperation, new } \\
\text { mechanical support, } \\
\text { coma, stroke, cardiac } \\
\text { arrest, renal failure } \\
\text { requiring dialysis } \\
\text { at } 30 \mathrm{~d}\end{array}$ & $\begin{array}{l}\text { OPCAB }(\mathrm{n}=1104) \\
\mathrm{CABG}(\mathrm{n}=1099)\end{array}$ & $\begin{array}{l}63.0 \pm 8.5 \\
62.5 \pm 8.5\end{array}$ & $\begin{array}{l}>50 \% \mathrm{MV}-\mathrm{CAD} \\
>50 \% \mathrm{MV}-\mathrm{CAD}\end{array}$ & $\begin{array}{l}\text { NR } \\
\text { NR }\end{array}$ & $\begin{array}{l}2.9 \pm 0.9 \\
3.0 \pm 1.0\end{array}$ & Low \\
\hline Sahlman et al ${ }^{\mathrm{E} 84}$ & 2003 & $\begin{array}{l}\text { Periprocedural } \\
\text { inflammatory and } \\
\text { CPK-MB release }\end{array}$ & $\begin{array}{l}\text { OPCAB }(n=24) \\
\text { CABG }(n=26)\end{array}$ & $\begin{array}{l}64.0 \pm 9.0 \\
61.5 \pm 8.1\end{array}$ & NR & $\begin{array}{l}100 \\
100\end{array}$ & $\begin{array}{l}3.2 \pm 0.9 \\
3.0 \pm 1.2\end{array}$ & Unclear \\
\hline Sajja et $\mathrm{al}^{\mathrm{E} 85}$ & 2007 & $\begin{array}{l}\text { Periprocedural renal } \\
\text { function }\end{array}$ & $\begin{array}{l}\text { OPCAB }(\mathrm{n}=56) \\
\text { CABG }(\mathrm{n}=60)\end{array}$ & $\begin{array}{l}60.0 \pm 8.4 \\
60.5 \pm 7.9\end{array}$ & NR & NR & $\begin{array}{l}3.11 \pm 0.89 \\
3.85 \pm 0.86\end{array}$ & Unclear \\
\hline Selvanayagam t al ${ }^{\mathrm{E} 86}$ & 2004 & Periprocedural LVEF & $\begin{array}{l}\text { OPCAB }(\mathrm{n}=30) \\
\text { CABG }(\mathrm{n}=30)\end{array}$ & $\begin{array}{l}60 \pm 9 \\
61 \pm 11\end{array}$ & NR & $\begin{array}{r}100 \\
97\end{array}$ & $\begin{array}{l}2.8 \pm 0.9 \\
2.9 \pm 0.8\end{array}$ & Low \\
\hline $\begin{array}{l}\text { Puskas et al } \\
\qquad{\text { [SMART }]^{\mathrm{E} 87}}^{\text {SMA }}\end{array}$ & 2003 & $\begin{array}{l}\text { Completeness of } \\
\text { revascularization } \\
\text { and graft patency } \\
\text { at } 30 \mathrm{~d}\end{array}$ & $\begin{array}{l}\text { OPCAB }(n=100) \\
\text { CABG }(n=100)\end{array}$ & $\begin{array}{l}62.2 \pm 11.1 \\
62.5 \pm 9.45\end{array}$ & NR & $\begin{array}{l}41.3 \\
40.8\end{array}$ & $\begin{array}{l}3.39 \pm 1.04 \\
3.40 \pm 1.08\end{array}$ & Low \\
\hline Syed et $\mathrm{al}^{\mathrm{E} 88}$ & 2004 & Pulmonary gas exchange & $\begin{array}{l}\text { OPCAB }(\mathrm{n}=37) \\
\text { CABG }(\mathrm{n}=38)\end{array}$ & $\begin{array}{l}56.25(21-72) \ddagger \\
58.78(43-81) \ddagger\end{array}$ & NR & $\begin{array}{l}95 \\
87\end{array}$ & NR & Unclear \\
\hline Tang et $\mathrm{al}^{\mathrm{E} 89}$ & 2002 & $\begin{array}{l}\text { Kidney glomerular and } \\
\text { tubular injury }\end{array}$ & $\begin{array}{l}\text { OPCAB }(n=20) \\
\text { CABG }(n=20)\end{array}$ & $\begin{array}{l}64.8 \pm 6.9 \\
62.1 \pm 9.3\end{array}$ & NR & $\begin{array}{l}95 \\
90\end{array}$ & $\begin{array}{l}2.1 \pm 1.0 \\
2.5 \pm 0.7\end{array}$ & Unclear \\
\hline Tatoulis et $\mathrm{al}^{\mathrm{E} 90}$ & 2006 & $\begin{array}{l}\text { Systemic vascular } \\
\text { resistance at } 12 \mathrm{~h}\end{array}$ & $\begin{array}{l}\text { OPCAB }(\mathrm{n}=50) \\
\text { CABG }(\mathrm{n}=50)\end{array}$ & $\begin{array}{l}66 \pm 12 \\
64 \pm 11\end{array}$ & $\begin{array}{l}\text { LM } 8 \% \\
\text { LM } 8 \%\end{array}$ & NR & $\begin{array}{l}2.3 \pm 0.8 \\
2.9 \pm 0.9\end{array}$ & Low \\
\hline Tully et $\mathrm{al}^{\mathrm{E} 91}$ & 2008 & $\begin{array}{l}\text { Neuropsychologic } \\
\text { and quality-of-life } \\
\text { outcomes at } 6 \mathrm{mo}\end{array}$ & $\begin{array}{l}\text { OPCAB }(\mathrm{n}=30) \\
\text { CABG }(\mathrm{n}=36)\end{array}$ & $63.6 \pm 10.0$ & NR & $\begin{array}{l}96.7 \\
94.4\end{array}$ & $\begin{array}{l}2.23 \pm 0.52 \\
2.47 \pm 0.61\end{array}$ & Unclear \\
\hline Van Boven et $\mathrm{al}^{\mathrm{E} 92}$ & 2013 & $\begin{array}{l}\text { Protein } \mathrm{S} 100 \beta \\
\quad \text { concentration }\end{array}$ & $\begin{array}{l}\text { OPCAB }(n=10) \\
\text { CABG }(n=10)\end{array}$ & $\begin{array}{l}73.1 \pm 2.2 \\
73.3 \pm 1.4\end{array}$ & $\begin{array}{l}>50 \% \text { MV-CAD } \\
>50 \% \text { MV-CAD }\end{array}$ & $\begin{array}{l}50 \\
50\end{array}$ & $\begin{array}{l}3.6 \pm 0.8 \\
4.3 \pm 1.4\end{array}$ & Unclear \\
\hline Van Boven et $\mathrm{al}^{\mathrm{E} 93}$ & 2013 & $\begin{array}{l}\text { Periprocedural } \\
\text { inflammatory marker } \\
\text { release }\end{array}$ & $\begin{array}{l}\text { OPCAB }(n=20) \\
\text { CABG }(n=20)\end{array}$ & $\begin{array}{l}73.8 \pm 2.6 \\
74.6 \pm 4.0\end{array}$ & $\begin{array}{l}>50 \% \text { MV-CAD } \\
>50 \% \text { MV-CAD }\end{array}$ & $\begin{array}{l}100 \\
100\end{array}$ & $\begin{array}{l}3.8 \pm 0.8 \\
4.7 \pm 1.2\end{array}$ & Unclear \\
\hline Vedin et $\mathrm{al}^{\mathrm{E} 94}$ & 2006 & $\begin{array}{l}\text { Neurocognitive } \\
\text { function at } 6 \text { mo }\end{array}$ & $\begin{array}{l}\operatorname{OPCAB}(\mathrm{n}=33) \\
\operatorname{CABG}(\mathrm{n}=37)\end{array}$ & $\begin{array}{l}65(62-68) \ddagger \\
65(62-68) \ddagger\end{array}$ & $\begin{array}{l}>50 \% \text { MV-CAD; } \\
\text { LM } 0 \% \\
>50 \% \text { MV-CAD; } \\
\text { LM } 5 \%\end{array}$ & $\begin{array}{l}100 \\
95\end{array}$ & $\begin{array}{l}3(2-4) \ddagger \\
3(2-4) \ddagger\end{array}$ & Unclear \\
\hline Velissaris et $\mathrm{al}^{\mathrm{E95}}$ & 2003 & Gut mucosal oxygenation & $\begin{array}{l}\text { OPCAB }(\mathrm{n}=27) \\
\text { CABG }(\mathrm{n}=27)\end{array}$ & $\begin{array}{l}61.8 \pm 8.7 \\
63.1 \pm 8.5\end{array}$ & NR & $\begin{array}{l}88.9 \\
96.3\end{array}$ & $\begin{array}{l}2.5 \pm 0.8 \\
2.6 \pm 0.9\end{array}$ & Unclear \\
\hline Velissaris et $\mathrm{al}^{\mathrm{E} 96}$ & 2004 & Stress response & $\begin{array}{l}\text { OPCAB }(n=26) \\
\text { CABG }(n=26)\end{array}$ & $\begin{array}{l}61.3 \pm 8.5 \\
63.9 \pm 8.0\end{array}$ & NR & NR & $\begin{array}{l}2.4 \pm 0.9 \\
2.8 \pm 1.0\end{array}$ & Unclear \\
\hline Vural et $\mathrm{al}^{\mathrm{E} 97}$ & 1995 & $\begin{array}{l}\text { Periprocedural } \\
\text { hemodynamic } \\
\text { assessment }\end{array}$ & $\begin{array}{l}\text { OPCAB }(n=25) \\
\text { CABG }(n=25)\end{array}$ & $\begin{array}{l}47.2 \pm 11.3 \\
49.4 \pm 11.3\end{array}$ & $\begin{array}{l}>50 \% \text { SV-CAD } \\
>50 \% \text { SV-CAD }\end{array}$ & $\begin{array}{l}\text { NR } \\
\text { NR }\end{array}$ & $\begin{array}{l}1.12 \\
1.12\end{array}$ & Unclear \\
\hline
\end{tabular}


TABLE 1. Continued

\begin{tabular}{|c|c|c|c|c|c|c|c|c|}
\hline Study ${ }^{\text {reference }}$ & Year & Primary endpoint & $\begin{array}{c}\text { Randomization } \\
\text { (ITT) }\end{array}$ & $\begin{array}{c}\text { Age, } y, \\
\text { mean } \pm \text { SD }\end{array}$ & Extent of CAD & $\begin{array}{c}\text { IMA use, } \\
\%\end{array}$ & $\begin{array}{l}\text { No. of } \\
\text { grafts* }\end{array}$ & $\begin{array}{c}\text { Risk of } \\
\text { bias } \dagger\end{array}$ \\
\hline Wan et $\mathrm{al}^{\mathrm{E} 98}$ & 2004 & $\begin{array}{l}\text { Periprocedural } \\
\text { inflammatory } \\
\text { marker release }\end{array}$ & $\begin{array}{l}\text { OPCAB }(n=18) \\
\text { CABG }(n=19)\end{array}$ & $\begin{array}{l}63.61 \pm 10.5 \\
65.37 \pm 9.1\end{array}$ & $\begin{array}{l}>50 \% \mathrm{MV}-\mathrm{CAD} \\
>50 \% \mathrm{MV}-\mathrm{CAD}\end{array}$ & $\begin{array}{l}100 \\
100\end{array}$ & $\begin{array}{l}2.44 \pm 0.51 \\
2.79 \pm 0.54\end{array}$ & Unclear \\
\hline 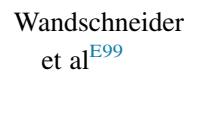 & 2000 & $\begin{array}{l}\text { Periprocedural } \\
\text { inflammatory } \\
\text { marker release }\end{array}$ & $\begin{array}{l}\text { OPCAB }(n=52) \\
\text { CABG }(n=67)\end{array}$ & $\begin{array}{l}65(38-82) \ddagger \\
66(48-78) \ddagger\end{array}$ & NR & $\begin{array}{l}65.6 \\
65.8\end{array}$ & $\begin{array}{l}2.34 \\
3.10\end{array}$ & Unclear \\
\hline Wehlin et $\mathrm{al}^{\mathrm{E} 100}$ & 2004 & $\begin{array}{l}\text { Periprocedural } \\
\text { inflammatory } \\
\text { marker release }\end{array}$ & $\begin{array}{l}\text { OPCAB }(n=22) \\
\text { CABG }(n=16)\end{array}$ & $66(61-70) \ddagger$ & $\begin{array}{l}\text { LM } 5 \% ;>50 \% \\
\text { MV-CAD } \\
\text { LM 12\%; }>50 \% \\
\text { MV-CAD }\end{array}$ & 100 & $\begin{array}{l}3(2-3) \ddagger \\
2.5(2-3) \ddagger\end{array}$ & Unclear \\
\hline Wehlin et al ${ }^{\mathrm{E} 101}$ & 2005 & $\begin{array}{l}\text { Peripheral blood } \\
\text { monocyte activation }\end{array}$ & $\begin{array}{l}\operatorname{OPCAB}(\mathrm{n}=11) \\
\operatorname{CABG}(\mathrm{n}=9)\end{array}$ & $\begin{array}{l}64(58-70) \ddagger \\
67(59-74) \ddagger\end{array}$ & $\begin{array}{l}\text { LM } 0 \% ;>50 \% \\
\text { MV-CAD } \\
\text { LM } 11 \% ;>50 \% \\
\text { MV-CAD }\end{array}$ & NR & $\begin{array}{l}2(2-3) \ddagger \\
2(2-3) \ddagger\end{array}$ & Unclear \\
\hline Yu et al ${ }^{\mathrm{E} 102}$ & 2014 & Early clinical outcomes & $\begin{array}{l}\text { OPCAB }(\mathrm{n}=51) \\
\text { CABG }(\mathrm{n}=51)\end{array}$ & $\begin{array}{l}63.7 \pm 8.6 \\
65.7 \pm 7.6\end{array}$ & $\begin{array}{l}\text { LM } 27.5 \% \\
\text { LM } 31.4 \%\end{array}$ & $\begin{array}{l}100 \\
100\end{array}$ & $\begin{array}{l}3.02 \pm 0.55 \\
3.14 \pm 0.53\end{array}$ & Low \\
\hline Zamvar et al $^{\mathrm{E} 103}$ & 2002 & $\begin{array}{l}\text { Neurocognitive function } \\
\text { at } 10 \mathrm{wk}\end{array}$ & $\begin{array}{l}\operatorname{OPCAB}(\mathrm{n}=30) \\
\operatorname{CABG}(\mathrm{n}=30)\end{array}$ & $\begin{array}{l}63.5 \pm 9.1 \\
61.6 \pm 10\end{array}$ & $\begin{array}{l}\text { LM } 13.3 \% ;>50 \% \\
\text { MV-CAD } \\
\text { LM } 6.6 \% ;>50 \% \\
\text { MV-CAD }\end{array}$ & 100 & $\begin{array}{l}2.93 \pm 0.37 \\
2.93 \pm 0.45\end{array}$ & Low \\
\hline
\end{tabular}

$I T T$, Intention-to-treat; $S D$, standard deviation; $C A D$, coronary artery disease; $I M A$, internal mammary artery; $H R Q O L$, health-related quality of life; $O P C A B$, off-pump coronary artery bypass; $C A B G$, coronary artery bypass grafting; $M V$ - $C A D$, multivessel coronary artery disease; $L M$, left main; $N R$, not reported; $B B S$, best bypass surgery trial; $M A C C E$, major adverse cardiac or cerebrovascular accident; $M I$, myocardial infarction, $L C O S$, low cardiac output syndrome; BHACAS 1 , beating heart against cardioplegic arrest study 1 ; BHACAS 2, eating heart against cardioplegic arrest study 2; NA, not available; CORONARY, CABG off or on pump revascularization study; ICU, intensive care unit; CRISP, The Coronary artery bypass grafting in high-RISk patients randomized to off- or on-Pump surgery; DOORS, Danish on-pump versus off-pump randomization study; STEMI, ST-segment elevation MI; $S V$-CAD; single vessel coronary artery disease; GOPCABE; German off-pump coronary artery bypass grafting in elderly patients; $c T n$, cardiac troponin; JOCRI, Japanese off-pump coronary revascularization investigation study; $C H F$, chronic heart failure; $T V R$, target vessel revascularization; $A F$, atrial fibrillation/flutter; $C P K-M B$, creatine phosphokinase-muscle brain; MASS III, off-pump and on-pump stable multivessel coronary artery bypass grafting; Octopus, comparison of on-pump and offpump CORONARY bypass surgery in low-risk patients; $A R D S$, acute respiratory distress syndrome; $M C P$ - 1 , monocyte chemo-attractant protein; $V E G F$, vascular endothelial growth factor; PAI-1, plasminogen activator inhibitor-1; $L V E F$, left ventricle ejection fraction; TF, tissue factor; PRAGUE-4, A randomized comparison between off-pump and on-pump surgery PRAGUE-4; PRAGUE-6, Off-pump versus on-pump coronary artery bypass graft surgery in patients with EuroSCORE $\geq 6$; $P R A G U E-11$, A randomized comparison between off-pump and on-pump surgery PRAGUE-11; PROMISS, The prospective randomized comparison of off-pump and on-pump multivessel coronary artery bypass surgery; ROOBY, veterans affairs randomized on/off bypass study; SMART, surgical management of arterial revascularization therapies. *Reported as in included studies. $\dagger$ Risk of bias according to Cochrane criteria; a study was considered at high risk of bias if $\geq 2$ of the components were at high risk. $\ddagger$ Reported as range. $\S$ Reported for separate arms.

respect to $\leq 30$-day all-cause mortality and MI. Finally, a significant linear relationship was found between risk profile and benefits of OPCAB for all endpoints: all-cause mortality, MI, and cerebral stroke. The robustness and stability of the results were confirmed in sensitivity analyses.

The current 2014 European Society of Cardiology guidelines on myocardial revascularization assign class IIa level B recommendations for performing OPCAB in high-risk patients in high-volume off-pump centers. ${ }^{25}$ The 2011 American College of Cardiology/American Association for Thoracic Surgery guidelines recommend OPCAB in patients with renal impairment, and suggest considering beating heart revascularization to reduce perioperative bleeding and the need for transfusions. ${ }^{26}$

Various randomized studies have failed to show significant differences in the perioperative stroke rate between surgery with and without $\mathrm{ECC},{ }^{27,28}$ and both European and American guidelines point to conflicting evidence regarding the role of off-pump surgery in providing a better neurologic outcome. This apparent contradiction with the available registries data ${ }^{29,30}$ reporting a significantly decreased incidence of stroke with OPCAB may be explained by the fact that cerebral stroke represents a relatively rare entity after CABG, and thus even the largest randomized studies are underpowered to prove a possible advantage of one technique over the other. Indeed, the CORONARY trial, the largest trial to date, found no difference in the incidence of stroke in the 2 groups at 30 days $(1.0 \%$ for OPCAB vs $1.1 \%$ for $\mathrm{CABG})$. Furthermore, although 3 previous meta-analyses of RTCs demonstrated significant $(30 \%$ $50 \%$ ) reductions in the risk of stroke, ${ }^{31-33}$ a systematic review of RCTs called these outcomes into question, showing no clear benefit of OPCAB in this regard $(O R$, $0.76 ; 95 \%$ CI, 0.54-1.06). ${ }^{34}$

Possible causes of perioperative cerebral stroke include postoperative atrial fibrillation in the hypercoagulable state and thromboembolic events in the setting of cerebral artery disease, particularly in female patients with a previous 


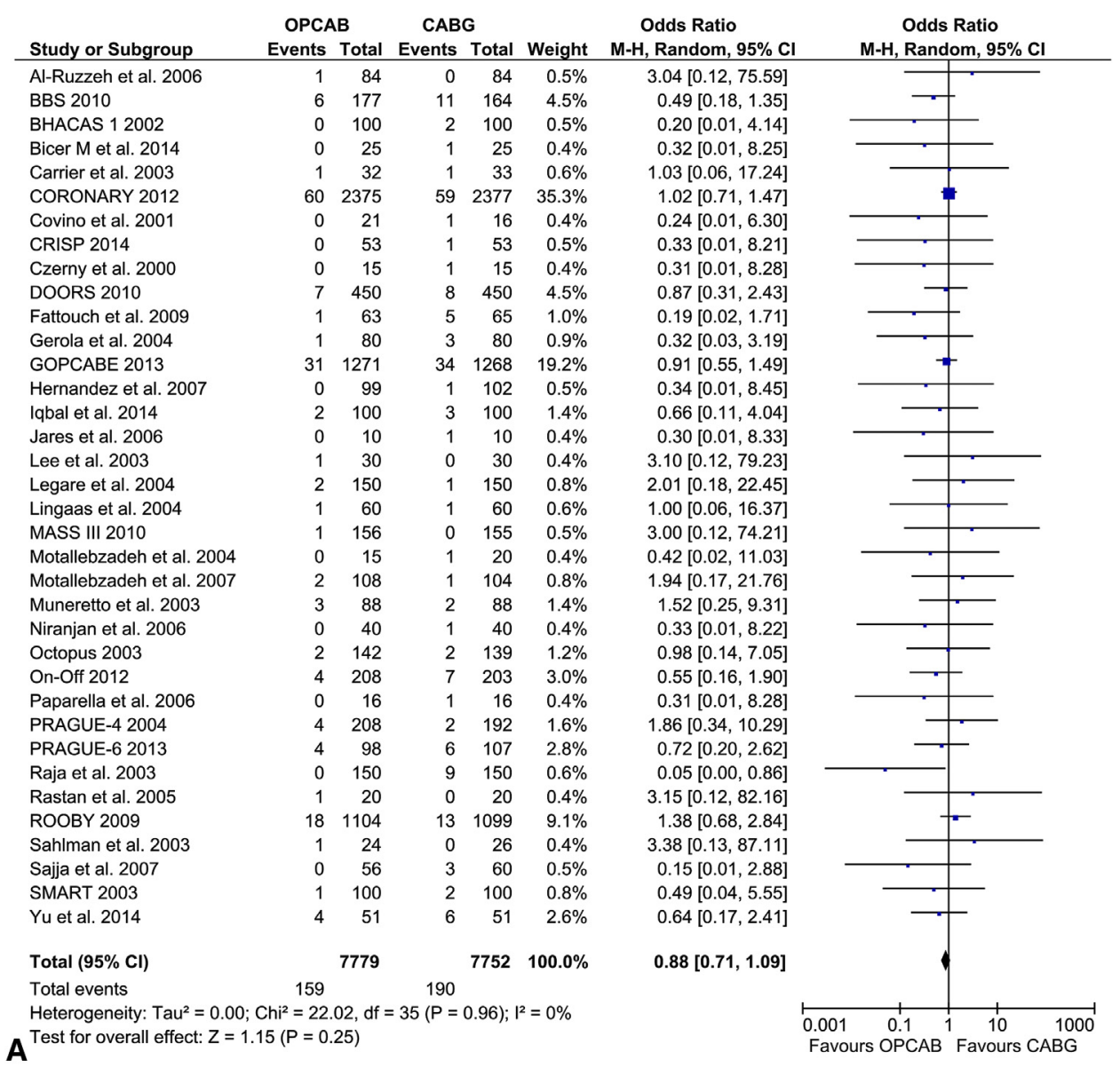

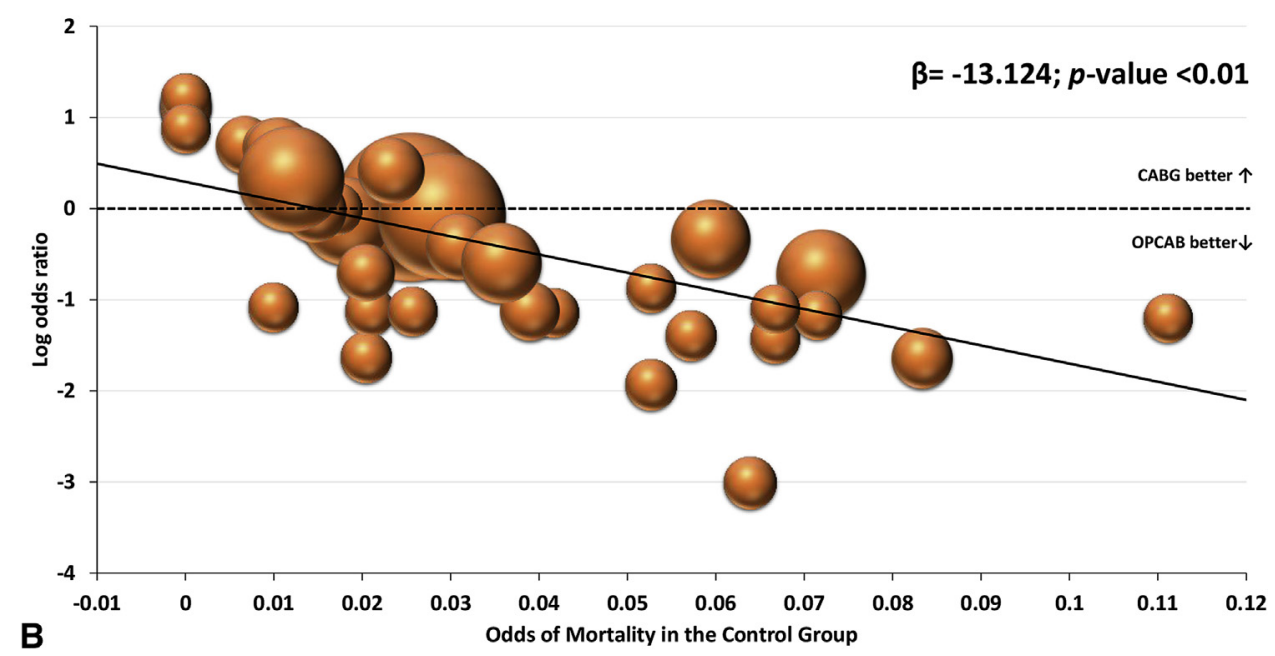

FIGURE 2. A, Pooled ORs and $95 \%$ CIs for $\leq 30$-day all-cause mortality. Size of squares is proportional to the statistical weight of each trial. Diamonds represent OR point estimates; horizontal lines represent $95 \%$ CIs for given point estimates. The positioning of both diamonds and squares (along with $95 \%$ CIs) beyond the vertical line (unit value) suggests a significant outcome. B, Weighted random-effects meta-regression analysis regressing the log OR of all-cause mortality against the control group all-cause mortality expressed as odds. Negative values of the log OR ( $y$-axis) mean more benefits in all-cause mortality associated with OPCAB, whereas the mortality rate of the control group, CABG ( $x$-axis), represents the risk profile of the patient population included in each trial. The size of the circle corresponds to the inverse variance of the log-OR, and thus is related to the statistical weight of the individual trial. $O P C A B$, Off-pump coronary artery bypass; $C A B G$, coronary artery bypass grafting; $M$ - $H$, Mantel-Haenszel; $C I$, confidence interval; $d f$, degrees of freedom. 


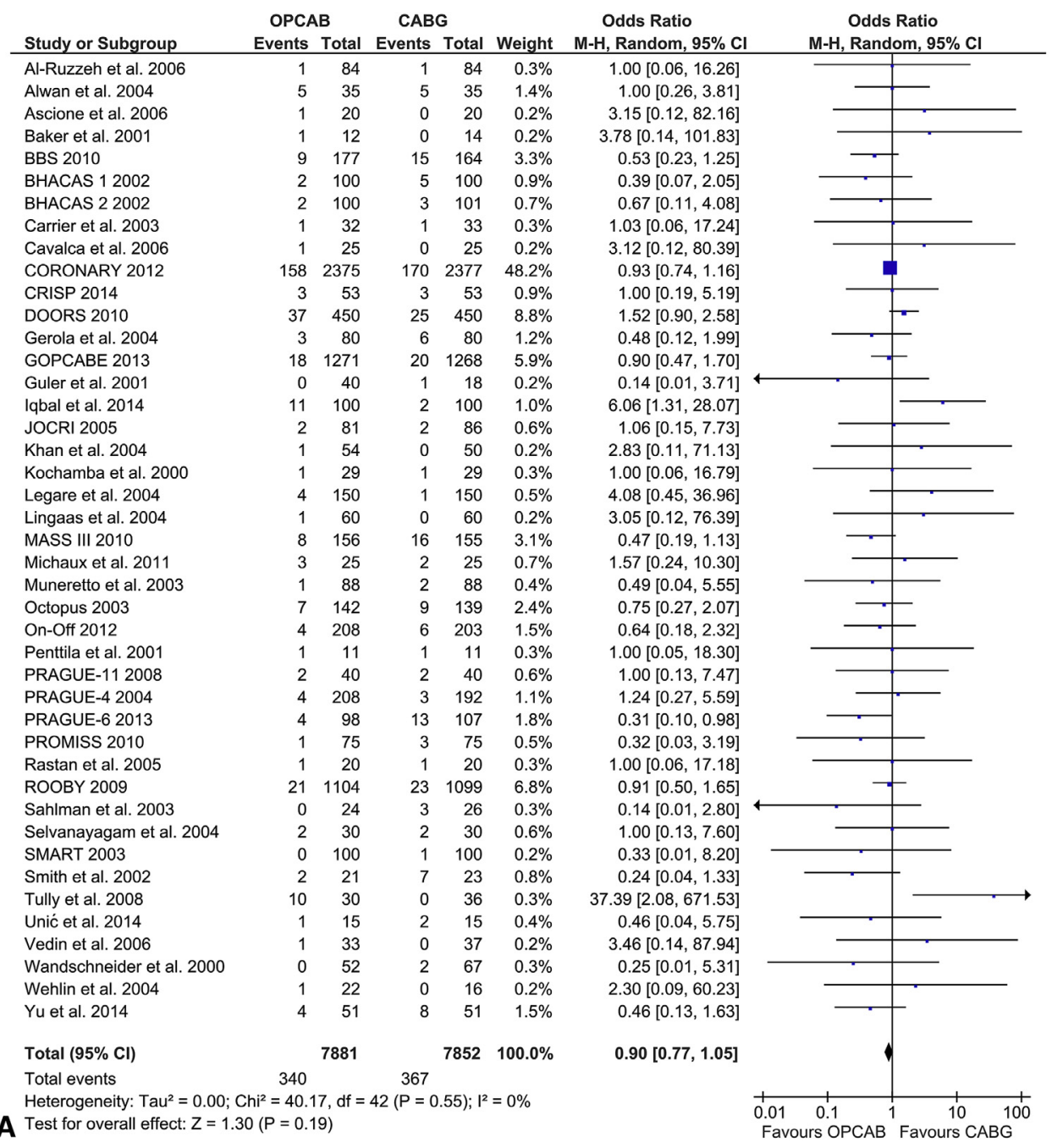

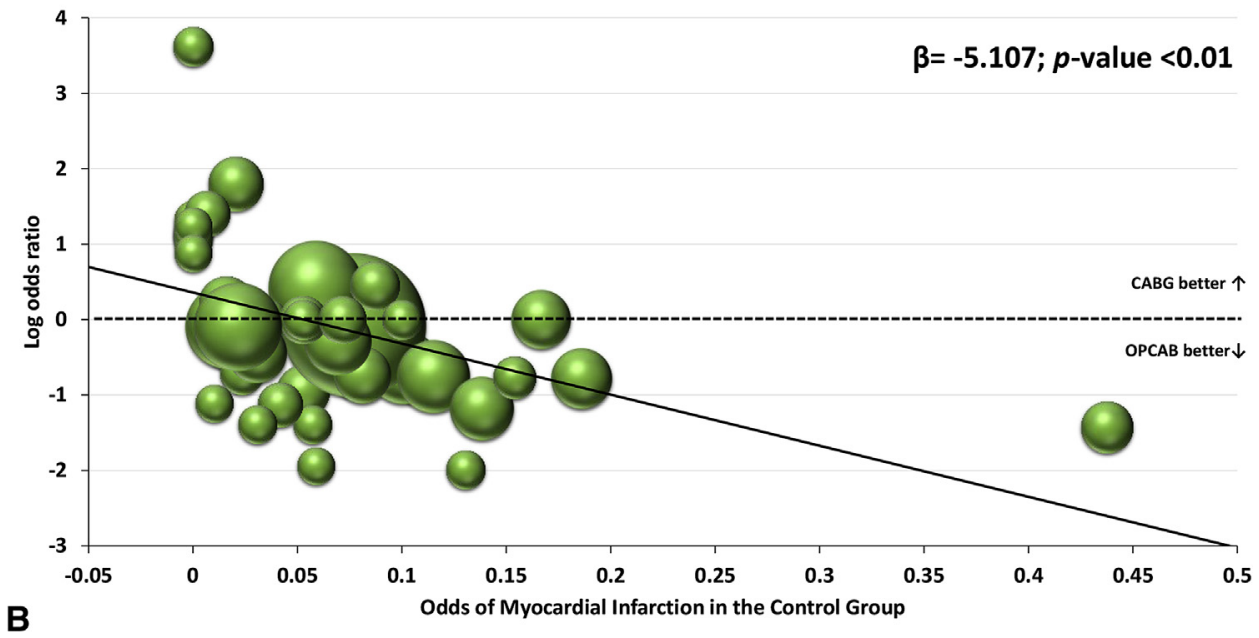

FIGURE 3. A, Pooled ORs and $95 \%$ CIs for $\leq 30$-day MI. Size of squares is proportional to the statistical weight of each trial. Diamonds represent OR point estimates; horizontal lines represent $95 \%$ CIs for given point estimates. The positioning of both diamonds and squares (along with $95 \%$ CIs) beyond the vertical line (unit value) suggests a significant outcome. B, Weighted random-effects meta-regression analysis regressing the log OR of MI against the incidence of MI in the control group expressed as odds. Negative values of the log OR ( $y$-axis) mean more benefits for MI associated with OPCAB, whereas the MI rate in the control group, CABG ( $x$-axis), represents the risk profile of the patient population included in each trial. The size of the circle corresponds to the inverse variance of the log-OR, and thus is related to the statistical weight of the individual trial. $O P C A B$, Off-pump coronary artery bypass; $C A B G$, coronary artery bypass grafting; $M-H$, Mantel-Haenszel; $C I$, confidence interval; $d f$, degrees of freedom. 


\begin{tabular}{|c|c|c|c|c|c|c|c|c|}
\hline Study or Subgroup & \multicolumn{2}{|c|}{ OPCAB } & \multicolumn{2}{|c|}{ CABG } & Weight & $\begin{array}{c}\text { Odds Ratio } \\
\text { M-H, Random, } 95 \% \mathrm{Cl}\end{array}$ & \multicolumn{2}{|c|}{$\begin{array}{c}\text { Odds Ratio } \\
\text { M-H, Random, } 95 \% \mathrm{Cl}\end{array}$} \\
\hline Al-Ruzzeh et al. 2006 & 2 & 84 & 2 & 84 & $1.6 \%$ & $1.00[0.14,7.27]$ & & \\
\hline BBS 2010 & 7 & 177 & 6 & 164 & $5.0 \%$ & $1.08[0.36,3.30]$ & & \\
\hline BHACAS 12002 & 2 & 100 & 3 & 100 & $1.9 \%$ & $0.66[0.11,4.04]$ & & \\
\hline BHACAS 22002 & 1 & 100 & 3 & 101 & $1.2 \%$ & $0.33[0.03,3.23]$ & & \\
\hline Bicer M et al. 2014 & 0 & 25 & 3 & 25 & $0.7 \%$ & $0.13[0.01,2.58]$ & & \\
\hline Carrier et al. 2003 & 0 & 32 & 1 & 33 & $0.6 \%$ & $0.33[0.01,8.49]$ & & \\
\hline CORONARY 2012 & 24 & 2375 & 27 & 2377 & $20.4 \%$ & $0.89[0.51,1.54]$ & & - \\
\hline CRISP 2014 & 2 & 53 & 1 & 53 & $1.1 \%$ & $2.04[0.18,23.19]$ & & \\
\hline Diegeler et al. 2000 & 0 & 20 & 1 & 20 & $0.6 \%$ & $0.32[0.01,8.26]$ & & \\
\hline DOORS 2010 & 10 & 450 & 18 & 450 & $10.1 \%$ & $0.55[0.25,1.20]$ & & \\
\hline GOPCABE 2013 & 26 & 1271 & 32 & 1268 & $22.7 \%$ & $0.81[0.48,1.36]$ & & - \\
\hline Hernandez et al. 2007 & 0 & 99 & 3 & 102 & $0.7 \%$ & $0.14[0.01,2.80]$ & & \\
\hline Iqbal et al. 2014 & 2 & 100 & 6 & 100 & $2.4 \%$ & $0.32[0.06,1.62]$ & & - \\
\hline Jares et al. 2006 & 0 & 10 & 1 & 10 & $0.6 \%$ & $0.30[0.01,8.33]$ & & \\
\hline JOCRI 2005 & 0 & 81 & 1 & 86 & $0.6 \%$ & $0.35[0.01,8.71]$ & & \\
\hline Jongman et al. 2014 & 0 & 30 & 1 & 30 & $0.6 \%$ & $0.32[0.01,8.24]$ & & \\
\hline Kok et al. 2014 & 0 & 30 & 1 & 30 & $0.6 \%$ & $0.32[0.01,8.24]$ & & \\
\hline Lee et al. 2003 & 0 & 30 & 1 & 30 & $0.6 \%$ & $0.32[0.01,8.24]$ & & \\
\hline Legare et al. 2004 & 2 & 150 & 0 & 150 & $0.7 \%$ & $5.07[0.24,106.45]$ & & \\
\hline Lingaas et al. 2004 & 0 & 60 & 2 & 60 & $0.7 \%$ & $0.19[0.01,4.11]$ & & \\
\hline Lund et al. 2003 & 0 & 29 & 1 & 23 & $0.6 \%$ & $0.25[0.01,6.54]$ & & \\
\hline MASS III 2010 & 2 & 156 & 4 & 155 & $2.1 \%$ & $0.49[0.09,2.72]$ & & \\
\hline Medved et al. 2008 & 1 & 30 & 2 & 30 & $1.0 \%$ & $0.48[0.04,5.63]$ & & \\
\hline Michaux et al. 2011 & 1 & 25 & 0 & 25 & $0.6 \%$ & $3.12[0.12,80.39]$ & & \\
\hline Motallebzadeh et al. 2004 & 0 & 15 & 1 & 20 & $0.6 \%$ & $0.42[0.02,11.03]$ & & \\
\hline Motallebzadeh et al. 2007 & 1 & 108 & 3 & 104 & $1.2 \%$ & $0.31[0.03,3.07]$ & & \\
\hline Muneretto et al. 2003 & 0 & 88 & 2 & 88 & $0.7 \%$ & $0.20[0.01,4.13]$ & & \\
\hline Nesher et al. 2006 & 2 & 61 & 3 & 64 & $1.9 \%$ & $0.69[0.11,4.27]$ & & \\
\hline Niranjan et al. 2006 & 1 & 40 & 1 & 40 & $0.8 \%$ & $1.00[0.06,16.56]$ & & \\
\hline Octopus 2003 & 1 & 142 & 2 & 139 & $1.1 \%$ & $0.49[0.04,5.42]$ & & \\
\hline On-Off 2012 & 0 & 208 & 1 & 203 & $0.6 \%$ & $0.32[0.01,7.99]$ & & \\
\hline PRAGUE-4 2004 & 0 & 208 & 2 & 192 & $0.7 \%$ & $0.18[0.01,3.83]$ & & \\
\hline PRAGUE-6 2013 & 2 & 98 & 3 & 107 & $1.9 \%$ & $0.72[0.12,4.42]$ & & \\
\hline PROMISS 2010 & 0 & 75 & 2 & 75 & $0.7 \%$ & $0.19[0.01,4.12]$ & & \\
\hline ROOBY 2009 & 14 & 1104 & 8 & 1099 & $8.2 \%$ & $1.75[0.73,4.19]$ & & \\
\hline Sahlman et al. 2003 & 1 & 24 & 1 & 26 & $0.8 \%$ & $1.09[0.06,18.40]$ & & \\
\hline Sajja et al. 2007 & 0 & 56 & 1 & 60 & $0.6 \%$ & $0.35[0.01,8.80]$ & & \\
\hline Selvanayagam et al. 2004 & 0 & 30 & 1 & 30 & $0.6 \%$ & $0.32[0.01,8.24]$ & & \\
\hline SMART 2003 & 2 & 100 & 2 & 100 & $1.6 \%$ & $1.00[0.14,7.24]$ & & \\
\hline Yu et al. 2014 & 0 & 51 & 5 & 51 & $0.7 \%$ & $0.08[0.00,1.53]$ & & - \\
\hline Total $(95 \% \mathrm{Cl})$ & & 7925 & & 7904 & $100.0 \%$ & $0.72[0.56,0.92]$ & $\checkmark$ & \\
\hline Total events & 106 & & 158 & & & & & \\
\hline $\begin{array}{l}\text { Heterogeneity: } \mathrm{Tau}^{2}=0.00 \\
\text { Test for overall effect: } Z=\end{array}$ & $\begin{array}{l}\mathrm{Chi}^{2}=21 . \\
2(\mathrm{P}=0 . \mathrm{C}\end{array}$ & $\begin{array}{l}81, \mathrm{df}= \\
.009)\end{array}$ & $=39(\mathrm{P}=$ & 0.99); । & $\left.\right|^{2}=0 \%$ & & $\begin{array}{l}0.010 .1 \\
\text { Favours OPCAB }\end{array}$ & $\begin{array}{l}110 \\
\text { Favours } \mathrm{C}\end{array}$ \\
\hline
\end{tabular}

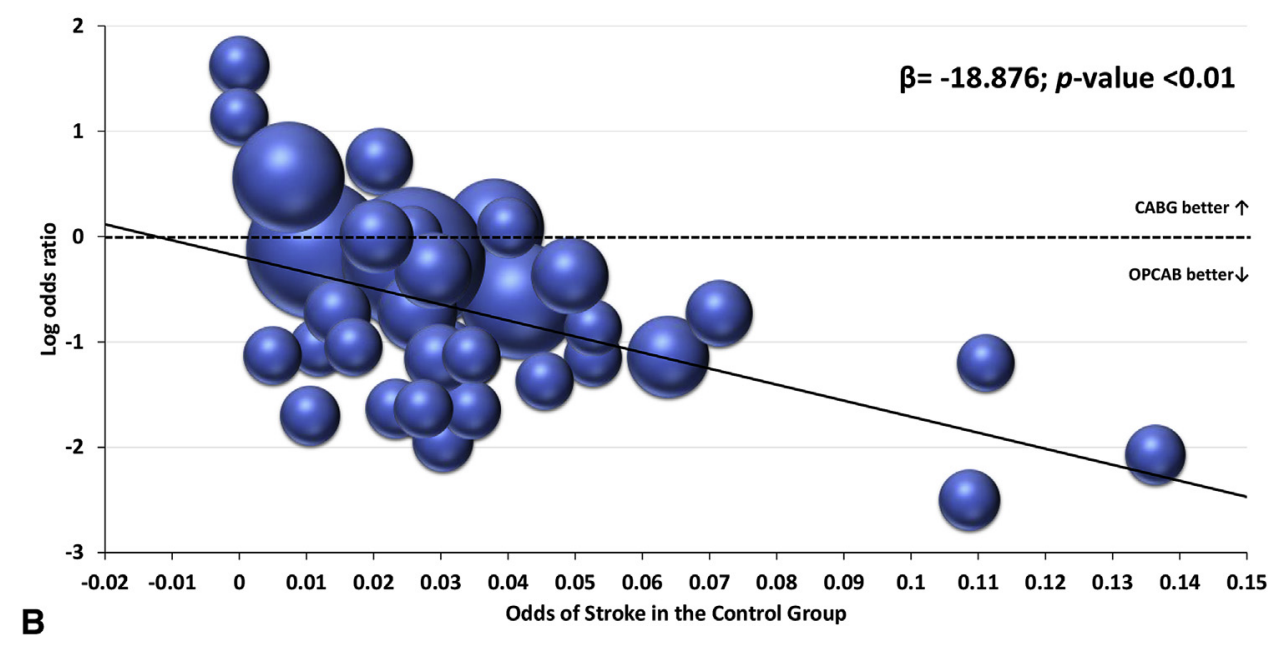

FIGURE 4. A, Pooled ORs and $95 \%$ CIs for $\leq 30$-day cerebral stroke. Size of squares is proportional to the statistical weight of each trial. Diamonds represent OR point estimates; horizontal lines represent $95 \%$ CIs for given point estimate. The positioning of both diamonds and squares (along with $95 \% \mathrm{CIs}$ ) beyond the vertical line (unit value) suggests a significant outcome. B, Weighted random-effects meta-regression analysis regressing the log OR of cerebral stroke against the incidence of cerebral stroke in the control group expressed as odds. Negative values of the log OR ( $y$-axis) mean more benefits in cerebral stroke associated with OPCAB, whereas the cerebral stroke rate of the control group, CABG ( $x$-axis), represents the risk profile of the patient population included in each trial. The size of the circle corresponds to the inverse variance of the log-OR, and thus is related to the statistical weight of the individual trial. OPCAB, Off-pump coronary artery bypass; $C A B G$, coronary artery bypass grafting; $M$ - $H$, Mantel-Haenszel; $C I$, confidence interval; $d f$, degrees of freedom. 


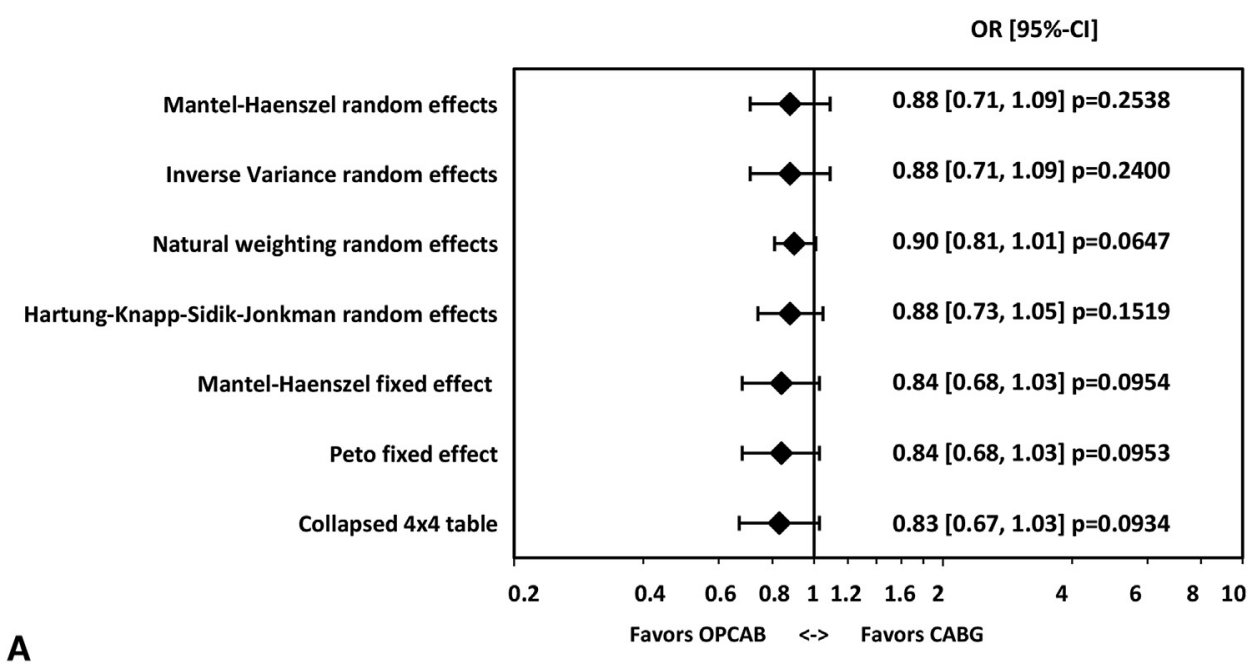

A

OR [95\%-Cl]

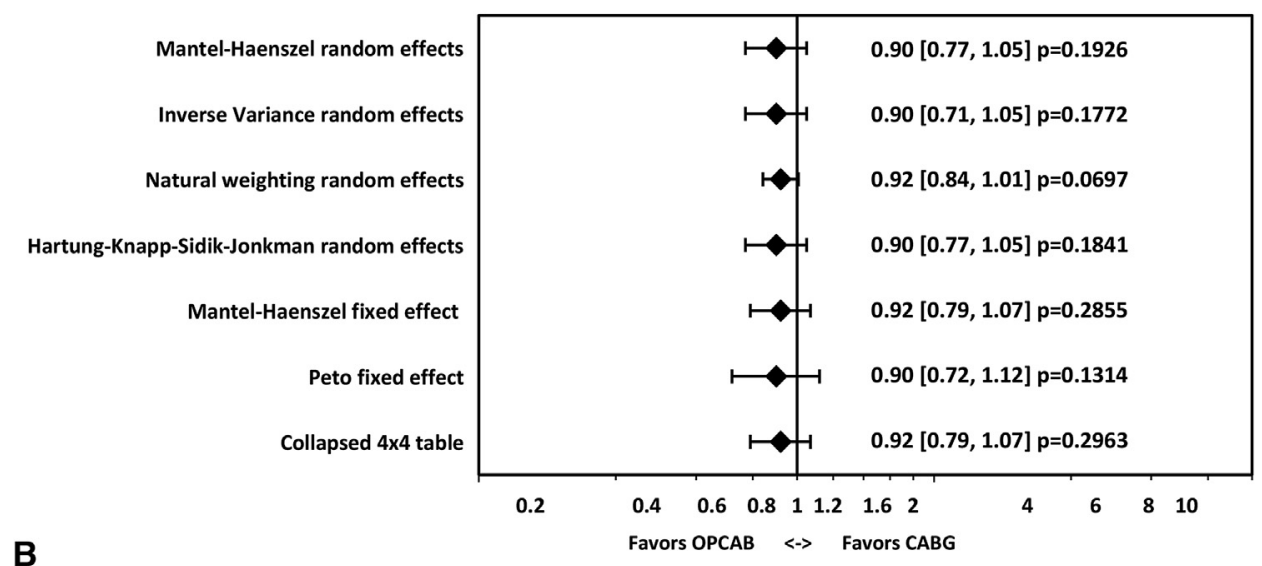

OR [95\%-Cl]

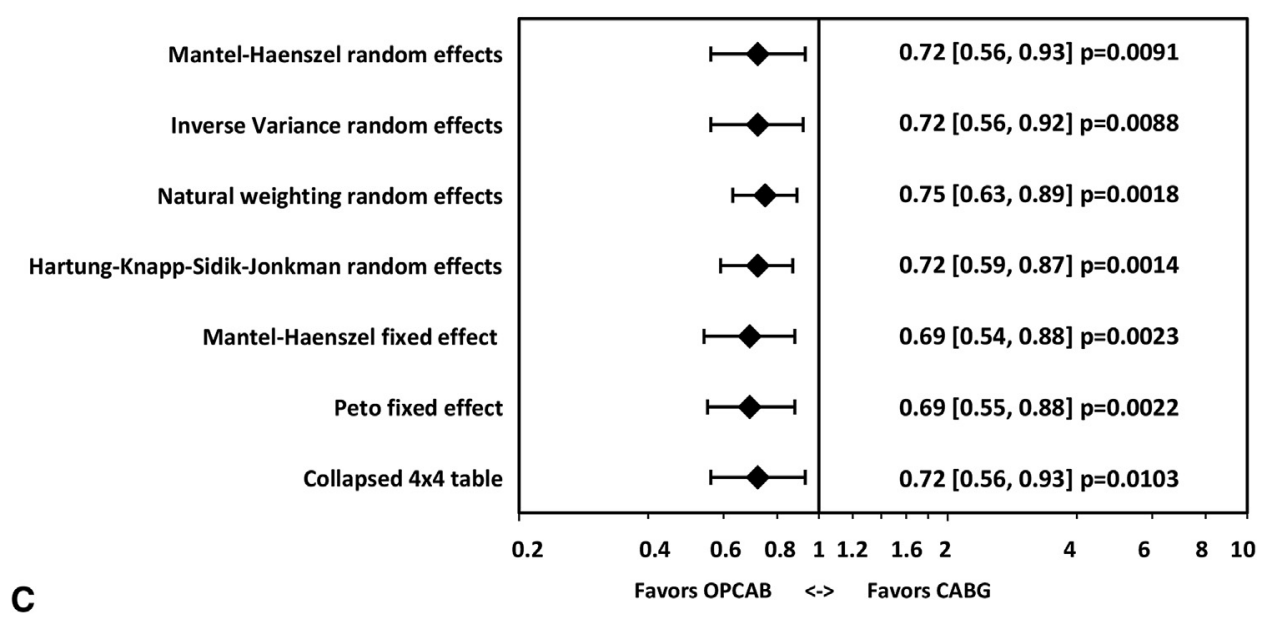

FIGURE 5. Sensitivity analysis of estimated pooled ORs and $95 \%$ CIs using 7 different approaches: Mantel-Haenszel random effects (as in the main analysis), inverse variance (DerSimonian-Laird) method, natural weighting, Hartung-Knapp-Sidik Jonkman, Mantel-Haenszel fixed-effect, Peto method, and a collapsed $4 \times 4$ table for $\leq 30$-day all-cause mortality (A), MI (B), and cerebral stroke (C). $C I$, Confidence interval; $O P C A B$, off-pump coronary artery bypass; $C A B G$, coronary artery bypass grafting; $O R$, odds ratio 
history of cerebral stroke. It is the aortic manipulation and consequent embolization of atherosclerotic material that play crucial roles in the occurrence of cerebral stroke in patients undergoing $\mathrm{CABG}$, however. ${ }^{35}$ OPCAB confers the benefit of avoiding CPB; it eliminates the need for aortic cannulation and cross-clamping and thereby minimizes the risk of neck and brain vessel embolism. Performing the proximal anastomoses during cross-clamping is a possible strategy for reducing further manipulation of the aorta during conventional CABG; however, the application of a side-biting clamp is usually described, which poses an increased risk of dislodging fragile material from the aortic wall. OPCAB presents the same technical issue in proximal anastomoses; however, especially in the presence of a diffusely diseased aorta, off-pump procedures via complete in situ grafting (no-touch; double internal mammary artery and/or T- or Y-grafting) preclude any type of clamping and may reduce the risk of postoperative neurologic events and cognitive function impairment. ${ }^{36,37}$ In studies included in the current meta-analysis, the proportion of the no-touch technique was seldom reported. The benefits in terms of postoperative stroke are expected to increase with an increasing number of procedures performed without aortic manipulation. Indeed, in a recent meta-analysis of 7 observational studies, clampless OPCAB was associated with significantly reduced risk of stroke compared with conventional CABG $(0.38 \%$ vs $1.87 \%$; risk ratio, $0.27 ; 95 \% \mathrm{CI}, 0.14-0.58 ; P<.001)$ and with OPCAB using a partial clamp $(0.31 \%$ vs $1.35 \%$; risk ratio, $0.34 ; 95 \%$ CI, $0.18-0.65 ; P=.001) .{ }^{34}$ Another study comparing clampless $\mathrm{OPCAB}$ with conventional $\mathrm{CABG}$ found a $56 \%$ and $44 \%$ significant reduction of mortality and composite endpoint death and stroke, respectively, with the former approach ${ }^{38}$ In the present meta-analysis, which included the most recently published RCTs, a significant $28 \%$ reduction in the odds of stroke was associated with OPCAB.

Possibly that most important finding of our present analysis is the significant linear relationship between patient risk profile and the beneficial effects of OPCAB with respect to the single endpoints all-cause mortality, MI, and stroke. Observational studies have suggested that OPCAB is particularly beneficial in high-risk patients, including those with left ventricular dysfunction, high calcific load, age $>75$ years, diabetes, renal failure, left main stem disease, reoperations, chronic pulmonary disease, and an overall European System for Cardiac Operative Risk Evaluation (EuroSCORE) score of $>5$. In a recent analysis of 14,766 patients, ${ }^{39}$ OCPAB was associated with mortality benefit in the higher risk quartiles (38\% and $55 \%$ reduction in the odds of mortality in the 3rd and 4th quartiles, respectively, as defined by the Society of Thoracic Surgeons Predicted Risk of Mortality Score). Of note, there was no difference in operative mortality between
OPCAB and conventional $\mathrm{CABG}$ for patients in the lower 2 risk quartiles. Although that analysis was limited by its nonrandomized design, a review of the database revealed that patients receiving $\mathrm{OPCAB}$ were older and sicker than those undergoing conventional $\mathrm{CABG}$ and had a significantly greater predicted risk of mortality and numerous morbidities. Our results, using solely randomized data, confirm what has long been reported in the large registries. ${ }^{40,41}$

A common fundamental criticism of the majority of previous randomized studies was that they tend to invariably enroll selected very-low-risk to low-risk patients. In this setting, with adverse events rates of $\sim 1 \%-2 \%$, demonstration of benefits with either technique in a single trial would require enormous study groups. Through risk profile meta-regression, ${ }^{42}$ combining study-level aggregate data of included studies, we were able to demonstrate a significant relationship between the benefits of OPCAB and baseline patient risk profiles as expressed by the odds of a given outcome in the control group (CABG).

Why high-risk patients are less likely to develop complications after OPCAB than their conventional CABG counterparts remains a subject of ongoing debate. The use of CPB has been associated with a systemic inflammatory response affecting multiple organ systems, including the brain, heart, lungs, kidney, and the gastrointestinal tract. Avoidance of CPB and, in turn, interactions between the inflammatory, coagulation, and fibrinolytic cascades confer complex, organ-specific benefits, especially in high-risk patients. Yokoyama et $\mathrm{al}^{43}$ reported reduced rates of neurologic, renal, coagulopathy, and respiratory complications in $\mathrm{OPCAB}$ recipients compared with CABG controls. In particular, $9.3 \%$ of the patients with chronic obstructive pulmonary disease undergoing conventional $\mathrm{CABG}$ required prolonged postoperative mechanical ventilation, whereas no patient in the OPCAB group experienced delayed extubation. This finding points for the first time to the negative effects of fluid shifts and inflammatory reaction secondary to CPB in a compromised respiratory system and surely merits further research. These organ-specific benefits translated finally in no operative mortality for $\mathrm{OPCAB}$, whereas a postoperative mortality ranging from $2.3 \%$ to $8.3 \%$ was reported in CPB groups.

The findings on mortality benefit with OPCAB in high risk patients corroborate on a larger scale the hypothesis that beyond an individual patient's risk threshold, OPCAB performs better than CABG. Al-Ruzzeh et $\mathrm{al}^{10}$ reported that high-risk patients (preoperative EuroSCORE $\geq 5$ ) with multivessel disease showed significant reductions in the incidence of perioperative $\mathrm{MI}$ and other major complications $(P=.008)$, reduced length of intensive care unit stay, and lower mortality after OPCAB surgery compared with conventional $\mathrm{CPB}$ myocardial revascularization. 
Since 1999, the EuroSCORE has evolved from "additive" and "logistic" to EuroSCORE II owing to changing clinical pictures of the cardiac surgery patients. In a meta-analysis published in 2009 (8 studies, 24,673 subjects), additive and logistic EuroSCORE algorithms performed similarly, and cumulative evidence suggested comparable performance in $\mathrm{CABG}$ and $\mathrm{OPCAB}$ procedures (area under the curve, 0.767 for CABG and 7.66 for OPCAB) ${ }^{43}$ Both risk models significantly overestimated mortality, however. In contrast, EuroSCORE II, which was introduced into clinical practice from initial experience, calculates the risk, which is statistically significantly lower than the risk as assessed by the EuroSCORE, with a reduction of approximately $50 \%$, although definitive data are not yet available. ${ }^{44}$ This decreased calculated risk for a surgical procedure will certainly have clinical consequences for the decision to perform an operation in high-risk patients. The results of the present study may in fact provide guidance for surgeons in performing $\mathrm{OPCAB}$ in this patient population or, alternatively, to refer such patients to high-volume centers experienced in off-pump techniques. Although this needs to be confirmed in a large comparative study, surgeons who prefer a selective approach could use EuroSCORE II for the decision making and patient selection for OPCAB.

Several limitations of our meta-analysis should be acknowledged. First, the analysis shares the limitations of original studies included; thus, results were analyzed on the trial level and not on the patient level. Although detailed sensitivity analyses were undertaken, given the heterogeneity of the study protocols, clinically relevant differences could have been missed and might have been better assessed in a meta-analysis of individual patient data. The criteria for patient inclusion were broad across the studies, more closely reflecting current clinical practice, enrolling both high-risk patients and patients undergoing elective surgery. A subtle underlying bias of the trials included remains a possible limitation of this and any systematic review. We accounted for bias by excluding studies at high risk in the sensitivity analysis. Event rates observed in the present analysis, and particularly mortality, were slightly higher than those reported in the available registries $(2.25 \%$ vs $2.07 \%$ as reported in the Society of Thoracic Surgeons database). ${ }^{45}$ In addition, there could be additional confounders not accounted for in the analysis, such as surgeon experience; one influential study included in the present meta-analysis ${ }^{8}$ was indeed criticized because CABG was performed by surgical trainees under the supervision of attending surgeons, who were remarkably inexperienced in the off-pump procedure and much more experienced in the on-pump procedure. The 53 participating surgeons enrolled on average only 8 patients per year over a 5 -year period and had unacceptably high rates of conversion to on-pump surgery $(12 \%)$ and incomplete revascularization $(18 \%)$. We acknowledge the need for larger, higher-quality studies addressing training and surgeon experience in $\mathrm{OPCAB}$, proper case selection for $\mathrm{OPCAB}$, timing and effect of conversion to on-pump surgery, and impact of the degree of revascularization (complete vs noncomplete).

On the other hand, because our main analysis was performed using a random-effects model, the present study accounts for baseline between- and within-study variations and is the first to demonstrate, on a comprehensive level, a mortality benefit with OPCAB in patients at higher risk. The findings on significant mortality riskprofile meta-regression are further corroborated in the analysis of MI and stroke.

\section{CONCLUSIONS}

OPCAB is associated with a significant $(28 \%)$ reduction in the odds of $\leq 30$-day stroke compared with conventional $\mathrm{CABG}$, but there is no difference with respect to all-cause mortality and MI between the 2 techniques. A significant linear relationship between benefits in all-cause mortality, $\mathrm{MI}$, and stroke with $\mathrm{OPCAB}$ and patient risk profile was shown, suggesting that $\mathrm{OPCAB}$ should be seriously considered in high-risk patients.

\section{Conflict of Interest Statement}

Dr Navarese has received honoraria for lectures from Eli Lilly. All other authors have nothing to disclose with regard to commercial support.

\section{References}

1. O'Connor CM, Velazquez EJ, Gardner LH, Smith PK, Newman MF, Landolfo KP, et al. Comparison of coronary artery bypass grafting versus medical therapy on long-term outcome in patients with ischemic cardiomyopathy (a 25-year experience from the Duke Cardiovascular Disease Databank). Am J Cardiol. 2002;90:101-7.

2. Sipahi I, Akay MH, Dagdelen S, Blitz A, Alhan C. Coronary artery bypass grafting vs percutaneous coronary intervention and long-term mortality and morbidity in multivessel disease: meta-analysis of randomized clinical trials of the arterial grafting and stenting era. JAMA Intern Med. 2014;174:223-30.

3. Serruys PW, Morice MC, Kappetein AP, Colombo A, Holmes DR, Mack MJ, et al. Percutaneous coronary intervention versus coronary-artery bypass grafting for severe coronary artery disease. N Engl J Med. 2009;360:961-72.

4. Hart JC, Puskas JD, Sabik JF III. Off-pump coronary revascularization: current state of the art. Semin Thorac Cardiovasc Surg. 2002;14:70-81.

5. Meharwal ZS, Mishra YK, Kohli V, Bapna R, Singh S, Trehan N. Off-pump multivessel coronary artery surgery in high-risk patients. Ann Thorac Surg. 2002; 74:S1353-7.

6. Marui A, Okabayashi H, Komiya T, Tanaka S, Furukawa Y, Kita T, et al. Benefits of off-pump coronary artery bypass grafting in high-risk patients. Circulation. 2012;126:S151-7.

7. Sharony R, Bizekis CS, Kanchuger M, Galloway AC, Saunders PC, Applebaum R, et al. Off-pump coronary artery bypass grafting reduces mortality and stroke in patients with atheromatous aortas: a case control study. Circulation. 2003;108(Suppl 1):II15-20.

8. Shroyer AL, Grover FL, Hattler B, Collins JF, McDonald GO, Kozora E, et al. On-pump versus off-pump coronary-artery bypass surgery. $N$ Engl J Med. 2009;361:1827-37.

9. Lamy A, Devereaux PJ, Prabhakaran D, Taggart DP, Hu S, Paolasso E, et al. Off-pump or on-pump coronary-artery bypass grafting at 30 days. $N$ Engl $J$ Med. 2012;366:1489-97. 
10. Al-Ruzzeh S, Nakamura K, Athanasiou T, Modine T, George S, Yacoub M, et al. Does off-pump coronary artery bypass (OPCAB) surgery improve the outcome in high-risk patients? A comparative study of 1398 high-risk patients. Eur J Cardiothorac Surg. 2003;23:50-5.

11. Raja SG. Myocardial revascularization for the elderly: current options, role of off-pump coronary artery bypass grafting and outcomes. Curr Cardiol Rev. 2012;8:26-36.

12. Shamseer L, Moher D, Clarke M, Ghersi D, Liberati A, Petticrew M, et al. Preferred reporting items for systematic review and meta-analysis protocols (PRISMA-P) 2015: elaboration and explanation. BMJ. 2015; 349:g7647.

13. Higgins JP, Altman DG, Gotzsche PC, Jüni P, Moher D, Oxman AD, et al. The Cochrane Collaboration's tool for assessing risk of bias in randomised trials. BMJ. 2011;343:d5928.

14. Cohen J. Weighted kappa: nominal scale agreement with provision for scaled disagreement or partial credit. Psychol Bull. 1968;70:213-20.

15. Light DW. Effectiveness and efficiency under competition: the Cochrane test. BMJ. 1991;303:1253-4.

16. Higgins JPT, Green S, eds. Cochrane handbook for systematic reviews of interventions, version 5.1.0. Available at: www.cochrane-handbook.org/. Accessed February 1, 2015.

17. Kuss O, Gummert JF, Börgermann J. Meta-analyses with rare events should use adequate methods. J Thorac Cardiovasc Surg. 2008;136:241.

18. Shuster JJ. Empirical vs natural weighting in random effects meta-analysis. Stat Med. 2010;29:1259-65.

19. IntHout J, Ioannidis JP, Borm GF. The Hartung-Knapp-Sidik-Jonkman method for random effects meta-analysis is straightforward and considerably outperforms the standard DerSimonian-Laird method. BMC Med Res Methodol. 2014; $14: 25$.

20. Yusuf S, Peto R, Lewis J, Collins R, Sleight P. Beta blockade during and after myocardial infarction: an overview of the randomized trials. Prog Cardiovasc Dis. 1985;27:335-71.

21. Bradburn MJ, Deeks JJ, Berlin JA, Russell Localio A. Much ado about nothing: a comparison of the performance of meta-analytical methods with rare events. Stat Med. 2007;26:53-77.

22. Wilson E, Underwood M, Puckrin, O, Letto K, Doyle R, Caravan H, et al. The arcsine transformation: has the time come for retirement? Available at: http:// www.mun.ca/biology/dschneider/b7932/B7932Final10Dec2010.pdf. Accessed February 1, 2015.

23. Sterne JA, Sutton AJ, Ioannidis JP, Terrin N, Jones DR, Lau J, et al. Recommendations for examining and interpreting funnel plot asymmetry in meta-analyses of randomised controlled trials. BMJ. 2011;343:d4002.

24. Stijnen T, Hamza TH, Ozdemir P. Random effects meta-analysis of event outcome in the framework of the generalized linear mixed model with applications in sparse data. Stat Med. 2010;29:3046-67.

25. Windecker S, Kolh P, Alfonso F, Collet JP, Cremer J, Falk V, et al. 2014 ESC/EACTS guidelines on myocardial revascularization: the Task force on Myocardial Revascularization of the European Society of Cardiology (ESC) and the European Association for Cardio-Thoracic Surgery (EACTS), developed with the special contribution of the European Association of Percutaneous Cardiovascular Interventions (EAPCI). Eur Heart J. 2014;35: 2541-619.

26. Hillis LD, Smith PK, Anderson JL, Bittl JA, Bridges CR, Byrne JG, et al. 2011 ACCF/AHA guideline for coronary artery bypass graft surgery: executive summary: a report of the American College of Cardiology Foundation/American Heart Association Task Force on Practice Guidelines. Circulation. 2011;124: 2610-42.

27. Moller CH, Perko MJ, Lund JT, Andersen LW, Kelbaek H, Madsen JK, et al. No major differences in 30-day outcomes in high-risk patients randomized to off-pump versus on-pump coronary bypass surgery: the best bypass surgery trial. Circulation. 2010;121:498-504.

28. Al-Ruzzeh S, George S, Bustami M, Wray J, Ilsley C, Athanasiou T, et al. Effect of off-pump coronary artery bypass surgery on clinical, angiographic, neurocognitive, and quality of life outcomes: randomised controlled trial BMJ. 2006:332:1365.

29. Hannan EL, Wu C, Smith CR, Higgins RS, Carlson RE, Culliford AT, et al Off-pump versus on-pump coronary artery bypass graft surgery: differences in short-term outcomes and in long-term mortality and need for subsequent revascularization. Circulation. 2007;116:1145-52.

30. Puskas JD, Kilgo PD, Lattouf OM, Thourani VH, Cooper WA, Vassiliades TA, et al. Off-pump coronary bypass provides reduced mortality and morbidity and equivalent 10-year survival. Ann Thorac Surg. 2008;86:1139-46.

31. Sedrakyan A, Wu AW, Parashar A, Bass EB, Treasure T. Off-pump surgery is associated with reduced occurrence of stroke and other morbidity as compared with traditional coronary artery bypass grafting: a meta-analysis of systematically reviewed trials. Stroke. 2006;37:2759-69.

32. Afilalo J, Rasti M, Ohayon SM, Shimony A, Eisenberg MJ. Off-pump vs. on-pump coronary artery bypass surgery: an updated meta-analysis and meta-regression of randomized trials. Eur Heart J. 2012;33:1257-67.

33. Palmerini T, Biondi-Zoccai G, Riva DD, Mariani A, Savini C, Di Eusanio M, et al Risk of stroke with percutaneous coronary intervention compared with on-pump and off-pump coronary artery bypass graft surgery: evidence from a comprehensive network meta-analysis. Am Heart J. 2013;165:910-7.e14.

34. Moller CH, Penninga L, Wetterslev J, Steinbruchel DA, Gluud C. Off-pump versus on-pump coronary artery bypass grafting for ischaemic heart disease. Cochrane Database Syst Rev. 2012:3:CD007224.

35. Kapetanakis EI, Stamou SC, Dullum MK, Hill PC, Haile E, Boyce SW, et al The impact of aortic manipulation on neurologic outcomes after coronary artery bypass surgery: a risk-adjusted study. Ann Thorac Surg. 2004;78:1564-71.

36. Moss E, Puskas JD, Thourani VH, Kilgo P, Chen EP, Leshnower BG, et al Avoiding aortic clamping during coronary artery bypass grafting reduces postoperative stroke. J Thorac Cardiovasc Surg. 2015;149:175-80.

37. Szwed K, Pawliszak W, Anisimowicz L, Bucinski A, Borkowska A. Short-term outcome of attention and executive functions from aorta no-touch and traditional off-pump coronary artery bypass surgery. World J Biol Psychiatry. 2014;15: $397-403$.

38. Edelman JJ, Yan TD, Bannon PG, Wilson MK, Vallely MP. Coronary artery bypass grafting with and without manipulation of the ascending aorta: a meta-analysis. Heart Lung Circ. 2011;20:318-24.

39. Börgermann J, Hakim K, Renner A, Parsa A, Aboud A, Becker T, et al Clampless off-pump versus conventional coronary artery revascularization: a propensity score analysis of 788 patients. Circulation. 2012;126(11 Suppl 1):S176-82.

40. Puskas JD, Thourani VH, Kilgo P, Cooper W, Vassiliades T, Vega JD, et al Off-pump coronary artery bypass disproportionately benefits high-risk patients. Ann Thorac Surg. 2009;88:1142-7.

41. Kuss O, von Salviati B, Börgermann J. Off-pump versus on-pump coronary artery bypass grafting: a systematic review and meta-analysis of propensity score analyses. J Thorac Cardiovasc Surg. 2010;140:829-35.

42. De Luca G, Navarese E, Marino P. Risk profile and benefits from Gp IIb-IIIa inhibitors among patients with ST-segment elevation myocardial infarction treated with primary angioplasty: a meta-regression analysis of randomized trials. Eur Heart J. 2009;30:2705-13.

43. Yokoyama T, Baumgartner FJ, Gheissari A, Capouya ER, Panagiotides GP, Declusin RJ. Off-pump versus on-pump coronary bypass in high-risk subgroups. Ann Thorac Surg. 2000;70:1546-50.

44. Parolari A, Pesce LL, Trezzi M, Loardi C, Kassem S, Brambillasca C, et al Performance of euroSCORE in CABG and off-pump coronary artery bypass grafting: single institution experience and meta-analysis. Eur Heart J. 2009; 30:297-304.

45. Weintraub WS, Grau-Sepulveda MV, Weiss JM, O'Brien SM, Peterson ED Kolm P, et al. Comparative effectiveness of revascularization strategies. $N$ Engl J Med. 2012;366:1467-76.

Key Words: coronary disease, cardiopulmonary bypass, cerebrovascular disorders 


\section{References to studies included in the meta-analysis:}

E1. Al-Ruzzeh S, George S, Bustami M, Wray J, Ilsley C, Athanasiou T, et al. Effect of off-pump coronary artery bypass surgery on clinical, angiographic, neurocognitive, and quality of life outcomes: randomised controlled trial. BMJ. 2006;332:1365.

E2. Alwan K, Falcoz PE, Alwan J, Mouawad W, Oujaimi G, Chocron S, et al. Beating versus arrested heart coronary revascularization: evaluation by cardiac troponin I release. Ann Thorac Surg. 2004;77:2051-5.

E3. Ascione R, Ghosh A, Reeves BC, Arnold J, Potts M, Shah A, et al. Retinal and cerebral microembolization during coronary artery bypass surgery: a randomized, controlled trial. Circulation. 2005;112:3833-8.

E4. Ascione R, Talpahewa S, Rajakaruna C, Reeves BC, Lovell AT, Cohen A, et al. Splanchnic organ injury during coronary surgery with or without cardiopulmonary bypass: a randomized, controlled trial. Ann Thorac Surg. 2006;81:97-103.

E5. Baker RA, Andrew MJ, Ross IK, Knight JL. The Octopus II stabilizing system: biochemical and neuropsychological outcomes in coronary artery bypass surgery. Heart Surg Forum. 2001;4(Suppl 1):S19-23.

E6. Moller CH, Perko MJ, Lund JT, Andersen LW, Kelbaek H, Madsen JK, et al. No major differences in 30-day outcomes in high-risk patients randomized to off-pump versus on-pump coronary bypass surgery: the best bypass surgery trial. Circulation. 2010;121:498-504.

E7. Angelini GD, Taylor FC, Reeves BC, Ascione R. Early and midterm outcome after off-pump and on-pump surgery in Beating Heart Against Cardioplegic Arrest Studies (BHACAS 1 and 2): a pooled analysis of two randomised controlled trials. Lancet. 2002;359:1194-9.

E8. Ascione R, Caputo M, Calori G, Lloyd CT, Underwood MJ, Angelini GD. Predictors of atrial fibrillation after conventional and beating heart coronary surgery: a prospective, randomized study. Circulation. 2000;102:1530-5.

E9. Bicer M, Senturk T, Yanar M, Tutuncu A, Oral AY, Ulukaya E, et al. Effects of off-pump versus on-pump coronary artery bypass grafting: apoptosis, inflammation, and oxidative stress. Heart Surg Forum. 2014;17:E271-6.

E10. Blacher C, Neumann J, Jung LA, Lucchese FA, Ribeiro JP. Off-pump coronary artery bypass grafting does not reduce lymphocyte activation. Int $\mathrm{J}$ Cardiol. 2005;101:473-9.

E11. Bonacchi M, Prifti E, Maiani M, Bartolozzi F, Di Eusanio M, Leacche M. Does off-pump coronary revascularization reduce the release of the cerebral markers, S-100beta and NSE? Heart Lung Circ. 2006;15:314-9.

E12. Caputo M, Yeatman M, Narayan P, Marchetto G, Ascione R, Reeves BC, et al. Effect of off-pump coronary surgery with right ventricular assist device on organ function and inflammatory response: a randomized controlled trial. Ann Thorac Surg. 2002;74:2088-95.

E13. Carrier M, Perrault LP, Jeanmart H, Martineau R, Cartier R, Page P. Randomized trial comparing off-pump to on-pump coronary artery bypass grafting in high-risk patients. Heart Surg Forum. 2003;6:E89-92.

E14. Cavalca V, Sisillo E, Veglia F, Tremoli E, Cighetti G, Salvi L, et al. Isoprostanes and oxidative stress in off-pump and on-pump coronary bypass surgery. Ann Thorac Surg. 2006;81:562-7.

E15. Chowdhury UK, Malik V, Yadav R, Seth S, Ramakrishnan L, Kalaivani M, et al. Myocardial injury in coronary artery bypass grafting: on-pump versus off-pump comparison by measuring high-sensitivity C-reactive protein, cardiac troponin I, heart-type fatty acid-binding protein, creatine kinaseMB, and myoglobin release. J Thorac Cardiovasc Surg. 2008;135:1110-9.

E16. Lamy A, Devereaux PJ, Prabhakaran D, Taggart DP, Hu S, Paolasso E, et al. Off-pump or on-pump coronary-artery bypass grafting at 30 days. $N$ Engl J Med. 2012;366:1489-97.

E17. Covino E, Santise G, Di Lello F, De Amicis V, Bonifazi R, Bellino I, et al. Surgical myocardial revascularization $(\mathrm{CABG})$ in patients with pulmonary disease: beating heart versus cardiopulmonary bypass. J Cardiovasc Surg (Torino). 2001;42:23-6.

E18. Rogers CA, Pike K, Campbell H, Reeves BC, Angelini GD, Gray A, et al. Coronary artery bypass grafting in high-RISk patients randomised to off- or on-pump surgery: a randomised controlled trial (the CRISP trial). Health Technol Assess. 2014;18. v-xx,1-157.

E19. Czerny M, Baumer H, Kilo J, Lassnigg A, Hamwi A, Vukovich T, et al. Inflammatory response and myocardial injury following coronary artery bypass grafting with or without cardiopulmonary bypass. Eur J Cardiothorac Surg. 2000;17:737-42.

E20. Czerny M, Baumer H, Kilo J, Zuckermann A, Grubhofer G, Chevtchik O, et al. Complete revascularization in coronary artery bypass grafting with and without cardiopulmonary bypass. Ann Thorac Surg. 2001;71:165-9.
E21. Diegeler A, Hirsch R, Schneider F, Schilling LO, Falk V, Rauch T, et al. Neuromonitoring and neurocognitive outcome in off-pump versus conventional coronary bypass operation. Ann Thorac Surg. 2000;69:1162-6.

E22. Dorman BH, Kratz JM, Multani MM, Baron R, Farrar E, Walton S, et al. A prospective, randomized study of endothelin and postoperative recovery in off-pump versus conventional coronary artery bypass surgery. J Cardiothorac Vasc Anesth. 2004;18:25-9.

E23. Houlind K, Kjeldsen BJ, Madsen SN, Rasmussen BS, Holme SJ, Nielsen PH, et al. On-pump versus off-pump coronary artery bypass surgery in elderly patients: results from the Danish on-pump versus off-pump randomization study. Circulation. 2012;125:2431-9.

E24. Fattouch K, Guccione F, Dioguardi P, Sampognaro R, Corrado E, Caruso M, et al. Off-pump versus on-pump myocardial revascularization in patients with ST-segment elevation myocardial infarction: a randomized trial. J Thorac Cardiovasc Surg. 2009;137:650-6.

E25. Formica F, Mariani S, Broccolo F, Caruso R, Corti F, D’Alessandro S, et al. Systemic and myocardial inflammatory response in coronary artery bypass graft surgery with miniaturized extracorporeal circulation: differences with a standard circuit and off-pump technique in a randomized clinical trial. ASAIO J. 2013;59:600-6.

E26. Gasz B, Benkö L, Jancsó G, Lantos J, Szántó Z, Alotti N, et al. Comparison of inflammatory response following coronary revascularization with or without cardiopulmonary bypass. Exp Clin Cardiol. 2004;9:26-30.

E27. Gasz B, Lenard L, Benko L, Borsiczky B, Szanto Z, Lantos J, et al. Expression of CD97 and adhesion molecules on circulating leukocytes in patients undergoing coronary artery bypass surgery. Eur Surg Res. 2005;37: 281-9.

E28. Gerola LR, Buffolo E, Jasbik W, Botelho B, Bosco J, Brasil LA, et al. Off-pump versus on-pump myocardial revascularization in low-risk patients with one or two vessel disease: perioperative results in a multicenter randomized controlled trial. Ann Thorac Surg. 2004;77:569-73.

E29. Gu YJ, Mariani MA, van Oeveren W, Grandjean JG, Boonstra PW. Reduction of the inflammatory response in patients undergoing minimally invasive coronary artery bypass grafting. Ann Thorac Surg. 1998;65:420-4.

E30. Güler M, Kirali K, Toker ME, Bozbuga N, Omeroglu SN, Akinci E, et al. Different CABG methods in patients with chronic obstructive pulmonary disease. Ann Thorac Surg. 2001;71:152-7.

E31. Gönenc A, Hacisevki A, Bakkaloglu B, Soyagir A, Torun M, Karagöz H, et al. Oxidative stress is decreased in off-pump versus on-pump coronary artery surgery. J Biochem Mol Biol. 2006;39:377-82.

E32. Diegeler A, Börgermann J, Kappert U, Breuer M, Böning A, Ursulescu A, et al. Off-pump versus on-pump coronary-artery bypass grafting in elderly patients. N Engl J Med. 2013;368:1189-98.

E33. Gulielmos V, Menschikowski M, Dill H, Eller M, Thiele S, Tugtekin SM, et al. Interleukin-1, interleukin-6 and myocardial enzyme response after coronary artery bypass grafting - a prospective randomized comparison of the conventional and three minimally invasive surgical techniques. Eur $J$ Cardiothorac Surg. 2000;18:594-601.

E34. Hernandez F Jr, Brown JR, Likosky DS, Clough RA, Hess AL, Roth RM, et al. Neurocognitive outcomes of off-pump versus on-pump coronary artery bypass: a prospective randomized controlled trial. Ann Thorac Surg. 2007; 84:1897-903.

E35. Hoel TN, Videm V, Mollnes TE, Saatvedt K, Brosstad F, Fiane AE, et al. Off-pump cardiac surgery abolishes complement activation. Perfusion. 2007;22:251-6.

E36. Iqbal J, Ghaffar A, Shahbaz A, Abid AR. Stroke after coronary artery bypass surgery with and without cardiopulmonary bypass. J Ayub Med Coll Abbottabad. 2014;26:123-8

E37. Jares M, Vanek T, Bednar F, Maly M, Snircova J, Straka Z. Off-pump versus on-pump coronary artery surgery. Int Heart J. 2007;48:57-67.

E38. Kobayashi J, Tashiro T, Ochi M, Yaku H, Watanabe G, Satoh T, et al. Early outcome of a randomized comparison of off-pump and on-pump multiple arterial coronary revascularization. Circulation. 2005;112:I338-43.

E39. Johansson-Synnergren M, Nilsson F, Bengtsson A, Jeppsson A, Wiklund L. Off-pump CABG reduces complement activation but does not significantly affect peripheral endothelial function: a prospective randomized study. Scand Cardiovasc J. 2004;38:53-8.

E40. Jongman RM, Zijlstra JG, Kok WF, van Harten AE, Mariani MA, Moser J, et al. Off-pump CABG surgery reduces systemic inflammation compared with on-pump surgery but does not change systemic endothelial responses: a prospective randomized study. Shock. 2014;42:121-8. 
E41. Khan NE, De Souza A, Mister R, Flather M, Clague J, Davies S, et al. A randomized comparison of off-pump and on-pump multivessel coronaryartery bypass surgery. $N$ Engl J Med. 2004;350:21-8.

E42. Kochamba GS, Yun KL, Pfeffer TA, Sintek CF, Khonsari S. Pulmonary abnormalities after coronary arterial bypass grafting operation: cardiopulmonary bypass versus mechanical stabilization. Ann Thorac Surg. 2000; 69:1466-70.

E43. Kok WF, van Harten AE, Koene BM, Mariani MA, Koerts J, Tucha O, et al. A pilot study of cerebral tissue oxygenation and postoperative cognitive dysfunction among patients undergoing coronary artery bypass grafting randomised to surgery with or without cardiopulmonary bypass. Anaesthesia. 2014;69:613-22.

E44. Krejca M, Skiba J, Szmagala P, Gburek T, Bochenek A. Cardiac troponin T release during coronary surgery using intermittent cross-clamp with fibrillation, on-pump and off-pump beating heart. Eur J Cardiothorac Surg. 1999;16:337-41.

E45. Kunes P, Lonsky V, Mandak J, Kolackova M, Andrys C, Kudlova M, et al. The long pentraxin 3 in cardiac surgery: distinct responses in "on-pump" and "off-pump" patients. Scand Cardiovasc J. 2007;41:171-9.

E46. Lee JD, Lee SJ, Tsushima WT, Yamauchi H, Lau WT, Popper J, et al. Benefits of off-pump bypass on neurologic and clinical morbidity: a prospective randomized trial. Ann Thorac Surg. 2003;76:18-25.

E47. Légaré JF, Buth KJ, King S, Wood J, Sullivan JA, Hancock Friesen C, et al. Coronary bypass surgery performed off pump does not result in lower in-hospital morbidity than coronary artery bypass grafting performed on pump. Circulation. 2004;109:887-92.

E48. Lingaas PS, Hol PK, Lundblad R, Rein KA, Tonnesen TI, Svennevig JL, et al. Clinical and angiographic outcome of coronary surgery with and without cardiopulmonary bypass: a prospective randomized trial. Heart Surg Forum. 2004; 7:37-41.

E49. Lingaas PS, Hol PK, Lundblad R, Rein KA, Mathisen L, Smith HJ, et al. Clinical and radiologic outcome of off-pump coronary surgery at 12 months follow-up: a prospective randomized trial. Ann Thorac Surg. 2006;81: 2089-95.

E50. Lloyd CT, Ascione R, Underwood MJ, Gardner F, Black A, Angelini GD. Serum S-100 protein release and neuropsychologic outcome during coronary revascularization on the beating heart: a prospective randomized study. $J$ Thorac Cardiovasc Surg. 2000;119:148-54.

E51. Lund C, Hol PK, Lundblad R, Fosse E, Sundet K, Tennoe B, et al. Comparison of cerebral embolization during off-pump and on-pump coronary artery bypass surgery. Ann Thorac Surg. 2003;76:765-70.

E52. Lund C, Sundet K, Tennoe B, Hol PK, Rein KA, Fosse E, et al. Cerebral ischemic injury and cognitive impairment after off-pump and on-pump coronary artery bypass grafting surgery. Ann Thorac Surg. 2005;80:2126-31.

E53. Malik V, Kale SC, Chowdhury UK, Ramakrishnan L, Chauhan S, Kiran U. Myocardial injury in coronary artery bypass grafting: on-pump versus off-pump comparison by measuring heart-type fatty-acid-binding protein release. Tex Heart Inst J. 2006;33:321-7.

E54. Mandak J, Pojar M, Cibicek N, Lonsky V, Palicka V, Kakrdova D, et al. Impact of cardiopulmonary bypass on peripheral tissue metabolism and microvascular blood flow. Perfusion. 2008;23:339-46.

E55. Matata BM, Sosnowski AW, Galiñanes M. Off-pump bypass graft operation significantly reduces oxidative stress and inflammation. Ann Thorac Surg. 2000;69:785-91.

E56. Hueb W, Lopes NH, Pereira AC, Hueb AC, Soares PR, Favarato D, et al. Five-year follow-up of a randomized comparison between off-pump and on-pump stable multivessel coronary artery bypass grafting. The MASS III Trial. Circulation. 2010;122(11 Suppl):S48-52.

E57. Medved I, Anic D, Zrnic B, Ostric M, Saftic I. Off-pump versus on-pump-intermittent aortic cross clamping-myocardial revascularisation: single center experience. Coll Antropol. 2008;32:381-4.

E58. Michaux I, Filipovic M, Skarvan K, Schneiter S, Schumann R, Zerkowski HR, et al. Effects of on-pump versus off-pump coronary artery bypass graft surgery on right ventricular function. J Thorac Cardiovasc Surg. 2006;131: 1281-8.

E59. Michaux I, Filipovic M, Skarvan K, Bolliger D, Schumann R, Bernet F, et al. A randomized comparison of right ventricular function after on-pump versus off-pump coronary artery bypass graft surgery. J Thorac Cardiovasc Surg. 2011;141:361-7.

E60. Modine T, Zannis C, Salleron J, Provot F, Gourlay T, Duhamel A, et al. A prospective randomized study to evaluate the renal impact of surgical revascularization strategy in diabetic patients. Interact Cardiovasc Thorac Surg. 2010;11:406-10.

E61. Motallebzadeh R, Bland JM, Markus HS, Kaski JC, Jahangiri M Neurocognitive function and cerebral emboli: randomized study of on-pump versus off-pump coronary artery bypass surgery. Ann Thorac Surg. 2007;83:475-82.

E62. Motallebzadeh R, Kanagasabay R, Bland M, Kaski JC, Jahangiri M. S100 protein and its relation to cerebral microemboli in on-pump and off-pump coronary artery bypass surgery. Eur J Cardiothorac Surg. 2004;25:409-14.

E63. Muneretto C, Bisleri G, Negri A, Manfredi J, Metra M, Nodari S, et al Off-pump coronary artery bypass surgery technique for total arterial myocardial revascularization: a prospective randomized study. Ann Thorac Surg. 2003;76:778-82.

E64. Nesher N, Frolkis I, Vardi M, Sheinberg N, Bakir I, Caselman F, et al. Higher levels of serum cytokines and myocardial tissue markers during on-pump versus off-pump coronary artery bypass surgery. J Card Surg. 2006;21:395-402.

E65. Niranjan G, Asimakopoulos G, Karagounis A, Cockerill G, Thompson M Chandrasekaran V. Effects of cell saver autologous blood transfusion on blood loss and homologous blood transfusion requirements in patients undergoing cardiac surgery on- versus off-cardiopulmonary bypass: a randomised trial. Eur J Cardiothorac Surg. 2006;30:271-7.

E66. Nathoe HM, van Dijk D, Jansen EW, Suyker WJ, Diephuis JC, van Boven WJ, et al. A comparison of on-pump and off-pump coronary bypass surgery in low-risk patients. N Engl J Med. 2003;348:394-402.

E67. Lemma MG, Coscioni E, Tritto FP, Centofanti P, Fondacone C, Salica A, et al. On-pump versus off-pump coronary artery bypass surgery in high-risk patients: operative results of a prospective randomized trial (on-off study). $J$ Thorac Cardiovasc Surg. 2012;143:625-31.

E68. Onorati F, Rubino AS, Nucera S, Foti D, Sica V, Santini F, et al. Off-pump coronary artery bypass surgery versus standard linear or pulsatile cardiopulmonary bypass: endothelial activation and inflammatory response. Eur J Cardiothorac Surg. 2010;37:897-904.

E69. Ozkara C, Guler N, Batyraliev T, Okut H, Agirbasli M. Does off-pump coronary artery bypass surgery reduce secretion of plasminogen activator inhibitor-1? Int J Clin Pract. 2007;61:763-7.

E70. Paparella D, Galeone A, Venneri MT, Coviello M, Scrascia G, Marraudino N et al. Activation of the coagulation system during coronary artery bypass grafting: comparison between on-pump and off-pump techniques. J Thorac Cardiovasc Surg. 2006;131:290-7.

E71. Parolari A, Alamanni F, Juliano G, Polvani G, Roberto M, Veglia F, et al Oxygen metabolism during and after cardiac surgery: role of CPB. Ann Thorac Surg. 2003;76:737-43.

E72. Parolari A, Mussoni L, Frigerio M, Naliato M, Alamanni F, Polvani GL, et al The role of tissue factor and P-selectin in the procoagulant response that occurs in the first month after on-pump and off-pump coronary artery bypass grafting. J Thorac Cardiovasc Surg. 2005;130:1561-6.

E73. Penttilä HJ, Lepojärvi MV, Kiviluoma KT, Kaukoranta PK, Hassinen IE, Peuhkurinen KJ. Myocardial preservation during coronary surgery with and without cardiopulmonary bypass. Ann Thorac Surg. 2001;71:565-71.

E74. Straka Z, Widimsky P, Jirasek K, Stros P, Votava J, Vanek T, et al. Off-pump versus on-pump coronary surgery: final results from a prospective randomized study PRAGUE-4. Ann Thorac Surg. 2004;77:789-93.

E75. Hlavicka J, Straka Z, Jelinek S, Budera P, Vanek T, Widimsky P. PPRAGUE-6 trial: off-pump versus on-pump coronary artery bypass graft surgery in patients with EuroSCORE $\geq 6$. Available at: http://my.americanheart.org/ idc/groups/ahamah-public/@wcm/@sop/@scon/documents/downloadable/ ucm_450009.pdf. Accessed March 12, 2013.

E76. Bednar F, Osmancik P, Vanek T, Mocikova H, Jares M, Straka Z, et al. Platele activity and aspirin efficacy after off-pump compared with on-pump coronary artery bypass surgery: results from the prospective randomized trial PRAGUE 11-Coronary Artery Bypass and REactivity of Thrombocytes (CABARET). $J$ Thorac Cardiovasc Surg. 2008;136:1054-60.

E77. Sousa Uva M, Cavaco S, Oliveira AG, Matias F, Silva C, Mesquita A, et al. Early graft patency after off-pump and on-pump coronary bypass surgery: a prospective randomized study. Eur Heart J. 2010;31:2492-9.

E78. Rachwalik M, Lysenko L, Kustrzycki W, Pelczar M, Wachnik J. Comparison of spirometry in patients after on-pump and off-pump coronary artery bypass grafting. Anesth Int Ther. 2006;38:4-7.

E79. Rainio A, Hautala N, Pelkonen O, Palosaari T, Heikkinen J, Mosorin M, et al Risk of retinal microembolism after off-pump and on-pump coronary artery bypass surgery. J Cardiovasc Surg (Torino). 2007;48:773-9. 
E80. Raja SG, Haider Z, Ahmad M. Predictors of gastrointestinal complications after conventional and beating heart coronary surgery. Surgeon. 2003;1: 221-8.

E81. Rasmussen BS, Laugesen H, Sollid J, Gronlund J, Rees SE, Toft E, et al. Oxygenation and release of inflammatory mediators after off-pump compared with after on-pump coronary artery bypass surgery. Acta Anaesthesiol Scand. 2007;51:1202-10.

E82. Rastan AJ, Bittner HB, Gummert JF, Walther T, Schewick CV, Girdauskas E, et al. On-pump beating heart versus off-pump coronary artery bypass surgery-evidence of pump-induced myocardial injury. Eur J Cardiothorac Surg. 2005;27:1057-64.

E83. Shroyer AL, Grover FL, Hattler B, Collins JF, McDonald GO, Kozora E, et al On-pump versus off-pump coronary-artery bypass surgery. $N$ Engl J Med. 2009;361:1827-37.

E84. Sahlman A, Ahonen J, Nemlander A, Salmenperä M, Eriksson H, Rämö J, et al. Myocardial metabolism on off-pump surgery; a randomized study of 50 cases. Scand Cardiovasc J. 2003;37:211-5.

E85. Sajja LR, Mannam G, Chakravarthi RM, Sompalli S, Naidu SK, Somaraju B, et al. Coronary artery bypass grafting with or without cardiopulmonary bypass in patients with preoperative non-dialysis dependent renal insufficiency: a randomized study. J Thorac Cardiovasc Surg. 2007;133:378-88.

E86. Selvanayagam JB, Petersen SE, Francis JM, Robson MD, Kardos A, Neubauer S, et al. Effects of off-pump versus on-pump coronary surgery on reversible and irreversible myocardial injury: a randomized trial using cardiovascular magnetic resonance imaging and biochemical markers. Circulation. 2004;109:345-50.

E87. Puskas JD, Williams WH, Duke PG, Staples JR, Glas KE, Marshall JJ, et al. Off-pump coronary artery bypass grafting provides complete revascularization with reduced myocardial injury, transfusion requirements, and length of stay: a prospective randomized comparison of two hundred unselected patients undergoing off-pump versus conventional coronary artery bypass grafting. J Thorac Cardiovasc Surg. 2003;125:797-808.

E88. Syed A, Fawzy H, Farag A, Nemlander A. Comparison of pulmonary gas exchange in OPCAB versus conventional CABG. Heart Lung Circ. 2004; 13:168-72.

E89. Tang AT, Knott J, Nanson J, Hsu J, Haw MP, Ohri SK. A prospective randomized study to evaluate the renoprotective action of beating heart coronary surgery in low risk patients. Eur J Cardiothorac Surg. 2002;22:118-23.

E90. Tatoulis J, Rice S, Davis P, Goldblatt JC, Marasco S. Patterns of postoperative systemic vascular resistance in a randomized trial of conventional on-pump versus off-pump coronary artery bypass graft surgery. Ann Thorac Surg. 2006;82:1436-44.

E91. Tully PJ, Baker RA, Kneebone AC, Knight JL. Neuropsychologic and quality-of-life outcomes after coronary artery bypass surgery with and without cardiopulmonary bypass: a prospective randomized trial. J Cardiothorac Vasc Anesth. 2008;22:515-21.
E92. van Boven WJ, Morariu A, Salzberg SP, Gerritsen WB, Waanders FG, Korse TC, et al. Impact of different surgical strategies on perioperative protein S100ß release in elderly patients undergoing coronary artery bypass grafting. Innovations (Phila). 2013;8:230-6.

E93. van Boven WJ, Gerritsen WB, Driessen AH, van Dongen EP, Klautz RJ, Aarts LP. Minimised closed circuit coronary artery bypass grafting in the elderly is associated with lower levels of organ-specific biomarkers: a prospective randomised study. Eur J Anaesthesiol. 2013;30:685-94.

E94. Vedin J, Nyman H, Ericsson A, Hylander S, Vaage J. Cognitive function after on or off pump coronary artery bypass grafting. Eur J Cardiothorac Surg. 2006;30:305-10

E95. Velissaris T, Tang A, Murray M, El-Minshawy A, Hett D, Ohri S. A prospective randomized study to evaluate splanchnic hypoxia during beating-heart and conventional coronary revascularization. Eur J Cardiothorac Surg. 2003;23:917-24.

E96. Velissaris T, Tang AT, Murray M, Mehta RL, Wood PJ, Hett DA, et al. A prospective randomized study to evaluate stress response during beatingheart and conventional coronary revascularization. Ann Thorac Surg. 2004; 78:506-12.

E97. Vural KM, Tasdemir O, Karagöz H, Emir M, Tarcan O, Bayazit K. Comparison of the early results of coronary artery bypass grafting with and without extracorporeal circulation. Thorac Cardiovasc Surg. 1995;43:320-5.

E98. Wan IY, Arifi AA, Wan S, Yip JH, Sihoe AD, Thung KH, et al. Beating heart revascularization with or without cardiopulmonary bypass: evaluation of inflammatory response in a prospective randomized study. J Thorac Cardiovasc Surg. 2004;127:1624-31.

E99. Wandschneider W, Thalmann M, Trampitsch E, Ziervogel G, Kobinia G. Off-pump coronary bypass operations significantly reduce S100 release: an indicator for less cerebral damage? Ann Thorac Surg. 2000;70:1577-9.

E100. Wehlin L, Vedin J, Vaage J, Lundahl J. Activation of complement and leukocyte receptors during on- and off pump coronary artery bypass surgery. Eur J Cardiothorac Surg. 2004;25:35-42.

E101. Wehlin L, Vedin J, Vaage J, Lundahl J. Peripheral blood monocyte activation during coronary artery bypass grafting with or without cardiopulmonary bypass. Scand Cardiovasc J. 2005;39:78-86.

E102. Zamvar V, Williams D, Hall J, Payne N, Cann C, Young K, et al. Assessment of neurocognitive impairment after off-pump and on-pump techniques for coronary artery bypass graft surgery: prospective randomised controlled trial. BMJ. 2002;325:1268.

E103. Yu L, Gu T, Shi E, Wang C, Fang Q, Yu Y, et al. Off-pump versus on-pump coronary artery bypass surgery in patients with triple-vessel disease and enlarged ventricles. Ann Saudi Med. 2014;34:222-8.

E104. Moller CH, Penninga L, Wetterslev J, Steinbrüchel DA, Gluud C. Clinical outcomes in randomized trials of off- vs. on-pump coronary artery bypass surgery: systematic review with meta-analyses and trial sequential analyses. Eur Heart J. 2008;29:2601-16. 


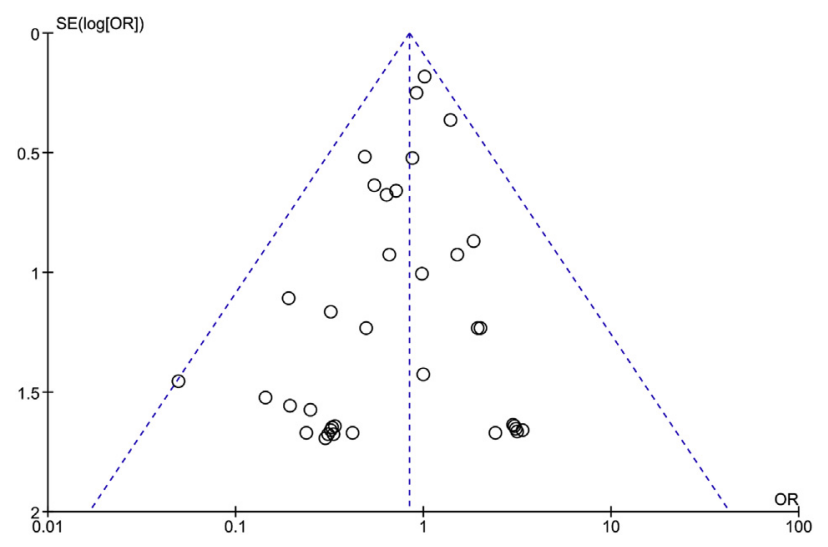

APPENDIX FIGURE 1. Funnel plot for all-cause mortality. SE, Standard error; $O R$, odds ratio.

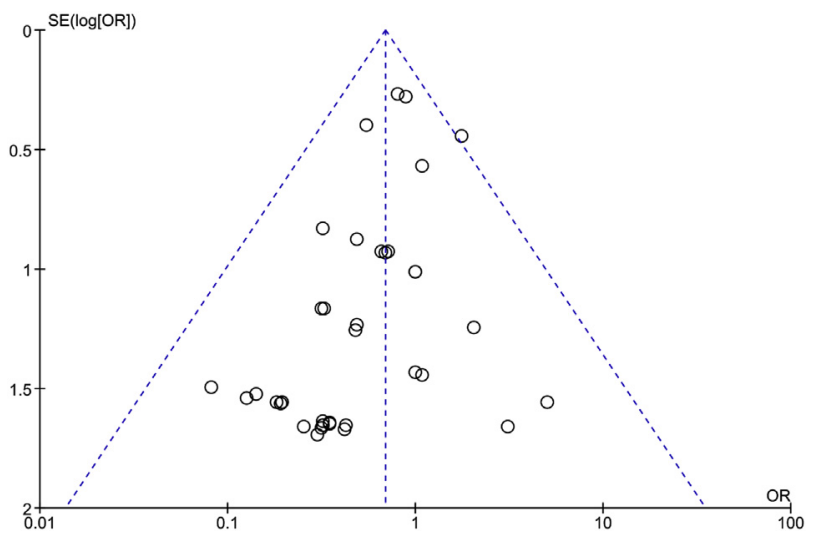

APPENDIX FIGURE 3. Funnel plot for cerebral stroke. SE, Standard error; $O R$, odds ratio.

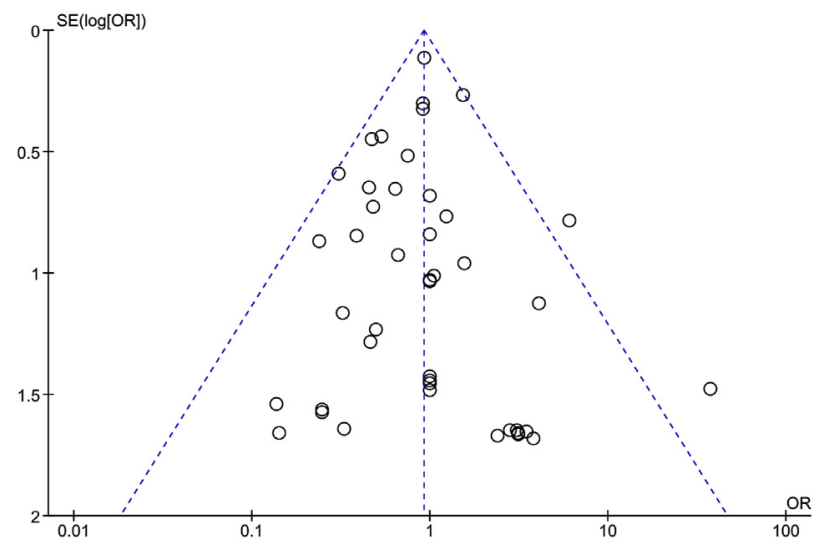

APPENDIX FIGURE 2. Funnel plot for MI. SE, Standard error; $O R$, odds ratio. 


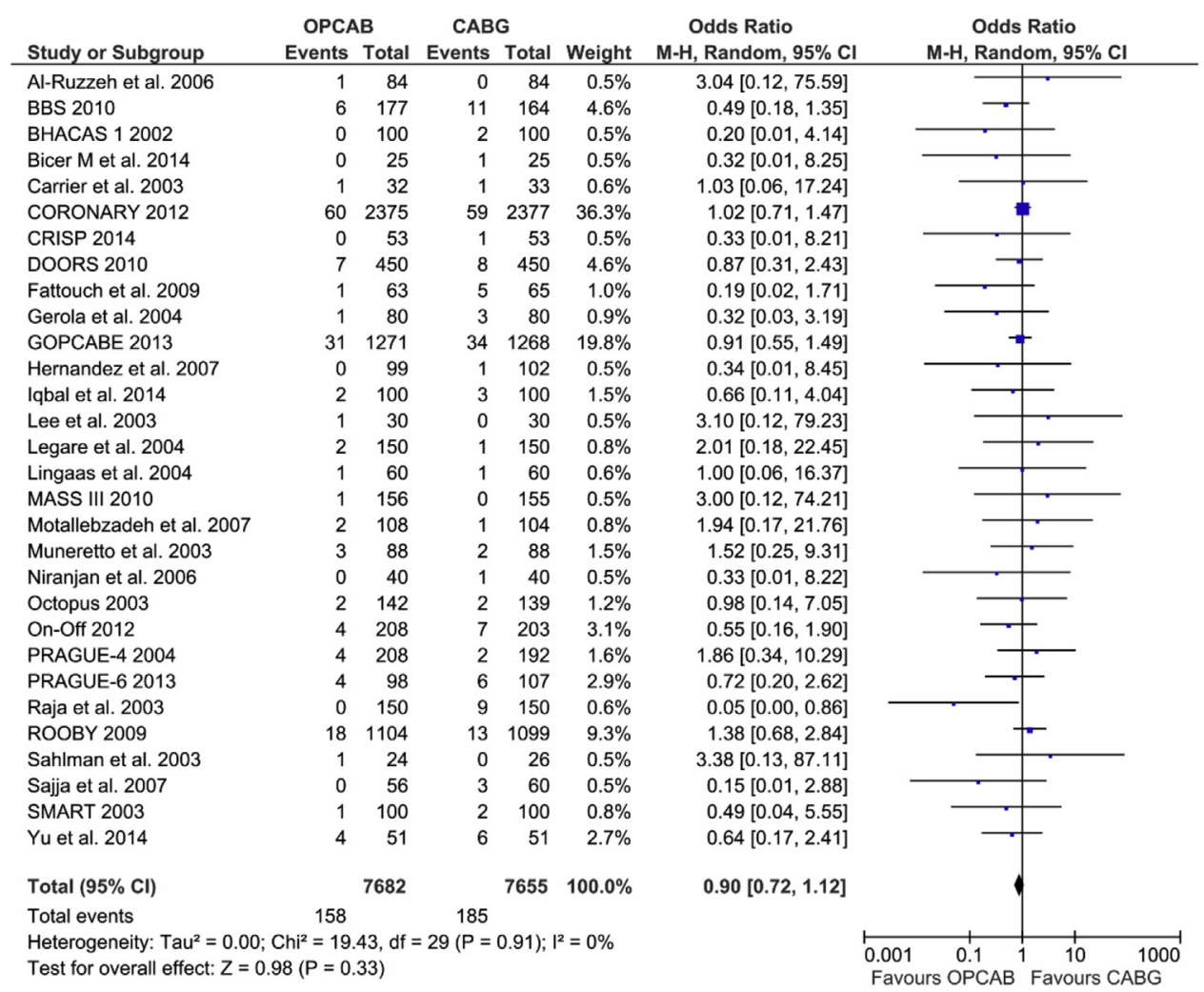

APPENDIX FIGURE 4. Sensitivity analysis of all-cause mortality after exclusion of studies with $<50$ patients. Size of squares is proportional to the statistical weight of each trial. Diamonds represent OR point estimates; horizontal lines represent $95 \%$ CIs for given point estimates. The positioning of both diamonds and squares (along with $95 \% \mathrm{CIs}$ ) beyond the vertical line (unit value) suggests significant outcome. $O P C A B$, Off-pump coronary artery bypass; $C A B G$, coronary artery bypass grafting; $M-H$, Mantel-Haenszel; $C I$, confidence interval; $B B S$, best bypass surgery trial; $B H A C A S 1$, beating heart against cardioplegic arrest study 1; CORONARY, CABG off or on pump revascularization study; CRISP, The Coronary artery bypass grafting in high-RISk patients randomized to off- or on-Pump surgery; DOORS, Danish on-pump versus off-pump randomization study; GOPCABE; German off-pump coronary artery bypass grafting in elderly patients; MASS III, off-pump and on-pump stable multivessel coronary artery bypass grafting; Octopus, comparison of on-pump and off-pump CORONARY bypass surgery in low-risk patients; PRAGUE-4, A randomized comparison between off-pump and on-pump surgery PRAGUE-4; PRAGUE-6, Off-pump versus on-pump coronary artery bypass graft surgery in patients with EuroSCORE $\geq 6$; ROOBY, veterans affairs randomized on/off bypass trial; SMART, surgical management of arterial revascularization therapies; $d f$, degrees of freedom. 


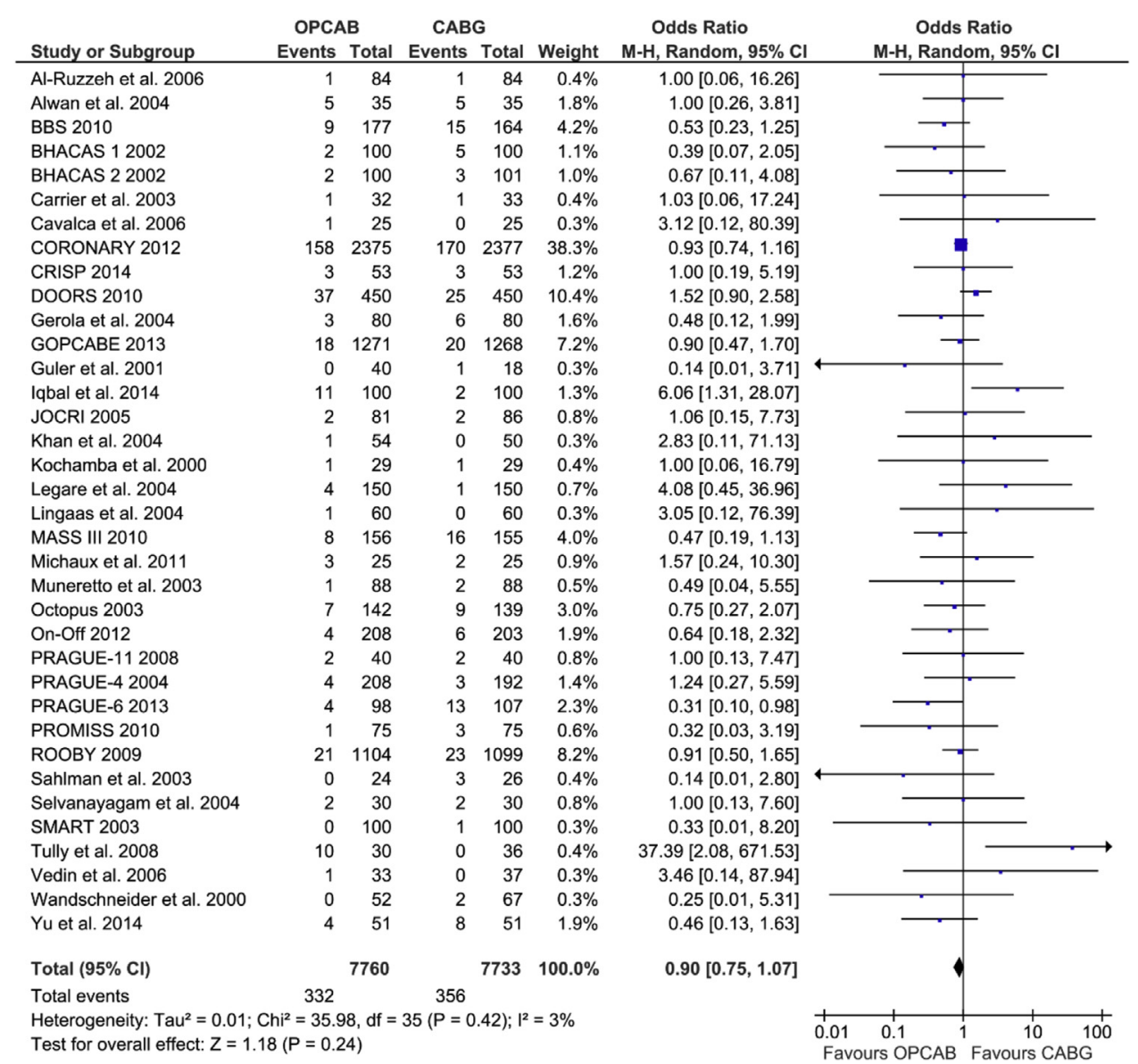

APPENDIX FIGURE 5. Sensitivity analysis of MI after exclusion of studies with $<50$ patients. Size of squares is proportional to the statistical weight of each trial. Diamonds represent OR point estimates; horizontal lines represent $95 \%$ CIs for given point estimates. The positioning of both diamonds and squares (along with $95 \% \mathrm{CIs}$ ) beyond the vertical line (unit value) suggests significant outcome. OPCAB, Off-pump coronary artery bypass; $C A B G$, coronary artery bypass grafting; $M-H$, Mantel-Haenszel; $C I$, confidence interval; $B B S$, best bypass surgery trial; BHACAS 1 , beating heart against cardioplegic arrest study 1; BHACAS 2, eating heart against cardioplegic arrest study 2; CORONARY, CABG off or on pump revascularization study; CRISP, The Coronary artery bypass grafting in high-RISk patients randomized to off- or on-Pump surgery; DOORS, Danish on-pump versus off-pump randomization study; GOPCABE; German off-pump coronary artery bypass grafting in elderly patients; JOCRI, Japanese off-pump coronary revascularization investigation study; MASS III, off-pump and on-pump stable multivessel coronary artery bypass grafting; Octopus, comparison of on-pump and off-pump CORONARY bypass surgery in low-risk patients; PRAGUE-11, A randomized comparison between off-pump and on-pump surgery PRAGUE-11; PRAGUE-4, A randomized comparison between off-pump and on-pump surgery PRAGUE-4; PRAGUE-6, Off-pump versus on-pump coronary artery bypass graft surgery in patients with EuroSCORE $\geq 6$; PROMISS, The prospective randomized comparison of off-pump and on-pump multivessel coronary artery bypass surgery; $R O O B Y$, veterans affairs randomized on/off bypass trial; SMART, surgical management of arterial revascularization therapies; $d f$, degrees of freedom. 


\begin{tabular}{|c|c|c|c|c|c|c|c|c|}
\hline Study or Subgroup & \multicolumn{2}{|c|}{ OPCAB } & \multicolumn{2}{|c|}{ CABG } & Weight & $\begin{array}{c}\text { Odds Ratio } \\
\text { M-H, Random, } 95 \% \mathrm{Cl}\end{array}$ & \multicolumn{2}{|c|}{$\begin{array}{c}\text { Odds Ratio } \\
\mathrm{M}-\mathrm{H}, \text { Random, } 95 \% \mathrm{CI}\end{array}$} \\
\hline Al-Ruzzeh et al. 2006 & 2 & 84 & 2 & 84 & $1.6 \%$ & $1.00[0.14,7.27]$ & & \\
\hline BBS 2010 & 7 & 177 & 6 & 164 & $5.1 \%$ & $1.08[0.36,3.30]$ & & \\
\hline BHACAS 12002 & 2 & 100 & 3 & 100 & $1.9 \%$ & $0.66[0.11,4.04]$ & & \\
\hline BHACAS 22002 & 1 & 100 & 3 & 101 & $1.2 \%$ & $0.33[0.03,3.23]$ & & \\
\hline Bicer M et al. 2014 & 0 & 25 & 3 & 25 & $0.7 \%$ & $0.13[0.01,2.58]$ & $\longleftarrow$ & \\
\hline Carrier et al. 2003 & 0 & 32 & 1 & 33 & $0.6 \%$ & $0.33[0.01,8.49]$ & & \\
\hline CORONARY 2012 & 24 & 2375 & 27 & 2377 & $20.7 \%$ & $0.89[0.51,1.54]$ & & \\
\hline CRISP 2014 & 2 & 53 & 1 & 53 & $1.1 \%$ & $2.04[0.18,23.19]$ & & \\
\hline DOORS 2010 & 10 & 450 & 18 & 450 & $10.3 \%$ & $0.55[0.25,1.20]$ & & \\
\hline GOPCABE 2013 & 26 & 1271 & 32 & 1268 & $23.1 \%$ & $0.81[0.48,1.36]$ & & \\
\hline Hernandez et al. 2007 & 0 & 99 & 3 & 102 & $0.7 \%$ & $0.14[0.01,2.80]$ & & \\
\hline Iqbal et al. 2014 & 2 & 100 & 6 & 100 & $2.4 \%$ & $0.32[0.06,1.62]$ & & - \\
\hline JOCRI 2005 & 0 & 81 & 1 & 86 & $0.6 \%$ & $0.35[0.01,8.71]$ & & \\
\hline Jongman et al. 2014 & 0 & 30 & 1 & 30 & $0.6 \%$ & $0.32[0.01,8.24]$ & & \\
\hline Kok et al. 2014 & 0 & 30 & 1 & 30 & $0.6 \%$ & $0.32[0.01,8.24]$ & & \\
\hline Lee et al. 2003 & 0 & 30 & 1 & 30 & $0.6 \%$ & $0.32[0.01,8.24]$ & & \\
\hline Legare et al. 2004 & 2 & 150 & 0 & 150 & $0.7 \%$ & $5.07[0.24,106.45]$ & & \\
\hline Lingaas et al. 2004 & 0 & 60 & 2 & 60 & $0.7 \%$ & $0.19[0.01,4.11]$ & & \\
\hline Lund et al. 2003 & 0 & 29 & 1 & 23 & $0.6 \%$ & $0.25[0.01,6.54]$ & & \\
\hline MASS III 2010 & 2 & 156 & 4 & 155 & $2.2 \%$ & $0.49[0.09,2.72]$ & & \\
\hline Medved et al. 2008 & 1 & 30 & 2 & 30 & $1.1 \%$ & $0.48[0.04,5.63]$ & & \\
\hline Michaux et al. 2011 & 1 & 25 & 0 & 25 & $0.6 \%$ & $3.12[0.12,80.39]$ & & \\
\hline Motallebzadeh et al. 2007 & 1 & 108 & 3 & 104 & $1.2 \%$ & $0.31[0.03,3.07]$ & & \\
\hline Muneretto et al. 2003 & 0 & 88 & 2 & 88 & $0.7 \%$ & $0.20[0.01,4.13]$ & & \\
\hline Nesher et al. 2006 & 2 & 61 & 3 & 64 & $1.9 \%$ & $0.69[0.11,4.27]$ & & \\
\hline Niranjan et al. 2006 & 1 & 40 & 1 & 40 & $0.8 \%$ & $1.00[0.06,16.56]$ & & \\
\hline Octopus 2003 & 1 & 142 & 2 & 139 & $1.1 \%$ & $0.49[0.04,5.42]$ & & \\
\hline On-Off 2012 & 0 & 208 & 1 & 203 & $0.6 \%$ & $0.32[0.01,7.99]$ & & \\
\hline PRAGUE-4 2004 & 0 & 208 & 2 & 192 & $0.7 \%$ & $0.18[0.01,3.83]$ & & \\
\hline PRAGUE-6 2013 & 2 & 98 & 3 & 107 & $1.9 \%$ & $0.72[0.12,4.42]$ & & \\
\hline PROMISS 2010 & 0 & 75 & 2 & 75 & $0.7 \%$ & $0.19[0.01,4.12]$ & & \\
\hline ROOBY 2009 & 14 & 1104 & 8 & 1099 & $8.3 \%$ & $1.75[0.73,4.19]$ & & \\
\hline Sahlman et al. 2003 & 1 & 24 & 1 & 26 & $0.8 \%$ & $1.09[0.06,18.40]$ & & \\
\hline Sajja et al. 2007 & 0 & 56 & 1 & 60 & $0.6 \%$ & $0.35[0.01,8.80]$ & & \\
\hline Selvanayagam et al. 2004 & 0 & 30 & 1 & 30 & $0.6 \%$ & $0.32[0.01,8.24]$ & & \\
\hline SMART 2003 & 2 & 100 & 2 & 100 & $1.6 \%$ & $1.00[0.14,7.24]$ & & \\
\hline Yu et al. 2014 & 0 & 51 & 5 & 51 & $0.7 \%$ & $0.08[0.00,1.53]$ & $\longleftarrow$ & \\
\hline Total $(95 \% \mathrm{Cl})$ & & 7880 & & 7854 & $100.0 \%$ & $0.73[0.56,0.93]$ & 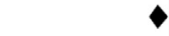 & \\
\hline Total events & 106 & & 155 & & & & & \\
\hline $\begin{array}{l}\text { Heterogeneity: } \operatorname{Tau}^{2}=0.00 \\
\text { Test for overall effect: } Z=2\end{array}$ & $\begin{array}{l}\mathrm{Chi}^{2}=21 \\
50(\mathrm{P}=0 . \mathrm{C}\end{array}$ & $\begin{array}{l}\text { 19, df }= \\
01)\end{array}$ & $=36(P=C$ & $0.98) ; 1^{2}$ & $=0 \%$ & & $\begin{array}{ll}0.01 & 0.1 \\
\text { Favours OPCAB }\end{array}$ & $1 \frac{10}{\text { Favours C }}$ \\
\hline
\end{tabular}

APPENDIX FIGURE 6. Sensitivity analysis of cerebral stroke after exclusion of studies with $<50$ patients. Size of squares is proportional to the statistical weight of each trial. Diamonds represent OR point estimates; horizontal lines represent $95 \%$ CIs for given point estimates. The positioning of both diamonds and squares (along with $95 \% \mathrm{CIs}$ ) beyond the vertical line (unit value) suggests significant outcome. OPCAB, Off-pump coronary artery bypass; $C A B G$, coronary artery bypass grafting; $M-H$, Mantel-Haenszel; $C I$, confidence interval; $B B S$, best bypass surgery trial; BHACAS 1 , beating heart against cardioplegic arrest study 1; BHACAS 2, eating heart against cardioplegic arrest study 2; CORONARY, CABG off or on pump revascularization study; CRISP, The Coronary artery bypass grafting in high-RISk patients randomized to off- or on-Pump surgery; DOORS, Danish on-pump versus off-pump randomization study; GOPCABE; German off-pump coronary artery bypass grafting in elderly patients; JOCRI, Japanese off-pump coronary revascularization investigation study; MASS III, off-pump and on-pump stable multivessel coronary artery bypass grafting; Octopus, comparison of on-pump and off-pump CORONARY bypass surgery in low-risk patients; PRAGUE-4, A randomized comparison between off-pump and on-pump surgery PRAGUE-4; PRAGUE-6, Off-pump versus on-pump coronary artery bypass graft surgery in patients with EuroSCORE $\geq 6$; PROMISS, The prospective randomized comparison of off-pump and on-pump multivessel coronary artery bypass surgery; ROOBY, veterans affairs randomized on/off bypass trial; SMART, surgical management of arterial revascularization therapies; $d f$, degrees of freedom. 


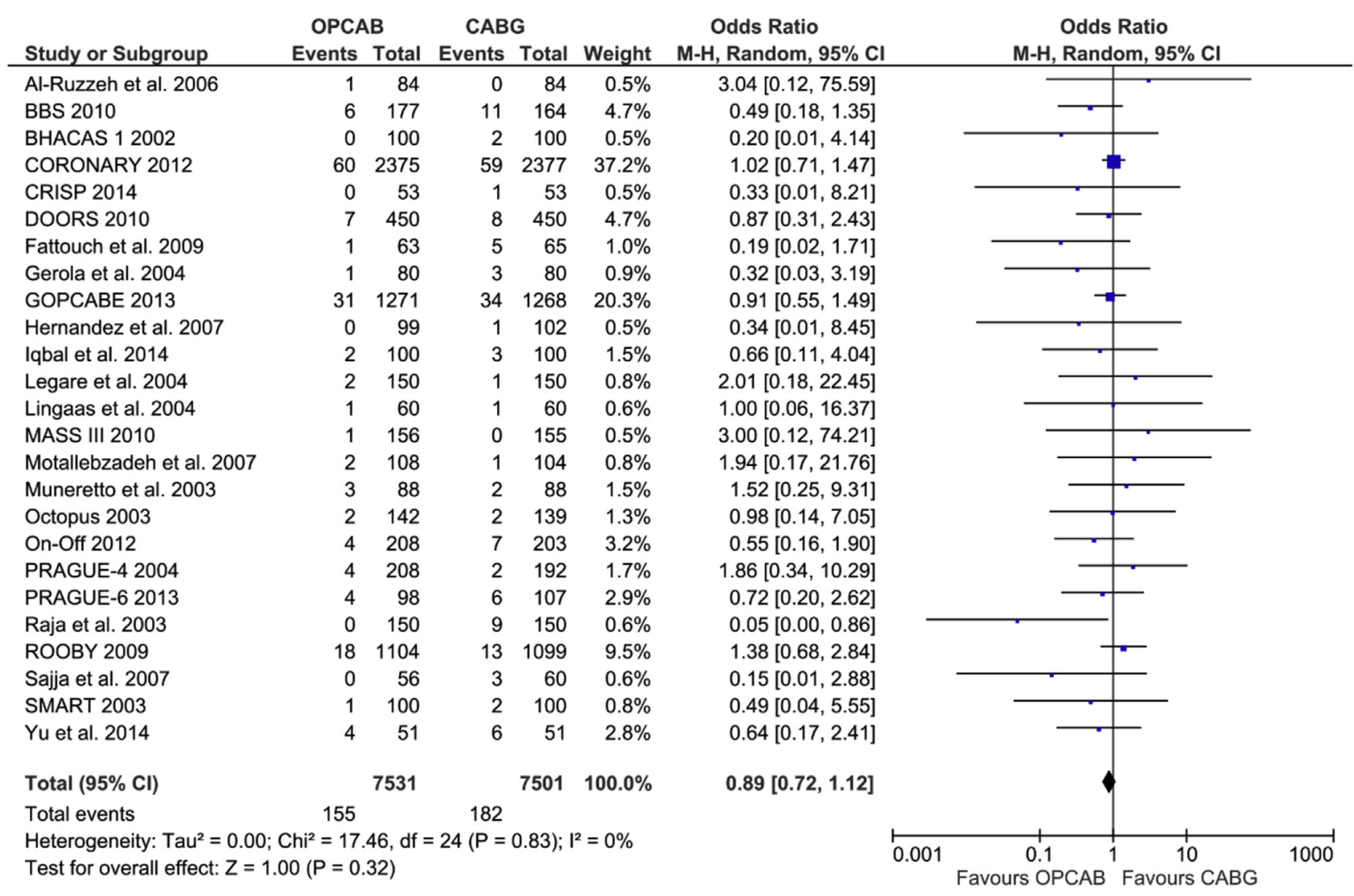

APPENDIX FIGURE 7. Sensitivity analysis of all-cause mortality after exclusion of studies with $<100$ patients. Size of squares is proportional to the statistical weight of each trial. Diamonds represent OR estimates; horizontal lines represent $95 \%$ CIs for given point estimates. The positioning of both diamonds and squares (along with $95 \% \mathrm{CIs}$ ) beyond the vertical line (unit value) suggests significant outcome. OPCAB, Off-pump coronary artery bypass; $C A B G$, coronary artery bypass grafting; $M-H$, Mantel-Haenszel; $C I$, confidence interval; $B B S$, best bypass surgery trial; BHACAS 1 , beating heart against cardioplegic arrest study 1; CORONARY, CABG off or on pump revascularization study; CRISP, The Coronary artery bypass grafting in high-RISk patients randomised to off- or on-Pump surgery; DOORS, Danish on-pump versus off-pump randomization study; GOPCABE; German off-pump coronary artery bypass grafting in elderly patients; MASS III, off-pump and on-pump stable multivessel coronary artery bypass grafting; Octopus, comparison of onpump and off-pump CORONARY bypass surgery in low-risk patients; PRAGUE-4, A randomized comparison between off-pump and on-pump surgery PRAGUE-4; PRAGUE-6, Off-pump versus on-pump coronary artery bypass graft surgery in patients with EuroSCORE $\geq 6$; ROOBY, veterans affairs randomized on/off bypass trial; SMART, surgical management of arterial revascularization therapies; $d f$, degrees of freedom. 
OPCAB

CABG

Study or Subgroup

Events Total Events Total Weight M-H, Random, 95\% Cl

Al-Ruzzeh et al. 2006

$\begin{array}{lllll}1 & 84 & 1 & 84 & 0.5 \%\end{array}$

BBS 2010

$9 \quad 177$

15164

$4.7 \%$

BHACAS 12002

BHACAS 22002

CORONARY 2012

CRISP 2014

DOORS 2010

Gerola et al. 2004

GOPCABE 2013

Iqbal et al. 2014

JOCRI 2005

Khan et al. 2004

Legare et al. 2004

Lingaas et al. 2004

MASS III 2010

Muneretto et al. 2003

Octopus 2003

On-Off 2012

PRAGUE-4 2004

PRAGUE-6 2013

PROMISS 2010

ROOBY 2009

SMART 2003

Wandschneider et al. 2000

Yu et al. 2014

2100

$5100 \quad 13 \%$

2100

1582375

353

$37 \quad 450$

380

$18 \quad 1271$

11100

$\begin{array}{lll}5 & 100 & 1.3 \% \\ 3 & 101 & 1.1 \%\end{array}$

$170 \quad 2377 \quad 39.0 \%$

$3 \quad 53 \quad 1.3 \%$

$25 \quad 450 \quad 11.3 \%$

$6 \quad 80 \quad 1.7 \%$

$20 \quad 1268 \quad 8.0 \%$

$2100 \quad 1.5 \%$

$\begin{array}{lllll}2 & 81 & 2 & 86 & 0.9 \%\end{array}$

$\begin{array}{lllll}1 & 54 & 0 & 50 & 0.3 \%\end{array}$

$\begin{array}{lllll}4 & 150 & 1 & 150 & 0.7 \%\end{array}$

$\begin{array}{lllll}1 & 60 & 0 & 60 & 0.3 \%\end{array}$

$\begin{array}{lllll}8 & 156 & 16 & 155 & 4.4 \%\end{array}$

$\begin{array}{lllll}1 & 88 & 2 & 88 & 0.6 \%\end{array}$

$\begin{array}{lllll}7 & 142 & 9 & 139 & 3.4 \%\end{array}$

$\begin{array}{lllll}4 & 208 & 6 & 203 & 2.1 \%\end{array}$

$\begin{array}{lllll}4 & 208 & 3 & 192 & 1.6 \%\end{array}$

$\begin{array}{lllll}4 & 98 & 13 & 107 & 2.6 \%\end{array}$

$\begin{array}{lllll}1 & 75 & 3 & 75 & 0.7 \%\end{array}$

$\begin{array}{lllll}21 & 1104 & 23 & 1099 & 9.0 \%\end{array}$

$\begin{array}{lllll}0 & 100 & 1 & 100 & 0.3 \%\end{array}$

$\begin{array}{lllll}0 & 52 & 2 & 67 & 0.4 \%\end{array}$

$\begin{array}{lllll}4 & 51 & 8 & 51 & 2.2 \%\end{array}$

Total $(95 \% \mathrm{CI})$

Total events

7417

Heterogeneity: $\mathrm{Tau}^{2}=0.01 ; \mathrm{Ch}^{2}=25.13, \mathrm{df}=24(P=0.40) ; \mathrm{I}^{2}=5 \%$

Test for overall effect: $Z=1.36(P=0.17)$
Odds Ratio

Odds Ratio

M-H, Random, $95 \% \mathrm{Cl}$

$1.00[0.06,16.26]$

$0.53[0.23,1.25]$

$0.39[0.07,2.05]$

$0.67[0.11,4.08]$

$0.93[0.74,1.16]$

$1.00[0.19,5.19]$

$1.52[0.90,2.58]$

$0.48[0.12,1.99]$

$0.90[0.47,1.70]$

$6.06[1.31,28.07]$

$1.06[0.15,7.73]$

$2.83[0.11,71.13]$

$4.08[0.45,36.96]$

$3.05[0.12,76.39]$

$0.47[0.19,1.13]$

$0.49[0.04,5.55]$

$0.75[0.27,2.07]$

$0.64[0.18,2.32]$

$1.24[0.27,5.59]$

$0.31[0.10,0.98]$

$0.32[0.03,3.19]$

$0.91[0.50,1.65]$

$0.33[0.01,8.20]$

$0.25[0.01,5.31]$

$0.46[0.13,1.63]$

$0.88[0.72,1.06]$

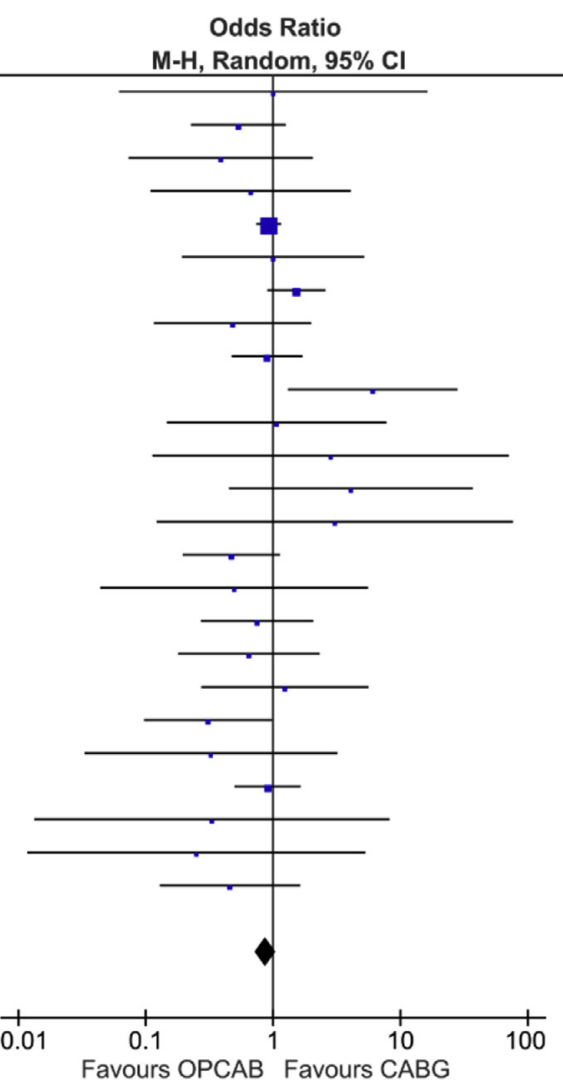

APPENDIX FIGURE 8. Sensitivity analysis of MI after exclusion of studies with $<100$ patients. Size of squares is proportional to the statistical weight of each trial. Diamonds represent OR point estimates; horizontal lines represent $95 \%$ CIs for given point estimates. The positioning of both diamonds and squares (along with $95 \% \mathrm{CIs}$ ) beyond the vertical line (unit value) suggests significant outcome. $O P C A B$, Off-pump coronary artery bypass; $C A B G$, coronary artery bypass grafting; $M-H$, Mantel-Haenszel; $C I$, confidence interval; $B B S$, best bypass surgery trial; BHACAS 1 , beating heart against cardioplegic arrest study 1; BHACAS 2, eating heart against cardioplegic arrest study 2; CORONARY, CABG off or on pump revascularization study; CRISP, The Coronary artery bypass grafting in high-RISk patients randomized to off- or on-Pump surgery; DOORS, Danish on-pump versus off-pump randomization study; GOPCABE; German off-pump coronary artery bypass grafting in elderly patients; JOCRI, Japanese off-pump coronary revascularization investigation study; MASS III, off-pump and on-pump stable multivessel coronary artery bypass grafting; Octopus, comparison of on-pump and off-pump CORONARY bypass surgery in low-risk patients; PRAGUE-4, A randomized comparison between off-pump and on-pump surgery PRAGUE-4; PRAGUE-6, Off-pump versus on-pump coronary artery bypass graft surgery in patients with EuroSCORE $\geq 6$; PROMISS, The prospective randomized comparison of off-pump and on-pump multivessel coronary artery bypass surgery; ROOBY, veterans affairs randomized on/off bypass trial; SMART, surgical management of arterial revascularization therapies; $d f$, degrees of freedom. 


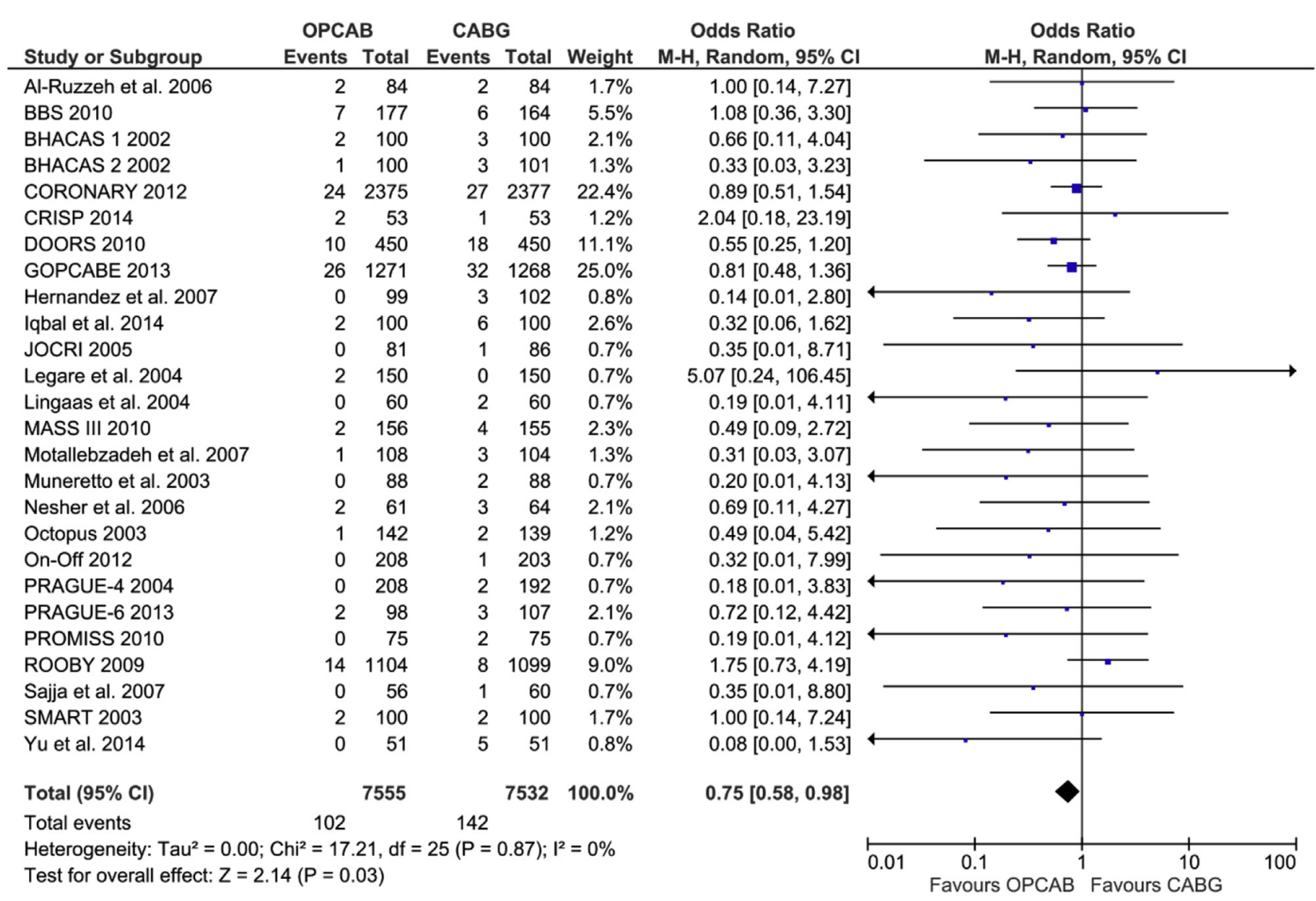

APPENDIX FIGURE 9. Sensitivity analysis of cerebral stroke after exclusion of studies with $<100$ patients. Size of squares is proportional to the statistical weight of each trial. Diamonds represent OR point estimates; horizontal lines represent $95 \%$ CIs for given point estimate. The positioning of both diamonds and squares (along with $95 \%$ CIs) beyond the vertical line (unit value) suggests significant outcome. OPCAB, Off-pump coronary artery bypass; $C A B G$, coronary artery bypass grafting; $M-H$, Mantel-Haenszel; $C I$, confidence interval; $B B S$, best bypass surgery trial; $B H A C A S 1$, beating heart against cardioplegic arrest study 1; BHACAS 2, eating heart against cardioplegic arrest study 2; CORONARY, CABG off or on pump revascularization study; CRISP, The Coronary artery bypass grafting in high-RISk patients randomized to off- or on-Pump surgery; DOORS, Danish on-pump versus off-pump randomization study; GOPCABE; German off-pump coronary artery bypass grafting in elderly patients; JOCRI, Japanese off-pump coronary revascularization investigation study; MASS III, off-pump and on-pump stable multivessel coronary artery bypass grafting; Octopus, comparison of on-pump and offpump CORONARY bypass surgery in low-risk patients; PRAGUE-4, A randomized comparison between off-pump and on-pump surgery PRAGUE-4; PRAGUE-6, Off-pump versus on-pump coronary artery bypass graft surgery in patients with EuroSCORE $\geq 6$; PROMISS, The prospective randomized comparison of off-pump and on-pump multivessel coronary artery bypass surgery; $R O O B Y$, veterans affairs randomized on/off bypass trial; SMART, surgical management of arterial revascularization therapies; $d f$, degrees of freedom. 
Study name

\begin{tabular}{|c|c|c|c|c|c|c|c|}
\hline & $\begin{array}{l}\text { Std diff } \\
\text { in means }\end{array}$ & $\begin{array}{l}\text { Standard } \\
\text { error }\end{array}$ & Variance & $\begin{array}{l}\text { Lower } \\
\text { limit }\end{array}$ & $\begin{array}{c}\text { Upper } \\
\text { limit }\end{array}$ & z-Value & p-Value \\
\hline Al-Ruzzeh et al. 2006 & 0.612 & 0.904 & 0.818 & -1.160 & 2.385 & 0.677 & 0.498 \\
\hline BBS 2010 & -0.396 & 0.286 & 0.082 & -0.957 & 0.166 & -1.381 & 0.167 \\
\hline BHACAS 12002 & -0.898 & 0.858 & 0.736 & -2.579 & 0.783 & -1.047 & 0.295 \\
\hline Bicer M et al. 2014 & -0.628 & 0.914 & 0.835 & -2.419 & 1.163 & -0.687 & 0.492 \\
\hline Carrier et al. 2003 & -0.766 & 0.865 & 0.749 & -2.462 & 0.930 & -0.886 & 0.376 \\
\hline CORONARY 2012 & 0.010 & 0.102 & 0.010 & -0.191 & 0.211 & 0.097 & 0.922 \\
\hline Covino et al. 2001 & -0.786 & 0.919 & 0.844 & -2.587 & 1.015 & -0.856 & 0.392 \\
\hline CRISP 2014 & -0.616 & 0.907 & 0.822 & -2.393 & 1.161 & -0.680 & 0.497 \\
\hline Czerny et al. 2000 & -0.642 & 0.923 & 0.851 & -2.451 & 1.166 & -0.696 & 0.486 \\
\hline DOORS 2010 & -0.075 & 0.288 & 0.083 & -0.639 & 0.489 & -0.260 & 0.795 \\
\hline Fattouch et al. 2009 & -0.905 & 0.612 & 0.375 & -2.105 & 0.294 & -1.479 & 0.139 \\
\hline Gerola et al. 2004 & -0.620 & 0.643 & 0.413 & -1.880 & 0.640 & -0.964 & 0.335 \\
\hline GOPCABE 2013 & -0.054 & 0.139 & 0.019 & -0.325 & 0.218 & -0.386 & 0.699 \\
\hline Hernandez et al. 2007 & -0.595 & 0.904 & 0.817 & -2.366 & 1.176 & -0.658 & 0.510 \\
\hline Iqbal et al. 2014 & -0.229 & 0.509 & 0.260 & -1.228 & 0.769 & -0.450 & 0.653 \\
\hline Jares et al. 2006 & -0.661 & 0.934 & 0.872 & -2.491 & 1.169 & -0.708 & 0.479 \\
\hline Lee et al. 2003 & 0.624 & 0.912 & 0.831 & -1.162 & 2.411 & 0.685 & 0.494 \\
\hline Legare et al. 2004 & 0.386 & 0.678 & 0.460 & -0.944 & 1.715 & 0.569 & 0.569 \\
\hline Lingaas et al. 2004 & 0.000 & 0.786 & 0.618 & -1.541 & 1.541 & 0.000 & 1.000 \\
\hline MASS III 2010 & 0.606 & 0.902 & 0.814 & -1.163 & 2.375 & 0.671 & 0.502 \\
\hline Motallebzadeh et al. 2004 & -0.479 & 0.920 & 0.846 & -2.282 & 1.323 & -0.521 & 0.602 \\
\hline Motallebzadeh et al. 2007 & 0.366 & 0.680 & 0.462 & -0.966 & 1.698 & 0.539 & 0.590 \\
\hline Muneretto et al. 2003 & 0.230 & 0.510 & 0.260 & -0.770 & 1.230 & 0.451 & 0.652 \\
\hline Niranjan et al. 2006 & -0.619 & 0.909 & 0.826 & -2.401 & 1.162 & -0.682 & 0.495 \\
\hline Octopus 2003 & -0.012 & 0.555 & 0.308 & -1.100 & 1.076 & -0.022 & 0.983 \\
\hline On-Off 2012 & -0.331 & 0.350 & 0.122 & -1.016 & 0.355 & -0.945 & 0.345 \\
\hline Paparella et al. 2006 & -0.606 & 0.922 & 0.850 & -2.412 & 1.201 & -0.657 & 0.511 \\
\hline PRAGUE-4 2004 & 0.343 & 0.481 & 0.231 & -0.599 & 1.285 & 0.713 & 0.476 \\
\hline PRAGUE-6 2013 & -0.184 & 0.365 & 0.133 & -0.898 & 0.531 & -0.505 & 0.614 \\
\hline Raja et al. 2003 & -1.657 & 0.803 & 0.644 & -3.230 & -0.084 & -2.065 & 0.039 \\
\hline Rastan et al. 2005 & 0.633 & 0.917 & 0.841 & -1.164 & 2.431 & 0.691 & 0.490 \\
\hline ROOBY 2009 & 0.179 & 0.202 & 0.041 & -0.217 & 0.575 & 0.888 & 0.375 \\
\hline Sahlman et al. 2003 & 0.672 & 0.914 & 0.835 & -1.119 & 2.463 & 0.735 & 0.462 \\
\hline Sajja et al. 2007 & -1.063 & 0.840 & 0.705 & -2.709 & 0.583 & -1.266 & 0.206 \\
\hline SMART 2003 & -0.388 & 0.680 & 0.462 & -1.720 & 0.945 & -0.570 & 0.568 \\
\hline Wehlin et al. 2004 & 0.486 & 0.919 & 0.844 & -1.314 & 2.286 & 0.529 & 0.597 \\
\hline \multirow[t]{2}{*}{ Yu et al. 2014} & -0.248 & 0.374 & 0.140 & -0.981 & 0.486 & -0.662 & 0.508 \\
\hline & -0.071 & 0.061 & 0.004 & -0.190 & 0.048 & -1.175 & 0.240 \\
\hline
\end{tabular}

Std diff in means and $95 \% \mathrm{Cl}$
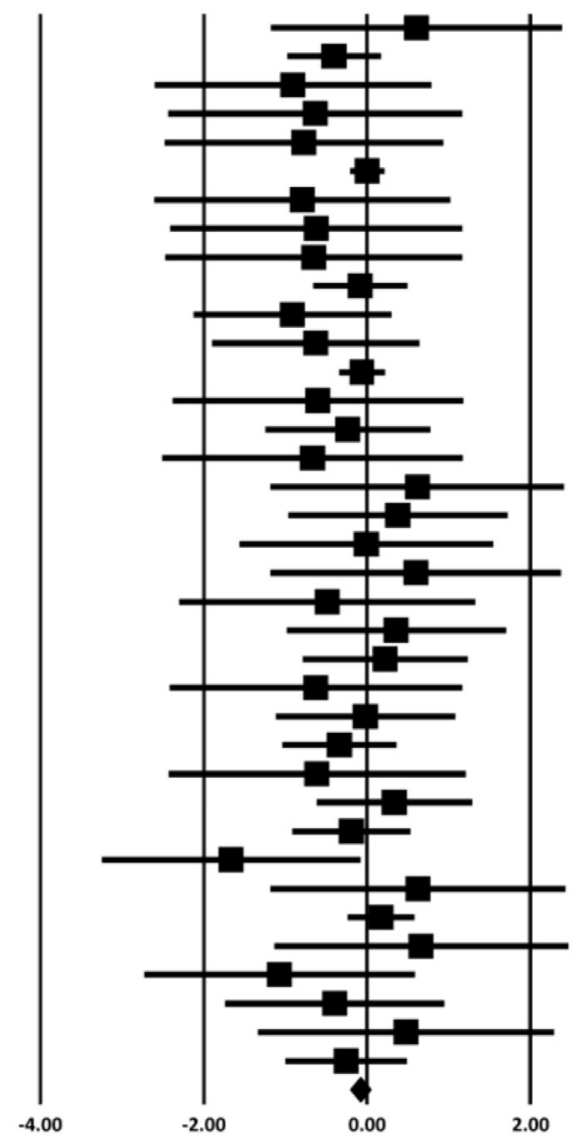

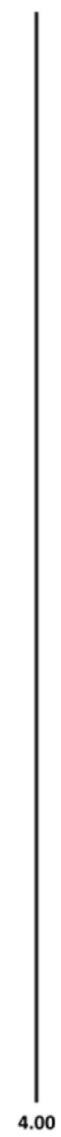

Favours OPCAB

Favours CABG

APPENDIX FIGURE 10. Sensitivity analysis of all-cause mortality as standardized difference in means. $C I$, Confidence interval; $B B S$, best bypass surgery trial; BHACAS 1, beating heart against cardioplegic arrest study 1; CORONARY, CABG off or on pump revascularization study; CRISP, The Coronary artery bypass grafting in high-RISk patients randomized to off- or on-Pump surgery; GOPCABE, German off-pump coronary artery bypass grafting in elderly patients; MASS III, off-pump and on-pump stable multivessel coronary artery bypass grafting; Octopus, comparison of on-pump and off-pump CORONARY bypass surgery in low-risk patients; PRAGUE-4, A randomized comparison between off-pump and on-pump surgery PRAGUE-4; PRAGUE-6, Off-pump versus on-pump coronary artery bypass graft surgery in patients with EuroSCORE $\geq 6 ; R O O B Y$, veterans affairs randomized on/off bypass trial; SMART, surgical management of arterial revascularization therapies; $O P C A B$, off-pump coronary artery bypass; $C A B G$, coronary artery bypass grafting. 


\begin{tabular}{|c|c|c|c|c|c|c|c|}
\hline \multirow[t]{2}{*}{ Study name } & \multicolumn{7}{|c|}{ Statistics for each study } \\
\hline & $\begin{array}{l}\text { Std diff } \\
\text { in means }\end{array}$ & $\begin{array}{c}\text { Standard } \\
\text { error }\end{array}$ & Variance & $\begin{array}{l}\text { Lower } \\
\text { limit }\end{array}$ & $\begin{array}{c}\text { Upper } \\
\text { limit }\end{array}$ & Z-Value & p-Value \\
\hline Al-Ruzzeh et al. 2006 & 0.000 & 0.784 & 0.615 & -1.537 & 1.537 & 0.000 & 1.000 \\
\hline Alwan et al. 2004 & 0.000 & 0.377 & 0.142 & -0.738 & 0.738 & 0.000 & 1.000 \\
\hline Ascione et al. 2006 & 0.633 & 0.917 & 0.841 & -1.164 & 2.431 & 0.691 & 0.490 \\
\hline Baker et al. 2001 & 0.733 & 0.926 & 0.858 & -1.082 & 2.549 & 0.792 & 0.428 \\
\hline BBS 2010 & -0.348 & 0.241 & 0.058 & -0.819 & 0.124 & -1.446 & 0.148 \\
\hline BHACAS 12002 & -0.522 & 0.468 & 0.219 & -1.440 & 0.395 & -1.116 & 0.264 \\
\hline BHACAS 22002 & -0.224 & 0.509 & 0.260 & -1.222 & 0.775 & -0.439 & 0.661 \\
\hline Carrier et al. 2003 & -0.766 & 0.865 & 0.749 & -2.462 & 0.930 & -0.886 & 0.376 \\
\hline Cavalca et al. 2006 & 0.628 & 0.914 & 0.835 & -1.163 & 2.419 & 0.687 & 0.492 \\
\hline CORONARY 2012 & -0.043 & 0.063 & 0.004 & -0.167 & 0.081 & -0.679 & 0.497 \\
\hline CRISP 2014 & 0.000 & 0.463 & 0.215 & -0.908 & 0.908 & 0.000 & 1.000 \\
\hline DOORS 2010 & 0.232 & 0.148 & 0.022 & -0.058 & 0.521 & 1.570 & 0.116 \\
\hline Gerola et al. 2004 & -0.404 & 0.400 & 0.160 & -1.188 & 0.380 & -1.010 & 0.312 \\
\hline GOPCABE 2013 & -0.060 & 0.180 & 0.033 & -0.414 & 0.293 & -0.334 & 0.738 \\
\hline Guler et al. 2001 & -1.068 & 0.914 & 0.835 & -2.860 & 0.723 & -1.169 & 0.242 \\
\hline Iqbal et al. 2014 & 0.993 & 0.431 & 0.186 & 0.147 & 1.839 & 2.302 & 0.021 \\
\hline JOCRI 2005 & 0.034 & 0.558 & 0.311 & -1.060 & 1.128 & 0.061 & 0.952 \\
\hline Khan et al. 2004 & 0.574 & 0.907 & 0.822 & -1.203 & 2.351 & 0.633 & 0.527 \\
\hline Kochamba et al. 2000 & 0.000 & 0.793 & 0.630 & -1.555 & 1.555 & 0.000 & 1.000 \\
\hline Legare et al. 2004 & 0.776 & 0.620 & 0.384 & -0.439 & 1.990 & 1.251 & 0.211 \\
\hline Lingaas et al. 2004 & 0.615 & 0.906 & 0.821 & -1.161 & 2.390 & 0.679 & 0.497 \\
\hline MASS III 2010 & -0.417 & 0.247 & 0.061 & -0.902 & 0.068 & -1.684 & 0.092 \\
\hline Michaux et al. 2011 & 0.248 & 0.529 & 0.280 & -0.790 & 1.286 & 0.468 & 0.639 \\
\hline Muneretto et al. 2003 & -0.389 & 0.680 & 0.463 & -1.722 & 0.945 & -0.571 & 0.568 \\
\hline Octopus 2003 & -0.159 & 0.286 & 0.082 & -0.720 & 0.401 & -0.557 & 0.577 \\
\hline On-Off 2012 & -0.243 & 0.360 & 0.130 & -0.949 & 0.463 & -0.674 & 0.500 \\
\hline Penttila et al. 2001 & 0.000 & 0.818 & 0.669 & -1.603 & 1.603 & 0.000 & 1.000 \\
\hline PRAGUE-11 2008 & 0.000 & 0.566 & 0.320 & -1.109 & 1.109 & 0.000 & 1.000 \\
\hline PRAGUE-4 2004 & 0.117 & 0.425 & 0.180 & -0.716 & 0.949 & 0.274 & 0.784 \\
\hline PRAGUE-6 2013 & -0.650 & 0.325 & 0.106 & -1.287 & -0.012 & -1.997 & 0.046 \\
\hline PROMISS 2010 & -0.621 & 0.643 & 0.414 & -1.881 & 0.640 & -0.965 & 0.334 \\
\hline Rastan et al. 2005 & 0.000 & 0.800 & 0.640 & -1.568 & 1.568 & 0.000 & 1.000 \\
\hline ROOBY 2009 & -0.054 & 0.168 & 0.028 & -0.383 & 0.276 & -0.320 & 0.749 \\
\hline Sahlman et al. 2003 & -1.096 & 0.849 & 0.720 & -2.759 & 0.567 & -1.291 & 0.197 \\
\hline Selvanayagam et al. 2004 & 0.000 & 0.571 & 0.326 & -1.119 & 1.119 & 0.000 & 1.000 \\
\hline SMART 2003 & -0.611 & 0.904 & 0.817 & -2.382 & 1.160 & -0.676 & 0.499 \\
\hline Smith et al. 2002 & -0.785 & 0.480 & 0.230 & -1.726 & 0.155 & -1.636 & 0.102 \\
\hline Tully et al. 2008 & 1.997 & 0.812 & 0.660 & 0.404 & 3.589 & 2.458 & 0.014 \\
\hline Unit et al. 2014 & -0.423 & 0.708 & 0.501 & -1.810 & 0.964 & -0.598 & 0.550 \\
\hline Vedin et al. 2006 & 0.685 & 0.910 & 0.828 & -1.099 & 2.468 & 0.752 & 0.452 \\
\hline Wandschneider et al. 2000 & -0.765 & 0.860 & 0.740 & -2.451 & 0.921 & -0.890 & 0.374 \\
\hline Wehlin et al. 2004 & 0.486 & 0.919 & 0.844 & -1.314 & 2.286 & 0.529 & 0.597 \\
\hline \multirow[t]{2}{*}{ Yu et al. 2014} & -0.431 & 0.357 & 0.128 & -1.131 & 0.269 & -1.207 & 0.227 \\
\hline & -0.059 & 0.044 & 0.002 & -0.145 & 0.027 & -1.349 & 0.177 \\
\hline
\end{tabular}

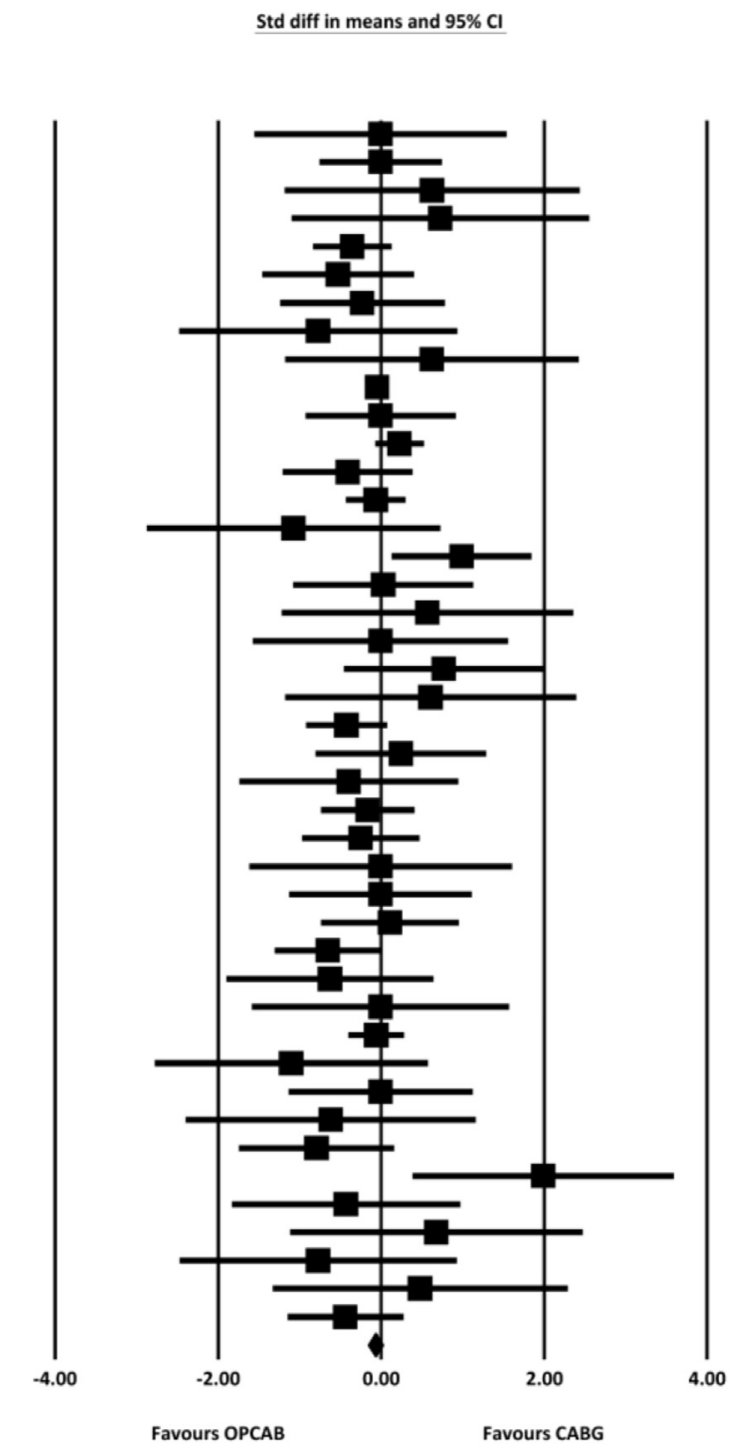

APPENDIX FIGURE 11. Sensitivity analysis of MI as standardized difference in means. $C I$, Confidence interval; $B B S$, best bypass surgery trial; $B H A C A S$ 1, beating heart against cardioplegic arrest study 1; BHACAS 2, eating heart against cardioplegic arrest study 2; CORONARY, CABG off or on pump revascularization study; CRISP, The Coronary artery bypass grafting in high-RISk patients randomized to off- or on-Pump surgery; DOORS, Danish onpump versus off-pump randomization study; GOPCABE, German off-pump coronary artery bypass grafting in elderly patients; JOCRI, Japanese off-pump coronary revascularization investigation study; MASS III, off-pump and on-pump stable multivessel coronary artery bypass grafting; Octopus, comparison of on-pump and off-pump CORONARY bypass surgery in low-risk patients; PRAGUE-11, A randomized comparison between off-pump and on-pump surgery PRAGUE-11; PRAGUE-4, A randomized comparison between off-pump and on-pump surgery PRAGUE-4; PRAGUE-6, Offpump versus on-pump coronary artery bypass graft surgery in patients with EuroSCORE $\geq 6$; PROMISS, The prospective randomized comparison of off-pump and on-pump multivessel coronary artery bypass surgery; $R O O B Y$, veterans affairs randomized on/off bypass trial; SMART, surgical management of arterial revascularization therapies; $O P C A B$, off-pump coronary artery bypass; $C A B G$, coronary artery bypass grafting. 


\begin{tabular}{|c|c|c|c|c|c|c|c|}
\hline \multirow[t]{2}{*}{ Study name } & \multicolumn{7}{|c|}{ Statistics for each study } \\
\hline & $\begin{array}{l}\text { Std diff } \\
\text { in means }\end{array}$ & $\begin{array}{c}\text { Standard } \\
\text { error }\end{array}$ & Variance & $\begin{array}{l}\text { Lower } \\
\text { limit }\end{array}$ & $\begin{array}{c}\text { Upper } \\
\text { limit }\end{array}$ & z-Value & p-Value \\
\hline Al-Ruzzeh et al. 2006 & 0.000 & 0.558 & 0.311 & -1.094 & 1.094 & 0.000 & 1.000 \\
\hline BBS 2010 & 0.045 & 0.313 & 0.098 & -0.568 & 0.658 & 0.143 & 0.887 \\
\hline BHACAS 12002 & -0.229 & 0.509 & 0.260 & -1.228 & 0.769 & -0.450 & 0.653 \\
\hline BHACAS 22002 & -0.611 & 0.641 & 0.411 & -1.869 & 0.646 & -0.953 & 0.341 \\
\hline Bicer M et al. 2014 & -1.142 & 0.849 & 0.720 & -2.805 & 0.521 & -1.345 & 0.178 \\
\hline Carrier et al. 2003 & -0.469 & 0.911 & 0.830 & -2.254 & 1.316 & -0.515 & 0.606 \\
\hline CORONARY 2012 & -0.065 & 0.156 & 0.024 & -0.370 & 0.240 & -0.419 & 0.675 \\
\hline CRISP 2014 & 0.393 & 0.684 & 0.468 & -0.948 & 1.733 & 0.574 & 0.566 \\
\hline Diegeler et al. 2000 & -0.633 & 0.917 & 0.841 & -2.431 & 1.164 & -0.691 & 0.490 \\
\hline DOORS 2010 & -0.334 & 0.221 & 0.049 & -0.767 & 0.098 & -1.515 & 0.130 \\
\hline GOPCABE 2013 & -0.118 & 0.147 & 0.022 & -0.407 & 0.170 & -0.805 & 0.421 \\
\hline Hernandez et al. 2007 & -1.073 & 0.837 & 0.701 & -2.714 & 0.568 & -1.281 & 0.200 \\
\hline Iqbal et al. 2014 & -0.629 & 0.457 & 0.209 & -1.525 & 0.267 & -1.375 & 0.169 \\
\hline Jares et al. 2006 & -0.661 & 0.934 & 0.872 & -2.491 & 1.169 & -0.708 & 0.479 \\
\hline JOCRI 2005 & -0.579 & 0.904 & 0.818 & -2.352 & 1.193 & -0.641 & 0.522 \\
\hline Jongman et al. 2014 & -0.624 & 0.912 & 0.831 & -2.411 & 1.162 & -0.685 & 0.494 \\
\hline Kok et al. 2014 & -0.624 & 0.912 & 0.831 & -2.411 & 1.162 & -0.685 & 0.494 \\
\hline Lee et al. 2003 & -0.624 & 0.912 & 0.831 & -2.411 & 1.162 & -0.685 & 0.494 \\
\hline Legare et al. 2004 & 0.895 & 0.856 & 0.734 & -0.784 & 2.573 & 1.045 & 0.296 \\
\hline Lingaas et al. 2004 & -0.906 & 0.860 & 0.740 & -2.592 & 0.780 & -1.053 & 0.292 \\
\hline Lund et al. 2003 & -0.755 & 0.913 & 0.834 & -2.545 & 1.035 & -0.827 & 0.408 \\
\hline MASS III 2010 & -0.393 & 0.482 & 0.232 & -1.337 & 0.551 & -0.816 & 0.415 \\
\hline Medved et al. 2008 & -0.401 & 0.691 & 0.477 & -1.756 & 0.953 & -0.581 & 0.561 \\
\hline Michaux et al. 2011 & 0.628 & 0.914 & 0.835 & -1.163 & 2.419 & 0.687 & 0.492 \\
\hline Motallebzadeh et al. 2004 & -0.479 & 0.920 & 0.846 & -2.282 & 1.323 & -0.521 & 0.602 \\
\hline Motallebzadeh et al. 2007 & -0.638 & 0.641 & 0.411 & -1.894 & 0.619 & -0.994 & 0.320 \\
\hline Muneretto et al. 2003 & -0.900 & 0.858 & 0.736 & -2.582 & 0.782 & -1.049 & 0.294 \\
\hline Nesher et al. 2006 & -0.205 & 0.513 & 0.263 & -1.211 & 0.801 & -0.400 & 0.689 \\
\hline Niranjan et al. 2006 & 0.000 & 0.790 & 0.624 & -1.548 & 1.548 & 0.000 & 1.000 \\
\hline Octopus 2003 & -0.398 & 0.678 & 0.460 & -1.728 & 0.932 & -0.587 & 0.557 \\
\hline On-Off 2012 & -0.622 & 0.902 & 0.814 & -2.390 & 1.146 & -0.689 & 0.491 \\
\hline PRAGUE-4 2004 & -0.937 & 0.856 & 0.733 & -2.615 & 0.740 & -1.095 & 0.274 \\
\hline PRAGUE-6 2013 & -0.179 & 0.509 & 0.259 & -1.178 & 0.819 & -0.352 & 0.725 \\
\hline PROMISS 2010 & -0.902 & 0.859 & 0.738 & -2.586 & 0.781 & -1.050 & 0.294 \\
\hline ROOBY 2009 & 0.309 & 0.245 & 0.060 & -0.172 & 0.790 & 1.259 & 0.208 \\
\hline Sahlman et al. 2003 & 0.046 & 0.796 & 0.633 & -1.514 & 1.606 & 0.058 & 0.954 \\
\hline Sajja et al. 2007 & -0.577 & 0.906 & 0.821 & -2.353 & 1.199 & -0.637 & 0.524 \\
\hline Selvanayagam et al. 2004 & -0.624 & 0.912 & 0.831 & -2.411 & 1.162 & -0.685 & 0.494 \\
\hline SMART 2003 & 0.000 & 0.557 & 0.310 & -1.092 & 1.092 & 0.000 & 1.000 \\
\hline Yu et al. 2014 & -1.378 & 0.822 & 0.676 & -2.989 & 0.233 & -1.677 & 0.094 \\
\hline & -0.183 & 0.070 & 0.005 & -0.321 & -0.046 & -2.610 & 0.009 \\
\hline
\end{tabular}

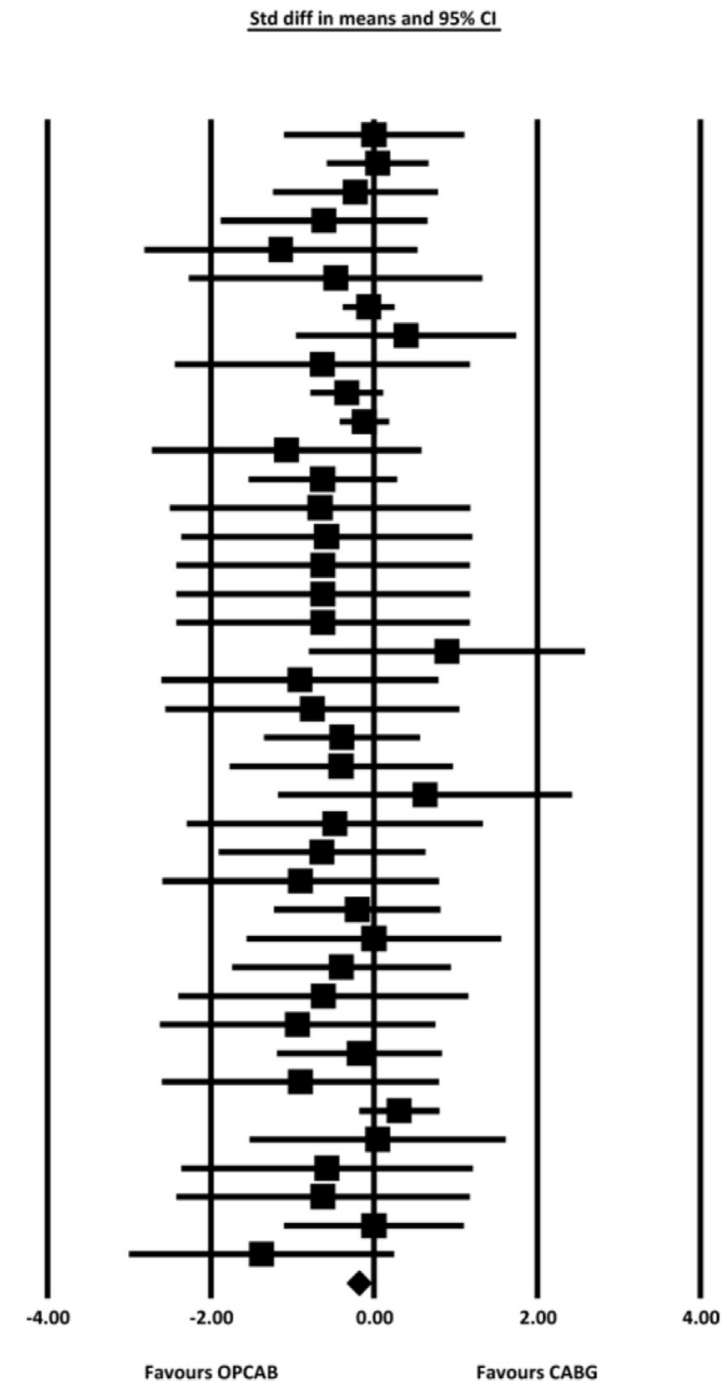

APPENDIX FIGURE 12. Sensitivity analysis of cerebral stroke as standardized difference in means. $C I$, Confidence interval; $B B S$, best bypass surgery trial; BHACAS 1, beating heart against cardioplegic arrest study 1; BHACAS 2, eating heart against cardioplegic arrest study 2; CORONARY, CABG off or on pump revascularization study; CRISP, The Coronary artery bypass grafting in high-RISk patients randomized to off- or on-Pump surgery; DOORS, Danish on-pump versus off-pump randomization study; GOPCABE, German off-pump coronary artery bypass grafting in elderly patients; JOCRI, Japanese off-pump coronary revascularization investigation study; MASS III, off-pump and on-pump stable multivessel coronary artery bypass grafting; Octopus, comparison of on-pump and off-pump CORONARY bypass surgery in low-risk patients; PRAGUE-11, A randomized comparison between off-pump and on-pump surgery PRAGUE-11; PRAGUE-4, A randomized comparison between off-pump and on-pump surgery PRAGUE-4; PRAGUE-6, Offpump versus on-pump coronary artery bypass graft surgery in patients with EuroSCORE $\geq 6$; PROMISS, The prospective randomized comparison of off-pump and on-pump multivessel coronary artery bypass surgery; ROOBY, veterans affairs randomized on/off bypass trial; SMART, surgical management of arterial revascularization therapies; $O P C A B$, off-pump coronary artery bypass; $C A B G$, coronary artery bypass grafting. 


\begin{tabular}{|c|c|c|c|c|c|c|c|}
\hline Study or Subgroup & Risk Difference & & CAB & CABG & Wrimbt. & Risk Difference & $\begin{array}{c}\text { Risk Difference } \\
\text { IV, Random, } 95 \% \mathrm{CI}\end{array}$ \\
\hline Al-Ruzzeh et al. 2006 & 0.011905 & 0.016516 & 84 & 84 & $1.8 \%$ & $0.01[-0.02,0.04]$ & † \\
\hline BBS 2010 & -0.03318 & 0.023805 & 177 & 164 & $0.9 \%$ & $-0.03[-0.08,0.01]$ & \\
\hline BHACAS 12002 & -0.02 & 0.016965 & 100 & 100 & $1.7 \%$ & $-0.02[-0.05,0.01]$ & \\
\hline Bicer M et al. 2014 & -0.04001 & 0.053094 & 25 & 25 & $0.2 \%$ & $-0.04[-0.14,0.06]$ & \\
\hline Carrier et al. 2003 & 0 & 0.046939 & 32 & 33 & $0.2 \%$ & $0.00[-0.09,0.09]$ & \\
\hline CORONARY 2012 & 0.000442 & 0.004533 & 2375 & 2377 & $24.0 \%$ & $0.00[-0.01,0.01]$ & \\
\hline Covino et al. 2001 & -0.06254 & 0.075848 & 21 & 16 & $0.1 \%$ & $-0.06[-0.21,0.09]$ & \\
\hline CRISP 2014 & -0.01887 & 0.025887 & 53 & 53 & $0.7 \%$ & $-0.02[-0.07,0.03]$ & \\
\hline Czerny et al. 2000 & -0.06672 & 0.084968 & 15 & 15 & $0.1 \%$ & $-0.07[-0.23,0.10]$ & \\
\hline DOORS 2010 & -0.00222 & 0.008534 & 450 & 450 & $6.8 \%$ & $-0.00[-0.02,0.01]$ & \\
\hline Fattouch et al. 2009 & -0.06109 & 0.036619 & 63 & 65 & $0.4 \%$ & $-0.06[-0.13,0.01]$ & \\
\hline Gerola et al. 2004 & -0.025 & 0.024609 & 80 & 80 & $0.8 \%$ & $-0.03[-0.07,0.02]$ & \\
\hline GOPCABE 2013 & -0.00242 & 0.006269 & 1271 & 1268 & $12.6 \%$ & $-0.00[-0.01,0.01]$ & \\
\hline Hernandez et al. 2007 & -0.0098 & 0.013751 & 99 & 102 & $2.6 \%$ & $-0.01[-0.04,0.02]$ & \\
\hline lqbal et al. 2014 & -0.01 & 0.02207 & 100 & 100 & $1.0 \%$ & $-0.01[-0.05,0.03]$ & \\
\hline Jares et al. 2006 & -0.10017 & 0.121337 & 10 & 10 & $0.0 \%$ & $-0.10[-0.34,0.14]$ & \\
\hline Lee et al. 2003 & 0.03334 & 0.044705 & 30 & 30 & $0.2 \%$ & $0.03[-0.05,0.12]$ & \\
\hline Legare et al. 2004 & 0.006667 & 0.011483 & 150 & 150 & $3.7 \%$ & $0.01[-0.02,0.03]$ & - \\
\hline Lingaas et al. 2004 & 0 & 0.023375 & 60 & 60 & $0.9 \%$ & $0.00[-0.05,0.05]$ & \\
\hline MASS III 2010 & 0.00641 & 0.008986 & 156 & 155 & $6.1 \%$ & $0.01[-0.01,0.02]$ & r \\
\hline Motallebzadeh et al. 2004 & -0.05002 & 0.071127 & 15 & 20 & $0.1 \%$ & $-0.05[-0.19,0.09]$ & \\
\hline Motallebzadeh et al. 2007 & 0.008903 & 0.016121 & 108 & 104 & $1.9 \%$ & $0.01[-0.02,0.04]$ & \\
\hline Muneretto et al. 2003 & 0.011364 & 0.025034 & 88 & 88 & $0.8 \%$ & $0.01[-0.04,0.06]$ & \\
\hline Niranjan et al. 2006 & -0.025 & 0.03397 & 40 & 40 & $0.4 \%$ & $-0.03[-0.09,0.04]$ & \\
\hline Octopus 2003 & -0.0003 & 0.014136 & 142 & 139 & $2.5 \%$ & $-0.00[-0.03,0.03]$ & \\
\hline On-Off 2012 & -0.01525 & 0.01596 & 208 & 203 & $1.9 \%$ & $-0.02[-0.05,0.02]$ & \\
\hline Paparella et al. 2006 & -0.06254 & 0.081481 & 16 & 16 & $0.1 \%$ & $-0.06[-0.22,0.10]$ & \\
\hline PRAGUE-4 2004 & 0.008814 & 0.012016 & 208 & 192 & $3.4 \%$ & $0.01[-0.01,0.03]$ & \\
\hline PRAGUE-6 2013 & -0.01526 & 0.029907 & 98 & 107 & $0.6 \%$ & $-0.02[-0.07,0.04]$ & \\
\hline Raja et al. 2003 & -0.06004 & 0.020306 & 150 & 150 & $1.2 \%$ & $-0.06[-0.10,-0.02]$ & \\
\hline Rastan et al. 2005 & 0.050021 & 0.065355 & 20 & 20 & $0.1 \%$ & $0.05[-0.08,0.18]$ & \\
\hline ROOBY 2009 & 0.004475 & 0.005016 & 1104 & 1099 & $19.6 \%$ & $0.00[-0.01,0.01]$ & 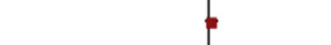 \\
\hline Sahlman et al. 2003 & 0.041679 & 0.054148 & 24 & 26 & $0.2 \%$ & $0.04[-0.06,0.15]$ & \\
\hline Sajja et al. 2007 & -0.05002 & 0.032242 & 56 & 60 & $0.5 \%$ & $-0.05[-0.11,0.01]$ & \\
\hline SMART 2003 & -0.01 & 0.017176 & 100 & 100 & $1.7 \%$ & $-0.01[-0.04,0.02]$ & \\
\hline Wehlin et al. 2004 & 0.047637 & 0.067632 & 21 & 16 & $0.1 \%$ & $0.05[-0.08,0.18]$ & \\
\hline Yu et al. 2014 & -0.03923 & 0.058793 & 51 & 51 & $0.1 \%$ & $-0.04[-0.15,0.08]$ & \\
\hline Total $(95 \% \mathrm{Cl})$ & & & 7800 & 7768 & $100.0 \%$ & $-0.00[-0.01,0.00]$ & \\
\hline \multicolumn{7}{|c|}{$\begin{array}{l}\text { Heterogeneity: } \text { Tau }^{2}=0.00 ; \mathrm{Chi}^{2}=30.98, \mathrm{df}=36(P=0.71) ; \mathrm{I}^{2}=0 \% \\
\text { Test for overall effect: } Z=0.59(P=0.55)\end{array}$} & $\begin{array}{cccc}-0.2 & -0.1 & 0 & 0.1 \\
\text { Favours } & \text { OPCAB } & \text { Favours }\end{array}$ \\
\hline
\end{tabular}

APPENDIX FIGURE 13. Sensitivity analysis of all-cause mortality after arcsine transformation of risk difference. $O P C A B$, Off-pump coronary artery bypass; $C A B G$, coronary artery bypass grafting; $S E$, standard error; $C I$, confidence interval; $B B S$, best bypass surgery trial; $B H A C A S 1$, beating heart against cardioplegic arrest study 1; CORONARY, CABG off or on pump revascularization study; CRISP, The Coronary artery bypass grafting in high-RISk patients randomized to off- or on-Pump surgery; DOORS, Danish on-pump versus off-pump randomization study; GOPCABE, German off-pump coronary artery bypass grafting in elderly patients; MASS III, off-pump and on-pump stable multivessel coronary artery bypass grafting; Octopus, comparison of on-pump and off-pump CORONARY bypass surgery in low-risk patients; PRAGUE-4, A randomized comparison between off-pump and on-pump surgery PRAGUE4; PRAGUE-6, Off-pump versus on-pump coronary artery bypass graft surgery in patients with EuroSCORE $\geq 6$; PROMISS, The prospective randomized comparison of off-pump and on-pump multivessel coronary artery bypass surgery; ROOBY, veterans affairs randomized on/off bypass trial; SMART, surgical management of arterial revascularization therapies; $d f$, degrees of freedom. 


\begin{tabular}{|c|c|c|c|c|c|c|c|}
\hline Study or Subgroup & Risk Difference & \multicolumn{2}{|c|}{ OPCAB } & \multicolumn{2}{|l|}{ CABG } & $\begin{array}{l}\text { Risk Difference } \\
\text { IV, Random, } 95 \% \mathrm{CI}\end{array}$ & $\begin{array}{c}\text { Risk Difference } \\
\text { IV, Random, } 95 \% \text { C }\end{array}$ \\
\hline Al-Ruzzeh et al. 2006 & 0 & 0.016736 & 84 & 84 & $4.2 \%$ & $0.00[-0.03,0.03]$ & + \\
\hline Alwan et al. 2004 & 0 & 0.083746 & 35 & 35 & $0.2 \%$ & $0.00[-0.16,0.16]$ & \\
\hline Ascione et al. 2006 & 0.050021 & 0.065355 & 20 & 20 & $0.4 \%$ & $0.05[-0.08,0.18]$ & \\
\hline Baker et al. 2001 & 0.08343 & 0.100166 & 12 & 14 & $0.2 \%$ & $0.08[-0.11,0.28]$ & \\
\hline BBS 2010 & -0.04063 & 0.027921 & 177 & 164 & $1.9 \%$ & $-0.04[-0.10,0.01]$ & \\
\hline BHACAS 12002 & -0.03 & 0.025907 & 100 & 100 & $2.1 \%$ & $-0.03[-0.08,0.02]$ & \\
\hline BHACAS 22002 & -0.0097 & 0.021942 & 100 & 101 & $2.8 \%$ & $-0.01[-0.05,0.03]$ & \\
\hline Carrier et al. 2003 & 0 & 0.046939 & 32 & 33 & $0.7 \%$ & $0.00[-0.09,0.09]$ & \\
\hline Cavalca et al. 2006 & 0.040011 & 0.053094 & 25 & 25 & $0.6 \%$ & $0.04[-0.06,0.14]$ & \\
\hline CORONARY 2012 & -0.00499 & 0.007354 & 2375 & 2377 & $10.0 \%$ & $-0.00[-0.02,0.01]$ & \\
\hline CRISP 2014 & 0 & 0.044905 & 53 & 53 & $0.8 \%$ & $0.00[-0.09,0.09]$ & \\
\hline DOORS 2010 & 0.02667 & 0.016862 & 450 & 450 & $4.2 \%$ & $0.03[-0.01,0.06]$ & - \\
\hline Gerola et al. 2004 & -0.03751 & 0.036317 & 80 & 80 & $1.2 \%$ & $-0.04[-0.11,0.03]$ & \\
\hline GOPCABE 2013 & -0.00161 & 0.00482 & 1271 & 1268 & $12.2 \%$ & $-0.00[-0.01,0.01]$ & \\
\hline Guler et al. 2001 & -0.05558 & 0.064238 & 40 & 18 & $0.4 \%$ & $-0.06[-0.18,0.07]$ & \\
\hline Iqbal et al. 2014 & 0.090122 & 0.034285 & 100 & 100 & $1.3 \%$ & $0.09[0.02,0.16]$ & \\
\hline JOCRI 2005 & 0.001436 & 0.023697 & 81 & 86 & $2.5 \%$ & $0.00[-0.05,0.05]$ & - \\
\hline Khan et al. 2004 & 0.01852 & 0.025939 & 54 & 50 & $2.1 \%$ & $0.02[-0.03,0.07]$ & \\
\hline Kochamba et al. 2000 & 0 & 0.047936 & 29 & 29 & $0.7 \%$ & $0.00[-0.09,0.09]$ & \\
\hline Legare et al. 2004 & 0.020001 & 0.014738 & 150 & 150 & $5.1 \%$ & $0.02[-0.01,0.05]$ & - \\
\hline Lingaas et al. 2004 & 0.016667 & 0.022947 & 60 & 60 & $2.6 \%$ & $0.02[-0.03,0.06]$ & \\
\hline MASS III 2010 & -0.05197 & 0.030156 & 156 & 155 & $1.6 \%$ & $-0.05[-0.11,0.01]$ & \\
\hline Michaux et al. 2011 & 0.040011 & 0.084766 & 25 & 25 & $0.2 \%$ & $0.04[-0.13,0.21]$ & \\
\hline Muneretto et al. 2003 & -0.01136 & 0.019496 & 88 & 88 & $3.4 \%$ & $-0.01[-0.05,0.03]$ & \\
\hline Octopus 2003 & -0.01545 & 0.027675 & 142 & 139 & $1.9 \%$ & $-0.02[-0.07,0.04]$ & \\
\hline On-Off 2012 & -0.01033 & 0.015231 & 208 & 203 & $4.8 \%$ & $-0.01[-0.04,0.02]$ & \\
\hline Penttila et al. 2001 & 0 & 0.122891 & 11 & 11 & $0.1 \%$ & $0.00[-0.24,0.24]$ & \\
\hline PRAGUE-11 2008 & 0 & 0.048753 & 40 & 40 & $0.7 \%$ & $0.00[-0.10,0.10]$ & \\
\hline PRAGUE-4 2004 & 0.003606 & 0.013069 & 208 & 192 & $5.9 \%$ & $0.00[-0.02,0.03]$ & \\
\hline PRAGUE-6 2013 & -0.08077 & 0.037385 & 98 & 107 & $1.1 \%$ & $-0.08[-0.15,-0.01]$ & \\
\hline PROMISS 2010 & -0.02667 & 0.026221 & 75 & 75 & $2.1 \%$ & $-0.03[-0.08,0.02]$ & \\
\hline Rastan et al. 2005 & 0 & 0.068975 & 20 & 20 & $0.3 \%$ & $0.00[-0.14,0.14]$ & \\
\hline ROOBY 2009 & -0.00191 & 0.005962 & 1104 & 1099 & $11.2 \%$ & $-0.00[-0.01,0.01]$ & \\
\hline Sahlman et al. 2003 & -0.11564 & 0.070505 & 24 & 26 & $0.3 \%$ & $-0.12[-0.25,0.02]$ & \\
\hline Selvanayagam et al. 2004 & 0 & 0.064451 & 30 & 30 & $0.4 \%$ & $0.00[-0.13,0.13]$ & \\
\hline SMART 2003 & -0.01 & 0.013916 & 100 & 100 & $5.4 \%$ & $-0.01[-0.04,0.02]$ & \\
\hline Smith et al. 2002 & -0.21066 & 0.11562 & 21 & 23 & $0.1 \%$ & $-0.21[-0.44,0.02]$ & \\
\hline Tully et al. 2008 & 0.339837 & 0.087206 & 30 & 36 & $0.2 \%$ & $0.34[0.17,0.51]$ & \\
\hline Unić et al. 2014 & -0.06672 & 0.109082 & 15 & 15 & $0.1 \%$ & $-0.07[-0.28,0.15]$ & \\
\hline Vedin et al. 2006 & 0.030308 & 0.039785 & 33 & 37 & $1.0 \%$ & $0.03[-0.05,0.11]$ & \\
\hline Wandschneider et al. 2000 & -0.02986 & 0.026406 & 52 & 67 & $2.1 \%$ & $-0.03[-0.08,0.02]$ & \\
\hline Wehlin et al. 2004 & 0.047637 & 0.067632 & 21 & 16 & $0.4 \%$ & $0.05[-0.08,0.18]$ & \\
\hline Yu et al. 2014 & -0.07851 & 0.063371 & 51 & 51 & $0.4 \%$ & $-0.08[-0.20,0.05]$ & \\
\hline Total $(95 \% \mathrm{Cl})$ & & & 7880 & 7852 & $100.0 \%$ & $-0.00[-0.01,0.01]$ & \\
\hline $\begin{array}{l}\text { Heterogeneity: } \mathrm{Tau}^{2}=0.00 \text {; } \\
\text { Test for overall effect: } Z=0\end{array}$ & $\begin{array}{l}C h i^{2}=55.62, d f=42 \\
66(P=0.51)\end{array}$ & $2(P=0.08$ & $=24 \%$ & & & & 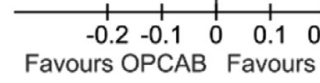 \\
\hline
\end{tabular}

APPENDIX FIGURE 14. Sensitivity analysis of MI after arcsine transformation of risk difference. $O P C A B$, Off-pump coronary artery bypass; $C A B G$, coronary artery bypass grafting; $S E$, standard error; $C I$, confidence interval; $B B S$, best bypass surgery trial; BHACAS 1 , beating heart against cardioplegic arrest study 1; BHACAS 2, eating heart against cardioplegic arrest study 2; CORONARY, CABG off or on pump revascularization study; CRISP, The Coronary artery bypass grafting in high-RISk patients randomized to off- or on-Pump surgery; DOORS, Danish on-pump versus off-pump randomization study; GOPCABE, German off-pump coronary artery bypass grafting in elderly patients; JOCRI, Japanese off-pump coronary revascularization investigation study; MASS III, off-pump and on-pump stable multivessel coronary artery bypass grafting; Octopus, comparison of on-pump and off-pump CORONARY bypass surgery in low-risk patients; PRAGUE-11, A randomized comparison between off-pump and on-pump surgery PRAGUE-11; PRAGUE-4, A randomized comparison between off-pump and on-pump surgery PRAGUE-4; PRAGUE-6, Off-pump versus on-pump coronary artery bypass graft surgery in patients with EuroSCORE $\geq 6$; PROMISS, The prospective randomized comparison of off-pump and on-pump multivessel coronary artery bypass surgery; $R O O B Y$, veterans affairs randomized on/off bypass trial; SMART, surgical management of arterial revascularization therapies; $d f$, degrees of freedom. 


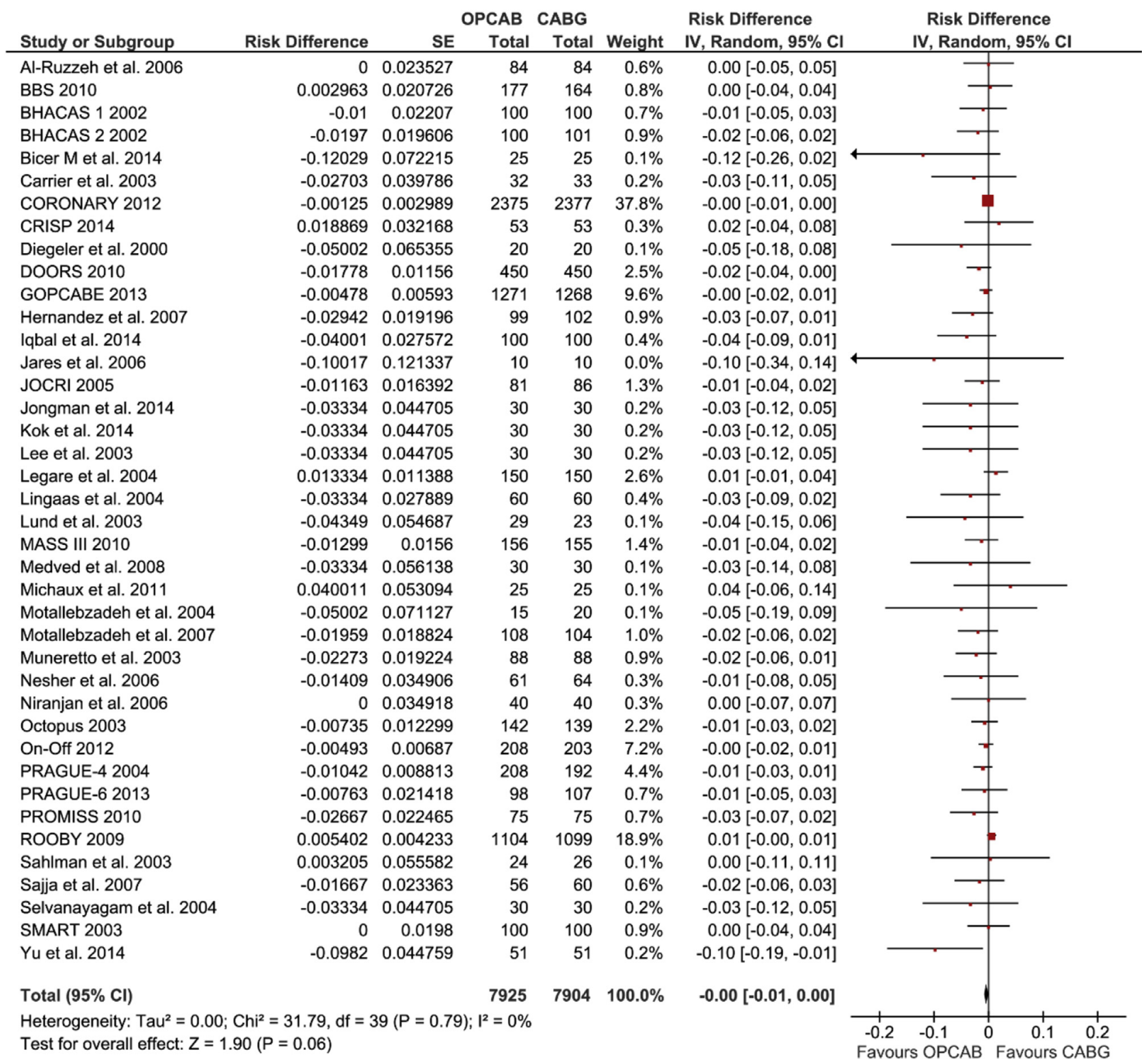

APPENDIX FIGURE 15. Sensitivity analysis of cerebral stroke after arcsine transformation of risk difference. $O P C A B$, Off-pump coronary artery bypass; $C A B G$, coronary artery bypass grafting; $S E$, standard error; $C I$, confidence interval; $B B S$, best bypass surgery trial; BHACAS 1 , beating heart against cardioplegic arrest study 1; BHACAS 2, eating heart against cardioplegic arrest study 2; CORONARY, CABG off or on pump revascularization study; CRISP, The Coronary artery bypass grafting in high-RISk patients randomized to off- or on-Pump surgery; DOORS, Danish on-pump versus off-pump randomization study; GOPCABE, German off-pump coronary artery bypass grafting in elderly patients; JOCRI, Japanese off-pump coronary revascularization investigation study; MASS III, off-pump and on-pump stable multivessel coronary artery bypass grafting; Octopus, comparison of on-pump and offpump CORONARY bypass surgery in low-risk patients; PRAGUE-4, A randomized comparison between off-pump and on-pump surgery PRAGUE-4; PRAGUE-6, Off-pump versus on-pump coronary artery bypass graft surgery in patients with EuroSCORE $\geq 6$; PROMISS, The prospective randomized comparison of off-pump and on-pump multivessel coronary artery bypass surgery; ROOBY, veterans affairs randomized on/off bypass trial; SMART, surgical management of arterial revascularization therapies; $d f$, degrees of freedom. 
APPENDIX TABLE 1. PRISMA checklist

\begin{tabular}{|c|c|c|c|}
\hline Section/topic & \# & Checklist item & Reported on page \# \\
\hline \multicolumn{4}{|l|}{ Title } \\
\hline Title & 1 & Identify the report as a systematic review, meta-analysis, or both. & 1 \\
\hline \multicolumn{4}{|l|}{ Abstract } \\
\hline Structured summary & 2 & $\begin{array}{l}\text { Provide a structured summary including, as applicable: background; } \\
\text { objectives; data sources; study eligibility criteria, participants, and } \\
\text { interventions; study appraisal and synthesis methods; results; limitations; } \\
\text { conclusions and implications of key findings; systematic review } \\
\text { registration number. }\end{array}$ & 3 \\
\hline \multicolumn{4}{|l|}{ Introduction } \\
\hline Rationale & 3 & Describe the rationale for the review in the context of what is already known. & 5 \\
\hline Objectives & 4 & $\begin{array}{l}\text { Provide an explicit statement of questions being addressed with reference to } \\
\text { participants, interventions, comparisons, outcomes, and study design } \\
\text { (PICOS). }\end{array}$ & 5 \\
\hline \multicolumn{4}{|l|}{ Methods } \\
\hline Protocol and registration & 5 & $\begin{array}{l}\text { Indicate if a review protocol exists, if and where it can be accessed (eg, Web } \\
\text { address), and, if available, provide registration information including } \\
\text { registration number. }\end{array}$ & NA \\
\hline Eligibility criteria & 6 & $\begin{array}{l}\text { Specify study characteristics (eg, PICOS, length of follow-up) and report } \\
\text { characteristics (eg, years considered, language, publication status) } \\
\text { used as criteria for eligibility, giving rationale. }\end{array}$ & 6,7 \\
\hline Information sources & 7 & $\begin{array}{l}\text { Describe all information sources (eg, databases with dates of coverage, } \\
\text { contact with study authors to identify additional studies) in the search } \\
\text { and date last searched. }\end{array}$ & 6 \\
\hline Search & 8 & $\begin{array}{l}\text { Present full electronic search strategy for at least one database, including } \\
\text { any limits used, such that it could be repeated. }\end{array}$ & 6 \\
\hline Study selection & 9 & $\begin{array}{l}\text { State the process for selecting studies (ie, screening, eligibility, included in } \\
\text { systematic review, and, if applicable, included in the meta-analysis). }\end{array}$ & 6,7 \\
\hline Data collection process & 10 & $\begin{array}{l}\text { Describe method of data extraction from reports (eg, piloted forms, } \\
\text { independently, in duplicate) and any processes for obtaining and } \\
\text { confirming data from investigators. }\end{array}$ & 6,7 \\
\hline Data items & 11 & $\begin{array}{l}\text { List and define all variables for which data were sought (eg, PICOS, funding } \\
\text { sources) and any assumptions and simplifications made. }\end{array}$ & 7 \\
\hline Risk of bias in individual studies & 12 & $\begin{array}{l}\text { Describe methods used for assessing risk of bias of individual studies } \\
\text { (including specification of whether this was done at the study or } \\
\text { outcome level), and how this information is to be used in any data } \\
\text { synthesis. }\end{array}$ & 7 \\
\hline Summary measures & 13 & State the principal summary measures (eg, risk ratio, difference in means). & 7,8 \\
\hline Synthesis of results & 14 & $\begin{array}{l}\text { Describe the methods of handling data and combining results of studies, if } \\
\text { done, including measures of consistency }\left(\mathrm{eg}, I^{2}\right) \text { for each meta- } \\
\text { analysis. }\end{array}$ & 7,8 \\
\hline Risk of bias across studies & 15 & $\begin{array}{l}\text { Specify any assessment of risk of bias that may affect the cumulative } \\
\text { evidence (eg, publication bias, selective reporting within studies). }\end{array}$ & Table 1, Appendix Table 4 \\
\hline Additional analyses & 16 & $\begin{array}{l}\text { Describe methods of additional analyses (eg, sensitivity or subgroup } \\
\text { analyses, meta-regression), if done, indicating which were prespecified. }\end{array}$ & 7,8 \\
\hline \multicolumn{4}{|l|}{ Results } \\
\hline Study selection & 17 & $\begin{array}{l}\text { Give numbers of studies screened, assessed for eligibility, and included in } \\
\text { the review, with reasons for exclusions at each stage, ideally with a flow } \\
\text { diagram. }\end{array}$ & 9 \\
\hline Study characteristics & 18 & $\begin{array}{l}\text { For each study, present characteristics for which data were extracted (eg, } \\
\text { study size, PICOS, follow-up period) and provide the citations. }\end{array}$ & Table 1 Appendix Table 3 \\
\hline Risk of bias within studies & 19 & $\begin{array}{l}\text { Present data on risk of bias of each study and, if available, any outcome level } \\
\text { assessment (see item 12). }\end{array}$ & 9, Table 1, Appendix Table 4 \\
\hline Results of individual studies & 20 & $\begin{array}{l}\text { For all outcomes considered (benefits or harms), present, for each study: (a) } \\
\text { simple summary data for each intervention group (b) effect estimates } \\
\text { and confidence intervals, ideally with a forest plot. }\end{array}$ & $9-11$ \\
\hline
\end{tabular}


APPENDIX TABLE 1. Continued

\begin{tabular}{|c|c|c|c|}
\hline Section/topic & \# & Checklist item & Reported on page \# \\
\hline Synthesis of results & 21 & $\begin{array}{l}\text { Present results of each meta-analysis done, including confidence intervals } \\
\text { and measures of consistency. }\end{array}$ & $9-11$ \\
\hline Risk of bias across studies & 22 & Present results of any assessment of risk of bias across studies (see Item 15). & 9, Table 1, Appendix Table 4 \\
\hline Additional analysis & 23 & $\begin{array}{l}\text { Give results of additional analyses, if done (eg, sensitivity or subgroup } \\
\text { analyses, meta-regression [see Item 16]). }\end{array}$ & 11 \\
\hline \multicolumn{4}{|l|}{ Discussion } \\
\hline Summary of evidence & 24 & $\begin{array}{l}\text { Summarize the main findings including the strength of evidence for each } \\
\text { main outcome; consider their relevance to key groups (eg, healthcare } \\
\text { providers, users, and policy makers). }\end{array}$ & $11-17$ \\
\hline Limitations & 25 & $\begin{array}{l}\text { Discuss limitations at study and outcome level (eg, risk of bias), and at } \\
\text { review-level (eg, incomplete retrieval of identified research, reporting } \\
\text { bias). }\end{array}$ & $11-17$ \\
\hline Conclusions & 26 & $\begin{array}{l}\text { Provide a general interpretation of the results in the context of other } \\
\text { evidence, and implications for future research. }\end{array}$ & 17 \\
\hline \multicolumn{4}{|l|}{ Funding } \\
\hline Funding & 27 & $\begin{array}{l}\text { Describe sources of funding for the systematic review and other support (eg, } \\
\text { supply of data); role of funders for the systematic review. }\end{array}$ & 17 , NA \\
\hline
\end{tabular}


APPENDIX TABLE 2. Medline search strategy

\begin{tabular}{|c|c|c|}
\hline Search & Query & Items found \\
\hline 1 & OPCAB AND trial & 279 \\
\hline 2 & Beating heart AND trial & 307 \\
\hline 3 & OPCABG AND trial & 32 \\
\hline 4 & Off-Pump AND trial & 921 \\
\hline 5 & Without cardiopulmonary AND trial & 8248 \\
\hline 6 & Without extracorporeal circulation AND trial & 3216 \\
\hline 7 & OPCAB AND study & 768 \\
\hline 8 & Beating heart AND study & 3339 \\
\hline 9 & OPCABG AND study & 104 \\
\hline 10 & Off-Pump AND study & 2481 \\
\hline 11 & Without cardiopulmonary AND study & 27,313 \\
\hline 12 & Without extracorporeal circulation AND study & 10,932 \\
\hline 13 & OPCAB AND random* & 261 \\
\hline 14 & Beating heart AND random* & 316 \\
\hline 15 & OPCABG AND random* & 35 \\
\hline 16 & Off-Pump AND random* & 831 \\
\hline 17 & Without cardiopulmonary AND random* & 8057 \\
\hline 18 & Without extracorporeal circulation AND random* & 2417 \\
\hline 19 & ONCAB AND trial & 21 \\
\hline 20 & CABG AND trial & 3043 \\
\hline 21 & On-pump AND trial & 533 \\
\hline 22 & Cardiopulmonary bypass AND trial & 4889 \\
\hline 23 & Extracorporeal circulation AND trial & 6333 \\
\hline 24 & Conventional bypass AND trial & 1038 \\
\hline 25 & ONCAB AND study & 62 \\
\hline 26 & CABG AND study & 8552 \\
\hline 27 & On-pump AND study & 1312 \\
\hline 28 & Cardiopulmonary bypass AND study & 13,316 \\
\hline 29 & Extracorporeal circulation AND study & 19,657 \\
\hline 30 & Conventional bypass AND study & 2615 \\
\hline 31 & ONCAB AND random* & 29 \\
\hline 32 & CABG AND random* & 2780 \\
\hline 33 & On-pump AND random* & 522 \\
\hline 34 & Cardiopulmonary bypass AND random* & 4474 \\
\hline 35 & Extracorporeal circulation AND random* & 4961 \\
\hline 36 & Conventional bypass AND random* & 916 \\
\hline
\end{tabular}

$O P C A B$, Off-pump coronary artery bypass; $O P C A B G$, off-pump coronary artery bypass grafting; $O N C A B$, on-pump coronary artery bypass. *Extended search query (randomized, randomised, randomly, random etc.). 


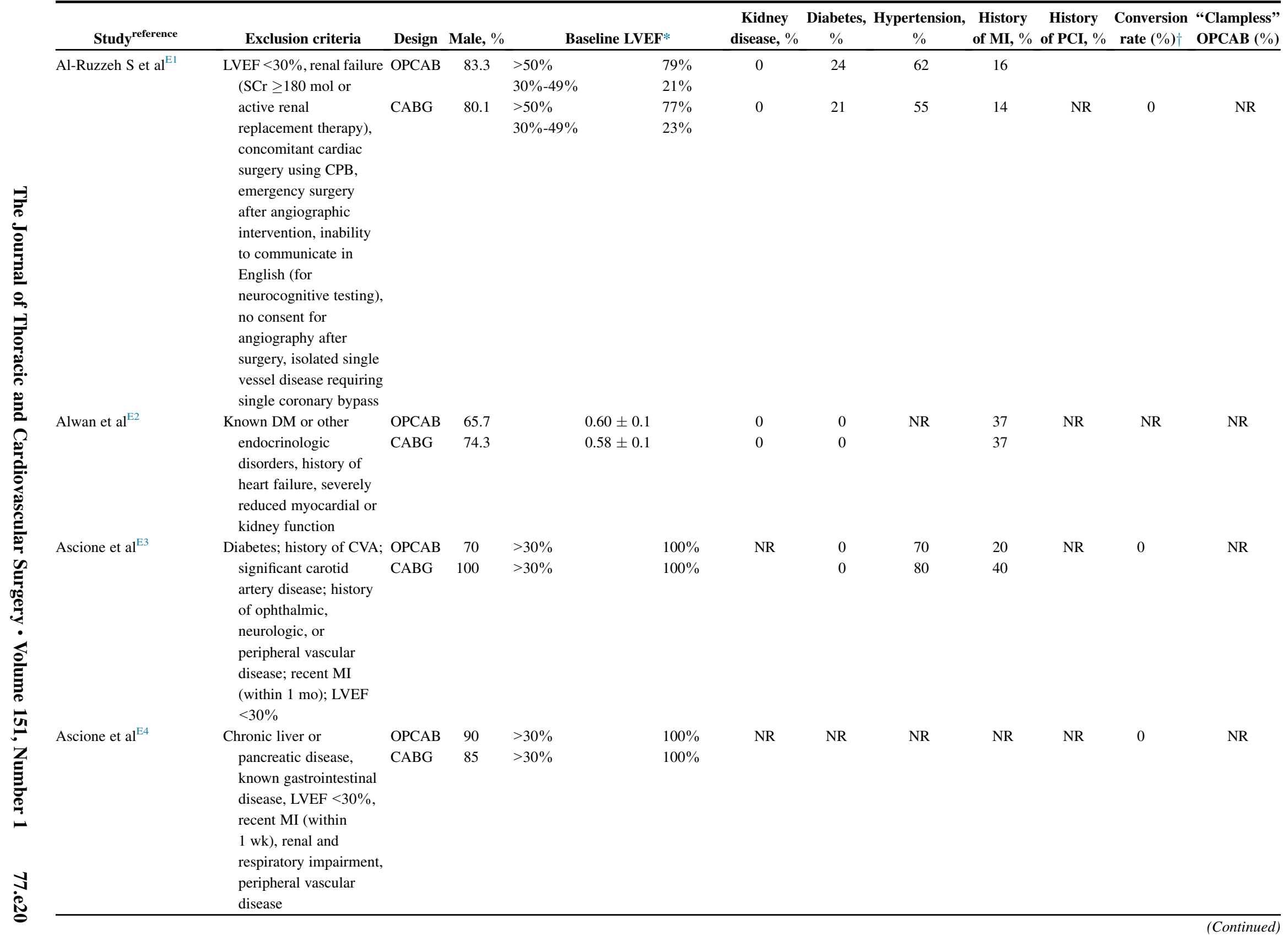




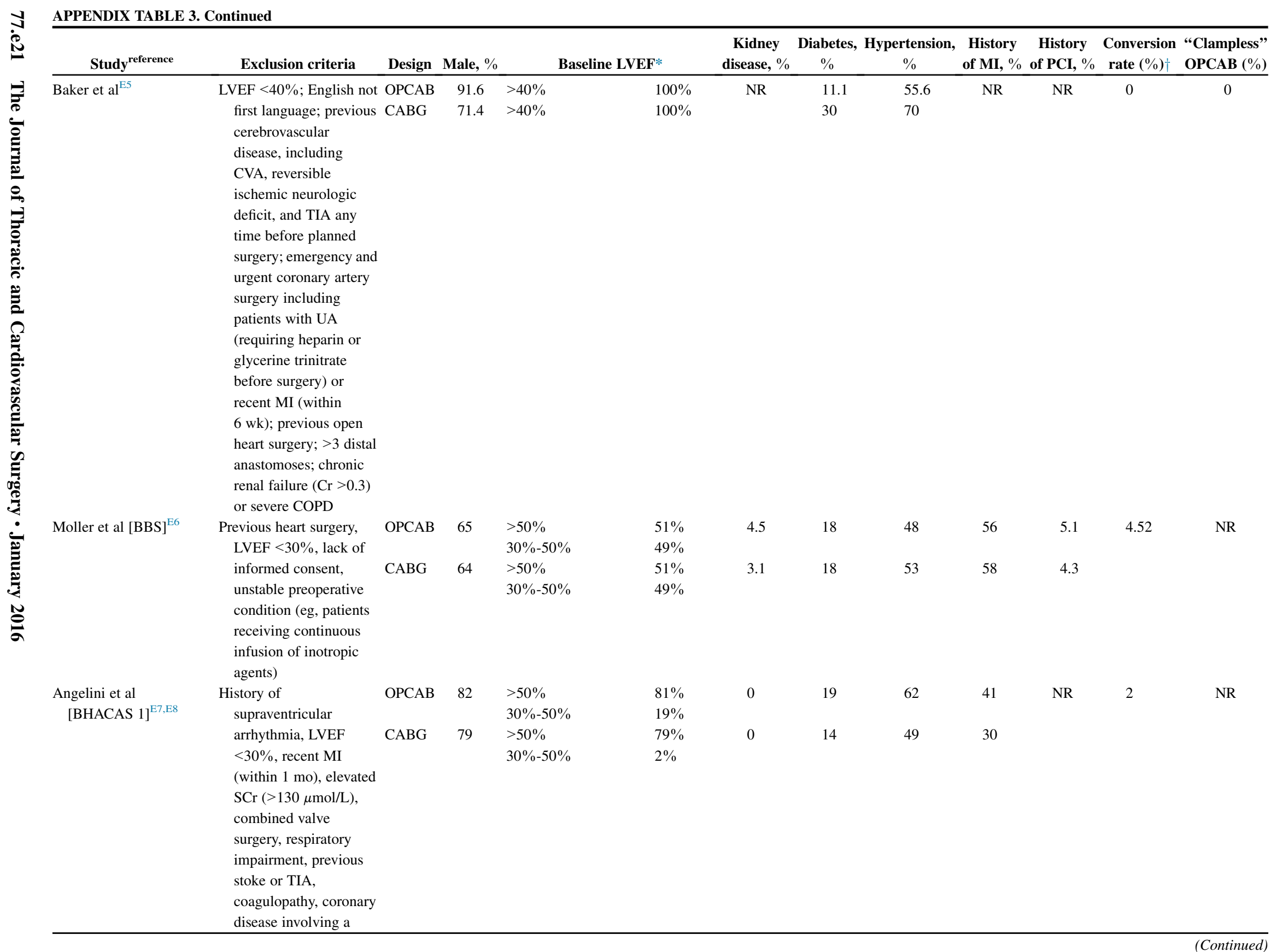




\begin{tabular}{|c|c|c|c|c|c|c|c|c|c|c|c|c|}
\hline Study ${ }^{\text {reference }}$ & Exclusion criteria & Design & Male, $\%$ & & Baseline LVEF* & $\begin{array}{c}\text { Kidney } \\
\text { disease, \% }\end{array}$ & $\begin{array}{c}\text { Diabetes, } \\
\% \\
\end{array}$ & $\begin{array}{c}\text { Hypertension, } \\
\% \\
\end{array}$ & $\begin{array}{c}\text { History } \\
\text { of MI, } \%\end{array}$ & $\begin{array}{c}\text { History } \\
\text { of PCI, \% }\end{array}$ & $\begin{array}{c}\text { Conversion } \\
\text { rate }(\%) \dagger\end{array}$ & $\begin{array}{l}\text { "Clampless" } \\
\text { OPCAB (\%) }\end{array}$ \\
\hline & $\begin{array}{l}\text { branches of the } \\
\text { circumflex artery distal } \\
\text { to the first obtuse } \\
\text { marginal or posterior } \\
\text { branches originating } \\
\text { from the left system }\end{array}$ & & & & & & & & & & & \\
\hline Angelini et al & History of & OPCAB & 85 & $<50 \%$ & $24 \%$ & 0 & 32 & 60 & 50 & NR & 0 & NR \\
\hline$\left[\right.$ BHACAS 2] ${ }^{\mathrm{E} 7}$ & $\begin{array}{l}\text { supraventricular } \\
\text { arrhythmia, LVEF } \\
<30 \%, \text { recent } \mathrm{MI} \\
\text { (within } 1 \mathrm{mo}), \text { elevated } \\
\mathrm{SCr}(>130 \mu \mathrm{mol} / \mathrm{L}), \\
\text { combined valve } \\
\text { surgery, respiratory } \\
\text { impairment, previous } \\
\text { stoke or TIA, } \\
\text { coagulapathy }\end{array}$ & CABG & 82 & $<50 \%$ & $23 \%$ & 0 & 30 & 50 & 50 & & & \\
\hline Bicer et $\mathrm{al}^{\mathrm{E} 9}$ & $\begin{array}{l}\text { Infection, redo surgery or } \\
\text { emergency surgery, } \\
\text { malignancies, } \\
\text { immunologic disorders, } \\
\text { recent MI (within last } \\
\text { mo); steroid use, } \\
\text { NYHA class IV heart } \\
\text { failure, cardiogenic } \\
\text { shock, severe valvular } \\
\text { disease, renal failure, } \\
\text { and hepatic failure }\end{array}$ & $\begin{array}{l}\text { OPCAB } \\
\text { CABG }\end{array}$ & $\begin{array}{l}88 \\
88\end{array}$ & & $\begin{array}{c}53.76 \pm 15.07 \% \\
52.31 \pm 10.8 \%\end{array}$ & NR & $\begin{array}{l}24 \\
12\end{array}$ & $\begin{array}{l}68 \\
68\end{array}$ & NR & NR & 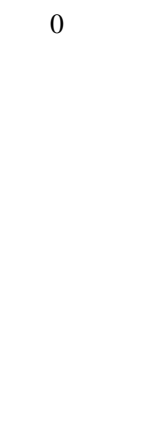 & 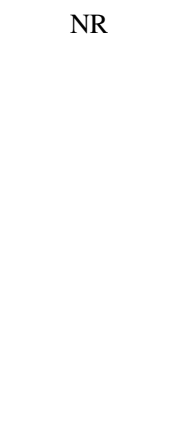 \\
\hline Blacher et $\mathrm{al}^{\mathrm{E} 10}$ & Previous heart surgery; & OPCAB & 77 & $>40 \%$ & $100 \%$ & 0 & 8 & 77 & 62 & NR & 0 & NR \\
\hline & $\begin{array}{l}\text { acute recent MI (within } \\
3 \text { mo); need for } \\
\text { concomitant cardiac } \\
\text { procedure (valve } \\
\text { replacement, correction } \\
\text { of congenital defects, or } \\
\text { other surgical } \\
\text { procedures); renal } \\
\text { failure, defined as SCr } \\
>177 \mu \mathrm{mol} / \mathrm{L} ; \\
\text { immunodeficiency } \\
\text { syndrome; chronic or } \\
\end{array}$ & CABG & 53 & $>40 \%$ & $100 \%$ & 0 & 13 & 68 & 48 & & & \\
\hline
\end{tabular}




\begin{tabular}{|c|c|c|c|c|c|c|c|c|c|c|c|c|}
\hline Study ${ }^{\text {reference }}$ & Exclusion criteria & Design & Male, $\%$ & & Baseline LVEF* & $\begin{array}{c}\text { Kidney } \\
\text { disease, \% }\end{array}$ & $\begin{array}{c}\text { Diabetes, } \\
\%\end{array}$ & $\begin{array}{c}\text { Hypertension, } \\
\% \\
\end{array}$ & $\begin{array}{c}\text { History } \\
\text { of MI, \% }\end{array}$ & $\begin{array}{c}\text { History } \\
\text { of PCI, \% }\end{array}$ & $\begin{array}{c}\text { Conversion } \\
\text { rate }(\%) \dagger\end{array}$ & $\begin{array}{l}\text { "Clampless" } \\
\text { OPCAB }(\%)\end{array}$ \\
\hline & $\begin{array}{l}\text { perioperative } \\
\text { corticotherapy; LVEF } \\
<40 \% \text {; active } \\
\text { infection; UA requiring } \\
\text { intensive care; NYHA } \\
\text { class III or IV }\end{array}$ & & & & & & & & & & & \\
\hline \multirow[t]{2}{*}{ Bonacchi et al $^{\mathrm{E} 11}$} & LVEF $<35 \%$, age $70 \mathrm{y}$ & OPCAB & 66.6 & & $55 \pm 8.6 \%$ & 0 & NR & 77.8 & 22.2 & 33.3 & 5.55 & NR \\
\hline & $\begin{array}{l}\text { previous MI, redo } \\
\text { surgery, presence of } \\
\text { valvular heart disease } \\
\text { and/or cerebrovascular } \\
\text { disease, abnormal } \\
\text { preoperative carotid } \\
\text { vessel angiography, } \\
\text { coronary artery disease } \\
\text { involving the distal } \\
\text { circumflex artery, renal } \\
\text { dysfunction, } \\
\text { coagulopathy }\end{array}$ & CABG & 71 & & $53 \pm 7.4 \%$ & 0 & & 54.2 & 21 & 29.2 & & \\
\hline \multirow[t]{2}{*}{ Caputo et $\mathrm{al}^{\mathrm{E} 12}$} & $\mathrm{LVEF}<30 \%$, recent MI & OPCAB & 85 & & NR & 0 & 0 & NR & 30 & NR & 0 & NR \\
\hline & $\begin{array}{l}\text { (within last mo), type II } \\
\text { DM, respiratory or } \\
\text { renal impairment, } \\
\text { coagulopathy, previous } \\
\text { CVA }\end{array}$ & CABG & 95 & & & 0 & 0 & & 35 & & & \\
\hline \multirow[t]{2}{*}{ Carrier et al ${ }^{\mathrm{E} 13}$} & Need for CPB for valve & OPCAB & 67.9 & $<45 \%$ & $100 \%$ & 7 & 50 & 82 & NR & NR & 15.63 & NR \\
\hline & $\begin{array}{l}\text { surgery or another } \\
\text { reason }\end{array}$ & CABG & 83.8 & $<45 \%$ & $100 \%$ & 11 & 46 & 81 & & & & \\
\hline \multirow[t]{2}{*}{ Cavalca et $\mathrm{al}^{\mathrm{E} 14}$} & Porcelain aorta, Q-wave & OPCAB & 82 & & $55.8 \pm 9.6 \%$ & 0 & 23 & 55 & 36 & NR & 4.54 & NR \\
\hline & $\begin{array}{l}\text { MI in the previous } \\
6 \mathrm{wk}, \mathrm{UA}, \mathrm{LVEF}<30 \%\end{array}$ & CABG & 68 & & $56.2 \pm 13.4 \%$ & 14 & 28 & 64 & 48 & & & \\
\hline \multirow[t]{2}{*}{ Chowdhury et $\mathrm{al}^{\mathrm{E} 15}$} & Emergency surgery, AMI & OPCAB & 80 & & $0.54 \pm 0.64$ & 0 & 32 & 60 & 44 & NR & 0 & NR \\
\hline & $\begin{array}{l}\text { or recent MI (within } \\
2 \text { wk), treatment with } \\
\text { fibrinolytic agents } \\
\text { within } 48 \mathrm{~h}, \text { redo } \\
\text { surgery, LVEF }<30 \%, \\
\text { concomitant valvular } \\
\text { heart disease, UA, renal } \\
\text { disease, COPD, steroid } \\
\text { therapy, hepatic disease }\end{array}$ & CABG & 84 & & $0.52 \pm 0.60 \%$ & 0 & 28 & 56 & 40 & & & \\
\hline
\end{tabular}




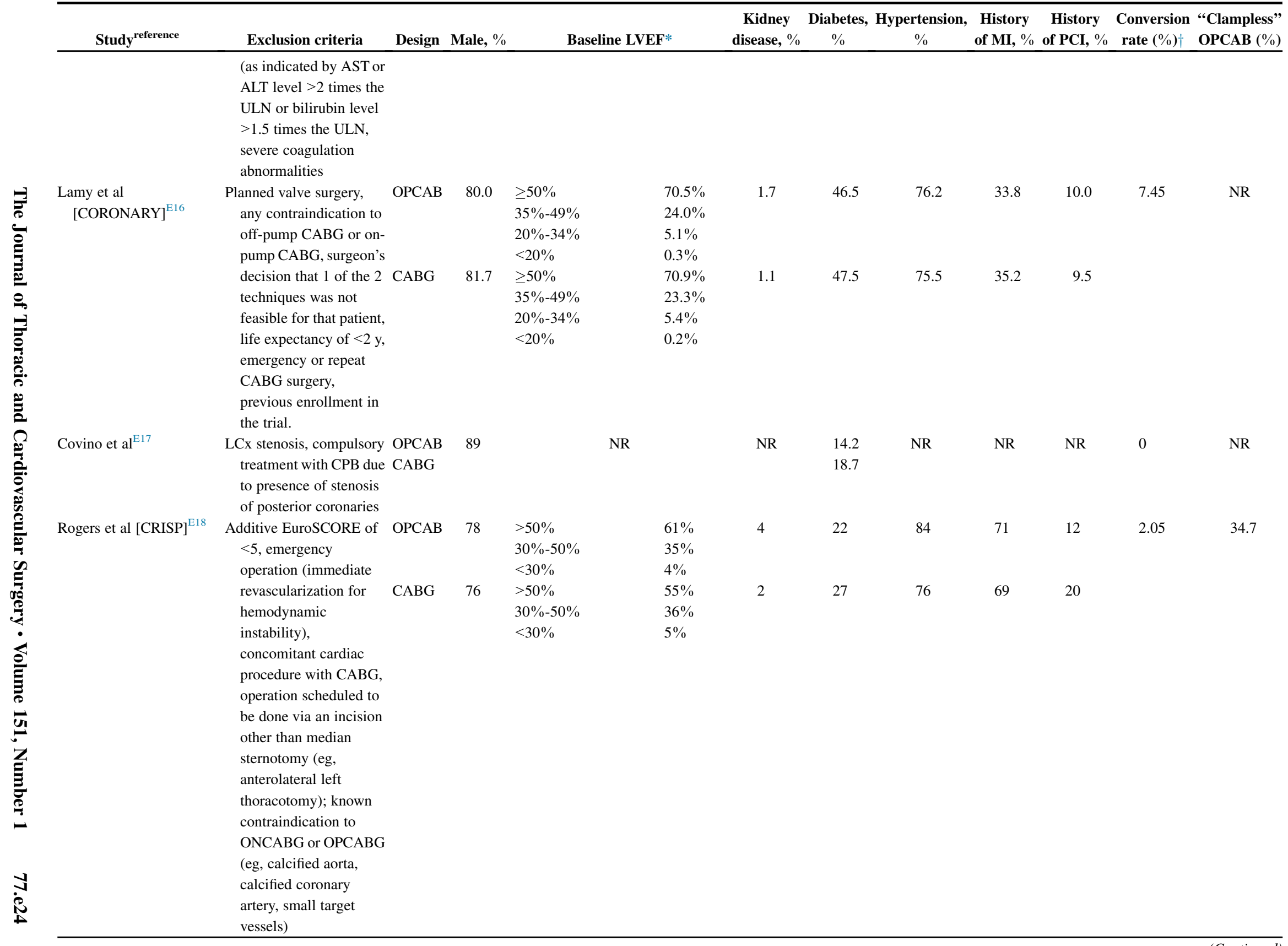




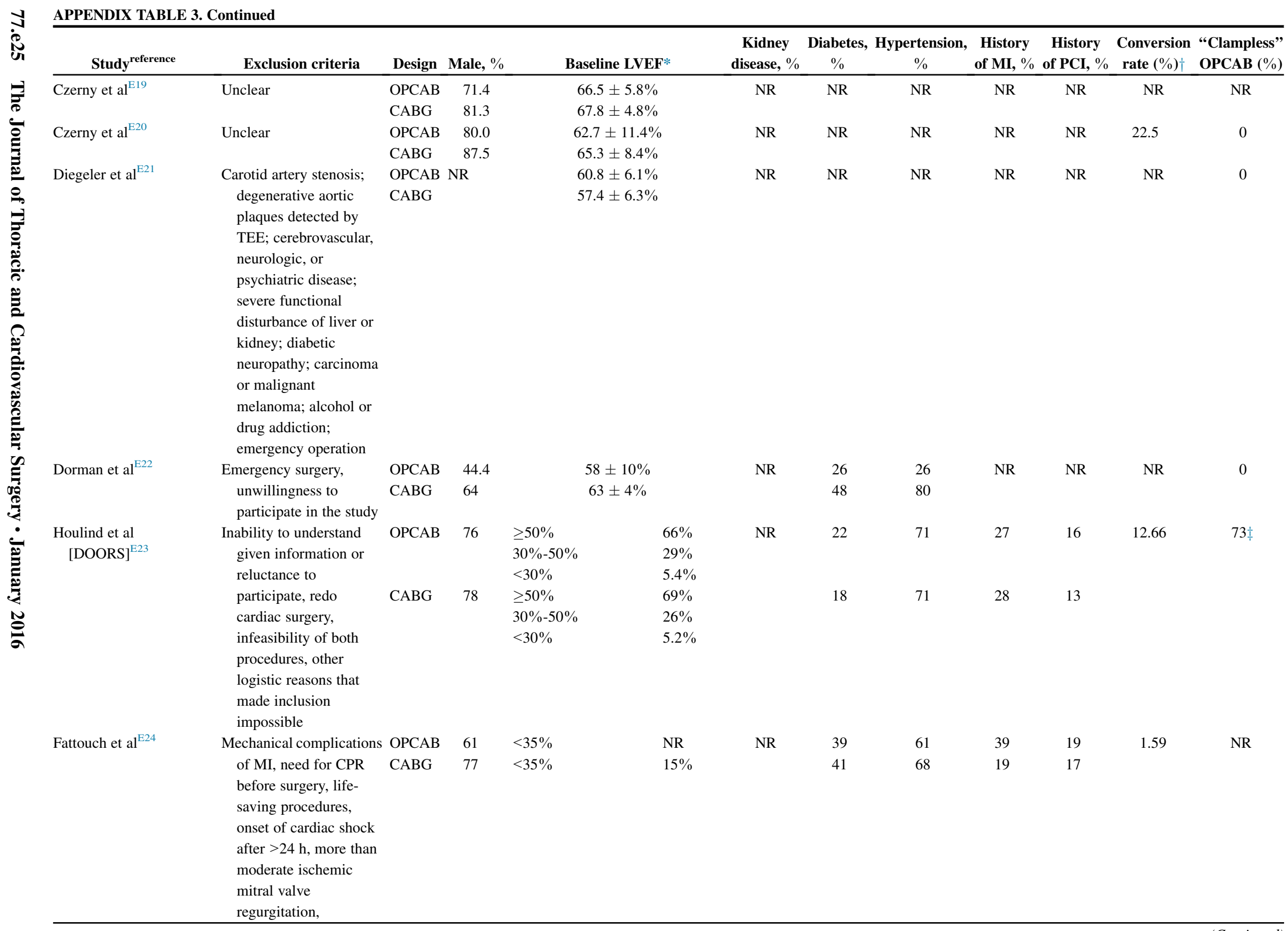




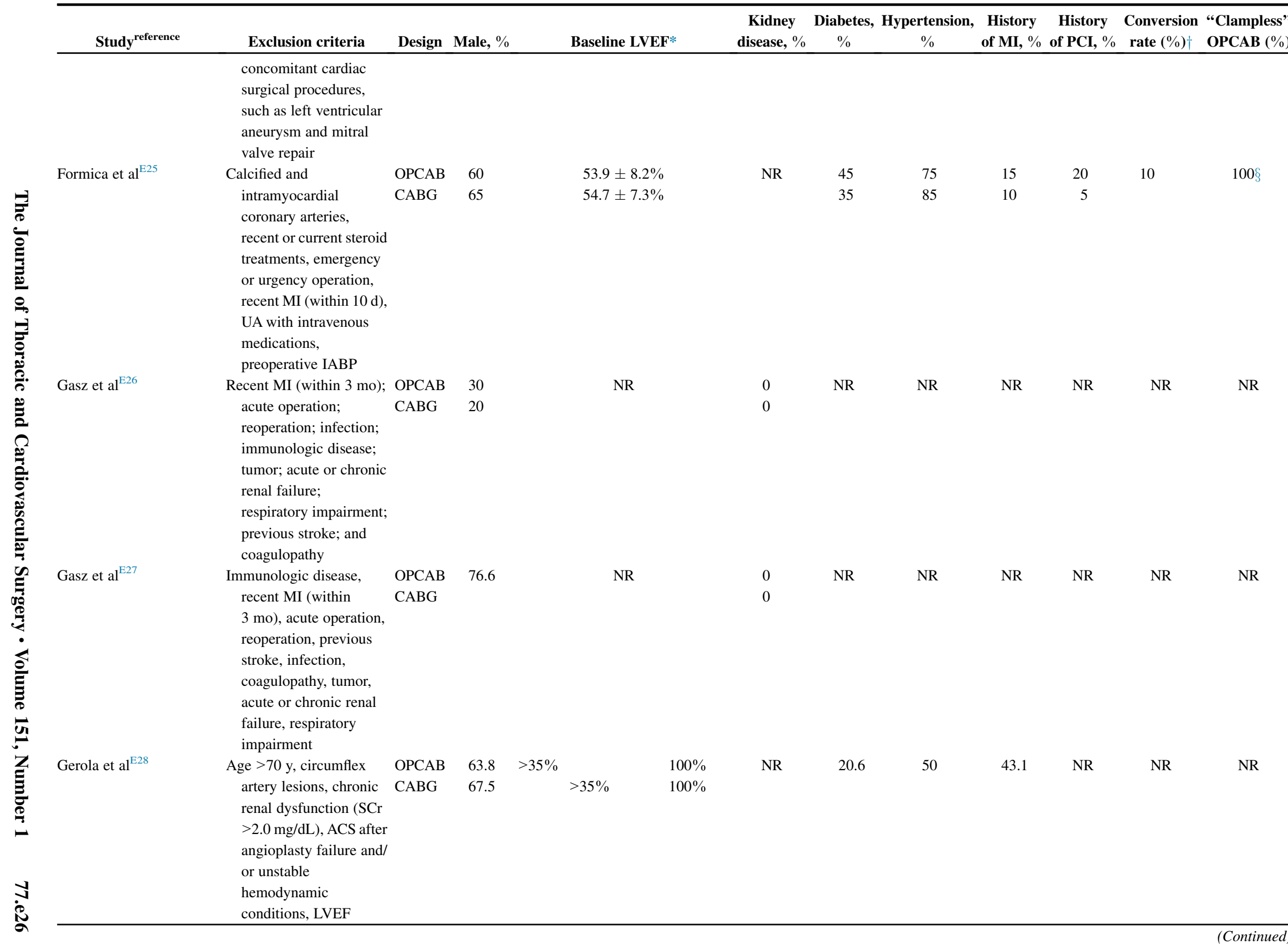




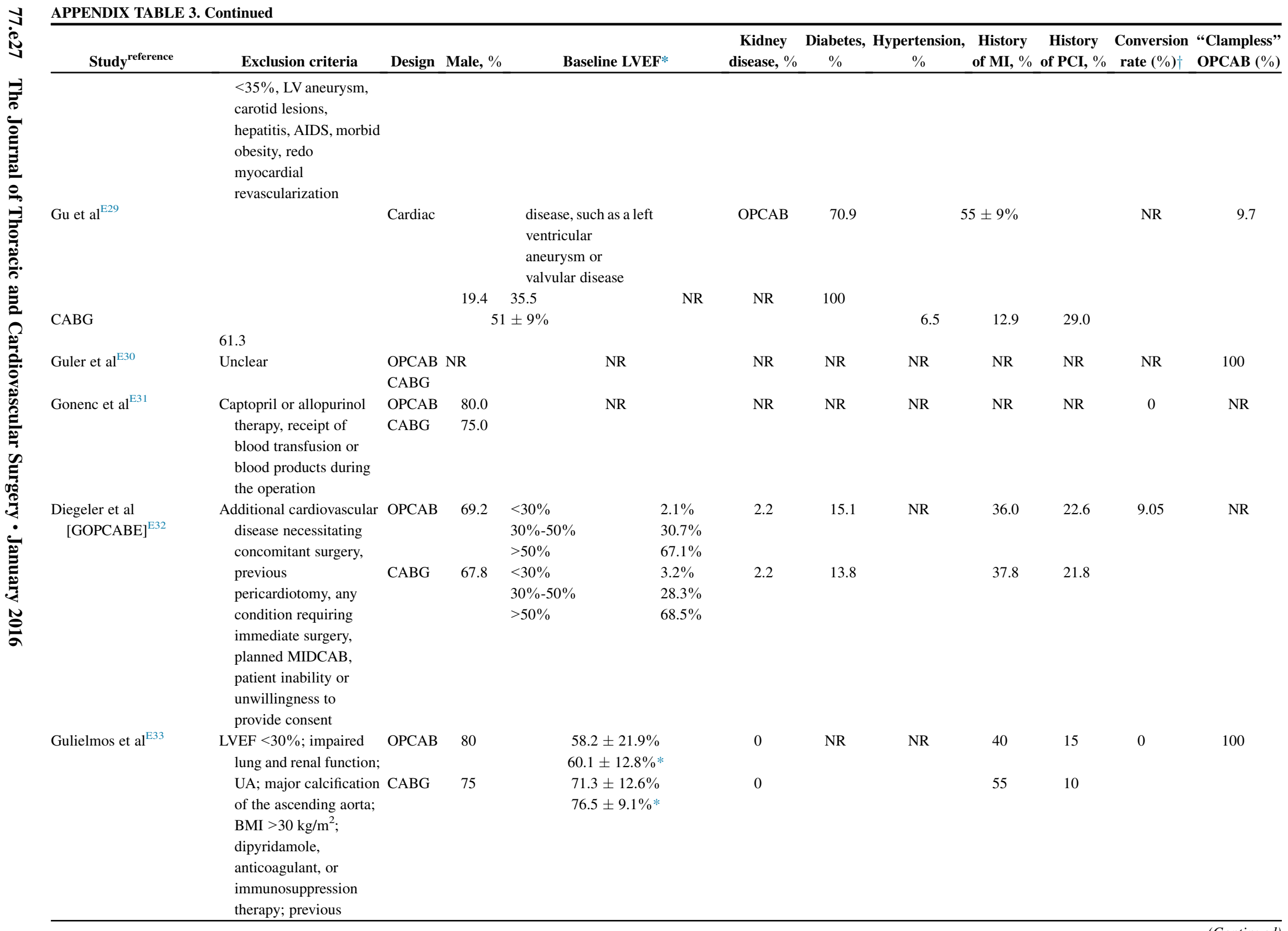




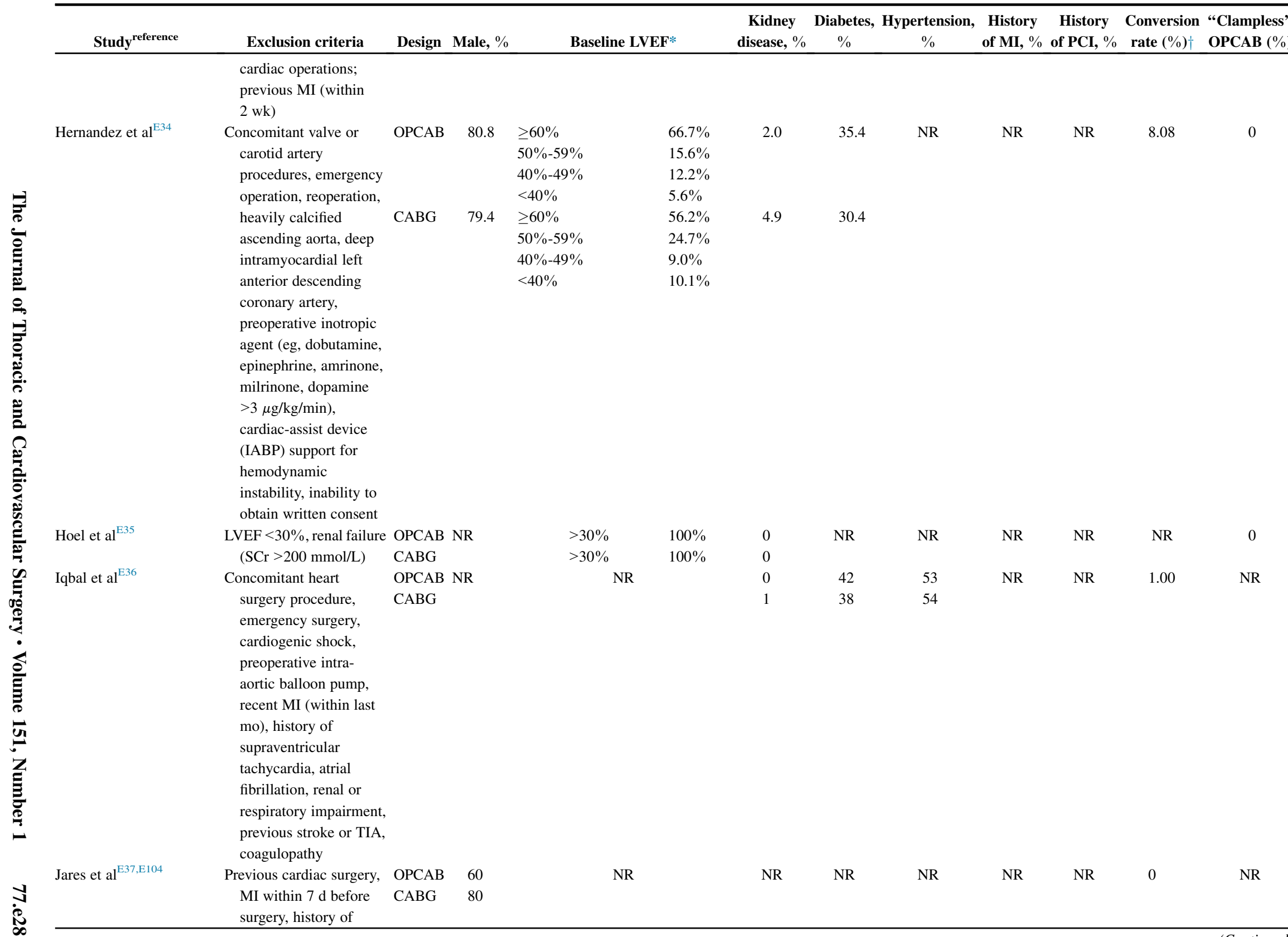




\begin{tabular}{|c|c|c|c|c|c|c|c|c|c|c|c|}
\hline Study ${ }^{\text {reference }}$ & Exclusion criteria & Design & Male, $\%$ & Baseline LVEF* & $\begin{array}{c}\text { Kidney } \\
\text { disease, \% }\end{array}$ & $\begin{array}{c}\text { Diabetes, } \\
\%\end{array}$ & $\begin{array}{c}\text { Hypertension, } \\
\%\end{array}$ & $\begin{array}{c}\text { History } \\
\text { of MI, \% }\end{array}$ & $\begin{array}{c}\text { History } \\
\text { of PCI, } \%\end{array}$ & $\begin{array}{c}\text { Conversion } \\
\text { rate }(\%) \dagger\end{array}$ & $\begin{array}{l}\text { "Clampless" } \\
\text { OPCAB (\%) }\end{array}$ \\
\hline & $\begin{array}{l}\text { hematologic or liver } \\
\text { disorders, renal } \\
\text { insufficiency (sCR } \\
>150 \mu \text { mol/L), } \\
\text { preoperative anemia } \\
\text { (hemoglobin, }<11 \mathrm{~g} / \\
\text { dL; hematocrit, }<32 \text { ) }\end{array}$ & & & & & & & & & & \\
\hline $\begin{array}{l}\text { Johansson-Synnergren } \\
\text { et al }{ }^{\mathrm{E} 39}\end{array}$ & $\begin{array}{l}\text { Stenoses in the distal } \\
\text { circumflex area, LVEF } \\
<30 \% \text {, preoperative } \\
\text { stroke, known } \\
\text { peripheral vascular } \\
\text { disease, infection or } \\
\text { preoperative treatment } \\
\text { with anti-inflammatory } \\
\text { drugs }\end{array}$ & $\begin{array}{l}\text { OPCAB } \\
\text { CABG }\end{array}$ & $\begin{array}{l}69.2 \\
61.5\end{array}$ & $\begin{array}{l}63.6 \pm 2.6 \% \\
60.6 \pm 2.7 \%\end{array}$ & NR & NR & NR & NR & NR & 0 & NR \\
\hline
\end{tabular}




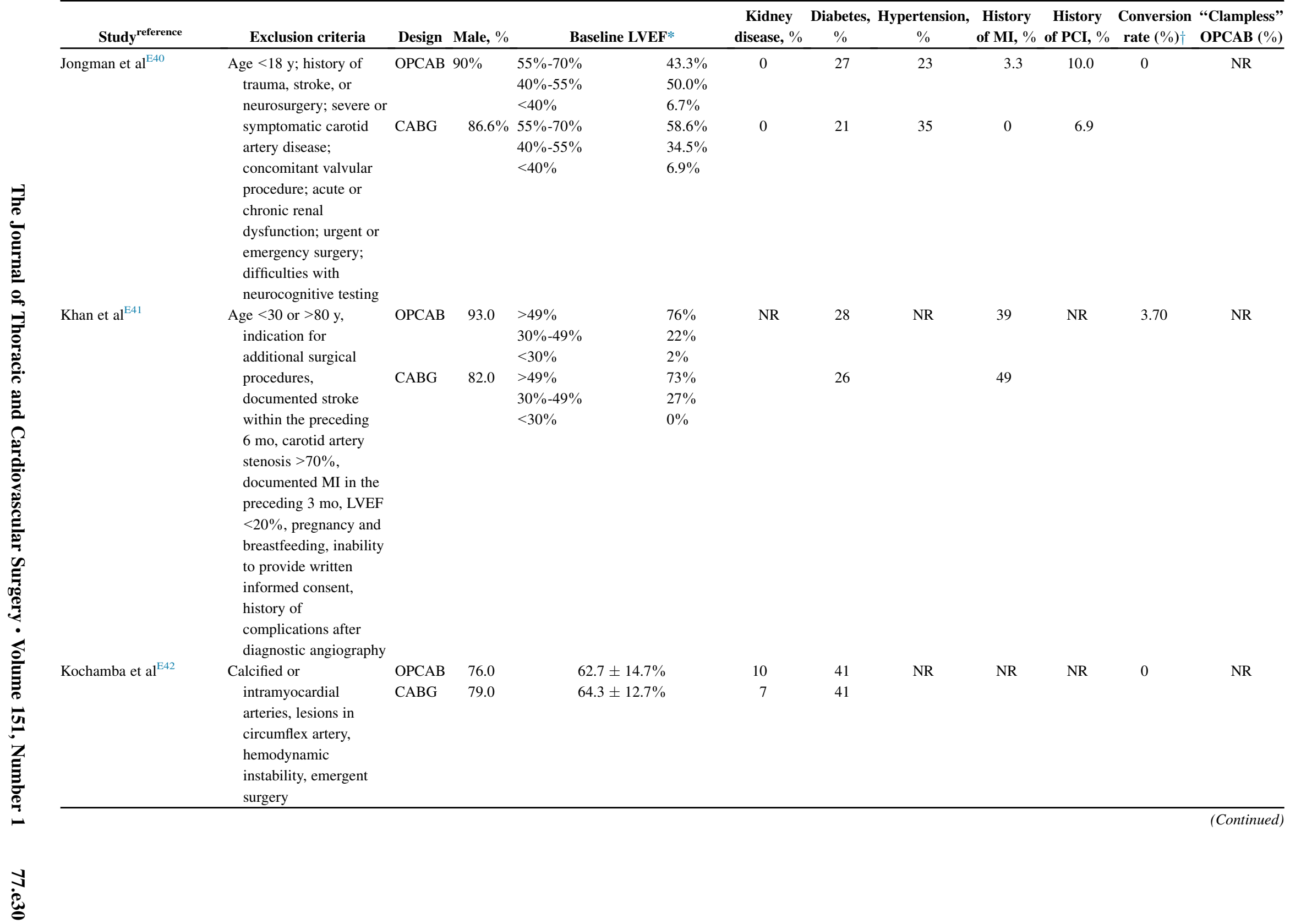




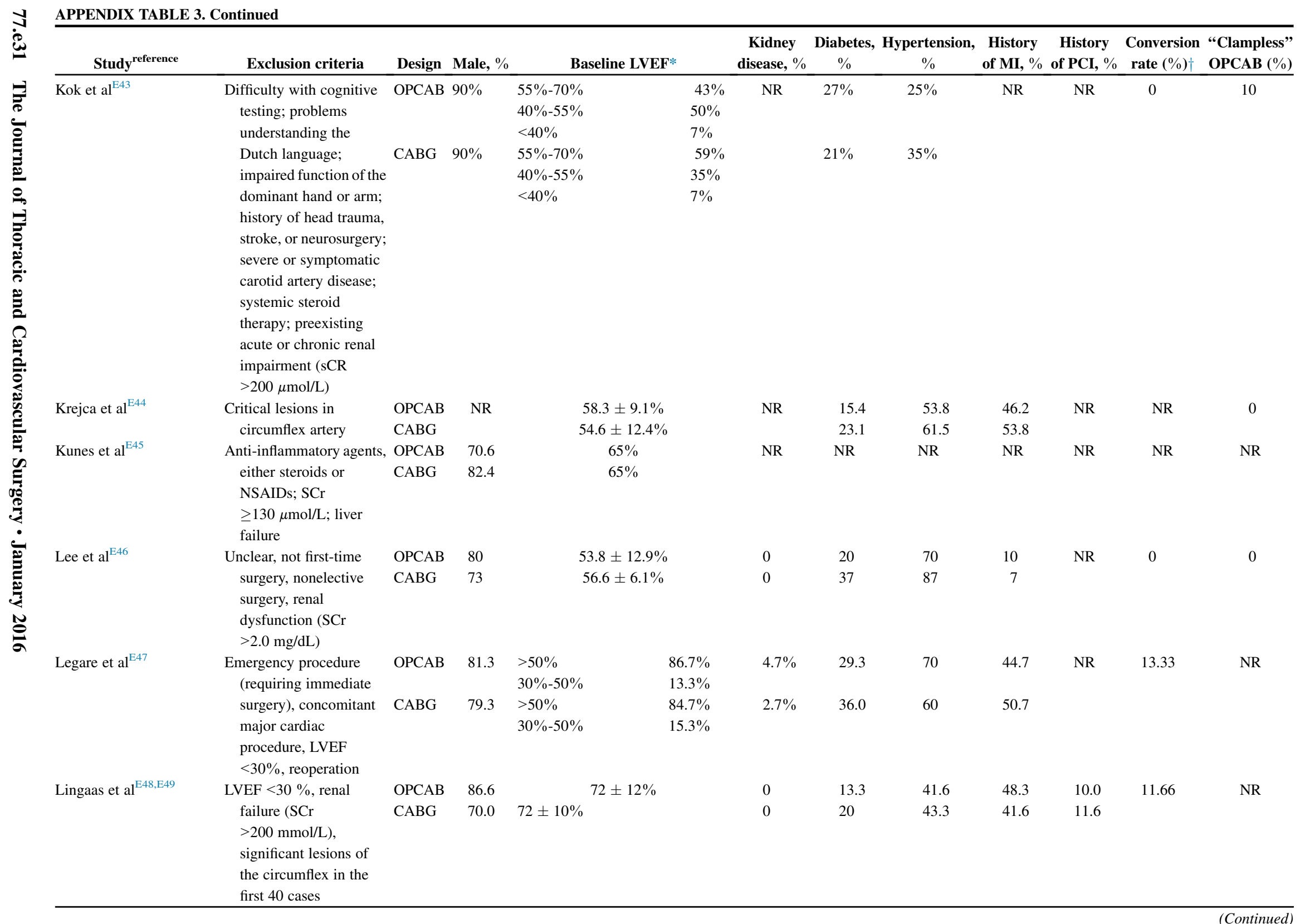




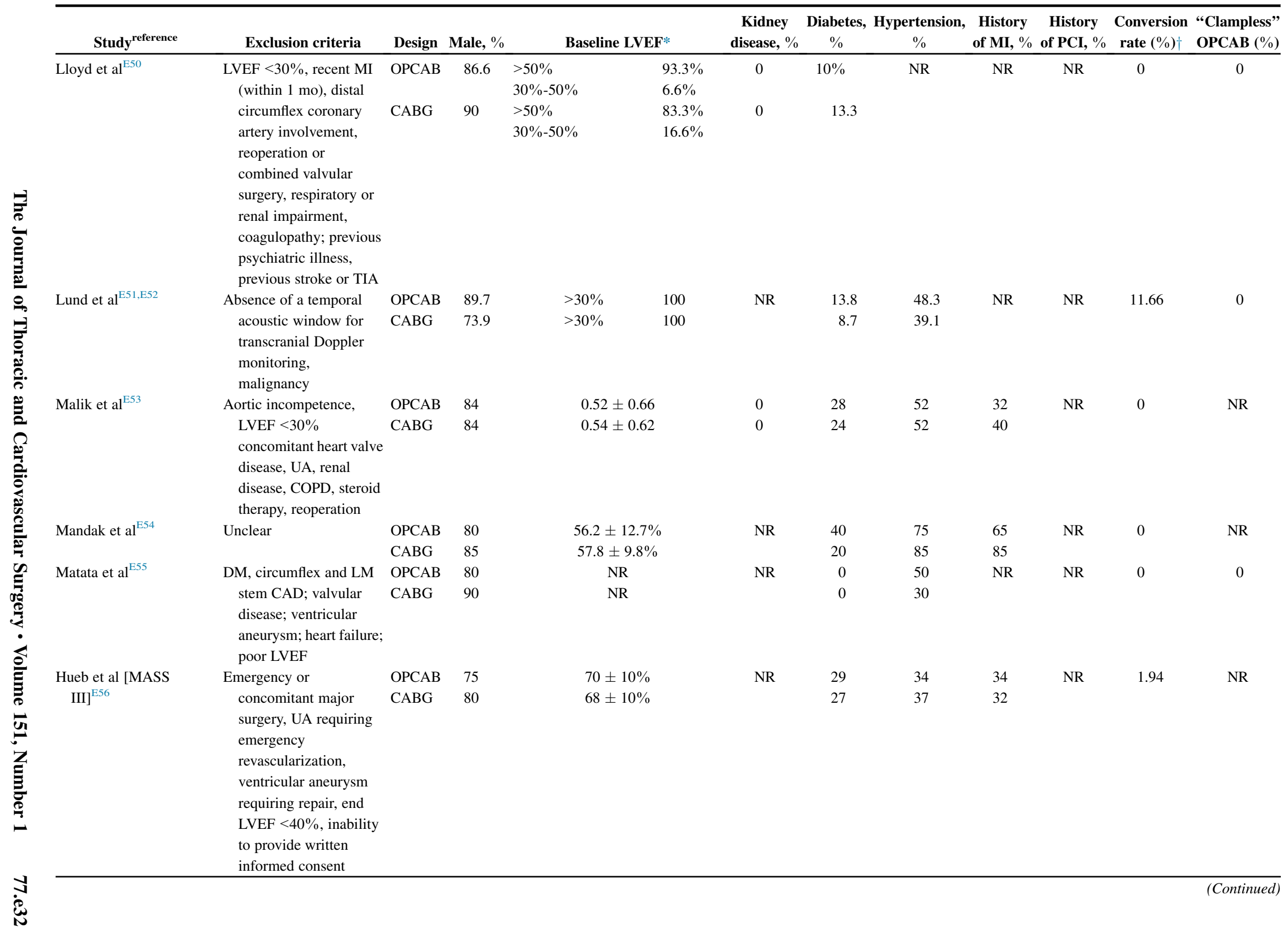




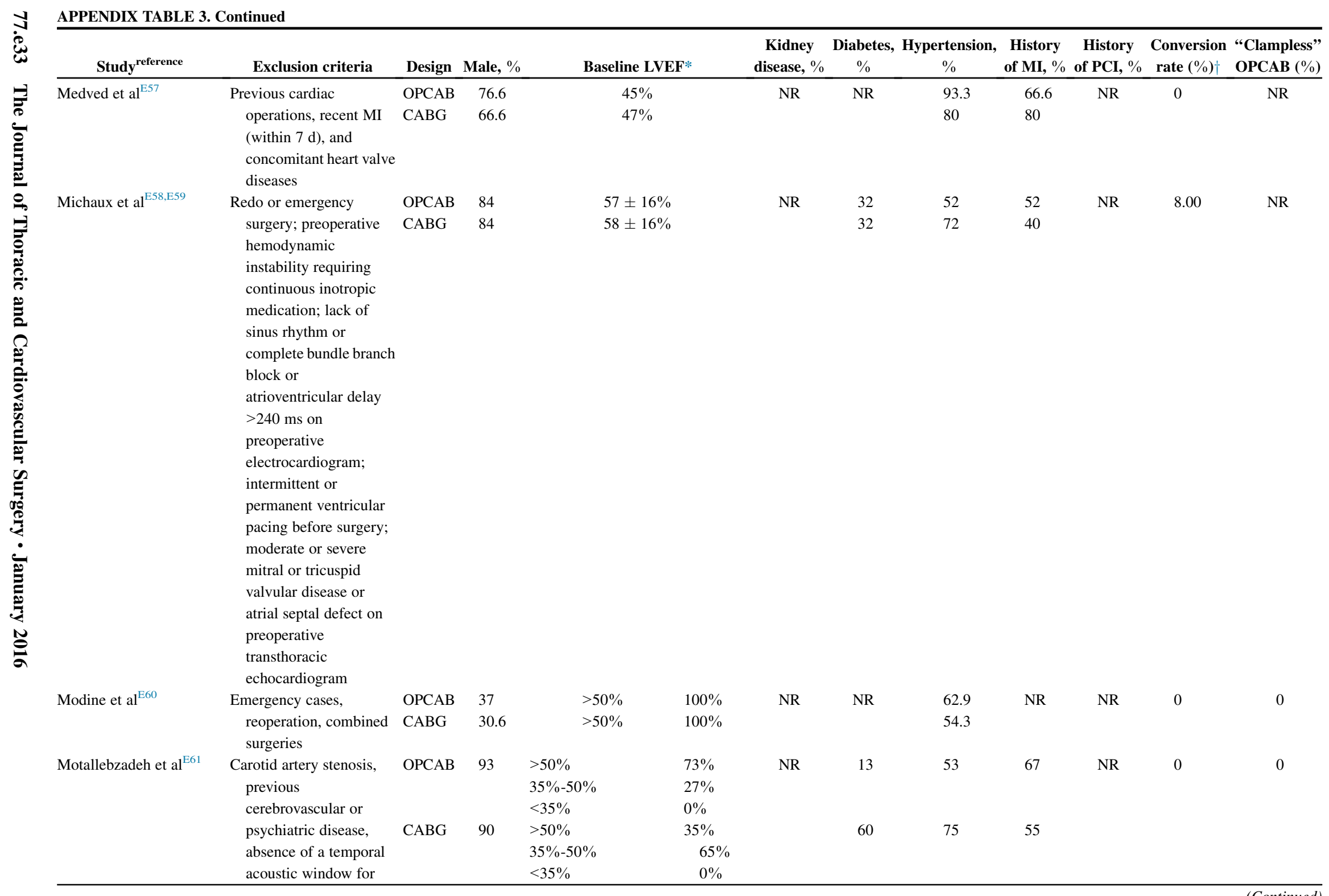




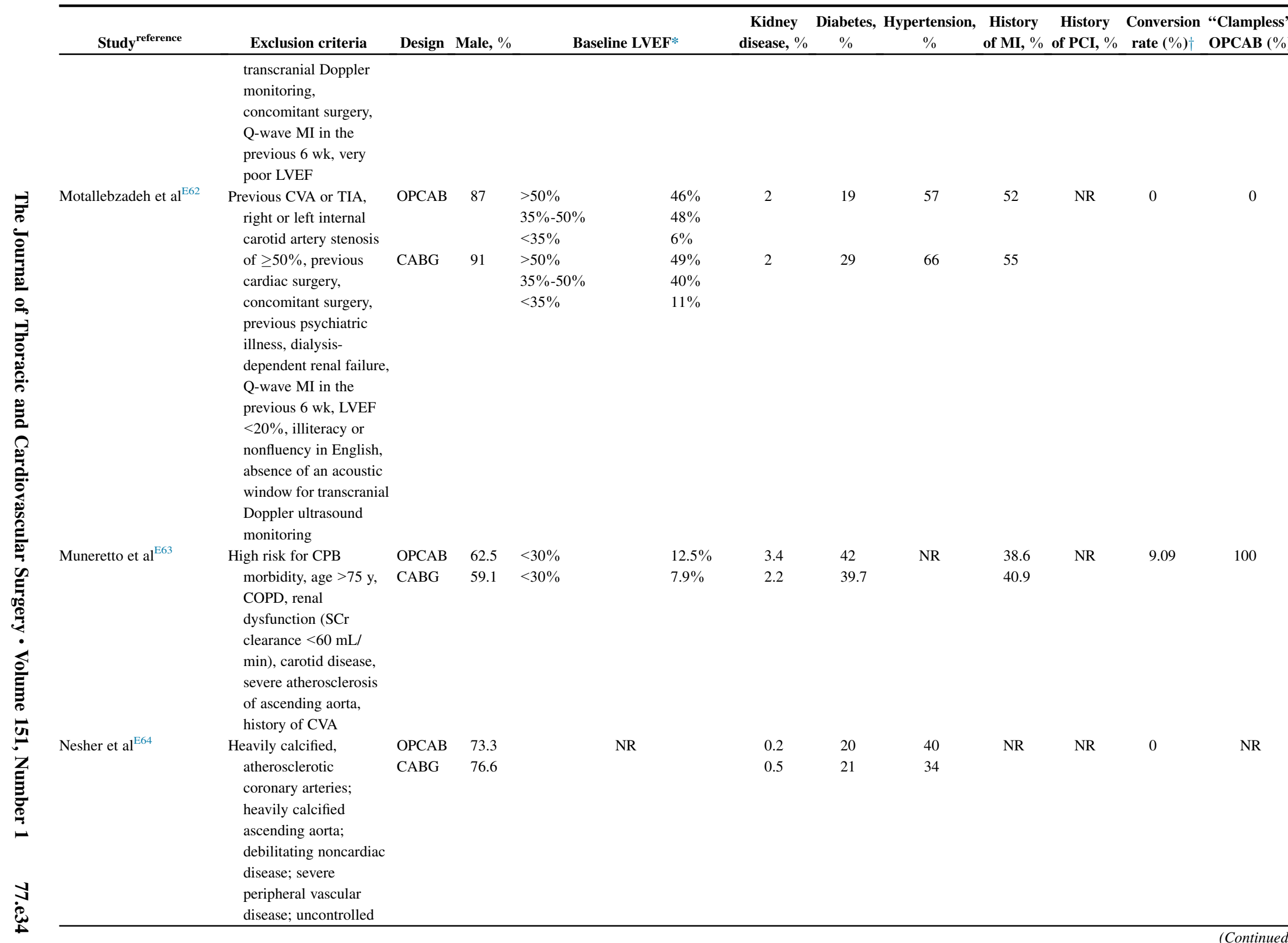




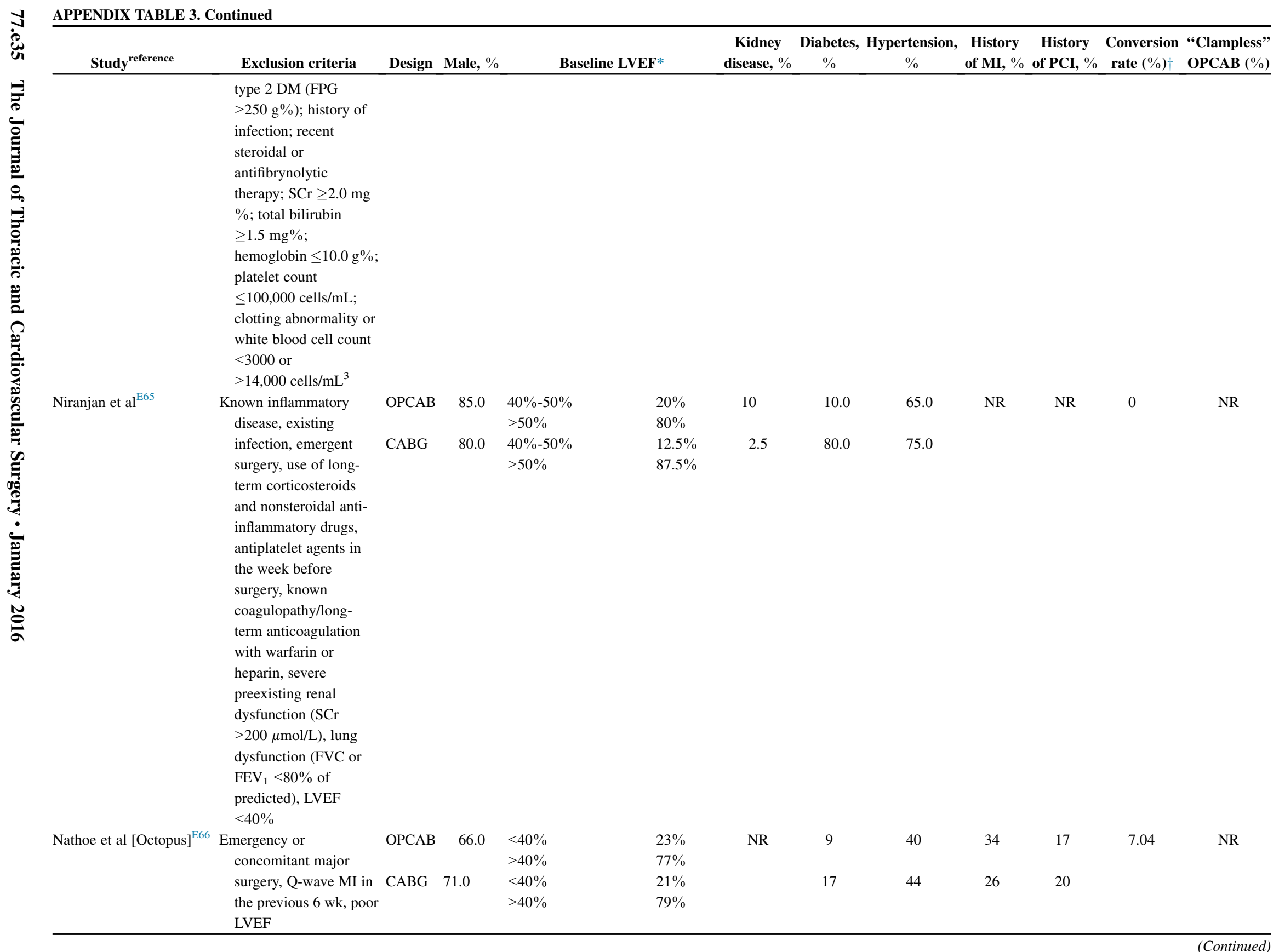




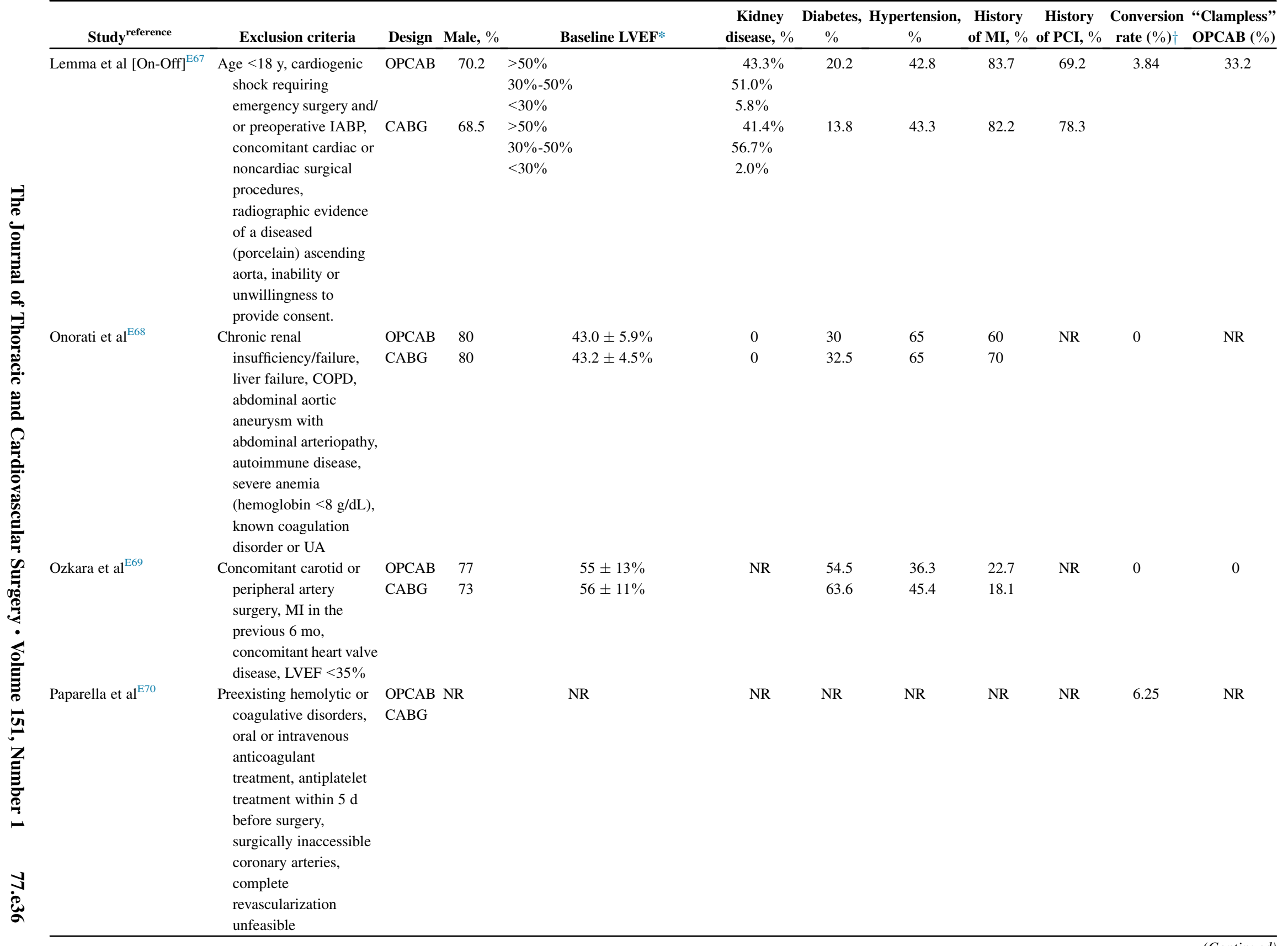




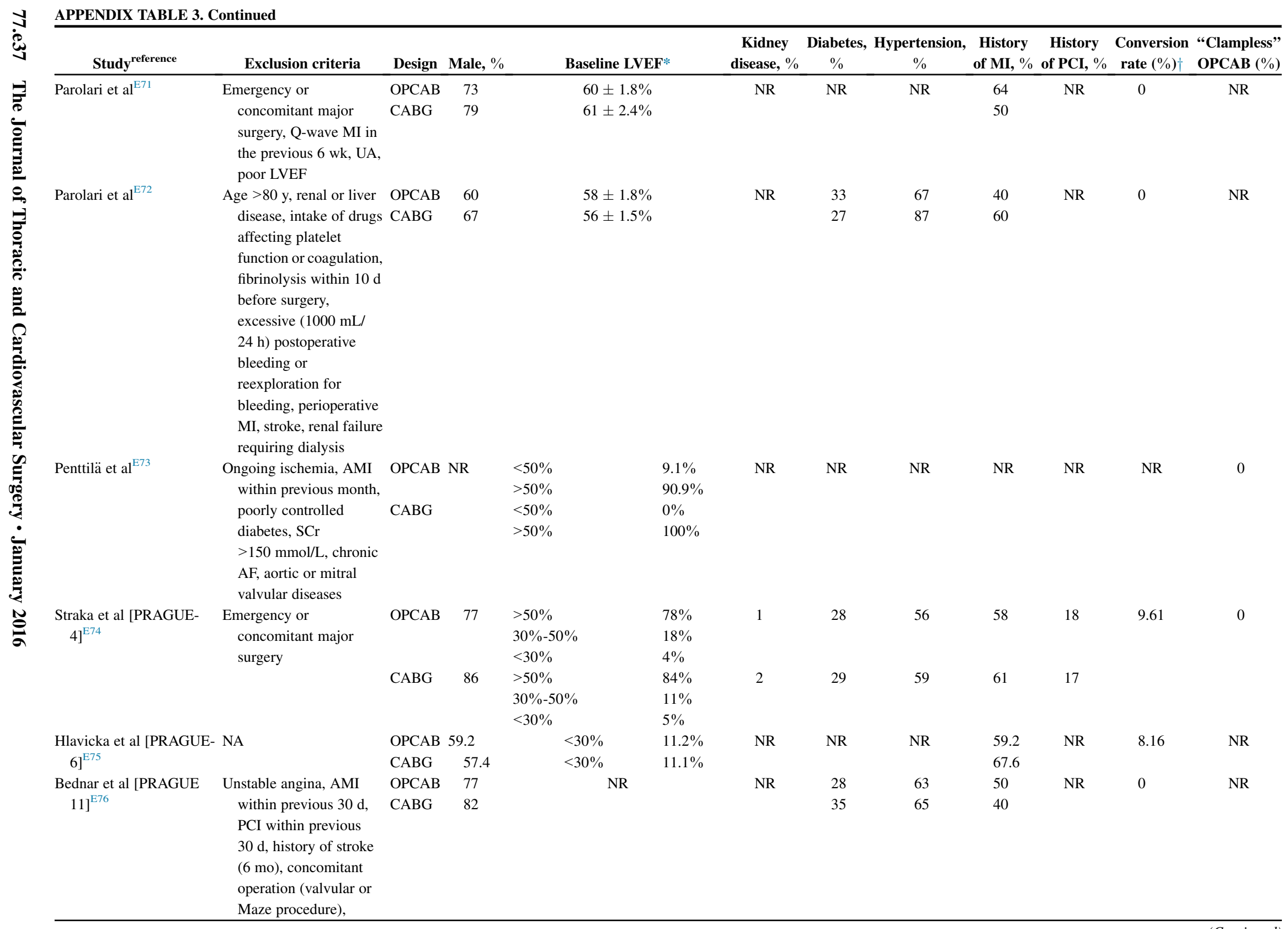




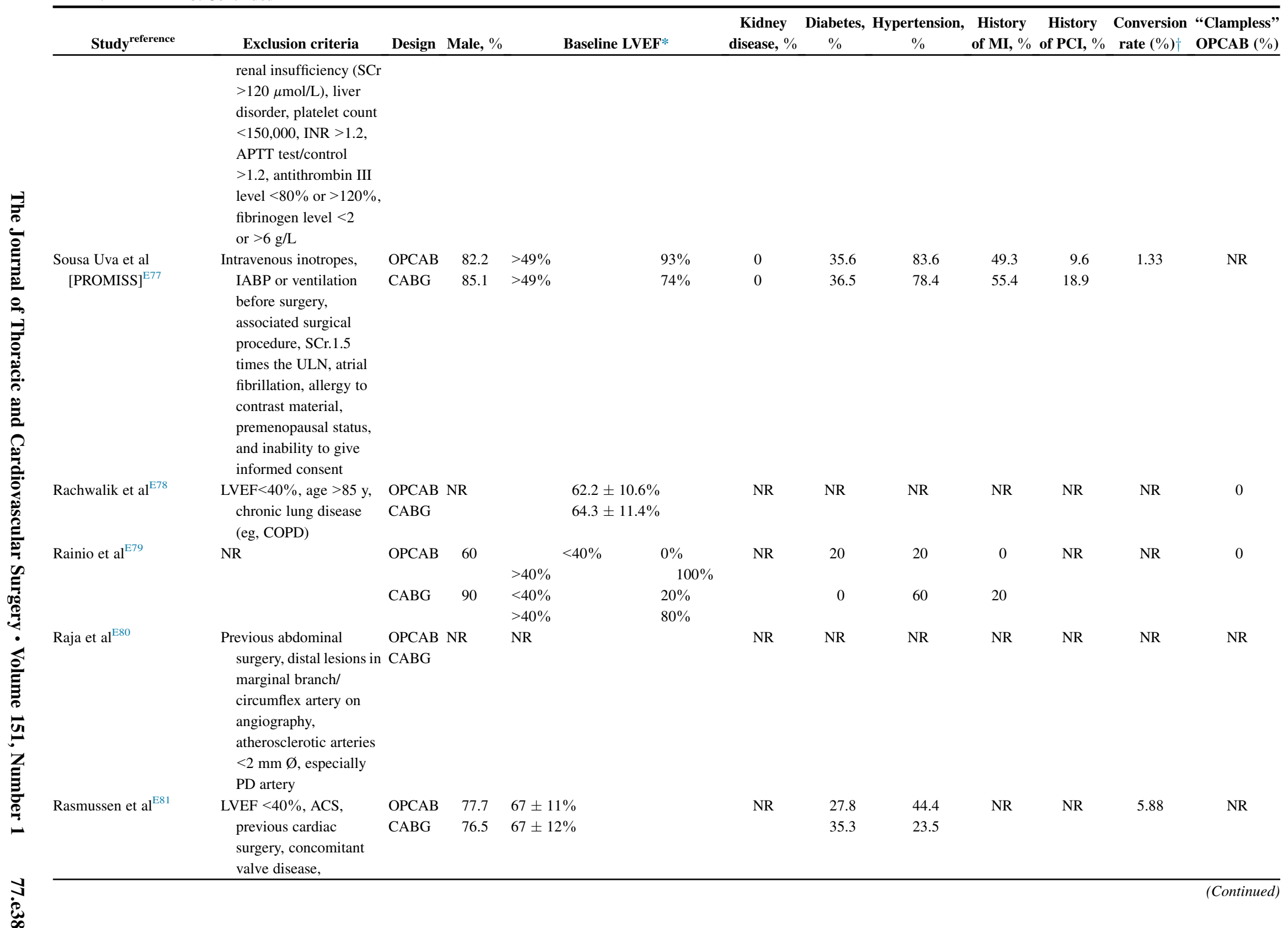




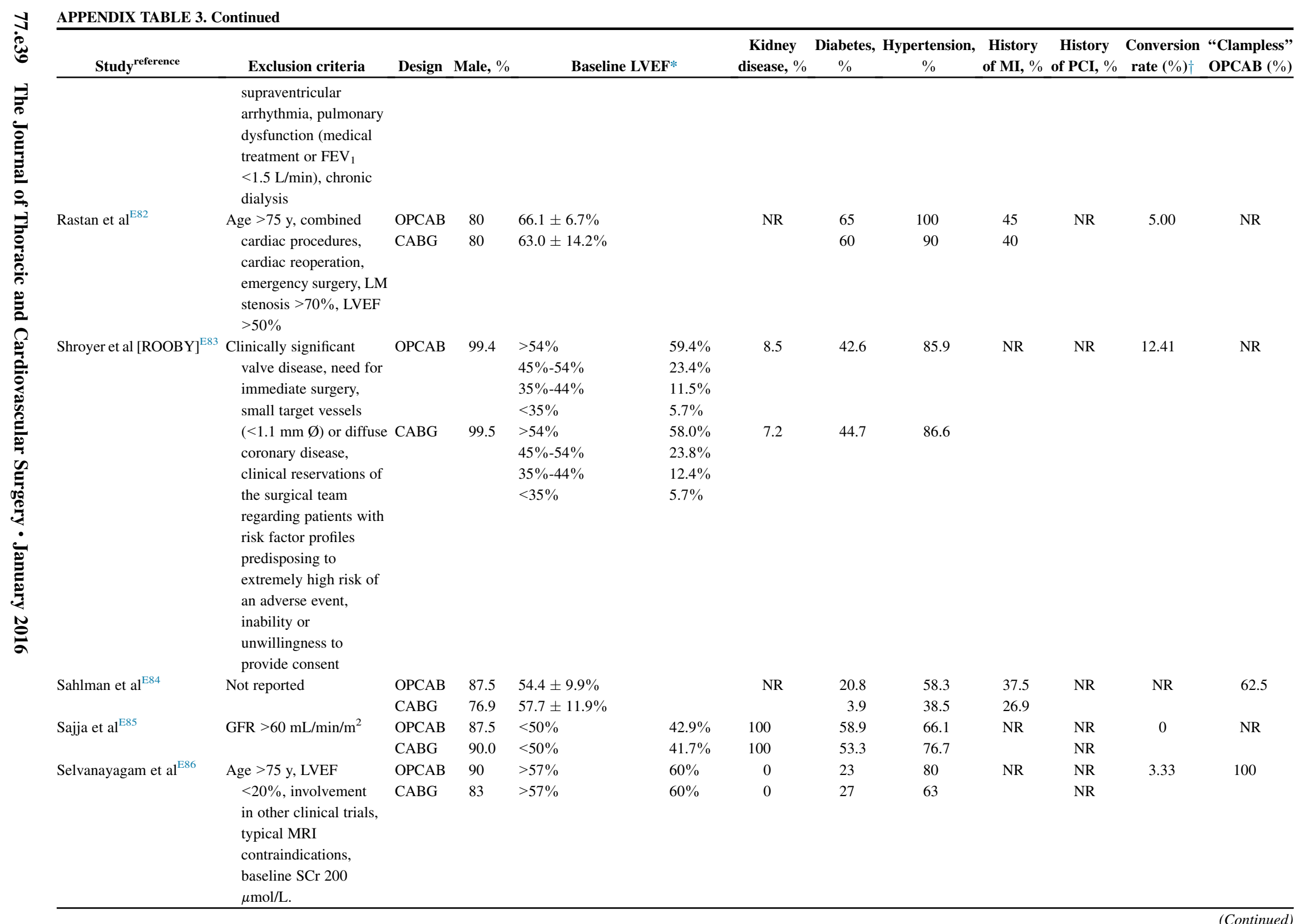




\begin{tabular}{|c|c|c|c|c|c|c|c|c|c|c|c|c|}
\hline Study ${ }^{\text {reference }}$ & Exclusion criteria & Design & Male, $\%$ & Baseline I & & $\begin{array}{c}\text { Kidney } \\
\text { disease, \% }\end{array}$ & $\begin{array}{c}\text { Diabetes, } \\
\% \\
\end{array}$ & $\begin{array}{c}\text { Hypertension, } \\
\% \\
\end{array}$ & $\begin{array}{c}\text { History } \\
\text { of MI, \% }\end{array}$ & $\begin{array}{c}\text { History } \\
\text { of PCI, \% }\end{array}$ & $\begin{array}{c}\text { Conversion } \\
\text { rate }(\%) \dagger \\
\end{array}$ & $\begin{array}{l}\text { "Clampless" } \\
\text { OPCAB (\%) }\end{array}$ \\
\hline \multirow[t]{10}{*}{ Puskas et al [SMART] ${ }^{\mathrm{E} 87}$} & Cardiogenic shock and & OPCAB & 78 & $>54 \%$ & $52 \%$ & 4 & 33 & 65 & 35 & 9 & 1.00 & 41 \\
\hline & IABP; chronic renal & & & $45 \%-54 \%$ & $22 \%$ & & & & & & & \\
\hline & insufficiency with SCr & & & $35 \%-44 \%$ & $16 \%$ & & & & & & & \\
\hline & $>2.5 \mathrm{mg} / \mathrm{dL}$ were & & & $25 \%-34 \%$ & $6 \%$ & & & & & & & \\
\hline & exempt from & & & $<25 \%$ & $5 \%$ & & & & & & & \\
\hline & postoperative cardiac & CABG & 77 & $>54 \%$ & $52 \%$ & 2 & 30 & 60 & 31 & 6 & & \\
\hline & angiography. & & & $45 \%-54 \%$ & $22 \%$ & & & & & & & \\
\hline & & & & $35 \%-44 \%$ & $12 \%$ & & & & & & & \\
\hline & & & & $25 \%-34 \%$ & $5 \%$ & & & & & & & \\
\hline & & & & $<25 \%$ & $7 \%$ & & & & & & & \\
\hline \multirow[t]{2}{*}{ Syed et al ${ }^{\mathrm{E} 88}$} & None & OPCAB & 81 & $43 \%(25 \%-65 \%)$ & & NR & 48 & NR & NR & NR & NR & 0 \\
\hline & & CABG & 76 & $46 \%(25 \%-65 \%)$ & & & 55 & & & & & \\
\hline \multirow[t]{2}{*}{ Tang et al ${ }^{\mathrm{E} 9}$} & Age $>80 \mathrm{y}$, preexisting & OPCAB & 75 & $\mathrm{NR}$ & & 0 & 0 & NR & NR & NR & 0 & 0 \\
\hline & $\begin{array}{l}\text { renal disease } \mathrm{SCr} \\
>135 \mu \mathrm{mol} / \mathrm{L}, \mathrm{LVEF} \\
<40 \%, \text { chronic } \\
\text { uncontrolled } \\
\text { hypertension, DM, UA, } \\
\text { nephrotoxic drug use, } \\
\text { inotrope dependency }\end{array}$ & CABG & 85 & & & 0 & 0 & & & & & \\
\hline \multirow[t]{12}{*}{ Tatoulis et $\mathrm{al}^{\mathrm{E} 90}$} & Calcification or atheroma & OPCAB & 76 & $>60 \%$ & $56 \%$ & 4 & 36 & 80 & 36 & 6 & NR & 96 \\
\hline & of the ascending aorta, & & & $46 \%-59 \%$ & $32 \%$ & & & & & & & \\
\hline & extensive diffuse & & & $30 \%-46 \%$ & $12 \%$ & & & & & & & \\
\hline & coronary artery, deep & & & $<30 \%$ & $0 \%$ & & & & & & & \\
\hline & intramyocardial course & CABG & 80 & $>60 \%$ & $58 \%$ & 4 & 28 & 78 & 42 & 10 & & \\
\hline & of coronary arteries. & & & $46 \%-59 \%$ & $32 \%$ & & & & & & & \\
\hline & Patients operated on & & & $30 \%-46 \%$ & $10 \%$ & & & & & & & \\
\hline & urgently (ie, same day & & & $<30 \%$ & $0 \%$ & & & & & & & \\
\hline & as angiography) were & & & & & & & & & & & \\
\hline & excluded, because & & & & & & & & & & & \\
\hline & informed consent was & & & & & & & & & & & \\
\hline & not uniformly possible. & & & & & & & & & & & \\
\hline \multirow[t]{6}{*}{ Tully et $\mathrm{al}^{\mathrm{E} 91}$} & LVEF $<40 \%$; chronic & OPCAB & 81.2 & $>40 \%$ & $100 \%$ & NR & 13.3 & 56.7 & NR & NR & 0 & 0 \\
\hline & renal failure (SCr & CABG & & $>40 \%$ & $100 \%$ & & 22.2 & 58.3 & & & & \\
\hline & $\begin{array}{l}<0.3 \mathrm{mmol} / \mathrm{L}) \text {; COPD; } \\
>3 \text { distal anastomoses; }\end{array}$ & & & & & & & & & & & \\
\hline & emergency surgery; & & & & & & & & & & & \\
\hline & preoperative UA; recent & & & & & & & & & & & \\
\hline & $\begin{array}{l}\text { MI (within } 6 \mathrm{wk} \text { ); } \\
\text { participation in another }\end{array}$ & & & & & & & & & & & \\
\hline
\end{tabular}




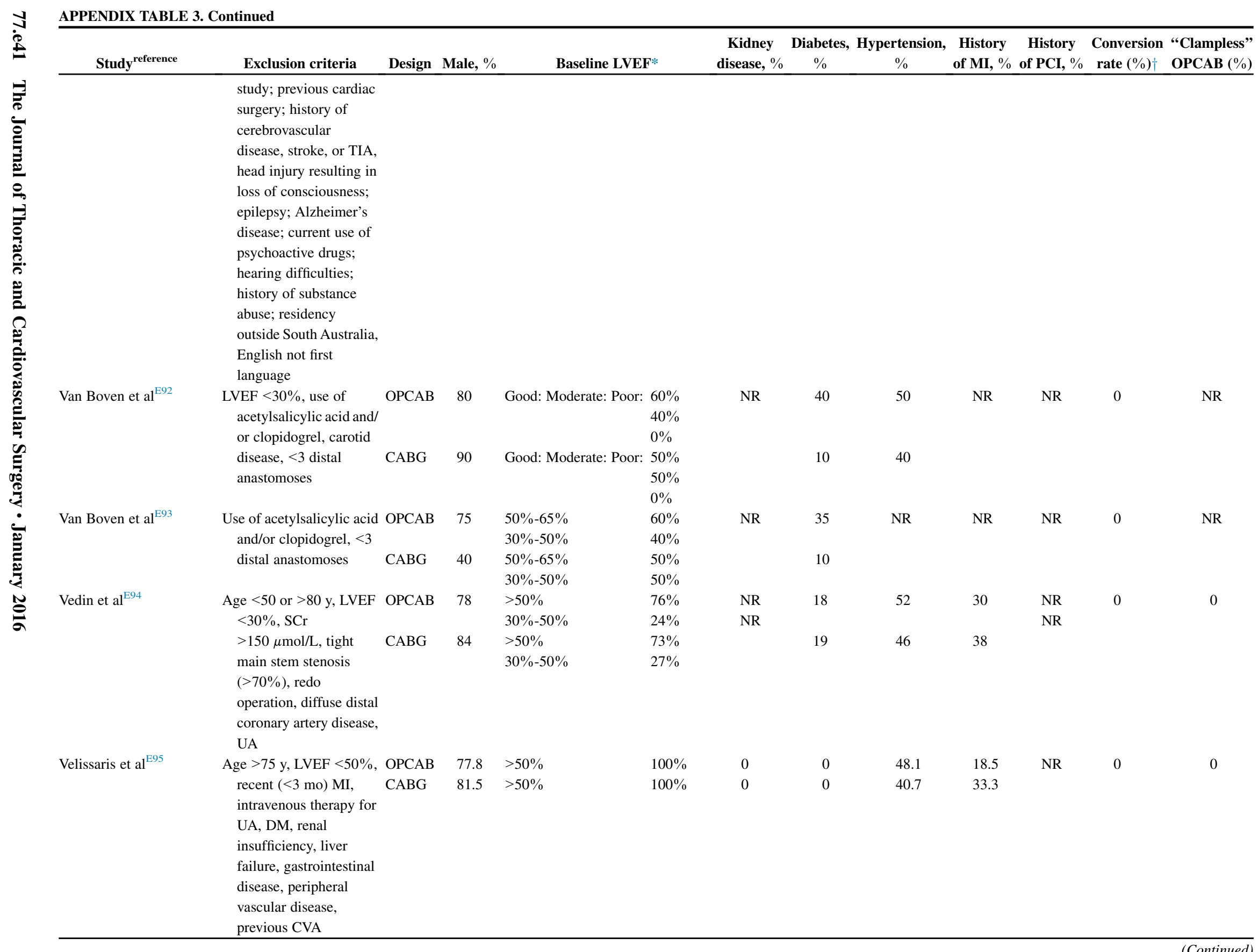




\begin{tabular}{|c|c|c|c|c|c|c|c|c|c|c|c|c|}
\hline Study ${ }^{\text {reference }}$ & Exclusion criteria & Design & Male, \% & Baselin & & $\begin{array}{c}\text { Kidney } \\
\text { disease, \% }\end{array}$ & $\begin{array}{c}\text { Diabetes, } \\
\%\end{array}$ & $\begin{array}{c}\text { Hypertension, } \\
\%\end{array}$ & $\begin{array}{c}\text { History } \\
\text { of MI, \% }\end{array}$ & $\begin{array}{c}\text { History } \\
\text { of PCI, \% }\end{array}$ & $\begin{array}{c}\text { Conversion } \\
\text { rate }(\%) \dagger\end{array}$ & $\begin{array}{l}\text { "Clampless" } \\
\text { OPCAB }(\%)\end{array}$ \\
\hline \multirow[t]{2}{*}{ Velissaris et $\mathrm{al}^{\mathrm{E} 96}$} & \multirow[b]{2}{*}{$\begin{array}{l}\text { Age }>75 \text { y, LVEF }<50 \% \text {, } \\
\text { recent }(<3 \text { mo) MI, } \\
\text { intravenous therapy for } \\
\text { UA, DM, renal } \\
\text { insufficiency, endocrine } \\
\text { disease, steroid therapy, } \\
\text { previous CVA }\end{array}$} & OPCAB & 76.9 & $>50 \%$ & $100 \%$ & 0 & 0 & 50 & 19.2 & \multirow[t]{2}{*}{ NR } & \multirow[t]{2}{*}{0} & \multirow[t]{2}{*}{0} \\
\hline & & CABG & 80.1 & $>50 \%$ & $100 \%$ & 0 & 0 & 50 & 34.6 & & & \\
\hline Vural et al ${ }^{\mathrm{E} 97}$ & NA & $\begin{array}{l}\text { OPCAB } \\
\text { CABG }\end{array}$ & NR & NR & & NR & NR & NR & NR & NR & 0 & NR \\
\hline \multirow[t]{2}{*}{ Wan et al ${ }^{\mathrm{E} 98}$} & \multirow[b]{2}{*}{$\begin{array}{l}\text { Recent MI, UA, steroid } \\
\text { treatment, age }>75 \mathrm{y}, \\
\mathrm{SCr}>200 \mu \mathrm{mol} / \mathrm{L}, \\
\text { single-vessel disease, } \\
\text { previous open cardiac } \\
\text { procedure, LVEF } \\
<30 \%\end{array}$} & OPCAB & 83.3 & \multirow{2}{*}{\multicolumn{2}{|c|}{$\begin{array}{l}52.93 \pm 13.58 \% \\
50.42 \pm 12.04 \%\end{array}$}} & \multirow[t]{2}{*}{ NR } & 38.9 & 72.2 & 22.2 & \multirow[t]{2}{*}{ NR } & \multirow[t]{2}{*}{0} & \multirow[t]{2}{*}{0} \\
\hline & & CABG & 57.9 & & & & 31.6 & 78.9 & 15.8 & & & \\
\hline Wandschneider et $\mathrm{al}^{\mathrm{E} 99}$ & $\begin{array}{l}\text { Valve operations, } \\
\text { combined procedures, } \\
\text { or emergency } \\
\text { operations; renal } \\
\text { insufficiency or any } \\
\text { kind of neurologic } \\
\text { symptoms }\end{array}$ & $\begin{array}{l}\text { OPCAB } \\
\text { CABG }\end{array}$ & $\begin{array}{l}78.0 \\
79.1\end{array}$ & NR & & NR & NR & NR & NR & NR & $21.15 \Phi$ & NR \\
\hline Wehlin et $\mathrm{al}^{\mathrm{E} 100}$ & $\begin{array}{l}\text { Age }<50 \text { or }>80 \text { y, LVEF } \\
\quad<30 \%, \text { SCr }\end{array}$ & OPCAB & 81 & $\begin{array}{l}>50 \% \\
30 \%-50 \%\end{array}$ & $\begin{array}{l}80 \% \\
19 \%\end{array}$ & NR & 14 & NR & NR & NR & 4.54 & 0 \\
\hline & $\begin{array}{l}>150 \mu \mathrm{mol} / \mathrm{L}, \mathrm{LM} \\
\text { stenosis, redo } \\
\text { operation, peripheral } \\
\text { CAD, UA, history of } \\
\text { cerebrovascular disease }\end{array}$ & CABG & 81 & $\begin{array}{l}>50 \% \\
30 \%-50 \%\end{array}$ & $\begin{array}{l}94 \% \\
6 \%\end{array}$ & & 19 & & & & & \\
\hline Wehlin et $\mathrm{al}^{\mathrm{E} 101}$ & $\begin{array}{l}\text { Age }<50 \text { or }>80 \text { y, LVEF } \\
\quad<30 \%, \text { SCr }\end{array}$ & OPCAB & 100 & $\begin{array}{l}>50 \% \\
30 \%-50 \%\end{array}$ & $\begin{array}{l}73 \% \\
27 \%\end{array}$ & NR & 18 & NR & NR & NR & 0 & 0 \\
\hline & $\begin{array}{l}>150 \mu \mathrm{mol} / \mathrm{L}, \mathrm{LM} \\
\text { stenosis, redo } \\
\text { operation, peripheral } \\
\text { CAD, UA, history of } \\
\text { cerebrovascular disease }\end{array}$ & CABG & 89 & $\begin{array}{l}>50 \% \\
30 \%-50 \%\end{array}$ & $\begin{array}{l}89 \% \\
11 \%\end{array}$ & & 11 & & & & & \\
\hline Yu et $\mathrm{al}^{\mathrm{E} 103}$ & Emergency or urgent & OPCAB & 78.4 & $43.39 \pm 9.53 \%$ & & NR & 49 & 60.9 & 70.6 & 17.6 & 3.92 & 0 \\
\hline & $\begin{array}{l}\text { operation, combined } \\
\text { valve surgery, }\end{array}$ & CABG & 74.5 & $41.9 \pm 8.8 \%$ & & & 41.2 & 68.6 & 76.5 & 15.6 & & \\
\hline
\end{tabular}




\begin{tabular}{|c|c|c|c|c|c|c|c|c|c|c|c|c|}
\hline Study ${ }^{\text {reference }}$ & Exclusion criteria & Design & Male, \% & Bas & & $\begin{array}{c}\text { Kidney } \\
\text { disease, \% }\end{array}$ & $\begin{array}{c}\text { Diabetes, } \\
\%\end{array}$ & $\begin{array}{c}\text { Hypertension, } \\
\%\end{array}$ & $\begin{array}{c}\text { History } \\
\text { of MI, \% }\end{array}$ & $\begin{array}{c}\text { History } \\
\text { of PCI, \% }\end{array}$ & $\begin{array}{c}\text { Conversion } \\
\text { rate }(\%) \dagger\end{array}$ & $\begin{array}{l}\text { "Clampless" } \\
\text { OPCAB (\%) }\end{array}$ \\
\hline Zamvar et al $^{\mathrm{E} 102}$ & $\begin{array}{l}\text { dyskinetic ventricles } \\
\text { (LV aneurysms), history } \\
\text { of renal insufficiency } \\
\text { ( } \mathrm{SCr}>2 \mathrm{mg} / \mathrm{dL} \text { ), stroke } \\
\text { or TIA within } 1 \mathrm{mo}, \\
\text { continuous infusion of } \\
\text { inotropics on the day of } \\
\text { the operation } \\
\text { Carotid artery stenosis } \\
>50 \% \text {, recent MI } \\
\text { (within } 1 \mathrm{mo} \text { ), previous } \\
\text { TIA or CVA, previous } \\
\text { psychiatric illness, } \\
\text { renal failure, } \\
\text { emergency operation, } \\
\text { reoperation, combined } \\
\text { valvular surgery }\end{array}$ & OPCAB & 90 & $\begin{array}{l}>50 \% \\
30 \%-50 \% \\
<30 \% \\
>50 \% \\
30 \%-50 \% \\
<30 \%\end{array}$ & $\begin{array}{l}76.6 \% \\
20 \% \\
3.3 \% \\
76.6 \% \\
16.6 \% \\
6.6 \%\end{array}$ & NR & NR & NR & NR & NR & 0 & 0 \\
\hline $\begin{array}{l}\angle V E F, \text { Left ventricle ej } \\
C A B G \text {, coronary artery } \\
\text { Surgery Trial; } B H A C A S \\
\text { aminotransferase; } U L N \\
\text { patients randomised to } \\
\text { transesophageal echoca } \\
\text { bypass grafting in elde } \\
\text { Investigation study; } P H \\
C A D \text {, Coronary artery } \\
\text { comparison between of } \\
\text { off-pump and on-pump } \\
\text { vessel Coronary Artery } \\
\text { arterial revascularizatio } \\
\text { root was done with the }\end{array}$ & $\begin{array}{l}\text { fraction; } M I \text {, myocardial infarct } \\
\text { ss graft; } D M \text {, diabetes mellitus; } \\
\text { eating Heart Against Cardiopleg } \\
\text { r limit of normal; CORONARY, } \\
\text { on-Pump surgery; EuroSCORE, } \\
\text { raphy; DOORS, Danish on-pump } \\
\text { tients; AIDS, acquired immunoc } \\
\text { lmonary hypertension; NSAID, } \\
\text { e; FPG, fasting plasma glucose; } \\
\text { p and on-pump surgery PRAGU } \\
\text { ry PRAGUE-11; NA, not availabl } \\
\text { iss Surgery; PD, posterior descer } \\
\text { apies. *Reported as in included } \\
\text { String II Proximal Seal System }\end{array}$ & $\begin{array}{l}\text { ion; } P C I, \mathrm{pe} \\
C V A, \text { cereb } \\
\text { ic Arrest St } \\
\text { 'ABG off or } \\
\text {, European s } \\
\text { versus off-1 } \\
\text { deficiency } \\
\text { nonsteroida } \\
\text {; FVC, forc } \\
\text { JE-4; } P R A G \\
\text { le; } I N R \text {, Inte } \\
\text { nding; } R O O \\
\text { studies. } † \mathrm{C} \\
\text { device (Ma }\end{array}$ & $\begin{array}{l}\text { ercutaneous } \\
\text { provascular } \\
\text { tudy } 1 ; B H A \\
\text { r on pump re } \\
\text { system for } \\
\text { pump rando } \\
\text { syndrome; } \\
\text { al anti-inflar } \\
\text { ced vital cap } \\
\text { GUE-6, Off- } \\
\text { ernational n } \\
\text { OBY, veterar } \\
\text { onversion } \mathrm{C} \\
\text { iquet AG, H }\end{array}$ & $\begin{array}{l}\text { coronary inter } \\
\text { accident; TIA, } \\
\text { CAS 2, Beatin } \\
\text { vascularization } \\
\text { ardiac operati } \\
\text { mization study } \\
\text { MIDCAB, min } \\
\text { nmatory drug; } \\
\text { acity; } F E V_{l}, \text { f } \\
\text { pump versus o } \\
\text { ormalized ratio } \\
\text { s affairs rando } \\
\text { PCAB to CAB } \\
\text { irrlingen, Gern }\end{array}$ & $\begin{array}{l}C A B \text {, off-p } \\
\text { nemic attac } \\
\text { inst Cardio } \\
\text { cardiopul } \\
\text { tion; } O N C \\
\text {-aortic ball } \\
\text { ive direct } \\
\text { s mellitus } \\
\text { tory volum } \\
\text { nary artery } \\
\text { vated partia } \\
\text { f bypass st } \\
\text { OPCAB o } \\
\text { not availa }\end{array}$ & $\begin{array}{l}\text { p coronary art } \\
U A, \text { unstable a } \\
\text { egic Arrest Stuc } \\
\text { nary resuscitat } \\
3 G \text {, on-pump cc } \\
\text { n pump; } A C S \text {, a } \\
\text { onary artery b } \\
M, \text { left main; } \\
\text { in } 1 \text { second; } A \Lambda \\
\text { ypass graft sur } \\
\text { hromboplastin } \\
\text { y; } G F R \text {, glome } \\
\text { rations were pe } \\
\text { e. }\end{array}$ & $\begin{array}{l}\text { tery bypass g } \\
\text { angina; } C r, \mathrm{c} \\
\text { Idy } 2 ; N Y H A, \\
\text { tion; } L C X, \mathrm{Le} \\
\text { oronary arter } \\
\text { acute corona } \\
\text { pypass; } B M I \text {, } \\
\text { MASS III, of } \\
M I \text {, acute my } \\
\text { gery in patie } \\
\text { time; } P R O M \\
\text { erular filtrati } \\
\text { erformed wit }\end{array}$ & $\begin{array}{l}\text { graft; } S C r \text {, serum cr } \\
\text { creatinine; } C O P D \text {, } \\
\text {, New York Heart } \\
\text { eft circumflex artery } \\
\text { ry bypass grafting; } \\
\text { rry syndrome; } L V, \text { le } \\
\text {, body mass index; } \\
\text { ff-pump and on-pu } \\
\text { yocardial infarctior } \\
\text { ents with EuroSCO } \\
\text { AISS, Prospective R } \\
\text { on rate; } M R I, \text { magr } \\
\text { th a side-biting clar }\end{array}$ & $\begin{array}{l}\text { reatinine; } C P L \\
\text { chronic pulm } \\
\text { Association; } A \\
\text { y; } C R I S P, \text { The } \\
O P C A B G \text {, of } \\
\text { eft ventricle; } C \\
\text {; JOCRI, Jap. } \\
\text { mp stable mu } \\
\text { n; } A F \text {, atrial fi } \\
\text { RE } \geq 6 ; P R A \\
\text { andomized C } \\
\text { netic resonanc } \\
\text { mp. §In all pa }\end{array}$ & $\begin{array}{l}B \text {, cardiopulm } \\
\text { nonary obstruc } \\
A S T \text {, aspartate } \\
\text { Coronary arte } \\
\text { ff-pump coron } \\
\text { GOPCABE; Gc } \\
\text { anese Off-Pun } \\
\text { ultivessel coro } \\
\text { fibrillation/flut } \\
\text { GUE-11, A ra } \\
\text {-omparison of } \\
\text { ce imaging; } S 1 \\
\text { atients, the pro }\end{array}$ & $\begin{array}{l}\text { onary bypass; } I \\
\text { ctive disease; } B \\
\text { aminotransfer; } \\
\text { ery bypass graft } \\
\text { ary artery bypa } \\
\text { ierman off-pum } \\
\text { mp Coronary R } \\
\text { onary artery by } \\
\text { tter; } P R A G U E- \\
\text { andomized com } \\
\text { f Off-Pump and } \\
\text { MART, surgica } \\
\text { oximal anastom }\end{array}$ & $\begin{array}{l}N R, \text { not reported; } \\
B B S, \text { Best Bypass } \\
\text { rase; } A L T \text {, alanine } \\
\text { fting in high-RISk } \\
\text { ass grafting; } T E E \text {, } \\
\text { np coronary artery } \\
\text { Revascularization } \\
\text { ypass graft study; } \\
-4, \text { A randomized } \\
\text { mparison between } \\
\text { d On-Pump Multi- } \\
\text { al management of } \\
\text { nosis in the aortic }\end{array}$ \\
\hline
\end{tabular}


APPENDIX TABLE 4. Potential sources of bias in included studies

\begin{tabular}{|c|c|c|c|c|c|c|c|}
\hline Study & $\begin{array}{c}\text { Random } \\
\text { sequence } \\
\text { generation } \\
\text { (selection bias) }\end{array}$ & $\begin{array}{c}\text { Allocation } \\
\text { concealment } \\
\text { (selection bias) }\end{array}$ & $\begin{array}{c}\text { Blinding of } \\
\text { participants } \\
\text { and personnel } \\
\text { (performance bias) }\end{array}$ & $\begin{array}{c}\text { Blinding of } \\
\text { outcome } \\
\text { assessment } \\
\text { (detection bias) }\end{array}$ & $\begin{array}{c}\text { Incomplete } \\
\text { outcome } \\
\text { data (attrition } \\
\text { bias) }\end{array}$ & $\begin{array}{l}\text { Selective } \\
\text { reporting } \\
\text { (reporting bias) }\end{array}$ & $\begin{array}{c}\text { Other } \\
\text { bias }\end{array}$ \\
\hline \multicolumn{8}{|l|}{$\overline{\text { Al-Ruzzeh et } \mathrm{al}^{\mathrm{E} 1}}$} \\
\hline \multicolumn{8}{|l|}{ Alwan et $\mathrm{al}^{\mathrm{E} 2}$} \\
\hline \multicolumn{8}{|l|}{ Ascione et $\mathrm{al}^{\mathrm{E} 3}$} \\
\hline \multicolumn{8}{|l|}{ Ascione et $\mathrm{al}^{\mathrm{E} 4}$} \\
\hline \multicolumn{8}{|l|}{ Baker et $\mathrm{al}^{\mathrm{E} 5}$} \\
\hline \multicolumn{8}{|c|}{ Moller et al $[\mathrm{BBS}]^{\mathrm{E} 6}$} \\
\hline \multicolumn{8}{|c|}{$\begin{array}{l}\text { Angelini et al } \\
\qquad[\text { BHACAS 1] }]^{\mathrm{E}, \mathrm{E} 8}\end{array}$} \\
\hline \multicolumn{8}{|l|}{$\begin{array}{l}\text { Angelini et al } \\
\qquad \text { [BHACAS 2] }^{\mathrm{E7}}\end{array}$} \\
\hline \multicolumn{8}{|l|}{ Bicer et $\mathrm{al}^{\mathrm{E} 9}$} \\
\hline \multicolumn{8}{|l|}{ Blacher et $\mathrm{al}^{\mathrm{E} 10}$} \\
\hline Bonacchi et $\mathrm{al}^{\mathrm{E} 11}$ & & & & & & & \\
\hline
\end{tabular}




\begin{tabular}{|c|c|c|c|c|c|c|c|}
\hline Study & $\begin{array}{c}\text { Random } \\
\text { sequence } \\
\text { generation } \\
\text { (selection bias) }\end{array}$ & $\begin{array}{c}\text { Allocation } \\
\text { concealment } \\
\text { (selection bias) }\end{array}$ & $\begin{array}{c}\text { Blinding of } \\
\text { participants } \\
\text { and personnel } \\
\text { (performance bias) }\end{array}$ & $\begin{array}{c}\text { Blinding of } \\
\text { outcome } \\
\text { assessment } \\
\text { (detection bias) }\end{array}$ & $\begin{array}{c}\text { Incomplete } \\
\text { outcome } \\
\text { data (attrition } \\
\text { bias) }\end{array}$ & $\begin{array}{c}\text { Selective } \\
\text { reporting } \\
\text { (reporting bias) }\end{array}$ & $\begin{array}{c}\text { Other } \\
\text { bias }\end{array}$ \\
\hline \multicolumn{8}{|l|}{ Caputo et $\mathrm{al}^{\mathrm{E} 12}$} \\
\hline \multicolumn{8}{|l|}{ Carrier et $\mathrm{al}^{\mathrm{E} 13}$} \\
\hline \multicolumn{8}{|l|}{ Cavalca et $\mathrm{al}^{\mathrm{E} 14}$} \\
\hline \multicolumn{8}{|c|}{ Chowdhury et $\mathrm{al}^{\mathrm{E} 15}$} \\
\hline \multicolumn{8}{|c|}{ Lamy et al [CORONARY] ${ }^{\mathrm{E} 16}$} \\
\hline \multicolumn{8}{|l|}{ Covino et $\mathrm{al}^{\mathrm{E} 17}$} \\
\hline \multicolumn{8}{|c|}{ Rogers et al [CRISP] ${ }^{\mathrm{E} 18}$} \\
\hline \multicolumn{8}{|l|}{ Czerny et $\mathrm{al}^{\mathrm{E} 19}$} \\
\hline \multicolumn{8}{|l|}{ Czerny et al ${ }^{\mathrm{E} 20}$} \\
\hline \multicolumn{8}{|l|}{ Diegeler et $\mathrm{al}^{\mathrm{E} 21}$} \\
\hline Dorman et $\mathrm{al}^{\mathrm{E} 22}$ & & & & & & & \\
\hline
\end{tabular}




\begin{tabular}{|c|c|c|c|c|c|c|c|}
\hline Study & $\begin{array}{c}\text { Random } \\
\text { sequence } \\
\text { generation } \\
\text { (selection bias) }\end{array}$ & $\begin{array}{c}\text { Allocation } \\
\text { concealment } \\
\text { (selection bias) }\end{array}$ & $\begin{array}{c}\text { Blinding of } \\
\text { participants } \\
\text { and personnel } \\
\text { (performance bias) }\end{array}$ & $\begin{array}{c}\text { Blinding of } \\
\text { outcome } \\
\text { assessment } \\
\text { (detection bias) }\end{array}$ & $\begin{array}{c}\text { Incomplete } \\
\text { outcome } \\
\text { data (attrition } \\
\text { bias) }\end{array}$ & $\begin{array}{c}\text { Selective } \\
\text { reporting } \\
\text { (reporting bias) }\end{array}$ & $\begin{array}{c}\text { Other } \\
\text { bias }\end{array}$ \\
\hline \multicolumn{8}{|c|}{ Houlind et al [DOORS] ${ }^{\mathrm{E} 23}$} \\
\hline \multicolumn{8}{|l|}{ Fattouch et $\mathrm{al}^{\mathrm{E} 24}$} \\
\hline \multicolumn{8}{|l|}{ Formica et $\mathrm{al}^{\mathrm{E} 25}$} \\
\hline \multicolumn{8}{|l|}{ Gasz et $\mathrm{al}^{\mathrm{E} 26}$} \\
\hline \multicolumn{8}{|l|}{ Gasz et al $^{\mathrm{E} 27}$} \\
\hline \multicolumn{8}{|l|}{ Gerola et al ${ }^{\mathrm{E} 28}$} \\
\hline \multicolumn{8}{|l|}{ Gu et $\mathrm{al}^{\mathrm{E} 29}$} \\
\hline \multicolumn{8}{|l|}{ Guler et $\mathrm{al}^{\mathrm{E} 30}$} \\
\hline \multicolumn{8}{|l|}{ Gonenc et al ${ }^{\mathrm{E} 31}$} \\
\hline \multicolumn{8}{|c|}{ Diegeler et al [GOPCABE] ${ }^{\mathrm{E} 32}$} \\
\hline Gulielmos et $\mathrm{al}^{\mathrm{E} 33}$ & & & & & & & \\
\hline
\end{tabular}




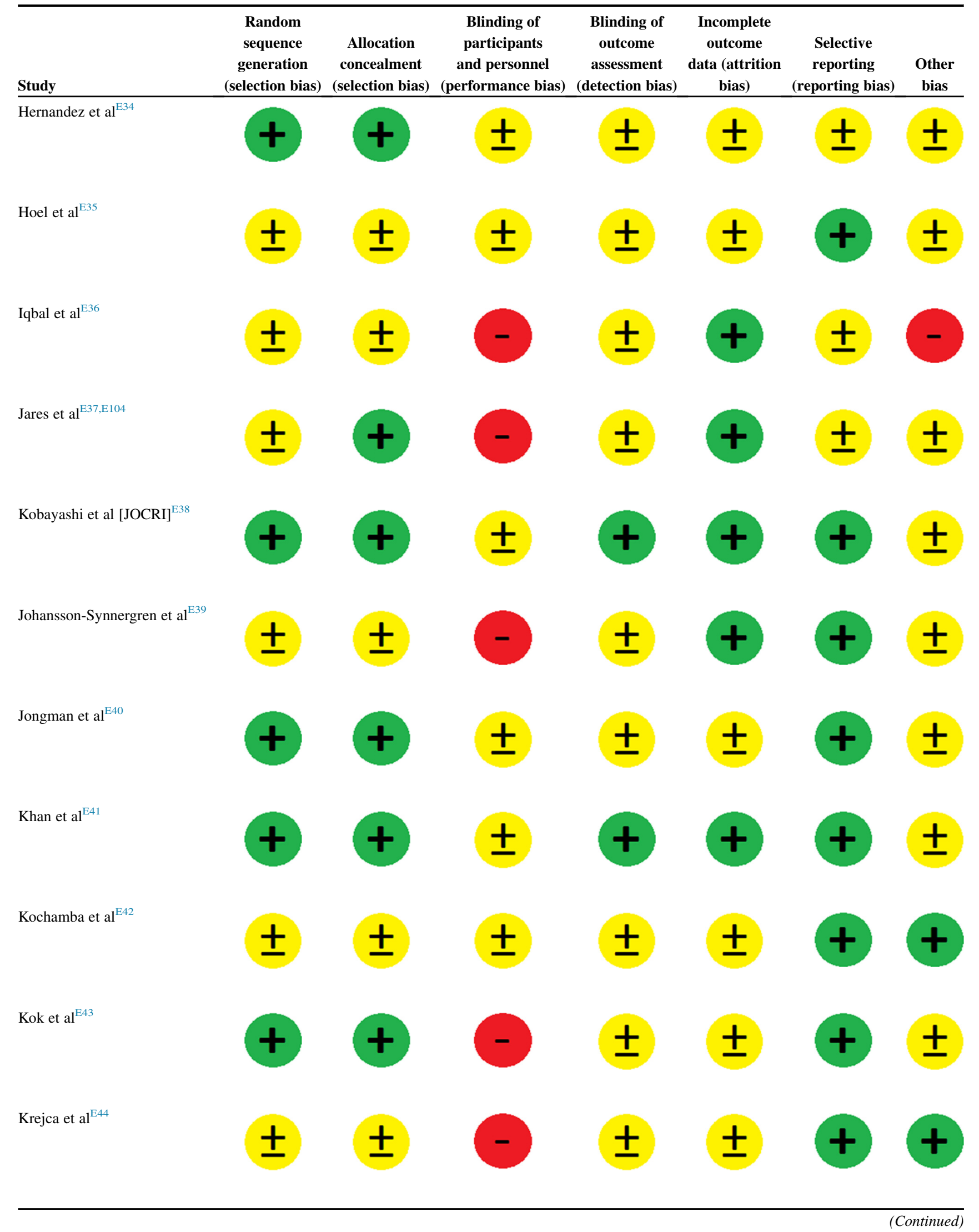




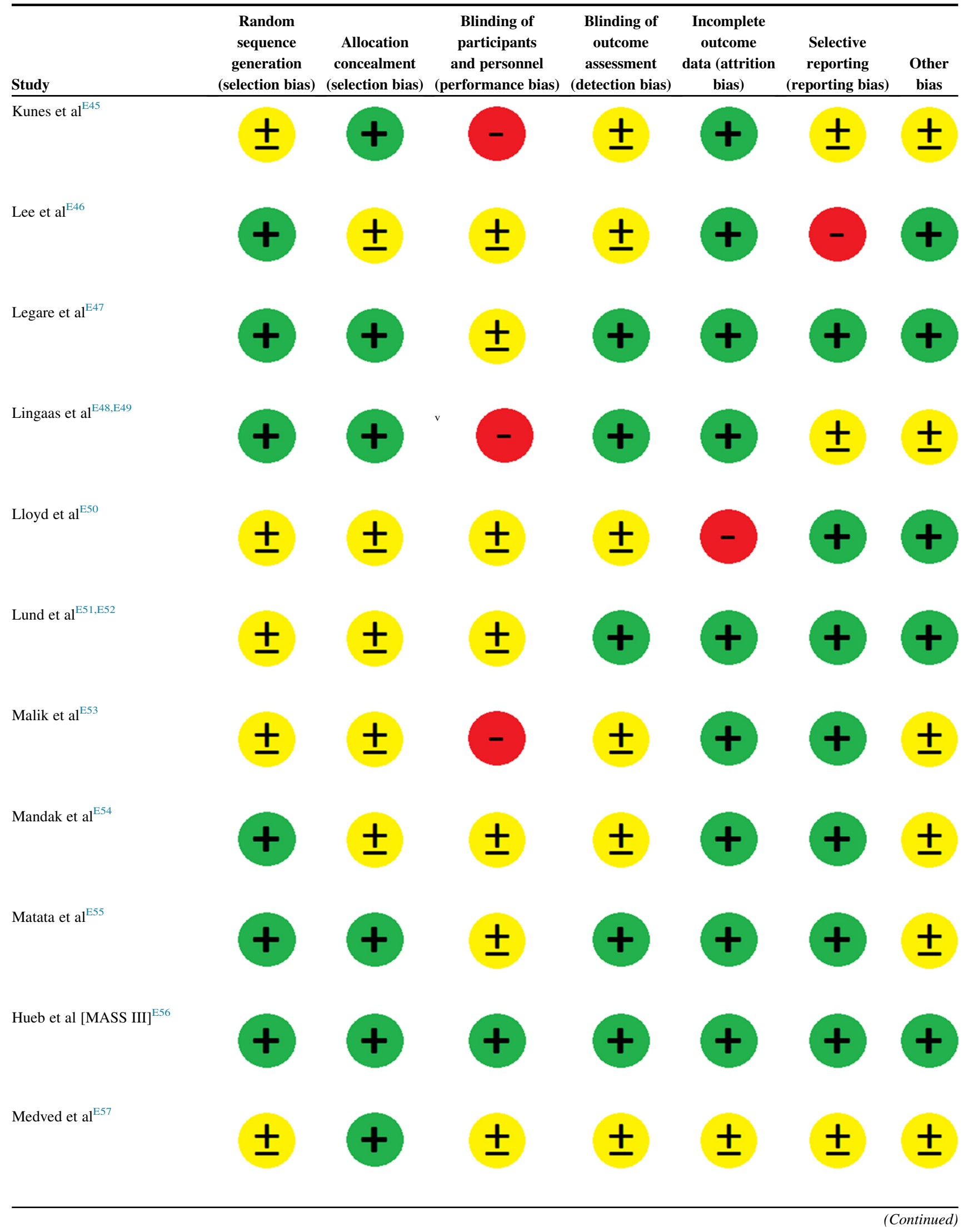




\begin{tabular}{|c|c|c|c|c|c|c|c|}
\hline Study & $\begin{array}{c}\text { Random } \\
\text { sequence } \\
\text { generation } \\
\text { (selection bias) }\end{array}$ & $\begin{array}{c}\text { Allocation } \\
\text { concealment } \\
\text { (selection bias) }\end{array}$ & $\begin{array}{c}\text { Blinding of } \\
\text { participants } \\
\text { and personnel } \\
\text { (performance bias) }\end{array}$ & $\begin{array}{c}\text { Blinding of } \\
\text { outcome } \\
\text { assessment } \\
\text { (detection bias) }\end{array}$ & $\begin{array}{c}\text { Incomplete } \\
\text { outcome } \\
\text { data (attrition } \\
\text { bias) }\end{array}$ & $\begin{array}{c}\text { Selective } \\
\text { reporting } \\
\text { (reporting bias) }\end{array}$ & $\begin{array}{c}\text { Other } \\
\text { bias }\end{array}$ \\
\hline \multicolumn{8}{|c|}{ Michaux et al ${ }^{\mathrm{E} 58, \mathrm{E} 59}$} \\
\hline \multicolumn{8}{|l|}{ Modine et $\mathrm{al}^{\mathrm{E} 60}$} \\
\hline \multicolumn{8}{|c|}{ Motallebzadeh et $\mathrm{al}^{\mathrm{E} 61}$} \\
\hline \multicolumn{8}{|c|}{ Motallebzadeh et $\mathrm{al}^{\mathrm{E} 62}$} \\
\hline \multicolumn{8}{|c|}{ Muneretto et $\mathrm{al}^{\mathrm{E} 63}$} \\
\hline \multicolumn{8}{|l|}{ Nesher et $\mathrm{al}^{\mathrm{E} 64}$} \\
\hline \multicolumn{8}{|l|}{ Niranjan et al ${ }^{\mathrm{E} 65}$} \\
\hline \multicolumn{8}{|c|}{ Nathoe et al [Octopus] ${ }^{\mathrm{E} 66}$} \\
\hline \multicolumn{8}{|c|}{ Lemma et al [On-Off] ${ }^{\mathrm{E} 67}$} \\
\hline \multicolumn{8}{|l|}{ Onorati et $\mathrm{al}^{\mathrm{E} 8}$} \\
\hline Ozkara et $\mathrm{al}^{\mathrm{E} 69}$ & & & & & & & \\
\hline
\end{tabular}




\begin{tabular}{|c|c|c|c|c|c|c|c|}
\hline Study & $\begin{array}{c}\text { Random } \\
\text { sequence } \\
\text { generation } \\
\text { (selection bias) }\end{array}$ & $\begin{array}{c}\text { Allocation } \\
\text { concealment } \\
\text { (selection bias) }\end{array}$ & $\begin{array}{c}\text { Blinding of } \\
\text { participants } \\
\text { and personnel } \\
\text { (performance bias) }\end{array}$ & $\begin{array}{c}\text { Blinding of } \\
\text { outcome } \\
\text { assessment } \\
\text { (detection bias) }\end{array}$ & $\begin{array}{c}\text { Incomplete } \\
\text { outcome } \\
\text { data (attrition } \\
\text { bias) }\end{array}$ & $\begin{array}{c}\text { Selective } \\
\text { reporting } \\
\text { (reporting bias) }\end{array}$ & $\begin{array}{c}\text { Other } \\
\text { bias }\end{array}$ \\
\hline \multicolumn{8}{|c|}{ Paparella et al ${ }^{\mathrm{E} 70}$} \\
\hline \multicolumn{8}{|c|}{ Parolari et $\mathrm{al}^{\mathrm{E} 71}$} \\
\hline \multicolumn{8}{|c|}{ Parolari et $\mathrm{al}^{\mathrm{E} 72}$} \\
\hline \multicolumn{8}{|c|}{ Penttilä et al ${ }^{\mathrm{E} 73}$} \\
\hline \multicolumn{8}{|c|}{ Straka et al [PRAGUE-4] ${ }^{\mathrm{E} 74}$} \\
\hline \multicolumn{8}{|c|}{ Hlavicka et al [PRAGUE-6] ${ }^{\mathrm{E} 75}$} \\
\hline \multicolumn{8}{|c|}{ Bednar et al [PRAGUE 11] ${ }^{\mathrm{E} 76}$} \\
\hline \multicolumn{8}{|c|}{ Sousa Uva et al [PROMISS] ${ }^{\mathrm{E} 77}$} \\
\hline \multicolumn{8}{|c|}{ 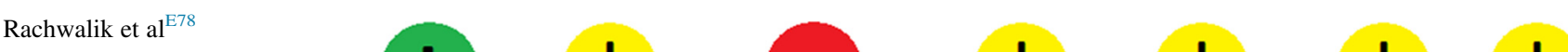 } \\
\hline \multicolumn{8}{|l|}{ Rainio et al ${ }^{\mathrm{E} 79}$} \\
\hline Raja et $\mathrm{al}^{\mathrm{E} 80}$ & & & & & & & \\
\hline
\end{tabular}




\begin{tabular}{|c|c|c|c|c|c|c|c|}
\hline Study & $\begin{array}{c}\text { Random } \\
\text { sequence } \\
\text { generation } \\
\text { (selection bias) } \\
\end{array}$ & $\begin{array}{c}\text { Allocation } \\
\text { concealment } \\
\text { (selection bias) } \\
\end{array}$ & $\begin{array}{c}\text { Blinding of } \\
\text { participants } \\
\text { and personnel } \\
\text { (performance bias) }\end{array}$ & $\begin{array}{c}\text { Blinding of } \\
\text { outcome } \\
\text { assessment } \\
\text { (detection bias) } \\
\end{array}$ & $\begin{array}{c}\text { Incomplete } \\
\text { outcome } \\
\text { data (attrition } \\
\text { bias) } \\
\end{array}$ & $\begin{array}{c}\text { Selective } \\
\text { reporting } \\
\text { (reporting bias) } \\
\end{array}$ & $\begin{array}{c}\text { Other } \\
\text { bias }\end{array}$ \\
\hline \multicolumn{8}{|c|}{ Rasmussen et $\mathrm{al}^{\mathrm{E} 81}$} \\
\hline \multicolumn{8}{|l|}{ Rastan et $\mathrm{al}^{\mathrm{E} 82}$} \\
\hline \multicolumn{8}{|c|}{ Schroyer et al [ROOBY] $]^{\mathrm{E} 83}$} \\
\hline \multicolumn{8}{|c|}{ Sahlman et al ${ }^{\mathrm{E} 84}$} \\
\hline \multicolumn{8}{|c|}{ Sajja LR et al ${ }^{\mathrm{E} 85}$} \\
\hline \multicolumn{8}{|c|}{ Selvanayagam et al ${ }^{\mathrm{E} 86}$} \\
\hline \multicolumn{8}{|c|}{ Puskas et al [SMART] ${ }^{\mathrm{E} 87}$} \\
\hline \multicolumn{8}{|l|}{ Syed et $\mathrm{al}^{\mathrm{E} 88}$} \\
\hline \multicolumn{8}{|l|}{ Tang et $\mathrm{al}^{\mathrm{E} 89}$} \\
\hline \multicolumn{8}{|c|}{ Tatoulis et al ${ }^{\mathrm{E} 90}$} \\
\hline Tully et $\mathrm{al}^{\mathrm{E} 91}$ & & & & & & & \\
\hline
\end{tabular}




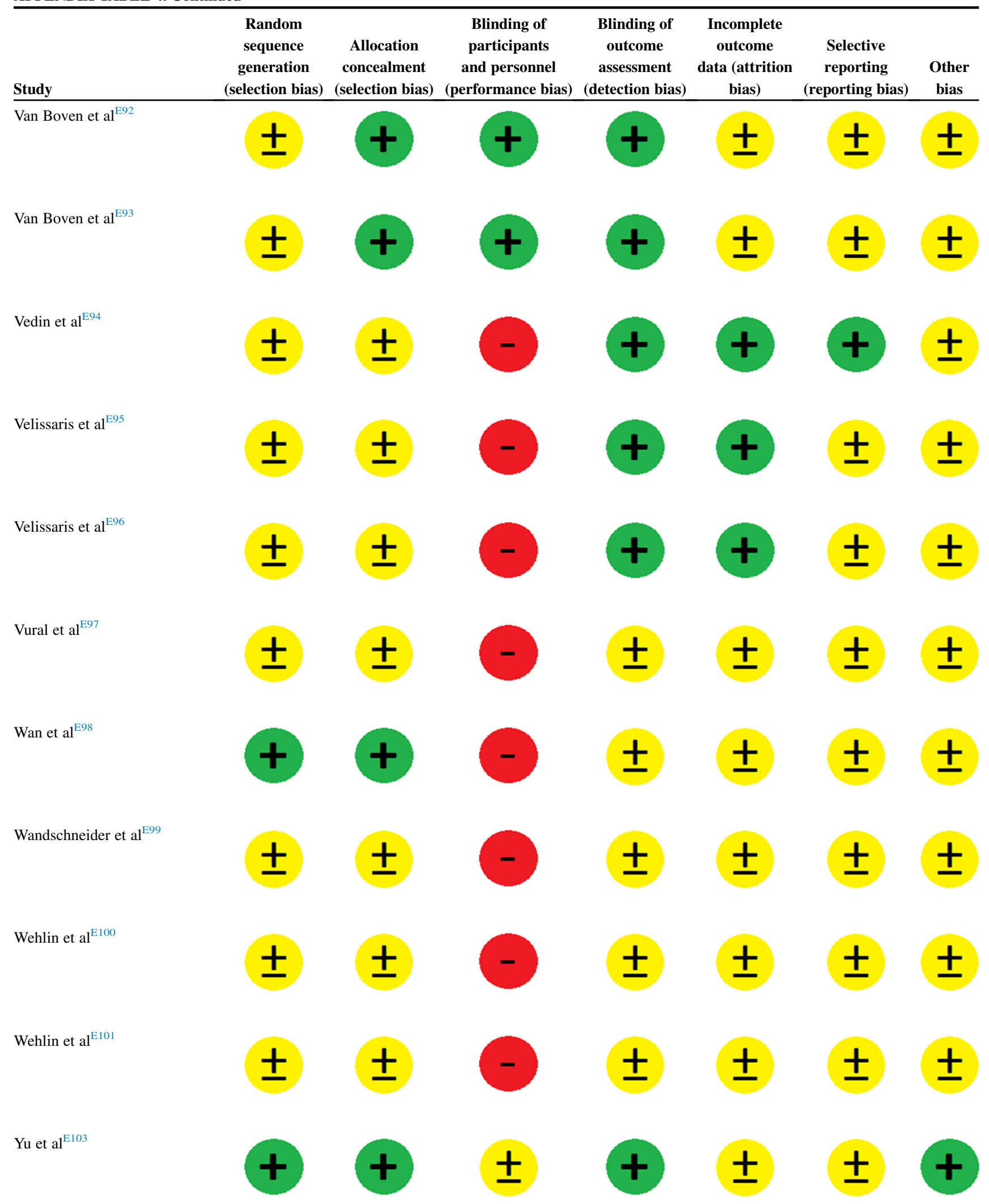


APPENDIX TABLE 4. Continued

\begin{tabular}{|c|c|c|c|c|c|c|c|}
\hline Study & $\begin{array}{c}\text { Random } \\
\text { sequence } \\
\text { generation } \\
\text { (selection bias) }\end{array}$ & $\begin{array}{c}\text { Allocation } \\
\text { concealment } \\
\text { (selection bias) }\end{array}$ & $\begin{array}{c}\text { Blinding of } \\
\text { participants } \\
\text { and personnel } \\
\text { (performance bias) }\end{array}$ & $\begin{array}{c}\text { Blinding of } \\
\text { outcome } \\
\text { assessment } \\
\text { (detection bias) }\end{array}$ & $\begin{array}{c}\text { Incomplete } \\
\text { outcome } \\
\text { data (attrition } \\
\text { bias) }\end{array}$ & $\begin{array}{c}\text { Selective } \\
\text { reporting } \\
\text { (reporting bias) }\end{array}$ & $\begin{array}{c}\text { Other } \\
\text { bias }\end{array}$ \\
\hline \multicolumn{8}{|l|}{ Zamvar et al ${ }^{\mathrm{E} 102}$} \\
\hline Cohen's kappa & 0.78 & 0.81 & 0.76 & 0.76 & 0.80 & 0.65 & 0.74 \\
\hline
\end{tabular}


APPENDIX TABLE 5. Endpoint definitions

Endpoint definitions

\begin{tabular}{cc} 
Study & Cerebral stroke \\
\hline Al-Ruzzeh et $\mathrm{al}^{\mathrm{El}}$ & Focal neurologic signs and symptoms persisting for
\end{tabular}

$$
>24 \mathrm{~h} \text {, confirmed by CT }
$$

Alwan et $\mathrm{al}^{\mathrm{E} 2}$

Not defined

Ascione et $\mathrm{al}^{\mathrm{E} 3}$
Ascione et $\mathrm{al}^{\mathrm{E} 4}$
Baker et $\mathrm{al}^{\mathrm{E} 5}$
Moller et al $[\mathrm{BBS}]^{\mathrm{E} 6}$

Angelini et al [BHACAS 1] $]^{\mathrm{E} 7, \mathrm{E} 8}$

Angelini et al [BHACAS 2] ${ }^{\mathrm{E} 7}$

Bicer et al ${ }^{\mathrm{E} 9}$

Blacher et al ${ }^{\mathrm{E} 10}$

Bonacchi et $\mathrm{al}^{\mathrm{E} 11}$

Caputo et $\mathrm{al}^{\mathrm{E} 12}$

Carrier et $\mathrm{al}^{\mathrm{E} 13}$

Cavalca et $\mathrm{al}^{\mathrm{E} 14}$

Chowdhury et $\mathrm{al}^{\mathrm{E} 15}$

Lamy et al [CORONARY] ${ }^{\mathrm{E} 16}$

Not defined
Adjudicated by a CEC

Not defined

Not defined

Not defined

Global or focal neurologic deficits persisting for $>24 \mathrm{~h}$ and verified by a neurologist or brain CT scan

Adjudicated by a CEC

Not defined

Evidence in the postoperative period of a new central neurologic deficit persisting for $>72 \mathrm{~h}$

Adjudicated by a CEC

Adjudicated by a CEC

Not defined

Not defined

Acute focal neurologic deficit thought to be of vascular origin persisting for $>24 \mathrm{~h}$ and confirmed by a neurologist

\section{Myocardial infarction}

New Q-waves on ECG, CK-MB concentration $>50 \mathrm{ng} / \mathrm{mL}$ with ECG changes or $>70 \mathrm{ng} / \mathrm{mL}$ without ECG changes

New Q-waves $>0.04 \mathrm{~ms}$ and a reduction in R-waves of $>25 \%$ in at least 2 leads. cTnI diagnostic criteria for MI: cTnI peak concentration $>3.7 \mu \mathrm{g} / \mathrm{L}$ and $\mathrm{cTnI}$ concentration $>3.1 \mu \mathrm{g} / \mathrm{L}$ at $12 \mathrm{~h}$ or $>2.5 \mu \mathrm{g} / \mathrm{L}$ at $24 \mathrm{~h}$

Not defined

Not defined

Not defined

CK-MB increase $>80 \mu \mathrm{g} / \mathrm{L}$ or $\mathrm{TnT}>3.0 \mu \mathrm{g} / \mathrm{L}$ the first $48 \mathrm{~h}$ after surgery; thereafter, the definition was enzymatic elevation of CK-MB $>10 \mu \mathrm{g} / \mathrm{L}$ or $\mathrm{TnT}>0.1 \mu \mathrm{g} / \mathrm{L}$ along with at least 1 of the following findings: classic angina symptoms, ECG signs of necrosis or ischemia, or coronary reintervention

New Q-waves of $>0.04 \mathrm{~ms}$ and/or a reduction in Rwaves of $>25 \%$ in $\geq 2$ leads

New Q-waves of $>0.04 \mathrm{~ms}$ and/or a reduction in Rwaves of $>25 \%$ in $\geq 2$ leads

Not defined

New Q-waves, reduction of $>25 \%$ in R-wave amplitude in $\geq 2$ contiguous leads, CK-MB level $\geq 5 \%$ of the creatine phosphate kinase value for $>18 \mathrm{~h}$

Not defined

New Q-waves of $>0.04 \mathrm{~ms}$, or a reduction in R-waves of $>25 \%$ in at least 2 leads

Serial CK-MB level $>100$ U or new Q-wave on postoperative ECG

Not defined

One of the following criteria: (1) appearance of a new and persistent Q-wave with a duration of $>40 \mathrm{~ms}$ in at least 2 adjacent leads; (2) disappearance of an $\mathrm{R}$-wave or a $25 \%$ reduction of $\mathrm{R}$-waves in 2 leads; (3) appearance of ST-segment deviations at the Jpoint in 2 or more contiguous leads with cutoff points $>0.2 \mathrm{mV}$ in leads $\mathrm{V} 1, \mathrm{~V} 2$, and $\mathrm{V} 3$ and $>0.1 \mathrm{mV}$ in other leads or T-wave abnormalities in 2 or more continuous leads; (4) appearance of a new-onset LBBB; (5) new and persistent wallmotion abnormalities on TEE; and (6) requirement of inotropic support to maintain stable hemodynamics as stated in the absence of mechanical external compression

Perioperative MI (within $72 \mathrm{~h}$ of surgery): CK-MB measurement $\geq 5$ times the 99 th percentile upper reference limit without new pathological Q-waves or new LBBB (non-Q-wave MI) or with new pathological Q-waves or new LBBB (Q-wave MI); angiographic evidence of new graft or native 


\section{Study}

Covino et $\mathrm{al}^{\mathrm{E} 17}$

Rogers et al [CRISP] ${ }^{\mathrm{E} 18}$

Czerny et $\mathrm{al}^{\mathrm{E} 19}$

Czerny et $\mathrm{al}^{\mathrm{E} 20}$

Diegeler et $\mathrm{al}^{\mathrm{E} 21}$

Dorman et al ${ }^{\mathrm{E} 22}$

Houlind et al [DOORS] $]^{\text {E23 }}$

Fattouch et $\mathrm{al}^{\mathrm{E} 24}$

Formica et al ${ }^{\mathrm{E} 25}$

Gasz et $\mathrm{al}^{\mathrm{E} 26}$

Gasz et al ${ }^{\mathrm{E} 27}$

Gerola et $\mathrm{al}^{\mathrm{E} 28}$

$\mathrm{Gu}$ et al ${ }^{\mathrm{E} 29}$

Guler et al ${ }^{\mathrm{E} 30}$

Gonenc et al ${ }^{\mathrm{E} 31}$

Diegeler et al [GOPCABE] $]^{\mathrm{E} 32}$

\section{Not defined}

New acute focal neurologic deficit thought to be of vascular origin, with signs or symptoms persisting $>24 \mathrm{~h}$ and confirmed by a neurologist

\author{
Adjudicated by a CEC \\ Adjudicated by a CEC \\ Not defined \\ Not defined \\ Acute focal or global neurologic deficit lasting $>24 \mathrm{~h}$ \\ or leading to death of presumed vascular origin
}

Not defined

Not defined

Not defined

Not defined

Neurologic dysfunction, including all alterations of behavior, mental confusion, and psychomotor agitation up to stroke and coma

Not defined

Not defined

Adjudicated by a CEC

New acute focal neurologic deficit thought to be of vascular origin with signs or symptoms lasting $>24 \mathrm{~h}$; stroke needs to be confirmed by a neurologist; imaging is encouraged to distinguish ischemic and hemorrhagic stroke. coronary artery occlusion; or imaging evidence of new loss of viable myocardium

Nonperioperative MI (later than $72 \mathrm{~h}$ after surgery): detection of a rise and/or fall of cardiac biomarkers with at least 1 value above the 99th percentile of the upper reference limit together with evidence of myocardial ischemia with at least 1 of the following: symptoms of ischemia, ECG changes indicative of new ischemia (new ST-T changes or new LBBB), development of pathological Q-

waves on the ECG, or imaging evidence of new loss of viable myocardium or new regional wall motion abnormality

Not defined

TnI level of $>0.5 \mu \mathrm{g} / \mathrm{L}$ or TnT level of $>0.2 \mu \mathrm{g} / \mathrm{L}$ and new pathological Q-waves with documented new wall motion abnormalities except in the septum; CK-MB level $\geq 10$ times the ULN (non-Q MI); or ECG changes consistent with infarction (new significant Q-waves $\geq 0.04 \mathrm{~cm}$ or a reduction in $\mathrm{R}$ waves of $>25 \%$, in at least 2 contiguous leads).

Adjudicated by a CEC

Adjudicated by a CEC

Not defined

Not defined

Postoperative acute MI: peak CK-MB of at least $75 \mathrm{ng} / \mathrm{mL}$ by day 3; new Q-wave evidence of MI, along with CK-MB of at least $50 \mathrm{ng} / \mathrm{mL}$ by day 3 ; new Q-wave evidence of MI by day 30 that was not present at day 3; MI (Q-wave or non-Q-wave) as defined according to World Health Organization criteria

ECG signs (Q-waves and ST-segment elevation) and increased serum myocardial enzyme levels (creatine phosphokinase and cardiac troponin I)

New Q-waves of $>0.05 \mathrm{mV}$ and a reduction in $\mathrm{R}$ waves of $>25 \%$ in at least 2 ECG leads, as well as new echocardiographic akinetic or hypokinetic areas

Not defined

Not defined

CK-MB level increase $\geq 5$ times the ULN.

\section{Not defined}

Not defined

Adjudicated by a CEC

Within $72 \mathrm{~h}$, at least 1 of the following: measured CK-MB $\geq 5$ times the 99 th percentile upper reference limit; angiographic evidence of new graft or native coronary artery occlusion; imaging evidence of new loss of viable myocardium later than $72 \mathrm{~h}$; and at least 1 of the following: 
Endpoint definitions

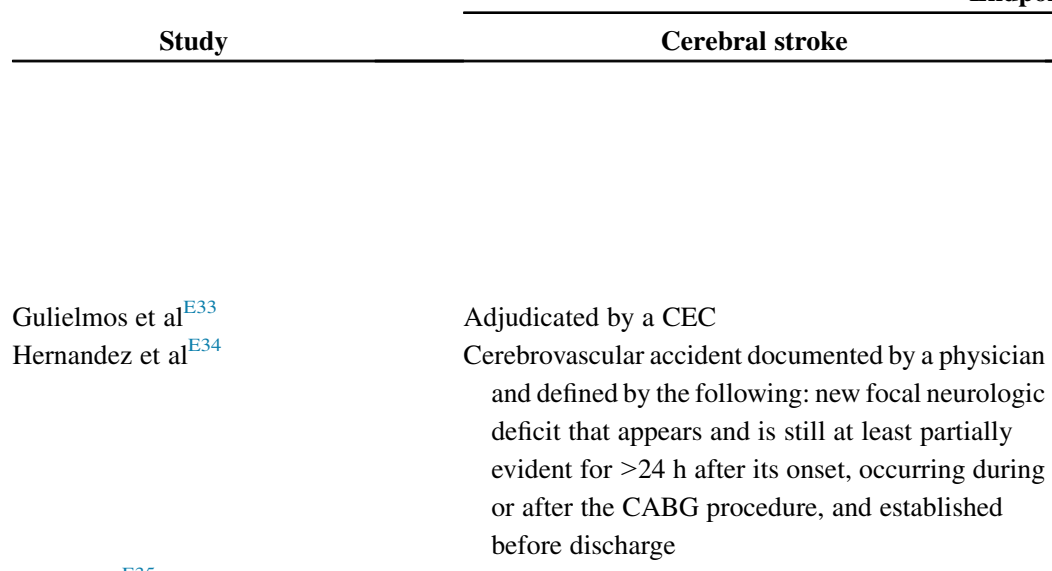

Hoel et $\mathrm{al}^{\mathrm{E} 35}$

Iqbal et al ${ }^{\mathrm{E} 36}$

Jares et al ${ }^{\mathrm{E} 37, \mathrm{E} 104}$

Kobayashi et al [JOCRI] ${ }^{\mathrm{E} 38}$

Johansson-Synnergren et $\mathrm{al}^{\mathrm{E} 39}$

Jongman et al ${ }^{\mathrm{E} 40}$

Khan et $\mathrm{al}^{\mathrm{E} 41}$

Kochamba et $\mathrm{al}^{\mathrm{E} 42}$

Kok et $\mathrm{al}^{\mathrm{E} 43}$

Krejca et $\mathrm{al}^{\mathrm{E} 44}$

Kunes et al ${ }^{\mathrm{E} 45}$

Lee et $\mathrm{al}^{\mathrm{E} 46}$

Legare et $\mathrm{al}^{\mathrm{E} 47}$

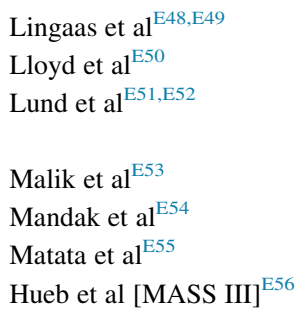

Medved et al ${ }^{\mathrm{E} 57}$

Michaux et al ${ }^{\mathrm{E} 58, \mathrm{E} 59}$

Modine et $\mathrm{al}^{\mathrm{E} 60}$

Motallebzadeh et $\mathrm{al}^{\mathrm{E} 61}$

Motallebzadeh et $\mathrm{al}^{\mathrm{E} 62}$

Muneretto et $\mathrm{al}^{\mathrm{E} 63}$

Nesher et $\mathrm{al}^{\mathrm{E} 64}$

\section{Not defined}

Any neurologic deficit persisting for $>30 \mathrm{~min}$

Not defined

Not defined

Adjudicated by a CEC

Not defined

Adjudicated by a CEC

Adjudicated by a CEC

Not defined

Not defined

Not defined

Adjudicated by a neurologist according to the modified National Institutes of Health Stroke Scale

Adjudicated by a CEC

\author{
Adjudicated by a CEC \\ Not defined \\ Neurologic deficit confirmed by MRI and neurologist \\ examination \\ Adjudicated by a CEC \\ Adjudicated by a CEC \\ Not defined \\ Focal brain injury persisting for $24 \mathrm{~h}$, combined with \\ an increase in disability of at least 1 grade on the \\ Rankin Scale
}

\author{
Not defined \\ Not defined \\ Not defined \\ Maximum CK-MB > 100 IU/L with ECG change \\ (new Q-wave or T-wave inversion by Minnesota \\ criteria code) \\ Adjudicated by a CEC \\ Not defined \\ Adjudicated by a CEC \\ Adjudicated by a CEC \\ Not defined \\ Not defined \\ Not defined \\ Adjudicated by a CEC
}

New Q-wave $(0.04 \mathrm{~ms})$ in at least 2 leads or ST changes on ECG in association with significant CK-MB enzyme release

Adjudicated by a CEC

Not defined

Not defined

ECG criteria or in-hospital death Adjudicated by a CEC

Not defined

Elevation of CK-MB $\geq 5$ times the ULN ( $>30 \mathrm{ng}$ / $\mathrm{mL}$ ) or TnI values $>5 \mathrm{ng} / \mathrm{mL}$ associated or not with the appearance of new pathological Q-waves, defined as new QS in 2 associated leads in the absence of LBBB, Wolf-Parkinson white syndrome; or new QS in 2 associated leads, defined as $\geq 0.04 \mathrm{~s}$ broad and/or $\mathrm{Q} / \mathrm{R}$ ratio $\geq 1 / 4$

Not defined

Not defined

Adjudicated by a CEC

Adjudicated by a CEC

Adjudicated by a CEC

Not defined

Adjudicated by a CEC
Not defined

Adjudicated by a CEC

Adjudicated by a CEC

Adjudicated by a CEC

Not defined

Adjudicated by a CEC 
Endpoint definitions

\begin{tabular}{ll}
\cline { 2 - 2 } Study & \multicolumn{1}{c}{ Cerebral stroke } \\
\hline Niranjan et al ${ }^{\mathrm{E} 65}$ & Adjudicated by a CEC \\
Nathoe et al [Octopus] ${ }^{\mathrm{E} 66}$ & Focal neurologic deficit, lasting $>24 \mathrm{~h}$, combined \\
& with an increase in disability of at least 1 grade on \\
& the Rankin Scale
\end{tabular}

Lemma et al [On-Off] ${ }^{\mathrm{E} 67}$

Onorati et $\mathrm{al}^{\mathrm{E} 68}$

Ozkara et al ${ }^{\mathrm{E} 69}$

Paparella et al ${ }^{\mathrm{E} 70}$

Parolari et al ${ }^{\mathrm{E} 71}$

Parolari et $\mathrm{al}^{\mathrm{E} 72}$

Penttilä et $\mathrm{al}^{\mathrm{E} 73}$

Straka $\mathrm{Z}$ et al [PRAGUE-4] ${ }^{\mathrm{E} 74}$

Hlavicka et al [PRAGUE-6] ${ }^{\mathrm{E} 75}$

Bednar et al [PRAGUE 11] [76 $^{\mathrm{E}}$

Sousa Uva et al [PROMISS] ${ }^{\mathrm{E} 77}$

Rachwalik et $\mathrm{al}^{\mathrm{E} 78}$

Rainio et $\mathrm{al}^{\mathrm{E} 79}$

Raja et al ${ }^{\mathrm{E} 80}$

Rasmussen et $\mathrm{al}^{\mathrm{E} 81}$

Rastan et al ${ }^{\mathrm{E} 82}$

Shroyer et al [ROOBY] $]^{\mathrm{E} 83}$

Sahlman et $\mathrm{al}^{\mathrm{E} 84}$

Sajja et $\mathrm{al}^{\mathrm{E} 85}$

Selvanayagam et al ${ }^{\mathrm{E} 86}$

Puskas et al [SMART] ${ }^{\mathrm{E} 87}$

Syed et $\mathrm{al}^{\mathrm{E} 88}$

Tang et $\mathrm{al}^{\mathrm{E} 89}$

Tatoulis et al ${ }^{\mathrm{E} 90}$

Tully et $\mathrm{al}^{\mathrm{E} 91}$
Not defined

Not defined

Not defined

Not defined

Not defined

Not defined

Global or focal neurologic deficit, lasting $<24 \mathrm{~h}$ or $>24 \mathrm{~h}$

Not defined

Not defined

Not defined

Not defined

Not defined

Not defined

Not defined

Not defined

Adjudicated by a CEC

Adjudicated by a CEC

Focal neurologic deficit lasting $>24 \mathrm{~h}$

Not defined

Focal neurologic deficit lasting $>24$ h, confirmed by

CT scan and neurologist examination

Not defined

Not defined

Defined according to Society of Thoracic Surgeons database definitions

Not defined
Myocardial infarction

Adjudicated by a CEC

Within $7 \mathrm{~d}$ after surgery: Non-Q-wave MI was diagnosed if the serum CK-MB isoenzyme level was $>5$ times the ULN value, and a Q-wave MI was diagnosed if pathological Q-waves appeared concomitantly.

At $7 \mathrm{~d}$ or more after surgery: non-Q-wave MI was diagnosed if the ratio of CK-MB isoenzyme to total CK exceeded 0.1, and Q-wave MI was diagnosed if pathological Q-waves appeared.

New Q-wave on the ECG with CK-MB isoenzyme fraction increase $>100 \mathrm{UI} / \mathrm{L}$ and/or $>10 \%$ of the total CK level and/or with documented new wall motion abnormalities other than septal on echocardiography.

Perioperative acute MI, defined by any of the following: new Q-waves $>0.04 \mathrm{~ms}$ with a peak $\mathrm{TnI}$ level $>3.7 \mu \mathrm{g} / \mathrm{L}$ or $\mathrm{TnI}$ concentration $>3.1 \mu \mathrm{g} / \mathrm{L}$ at $12 \mathrm{~h}$ following surgery; $>25 \%$ reduction in $\mathrm{R}$ waves in 2 or more ECG leads associated with the same troponin I peaks; or new akinetic or dyskinetic segments on echocardiography

Not defined

Not defined

Not defined

Not defined

Not defined

Q-wave MI, defined as ECG sign of necrosis, CK and CK-MB elevation, or new akinetic segment on echocardiography

Not defined

Not defined

Not defined

Not defined

Not defined

Not defined

Not defined

Not defined

Adjudicated by a CEC

CK-MB fraction release $>100 \mathrm{U}$ and new Q-wave

Not defined

Not defined

Not defined

Not defined

Not defined

Defined according to Society of Thoracic Surgeons database definitions

Two or more episodes of prolonged typical chest pain (>20 min) not relieved by rest and/or nitrates, enzyme level elevation, new wall motion abnormality, or new Q-wave in 2 leads on serial ECG 
APPENDIX TABLE 5. Continued

\begin{tabular}{|c|c|c|}
\hline \multirow[b]{2}{*}{ Study } & \multicolumn{2}{|c|}{ Endpoint definitions } \\
\hline & Cerebral stroke & Myocardial infarction \\
\hline Van Boven et $\mathrm{al}^{\mathrm{E} 92}$ & Adjudicated by a CEC & Adjudicated by a CEC \\
\hline Van Boven et $\mathrm{al}^{\mathrm{E} 93}$ & Adjudicated by a CEC & Adjudicated by a CEC \\
\hline Vedin et $\mathrm{al}^{\mathrm{E} 94}$ & Adjudicated by a CEC & Adjudicated by a CEC \\
\hline Velissaris et al ${ }^{\mathrm{E} 95}$ & Not defined & Not defined \\
\hline Velissaris et a ${ }^{\mathrm{E} 96}$ & Not defined & Not defined \\
\hline Vural et $\mathrm{al}^{\mathrm{E} 97}$ & Not defined & Not defined \\
\hline Wan et $\mathrm{al}^{\mathrm{E} 98}$ & Not defined & Not defined \\
\hline Wandschneider et $\mathrm{al}^{\mathrm{E} 99}$ & Not defined & Not defined \\
\hline Wehlin et $\mathrm{al}^{\mathrm{E} 100}$ & Not defined & Not defined \\
\hline Wehlin et al ${ }^{\mathrm{E} 101}$ & Not defined & Not defined \\
\hline Yu et $a^{\mathrm{E} 103}$ & $\begin{array}{l}\text { New acute focal neurologic deficit with signs and } \\
\text { symptoms lasting }>24 \mathrm{~h}\end{array}$ & $\begin{array}{l}\text { CK-MB release }>80 \mathrm{IU} / \mathrm{mL} \text { regardless of } \\
\text { concomitant changes on ECG or impaired } \\
\text { hemodynamics }\end{array}$ \\
\hline Zamvar et $\mathrm{al}^{\mathrm{E} 102}$ & Not defined & Not defined \\
\hline
\end{tabular}

$C T$, Computed tomography; $E C G$, echocardiography; $C K-M B$, creatine kinase muscle brain; $c T n I$, cardiac troponin I; $M I$, myocardial infarction; $B B S$, best bypass surgery trial; $T n T$, troponin T; BHACAS 1, beating heart against cardioplegic arrest study 1; CEC, Clinical Events Committee; BHACAS 2, eating Heart Against Cardioplegic Arrest Study 2; $L B B B$, left bundle branch block; TEE, transesophageal echocardiography; CORONARY, CABG off or on pump revascularization study; ST-T, ST-T wave; CRISP, The Coronary artery bypass grafting in high-RISk patients randomized to off- or on-Pump surgery; TnI, troponin I; ULN, upper limit of normal; DOORS, Danish on-pump versus off-pump randomization study; GOPCABE, German off-pump coronary artery bypass grafting in elderly patients; JOCRI, Japanese off-pump coronary revascularization investigation study; MRI, magnetic resonance imaging; MASS III, off-pump and on-pump stable multivessel coronary artery bypass grafting; QS, QS complex; Octopus, comparison of on-pump and off-pump CORONARY bypass surgery in low-risk patients; PRAGUE-4, A randomized comparison between off-pump and on-pump surgery PRAGUE-4; PRAGUE-6, Off-pump versus on-pump coronary artery bypass graft surgery in patients with EuroSCORE $\geq 6$; PRAGUE-11, A randomized comparison between off-pump and on-pump surgery PRAGUE-11; PROMISS, The prospective randomized comparison of off-pump and on-pump multivessel coronary artery bypass surgery; ROOBY, veterans affairs randomized on/off bypass study; SMART, surgical management of arterial revascularization therapies. 